\title{
Multi-Antenna and Relaying Techniques in Wireless Communication Networks
}

\author{
by Abdulkareem Bala Adinoyi, B.Eng, M.Sc.
}

A thesis submitted to

The Faculty of Graduate Studies and Research

in partial fulfillment of the requirements for the degree of

\author{
Doctor of Philosophy \\ Ottawa-Carleton Institute for Electrical and Computer Engineering \\ Department of Systems and Computer Engineering \\ Carleton University, Ottawa, Ontario \\ Canada
}

May, 2006

(C) Abdulkareem Adinoyi, 2006 


$\begin{array}{ll}\begin{array}{l}\text { Library and } \\ \text { Archives Canada }\end{array} & \begin{array}{l}\text { Bibliothèque et } \\ \text { Archives Canada }\end{array} \\ \begin{array}{l}\text { Published Heritage } \\ \text { Branch }\end{array} & \begin{array}{l}\text { Direction du } \\ \text { Patrimoine de l'édition }\end{array} \\ \begin{array}{l}\text { 395 Wellington Street } \\ \text { Ottawa ON K1A ON4 }\end{array} & \begin{array}{l}\text { 395, rue Wellington } \\ \text { Ottawa ON K1A ON4 } \\ \text { Canada }\end{array}\end{array}$

Your file Votre référence ISBN: 978-0-494-16659-8 Our file Notre référence ISBN: 978-0-494-16659-8

NOTICE:

The author has granted a nonexclusive license allowing Library and Archives Canada to reproduce, publish, archive, preserve, conserve, communicate to the public by telecommunication or on the Internet, loan, distribute and sell theses worldwide, for commercial or noncommercial purposes, in microform, paper, electronic and/or any other formats.

The author retains copyright ownership and moral rights in this thesis. Neither the thesis nor substantial extracts from it may be printed or otherwise reproduced without the author's permission.
AVIS:

L'auteur a accordé une licence non exclusive permettant à la Bibliothèque et Archives Canada de reproduire, publier, archiver, sauvegarder, conserver, transmettre au public par télécommunication ou par l'Internet, prêter, distribuer et vendre des thèses partout dans le monde, à des fins commerciales ou autres, sur support microforme, papier, électronique et/ou autres formats.

L'auteur conserve la propriété du droit d'auteur et des droits moraux qui protège cette thèse. $\mathrm{Ni}$ la thèse ni des extraits substantiels de celle-ci ne doivent être imprimés ou autrement reproduits sans son autorisation.
In compliance with the Canadian

Privacy Act some supporting forms may have been removed from this thesis.

While these forms may be included in the document page count, their removal does not represent any loss of content from the thesis.
Conformément à la loi canadienne sur la protection de la vie privée, quelques formulaires secondaires ont été enlevés de cette thèse.

Bien que ces formulaires aient inclus dans la pagination, il n'y aura aucun contenu manquant.

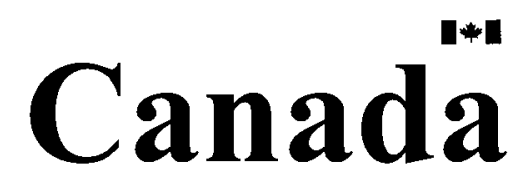




\section{Abstract}

This thesis investigates multi-antenna techniques within the context of distributed antenna and fixed relay networks. Such antenna and network architectures result in significant performance enhancements even in networks where the wireless terminals have only a single antenna.

Relaying, the use of intermediate nodes to help transmission from source to destination, has emerged as one paradigm shift in system deployment. Infrastructure-based relays are usually deployed by the service providers for coverage extension. The thesis investigates cooperative relaying to extend this paradigm even further. By exploiting the broadcast nature of wireless channels, fixed relay nodes are engaged in two-hop cooperative protocols as means for removing the burden of multiple antennas on wireless terminals, thus, providing end-toend (E2E) spatial diversity and network multiplexing benefits to terminals that are otherwise antenna-limited.

The deployment of a small number of antennas on infrastructure-based fixed relays is feasible, in contrast to mobile terminals; therefore, the thesis examines the impact of multiantenna on the distributed cooperative fixed relays. Maximal ratio combining and selection combining of these multiple antenna signals in threshold-based decode-and-forward relays are studied and analyzed. It is found that the multi-antenna multi-relay scheme could be used to improve the $\mathrm{E} 2 \mathrm{E}$ system performance, or for a given performance merit the multi-antenna component can significantly reduce the number of relays required in a service area. In addition, selection combining at the relays represents an excellent performance-cost tradeoff compared to single antenna relaying and maximal ratio combining based relaying.

Furthermore, relay-enabled user cooperation which exploits the infrastructure-based fixed relays is proposed. The explicit user cooperation diversity schemes studied in the literature require two willing users to form partnership. To sustain such a cooperative scheme, coercion or incentives for the cooperating partners might be needed, in addition to security concerns 
(as terminals have to detect partner's signals), which could present implementation challenges. Since the proposed cooperation schemes are transparent to the users, they present practical realizations for explicit user cooperation.

In all studied scenarios, simulations have been used to corroborate the system analyses conducted in the thesis. Mostly, the versatile Nakagami fading channel model has been used. 


\section{Acknowledgments}

I am particularly indebted to Dr. Halim Yanikomeroglu for his unconditional support, valuable guidance and endless patience he generously provided during the course of this work.

I am grateful to the examiners who served on my candidacy examination committee (Professors David Falconer, Yongyi Mao, and Florence Danilo-Lemoine) as well as to the examiners who sat on my thesis examination committee (Professors Elvino Sousa, Abbas Yongacoglu, Florence Danilo-Lemoine, Ian Marsland, and Len MacEachern) for their time and efforts. Their assistance can never be appreciated enough.

The work was supported in part by the Natural Sciences \& Engineering Research Council of Canada under participation in the Wireless world Initiative New Radio (WINNER) project. I thankfully acknowledge this support.

To my wife Khadeejah and our children - Nimatullah-Ize, Yusra-Ozohu, Sakeena-Ozavize and Saleema-Onize - goes my deepest gratitude for their patience and support through this period. The smiles and 'chaos' these children occasionally create at home remove the boredom and monotonous office routines, after all, chaos begets entropy. Finally, may God Almighty, "the uncaused cause of all being (as-Samad)", show mercy to my dad - Suleiman Adinoyi who left this world few months before this finish line. 


\section{Contents}

$\begin{array}{lll}\text { Abstract } & \text { iii }\end{array}$

$\begin{array}{ll}\text { Acknowledgments } & \text { v }\end{array}$

List of Abbreviations $\quad$ xvi

List of Symbols $\quad$ xix

1 Introduction 1

1.1 Research Motivation . . . . . . . . . . . . . . . . 1

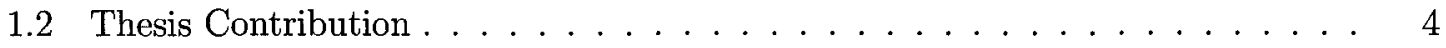

1.3 Thesis Organization . . . . . . . . . . . . . . 7

2 Wireless Communication Systems: Challenges and Techniques 9

2.1 Multiple Antennas and Spatial Diversity . . . . . . . . . . . 9

2.1 .1 Macrodiversity . . . . . . . . . . . . . . . 12

2.1 .2 Microdiversity . . . . . . . . . . . . . . . 13

2.2 Relaying and the Notion of Cooperative Diversity . . . . . . . . . . . 17

2.2.1 Relaying and Multihop Techniques . . . . . . . . . . . . . 17

2.2 .2 Relaying Classification . . . . . . . . . . . . . . . . . . 19

2.2 .3 Virtual Antenna Arrays . . . . . . . . . . . . . . . . . 21

2.2 .4 Cooperative Networks $\ldots \ldots \ldots \ldots$. . . . . . . . . . . 21

vi 
2.3 Models for Fading Channels . . . . . . . . . . . . . . . . 25

2.3 .1 Fading Models . . . . . . . . . . . . . . 26

2.3.2 Decode-and-Forward Probability in Multi-antenna Systems . . . . . . . 31

2.4 Chapter Conclusion . . . . . . . . . . . . . . . . 36

3 Hybrid Macro- and Micro-diversity Antenna Networks 37

3.1 System and Channel Descriptions . . . . . . . . . . . . . . . . . . . . 39

3.2 Macrodiversity Selection Schemes . . . . . . . . . . . . . . . 40

3.2 .1 Scheme I . . . . . . . . . . . . . . . . 40

3.2 .2 Scheme II . . . . . . . . . . . . . . . . . . . 41

3.2 .3 Scheme III . . . . . . . . . . . . . . . . . . . . 42

3.3 Performance Analysis . . . . . . . . . . . . . . . . . . . . . . 42

3.3.1 Numerical Results . . . . . . . . . . . . . . . . . 44

3.4 Outage Probability Calculation . . . . . . . . . . . . . . 48

3.5 Effects of Correlation on System Performance . . . . . . . . . . . . . . . . 52

3.6 Notes on Implementation and System Complexity . . . . . . . . . . . 57

3.7 Chapter Conclusion . . . . . . . . . . . . . . . . . . . . . 59

4 Multi-antenna Cooperative Relay Networks $\quad 61$

4.1 System of Multi-antenna Multi-relay . . . . . . . . . . . . . 63

4.2 Formulation of the E2E System Error Performance . . . . . . . . . . . . 64

4.2 .1 Error Rate Analysis . . . . . . . . . . . . . . . . . . . 65

4.2.2 Relay Error Performance Analysis . . . . . . . . . . . . . . 68

4.2.3 MRC-based Multi-antenna Relay and Threshold Decode-and-Forward Strategy ............................ 70

4.2.4 SC-based Multi-antenna Relay and Threshold Decode-and-Forward Strategy ............................ 73

vii 
4.3 Threshold Decode-and-Forward Protocol vs Multiple Antennas: Which Way? . 81

4.4 Numerical Performance Illustration of Multi-antenna Multi-relay . . . . . . . . 82

4.5 On the System Complexity and Bandwidth Preservation . . . . . . . . . 95

4.6 Chapter Conclusion . . . . . . . . . . . . . . . . . 97

5 Asymmetric Relay Networks $\quad 98$

5.1 Analysis . . . . . . . . . . . . . . . . . . . . . 100

5.2 Scenarios. . . . . . . . . . . . . . . . . . . . . 102

5.3 Numerical Illustration $\ldots \ldots \ldots \ldots$

5.4 Chapter Conclusion . . . . . . . . . . . . . . . . . 110

6 Fixed Relay-enabled User Cooperation $\quad 111$

6.1 Motivation ................................. 111

6.2 System Descriptions . . . . . . . . . . . . . . . . 113

6.3 Analysis of Relay-enabled Cooperative Communication Schemes . . . . . . . 116

6.4 Error Rate for Transmit Diversity . . . . . . . . . . . . . . . . . . 122

6.4.1 Numerical Examples: Performance of Relay with SDMA (Optimum Combining $\ldots \ldots \ldots \ldots \ldots \ldots \ldots$. . . . . . . . . . . . . . . . . . . . .

6.4.2 E2E Network Performance: Simulation Examples with Infinite Power Interferer . . . . . . . . . . . . . . . . . . 125

6.4.3 E2E Network Performance: Analytical Examples for Different Power Levels of Partner . . . . . . . . . . . . . . . . . . 129

6.5 Chapter Conclusion . . . . . . . . . . . . . . . . . . . . 131

7 Conclusions and Recommendation $\quad 135$

7.1 Recommendation for Future Research . . . . . . . . . . . . . . . . 138

$\begin{array}{lr}\text { A Published and Submitted Work } & 140\end{array}$

viii 
B Practical Capacity Calculation for Time-Hopping Ultra-wide Band Multiple$\begin{array}{ll}\text { Access Communications } & 143\end{array}$

B.1 Introduction . . . . . . . . . . . . . . . . . . 143

B.2 Random Coding Error Exponent . . . . . . . . . . . . . . . . . . 144

B.3 Numerical Results . . . . . . . . . . . . . . . . . . . . . . . . . 148

B.3.1 Coding Complexity Measure . . . . . . . . . . . . . . . 150

B.4 Conclusion . . . . . . . . . . . . . . . . . . . 151

$\begin{array}{ll}\text { C Derivations } & 152\end{array}$

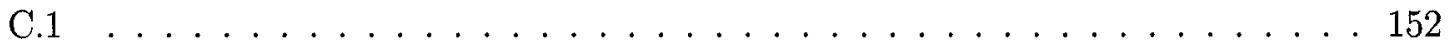

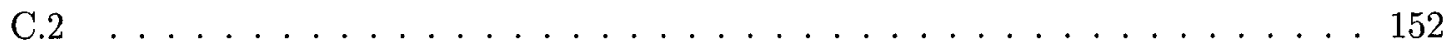

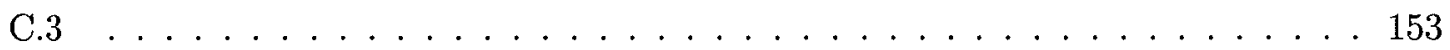

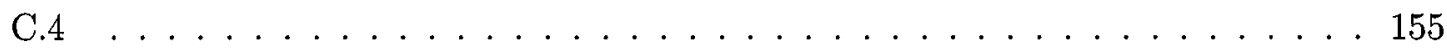

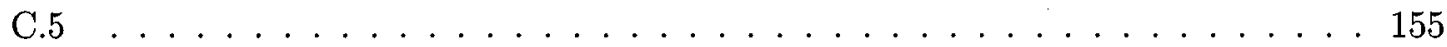




\section{List of Figures}

2.1 MIMO channels. . . . . . . . . . . . . . . . . . . . 10

2.2 Capacity of a channel with $\left(a_{t}, a_{r}\right)$ MIMO for various value of SNR and $a_{t}=a_{r} .12$

2.3 Performance of binary signals with MRC in Rayleigh fading channels. . . . . . 15

2.4 Conventional relay networks, (a) conventional single-hop network, (b) conventional two-hop relay network, (c) combatting shadowing with a relay. . . . . . 18

2.5 User cooperation. . . . . . . . . . . . . . . . . . . 22

2.6 User cooperation and resource sharing (a) direct, multiple access transmission (MAI), terminals $T_{1}$ and $T_{2}$ transmit at the same time, (b) orthogonal direct transmission, (c) orthogonal cooperative diversity. . . . . . . . . . . . . 24

2.7 Network configuration for parallel relays: (a) single cooperating terminal, (b) two cooperating terminals. . . . . . . . . . . . . . . . 24

2.8 The CDF of Nakagami distribution with different values of $m \ldots \ldots$. . . . 29

2.9 Probability of decoding in threshold-based decode-and-forward protocol. . . . 30

2.10 The probability of decode of SC-based threshold decode-and-forward relay with different number of antennas $(L)$ for $m=1,2,4,6$, average SNR $=10 \mathrm{~dB} \ldots . \quad 34$

2.11 Probability of decode in MRC-based threshold-based decode-and-forward protocol, average $\mathrm{SNR}=10 \mathrm{~dB} \ldots \ldots \ldots \ldots \ldots$

3.1 Antenna layout for the macro/microdiversity in a wireless network. . . . . . . 39 
3.2 Performance of BPSK in conventional macrodiversity and GSC-based microdiversity (Scheme I) with lognormal shadowing $(\sigma=12 \mathrm{~dB})$ and Rayleigh fading; $K=3, L=5,1 \leq l_{\mathrm{o}} \leq L \ldots \ldots \ldots \ldots \ldots \ldots \ldots$

3.3 Performance of BPSK in conventional macrodiversity and GSC-based microdiversity (Scheme I) with lognormal shadowing $(\sigma=8 \mathrm{~dB})$ and Rayleigh fading; $K=2, L=5,1 \leq l_{\mathrm{o}} \leq L \ldots \ldots \ldots \ldots \ldots \ldots \ldots$

3.4 Performance of 8-PSK in conventional macrodiversity and GSC-based microdiversity (Scheme I) with lognormal shadowing $(\sigma=12 \mathrm{~dB})$ and Rayleigh fading.

3.5 Performance comparison of the selection schemes with $\sigma=8 \mathrm{~dB}$ for different network configurations for BPSK. . . . . . . . . . . . . . . 49

3.6 Outage probability of QPSK with Scheme I $(\sigma=6 \mathrm{~dB}) \ldots \ldots \ldots \ldots$

3.7 Performance of BPSK with Scheme I in correlated macrodiversity with $\sigma=12$ $\mathrm{dB} ; K=2, L=5, l_{\circ}=2, \rho=0,0.2,0.5,0.7,1.0 \ldots \ldots \ldots \ldots \ldots$

3.8 Performance of BPSK with Scheme I in correlated macrodiversity with $\sigma=12$ $\mathrm{dB} ; K=2, L=5, l_{\mathrm{o}}=4, \rho=0,0.2,0.5,0.7,1.0 \ldots \ldots \ldots \ldots \ldots \ldots$

3.9 Effect of correlated shadowing on the performance of BPSK with Scheme II for two different $\sigma$ values, and $K=2, L=5, l_{\circ}=3 \ldots \ldots \ldots \ldots$

3.10 Effect of correlated shadowing on the performance of BPSK with Scheme III for two different $\sigma$ values; $K=2, L=5, l_{\circ}=3 \ldots \ldots \ldots \ldots 6$

4.1 Relay networks (a) conventional fixed decode and forward (b) cooperative relaying, (c) multi-antenna cooperative relay terminals. . . . . . . . . . . . 63

4.2 BER of relay implementing threshold decoding in single channel fading, $m=1$. 71

4.3 BER of MRC-based TDF relay in fading channel, $m=1$ : Simulation and Analysis. . . . . . . . . . . . . . . . . 76

4.4 BER of MRC-based TDF relay in Nakagami fading, $m=2,6 \ldots \ldots \ldots 77$ 
4.5 Error performance comparison of MRC-based vs SC-based TDF relay in fading channel, $m=1$ : MRC solid curves, SC the broken curves. . . . . . . . 78

4.6 Error performance comparison of MRC-based vs SC-based TDF relay in fading channel, $m=2:$ MRC solid curves, $\mathrm{SC}$ the broken curves. . . . . . . . . . 79

4.7 Analytical and simulated error performance of SC-based TDF relay in Nakagami fading $(m=1,2)$ and $L=1,2,5$ : solid curves represent the analysis, the symbols relate to the simulations. . . . . . . . . . . . . . . . 80

4.8 E2E BER as a function of a relay decoding threshold $\left(N_{R}=1, L=1\right) . \quad \ldots \quad 83$

4.9 E2E BER as a function of relay decoding threshold $\left(N_{R}=2\right.$, and $\left.L=1,2\right)$. . 84

4.10 E2E BER as a function of a relay decoding threshold $\left(N_{R}=1,2\right.$, and $\left.L=2,4\right) . \quad 85$

4.11 E2E BER comparison for one cooperating relay, threshold vs multiple antennas. 86

4.12 The E2E BER of single antenna $(L=1)$ parallel relays with threshold decoding in Nakagami fading: Analysis (solid curves) and Simulation (symbols). . . . . 87

4.13 BER performance of MRC-based multi-antenna TDF relay in Nakagami fading, $m=1,2,6$ and $N_{R}=1 \ldots \ldots \ldots \ldots \ldots \ldots \ldots$

4.14 BER performance of MRC-based multi-antenna TDF relay in Nakagami fading,

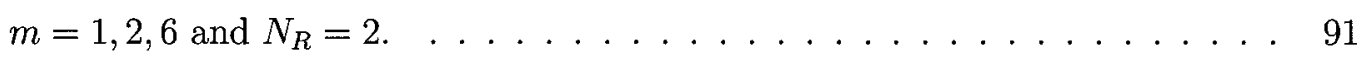

4.15 BER performance of MRC-based multi-antenna TDF relay in Nakagami fading, $m=1,2,6$ and $N_{R}=4 \ldots \ldots \ldots \ldots \ldots$

4.16 Performance comparison of MRC-based vs SC-based multi-antenna TDF relay in Nakagami fading, $m=1,2,6 \ldots \ldots \ldots \ldots$

5.1 Two-hop network configurations (a) conventional relaying, (b) cooperative network, (c) cooperative network (parallel relays) . . . . . . . . . . . . . 99

5.2 Cooperative error performance at the destination of two-hop relay networks. 105

5.3 Comparison of the bound and simulated end-to-end error performance of relay networks in Rayleigh fading. . . . . . . . . . . . . . . . . . . 107

xii 
5.4 Comparison of the bound and simulated end-to-end error performance of relay networks in Rayleigh fading. . . . . . . . . . . . . . . . . . . . . 108

5.5 End-to-end BER performance of relay networks with different relay locations and $m$-parameter. . . . . . . . . . . . . . . . . . . . 109

6.1 Fixed relay-enabled user cooperation: Realization I. . . . . . . . . . . . . . 114

6.2 Fixed relay-enabled user cooperation: Realization II. . . . . . . . . . . . . . 114

6.3 Fixed relay-enabled user cooperation: Realization III. . . . . . . . . . . . . . 115

6.4 Fixed relay node equipped with multi-antenna. The relay uses spatial division multiple access technique to detect a desired user while directing a null to an interfering user $($ realization $\mathrm{I}) \ldots \ldots \ldots \ldots$. . . . . . . . . . . 117

6.5 Performance of a relay performing optimum combining in Rayleigh fading channels. The BER of the desired user is shown for different levels of power of partner $(L=1, L=2) \ldots \ldots \ldots \ldots \ldots \ldots \ldots$

6.6 Performance of a relay performing optimum combining in Rayleigh fading channels. The BER of the desired user is shown for different levels of power of

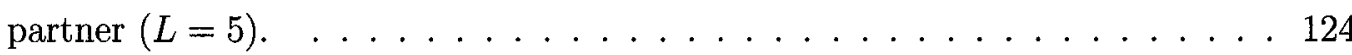

6.7 Performance of a relay performing optimum combining in Rayleigh fading channels for the desired user for different power levels of partner $L=2,8$-PSK. . . 125

6.8 Performance of a relay performing optimum combining in Rayleigh fading channels for the desired user for different power levels of partner ( $L=3$ and $L=5)$,

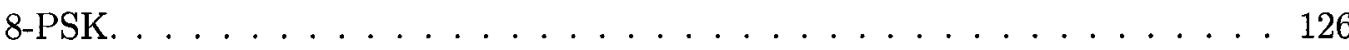

6.9 E2E performance of relay-enabled user cooperation in Rayleigh fading channels with one receive antenna at the destination (virtual $2 \times 1$ antennas in the second hop). .......................... 127

xiii 
6.10 E2E performance of relay-enabled user cooperation in Rayleigh fading channels with two receive antennas at the destination (virtual $2 \times 2$ antennas in the second hop). . . . . . . . . . . . . . . . . . 128

6.11 The E2E SER of the desired user for different power levels of partner $(L=2$, 8-PSK)

6.12 The E2E SER of the desired user for different power levels of $\operatorname{partner}(L=3$, 8-PSK).

6.13 The E2E simulated SER of the desired user for infinite power of partner $\left(\mathrm{SNR}_{2}=\right.$ $\infty \mathrm{dB})$ and the analytical SER for $\mathrm{SNR}_{2}=40 \mathrm{~dB}$ (analysis) $(L=2,3,5,8$-PSK). 133

B.1 Cutoff rates of M-ary PPM multiuser UWB for two different spreading factors at $\mathrm{SNR}=20 \mathrm{~dB}$ in the absence of time hopping $\left(N_{s}=1\right) \ldots \ldots \ldots$

B.2 Cutoff rates of M-ary PPM multiuser UWB for two different spreading factors for $\mathcal{N}_{u}=100$ in the absence of time hopping $\left(N_{s}=1\right) \ldots \ldots \ldots$

B.3 Cutoff rates of TH 2-PPM multiuser UWB for different spreading and hopping factors for $\mathcal{N}_{u}=100$.

xiv 


\section{List of Tables}

4.1 MRC-based and SC-based Relay Detection: System SNR Comparison at BER $=10^{-4} \ldots \ldots \ldots \ldots \ldots \ldots \ldots \ldots \ldots \ldots \ldots \ldots \ldots \ldots \ldots \ldots \ldots \ldots$

6.1 The encoding and transmission sequence for the distributed space time code . 116

B.1 Code lengths for PPM signaling at rate $2.0 \mathrm{bits} / \mathrm{symbol} \ldots \ldots \ldots \ldots$. . . . . 150 


\section{List of Abbreviations}

$4 \mathrm{G}$

AdDF

$\mathrm{AF}$

$\mathrm{AmF}$

AWGN

BER

BLAST

BPSK

BS

CDMA

CDF

$\mathrm{CF}$

CRC

$\mathrm{CU}$

DA

DBLAST

DF

DFP

DSTC

$\mathrm{E} 2 \mathrm{E}$

EGC

FD

FDD

FRN

GSC
Fourth Generation

Adaptive Decode-and-forward

Amplify-and-Forward

Amount of Fading

Additive White Gaussian Noise

Bit Error Rate

Bell Laboratory Layered Space-Time architecture

Binary Phase Shift Keying

Base Station

Code Division Multiple Access

Cumulative Density Function

Characteristic Function

Cyclic Redundancy Check

Central Unit

Distributed Antennas

Diagonal Bell Laboratory Layered Space-Time architecture

Decode-and-Forward

Decode-and-Forward Probability

Distributed Space Time Coding

End-to-End

Equal Gain Combining

Full Duplex

Frequency Division Duplexing

Fixed Relay Node

Generalized Selection Combining

xvi 


\begin{tabular}{|c|c|}
\hline HiperLAN & High performance Radio Local Area Networks \\
\hline HRC & Hurwitz-Radon Code \\
\hline LOS & Line-of-Sight \\
\hline MAS & Multi-Antenna Systems \\
\hline MAI & Multiple Access Interference \\
\hline MBWA & Mobile Broadband Wireless Access \\
\hline MDM & Multiply Detected Macrodiversity \\
\hline MIMO & Multiple Input Multiple Output \\
\hline MPSK & M-ary Phase Shift Keying \\
\hline $\mathrm{MRC}$ & Maximal Ratio Combining \\
\hline MQAM & M-ary Quadrature Amplitude Modulation \\
\hline MUD & Multi-User Detection \\
\hline NLOS & Non-Line-of-Sight \\
\hline PDF & Probability Density Function \\
\hline RoF & Radio-over-Fiber \\
\hline $\mathrm{R}-\mathrm{D}$ & Relay to Destination Link \\
\hline $\mathrm{RF}$ & Radio Frequency \\
\hline $\mathrm{RV}$ & Random Variable \\
\hline $\mathrm{SC}$ & Selection Combining \\
\hline S-D & Source to Destination Link \\
\hline SDA & Sectorized Distributed Antennas \\
\hline SDMA & Spatial Division Multiple Access \\
\hline SER & Symbol Error Rate \\
\hline SNR & Signal-to-Noise Ratio \\
\hline S-R & Source to Relay Link \\
\hline SRake & Selective Rake \\
\hline
\end{tabular}

xvii 
Turbo Code

TDD

Time Division Duplexing

TDF

Threshold Decode-and-Forward

TH-PPM

Time Hopping Pulse Position Modulation

UWB

Ultra-wide Band

VAA

Virtual Antenna Array

WiMax

Worldwide Inter-operability Microwave Access

$\mathrm{WiFi}$

Wireless Fidelity

WINNER

Wireless World Initiative New Radio

WLAN

Wireless Local Area Networks

WPAN

Wireless Personal Area Networks

WMAN

Wireless Metropolitan Area Networks

xviii 


\section{List of Symbols}

\begin{tabular}{|c|c|}
\hline$L$ & Number of receive (diversity) branches \\
\hline$l_{\text {。 }}$ & Order of generalized selection combining \\
\hline$m$ & Nakagami fading parameter \\
\hline$h, q$ & MPSK constellation parameters \\
\hline$\alpha, \alpha$ & Fading sample, vector \\
\hline$\mu_{k}$ & Shadowing common mean \\
\hline$\Lambda_{k}$ & Lognormal shadowing sample \\
\hline$g, g^{*}$ & Gaussian samples for the realization of $\Lambda$ \\
\hline$\sigma_{k}, \sigma$ & Shadowing standard deviation \\
\hline$\rho$ & Correlation factor \\
\hline$\gamma$ & Instantaneous SNR \\
\hline $\bar{\gamma}$ & Average SNR (usually per branch) \\
\hline$\Omega$ & Channel mean power \\
\hline$\Gamma[\cdot]$ & Gamma function \\
\hline$\Gamma[\cdot, \cdot]$ & Incomplete gamma function: $\Gamma[a, b]=\int_{b}^{\infty} t^{a-1} e^{-t} d t$ \\
\hline$\Gamma_{\mathrm{L}}[\cdot, \cdot]$ & Lower incomplete Gamma function: $\Gamma_{\mathrm{L}}[a, b]=\int_{0}^{b} t^{a-1} e^{-t} d t$ \\
\hline$B_{x}[\cdot, \cdot]$ & Incomplete beta function \\
\hline${ }_{1} F_{1}[\cdot, \cdot, \cdot]$ & Confluent hypergeometric function \\
\hline${ }_{2} F_{1}[\cdot, \cdot ; \cdot ; \cdot]$ & Hypergeometric function \\
\hline$\Omega_{\mathrm{sr}}$ & Source-relay channel power \\
\hline$M$ & Cardinality of modulation scheme \\
\hline $\mathcal{K}$ & Ricean factor \\
\hline$K$ & Number of macro-diversity ports \\
\hline$\kappa$ & Path loss exponent \\
\hline$a_{t}, a_{r}$ & Number of MIMO transmit antennas, receive antennas \\
\hline
\end{tabular}

xix 
$\mathcal{R}$

$\mathcal{R}_{0}$

$\nabla_{g}$

$\operatorname{div}\left(\nabla_{g}\right)$

$\mathbf{H}$

$x, \mathbf{x}$

y

n

$(x, y)$

$v$

$\phi_{i}$

$\mathcal{N}_{u}$

W

$r$

$P L$

$S_{\text {loss }}$

$T_{i}$

$G_{a}$

$N, N_{1}, N_{2}$

$P_{b}$

$P_{e}$

$\mathcal{P}_{r}$

$\mathcal{P}_{t}$

$\varpi_{p}, \varpi_{a}$

$c_{j}^{(k)}$

$\mathcal{D}_{j}^{(k)}$
Data rate

Cutoff rate

MIMO multiplexing gain

Diversity order at $\nabla_{g}$ in MIMO system

MIMO channel matrix

Transmitted signal, signal vector

Received signal vector

AWGN

Cartesian coordinate

Eigenvector

An eigenvalue

Number of users

Bandwidth

Distance between two communicating nodes, e.g., source and destination

Path loss

System loss

Terminal $i$

Antenna gain

Code or frame length

Probability of bit error

Probability of symbol error

Received power

Transmit power

UWB slot signal strength when signal is present, absent

UWB hopping code

PPM time shift 
$\mathcal{T}_{f}$

$\mathcal{T}_{p}$

$\mathcal{T}_{s}$

$\psi_{i}$

$N_{s}$

$\mathrm{R}, \mathbf{R}$

UWB frame time

Monocyle duration

Symbol duration

Template function

Number of UWB hops

Correlation function, matrix

xxi 


\section{Chapter 1}

\section{Introduction}

\subsection{Research Motivation}

The conventional cellular architecture appears incapable of delivering the ubiquitous high data-rate coverage expected of the future generation of wireless systems. The intended coverage, quality of service and transmission rates of these systems are order of magnitude higher than that supported by the present generation systems. Therefore, there are excessive expectations put on certain communication resources such as scarce radio spectrum and the link budget. Even the recent advances in antenna technologies (such as smart antennas and MIMO systems) and signal processing techniques (such as advanced channel coding methods) alone do not seem sufficient to alleviate the potential stress that is caused to the link budget [1]. The inadequacy of the conventional cellular architecture requires a fundamental change in the way systems are designed and deployed as well as novel signal processing techniques. One of the promising strategies is the incorporation of multihop capability in the current wireless networks. This is believed to be the most feasible architectural upgrade towards delivering almost ubiquitous high data rate coverage. Due to the cost-effectiveness of this approach, interest in the concept of relaying has developed in networks such as next generation cellular (4G) systems: WLAN (WiFi/HiperLAN2), and broadband fixed wireless (802.16/WiMax) 
networks. For example, IEEE 802.16 WiMax standard has provisions for creating a multi-hop mesh [2].

In the IEEE 802 wireless world framework, a number of working groups are focused on developing mesh-enabled standards IEEE 802.11s - WLAN (Wireless Local Area Network), IEEE 802.15.5 - WPAN (Wireless Personal Area Network) Mesh Networking, IEEE 802.16 WMAN (Wireless Metropolitan Area Network), IEEE 802.16-2004 standard entitled "air interface for Fixed Broadband Wireless Access Systems", whose MAC layer supports a primarily point-to-multi-point architecture, with an optional mesh topology and IEEE 802.20 - MBWA (Mobile Broadband Wireless Access). In addition to these on-going standardization efforts, various proprietary mesh/relay network solutions in the unlicensed bands are also being developed by various industrial players. The emergence of the relay-enabled standards in the IEEE 802 family predicts much higher interest and activity for relay-based communications. Already, the WINNER project ${ }^{1}$ is developing relay-enabled deployment concept for ubiquitous broadband mobile radio access network. These relay deployment technologies are expected to integrate wide area and short range scenarios thus closing the gap between WLAN-type and cellular systems [3].

Furthermore, since the low and favorable bands are already highly utilized, ${ }^{2}$ the future systems will be operating at frequency bands well above those of the present systems. For instance, the BWA (IEEE 802.16) as approved in 2001, addresses frequencies from 10 to $66 \mathrm{GHz}$ [4]. In these high frequency bands, the radio propagation is more susceptible to propagation conditions and distance dependent attenuation thereby introducing significant deployment challenges. In addition, wireless signals experience multipath fading, which is a result of the unguided nature of wireless channels with the consequence of significant burden

\footnotetext{
${ }^{1}$ WINNER - Wireless world Initiative New Radio, https://www.ist-winner.org/

${ }^{2}$ Today, a host of new technologies (mobile phones, radio and TV broadcasting, satellites, even entertainment services) are vying with existing radio-based applications for a slice of the valuable, but crowded, radio spectrum [5].
} 
on the link budget.

An uninformed approach to obtaining ubiquitous service could be increasing significantly the density of base stations in a given area resulting in higher deployment costs. This is not a feasible option for a number of reasons. First, with the current level of penetration of wireless systems, the subscriber growth is much smaller than the value of such network. Second, the fewer the base stations per unit area, the higher the economic value for the network provider is.

Therefore, it is necessary to establish efficient and cost-effective strategies for deploying system resources to minimize the cost-per-bit for both the service provider and end users. In this relation, Metcalfe's socio-economic law ${ }^{3}$ should be recognized, it puts an inordinate burden on the initiation of a network service that requires a significant capital cost to both the network provider and consumer.

The first part of the thesis is the research conducted prior to the participation in the WINNER project. This portion is divided into two parts - distributed antenna systems and ultra-wide band (UWB) systems. The work on 'dumb' distributed antenna systems presented in Chapter 3 lays the foundation for the concept of 'intelligent' distributed relaying. Unrelated to the core of this thesis is the research on UWB, a field that is attracting increasing interest. The initial results obtained from this research are presented in Appendix B.

The second part of the thesis proposes and analyses a number of system deployment concepts. The proposed systems combine the use of multi-antenna and fixed relaying techniques that are suitable for deploying future wireless systems. This part of the thesis - Chapters 2, 4, 5 , and 6 - represents a portion of Carleton University's contribution to the WINNER project ${ }^{4}$. WINNER is a consortium of about 40 partners, with center of gravity in Europe, working

\footnotetext{
${ }^{3}$ Metcalfe's law states that the value of any communication network grows as the square of the number of users of the network [6].

${ }^{4}$ The work was supported in part by the Natural Sciences \& Engineering Research Council of Canada under participation in the WINNER project.
} 
towards enhancing the performance of wireless communication systems. The improvements of radio transmission to be explored by WINNER are crucial for enabling user-eccentric services, for any application, anytime and anywhere.

\subsection{Thesis Contribution}

In the context of distributed antenna systems

- The thesis proposes a microdiversity-augmented macrodiversity architecture to combat two fading phenomena (small-scale fading and shadowing) in one scheme. A $K$-macro $/ L$ microdiversity antenna structure is proposed with the following algorithm - the macrodiversity selects the best among $K$ ports, using certain criteria, and of the $L$ signals available at that port, $l_{\circ}$ strongest are selected for diversity combining. Generalized selection combining (GSC) is employed as the receiver processor for the microdiversity component since it represents a reasonable system complexity and cost compromise compared to a full blown maximal ratio combining (MRC).

- With microcellular networks in mind, a different approach to the conventional cellular concept which eliminates the need for expensive base station resources is proposed. The thesis considers a wireless network with cost-efficient radio access ports which are linked to a central unit $(\mathrm{CU})$. The thesis then:

- Introduces some novel means of implementing the macro-selection (Scheme II), which is superior to the conventional method.

- Explores macrodiversity MRC in microcellular systems referred to as Scheme III, which can be viewed as a natural extension of the CDMA cellular soft handover.

- Provides the outage performance and error rate analyses for the architectures that are proposed. 
In the context of multi-antenna cooperative relaying

- The thesis investigates the use of infrastructure-based fixed relays for providing spatial diversity gains for a wireless terminal which, otherwise, has limitations in the number of antennas it can bear. In the contemporary context, relays are used for coverage extension. In addition to the coverage extension, and by exploiting the broadcast nature of wireless channels, the fixed relay nodes are engaged in two-hop cooperative protocols with the source to provide E2E spatial diversity and network multiplexing benefits to wireless terminals. Thus, the burden of multiple antennas on these terminals is removed.

- Service provider deployed fixed relays have the potential to carry multiple antennas. The thesis, therefore, studies the impact of multi-antenna on cooperative two-hop networks. The single antenna relaying is a special case of this set-up. In addition, threshold maximal ratio combining and threshold selection combining of the multi-relay multiantenna schemes are analyzed. Threshold decoding, crucial to single antenna relays, is a measure towards combating error propagation. This error propagation affects the system E2E performance in an adverse manner; reliable decoding of the source-relay channel is essential for any cooperative scheme.

- Based on the analytical formulation and results, a number of observations, which can be useful in network design and deployment, could be made. Here are two such important observations. First, the E2E error performance of a network which has few relays with multiple antennas is not significantly worse than that which has many relays with single antenna. Obviously, the former network has a tremendous deployment cost advantage over the latter. This situation is due to the following fact: In a network with many relays, there is a potential for obtaining a high diversity order. However, when each relay has only single antenna, at any given time only a fraction of these relays actually do retransmit the received signal (due to the SNR threshold-based relaying decision to prevent error propagation). As a result, the mentioned potential of high diversity order 
is not fully achieved. On the other hand, when there are multiple antennas at each relay, reliable forwarding almost always occurs. Therefore, the full potential of each relay is achieved, and due to the saturation of the diversity benefits, a few relays will be sufficient to combat against fading. Second, the E2E error performance of a network in which the multiple antennas at relays are configured in maximal ratio combining (MRC) fashion is not significantly better than the performance of a network in which selection combining $(\mathrm{SC})$ is used. For implementation, $\mathrm{SC}$ requires one radio frequency (RF) detection chain as compared to a full-blown MRC that requires as many RF detection chains as the number of antennas.

- The commonly assumed symmetry in multiple relay channels is unrealistic. This assumption, though convenient for analysis, is not applicable to many practical systems. Building on the cooperative relaying described earlier, the performance of relay deployment where the cooperating nodes could be at any arbitrary positions is analyzed. Such set-ups are referred to as asymmetric networks. Furthermore, this treatment assumes that these relays could experience different channel distributions to the destination. For example, some relay-destination links can be modelled as a non-line of sight (Rayleigh fading) while others as a line of sight (Ricean fading). Therefore, it is easy to investigate different network topologies.

- The thesis also proposes relay-enabled user cooperation. The relay-enabled user cooperative diversity scheme is proposed to avoid the problems of user-dependent or explicit user cooperation diversity schemes. In this cooperation strategy, two users, ignorant of their cooperation, are engaged in it through two fixed relays. There are many benefits of this approach. First, the privacy of the users is not compromised since partners do not detect each other's data as it is the case with explicit user cooperation. Second, sanctions or rewards to facilitate user cooperation are not required. Third, network quality and services are not specifically user-dependent. Fourth, the proposed scheme, does 
not require any modifications on current terminals. In contrast, terminal modification is required for the terminals to support the explicit user cooperation. Therefore, there is a potential of using the proposed cooperation schemes within some existing wireless networks. Finally, the new scheme provides bit error rate and network spectral efficiency that are superior to the referenced conventional transmissions.

- After demonstrating the potential gains of the proposed architectures and algorithms as compared to the reference schemes, we then draw attention whenever it is applicable to some practical, complexity and implementation issues, which need to be considered towards realizing these gains.

- Finally, prior to the research leading to the contributions listed above, the cutoff rate of time-hopping (TH) ultra-wide band (UWB) communication system is evaluated for multiple-access channels. The cutoff rate can be used for determining various system trade-offs. For instance, it is shown that if synchronization problems would preclude high spreading factors, a suitable number of hops can be used instead to achieve the same performance. Moreover, it is demonstrated that the cutoff rate can be a fast way of gaining insights into the multiuser capacity of TH-PPM UWB systems.

\subsection{Thesis Organization}

The rest of the thesis is organized as follows. Chapter 2 presents an overview of the challenges faced by wireless communications. It also discusses relaying and wireless channel models. In Chapter 3, the proposed $K$-macro/ $L$-microdiversity antenna architecture is discussed and the various selection techniques proposed for this architecture are discussed. In Chapter 4 the proposed multi-antenna multi-relay techniques are presented and analyzed. Chapter 5 extends the proposed scheme of Chapter 4 to asymmetric channels while Chapter 6 discusses the relay-enabled cooperative communication schemes. Chapter 7 gives the main conclusions 
on the research in multi-antenna and cooperative relaying schemes. In addition, some recommendations for future research work have been outlined. Finally, Appendix B represents the work conducted on ultra-wide band techniques which was published in the July 2005 issue of the IEEE Communication Letters. 


\section{Chapter 2}

\section{Wireless Communication Systems: Challenges and Techniques}

\subsection{Multiple Antennas and Spatial Diversity}

High data rates and quality of service are the demands of the explosive market of the future wireless communication. The multiple input multiple output (MIMO) technique is an emerging technology that dramatically increases the spectral efficiency and capacity of wireless systems. This technique uses arrays of antenna elements at both the transmitting and receiving ends [7]. These antennas can be used to provide diversity gains for a given data rate $(\mathcal{R})$ or increase the data rate (multiplexing gain) for a given diversity order. Recent work [8] characterizes the fundamental tradeoff between the diversity and multiplexing gains as $\operatorname{div}\left(\nabla_{g}\right)=\left(a_{t}-\nabla_{g}\right) \times\left(a_{r}-\nabla_{g}\right)$ for a block fading channel with a length greater than $a_{t}+a_{r}-1$ [8]. The diversity order is represented by $\operatorname{div}\left(\nabla_{g}\right)$ and multiplexing gain by $\nabla_{g}$ for $a_{t}$ transmit and $a_{r}$ receive antennas where $\nabla_{g} \leq \min \left(a_{t}, a_{r}\right)$. In this case, a communication system can be defined by these two equations:

$$
\mathcal{R}=\nabla_{g} \log _{2}(1+\bar{\gamma}), \quad \text { and } \quad P_{e} \propto \frac{1}{\bar{\gamma} \operatorname{div}\left(\nabla_{g}\right)},
$$




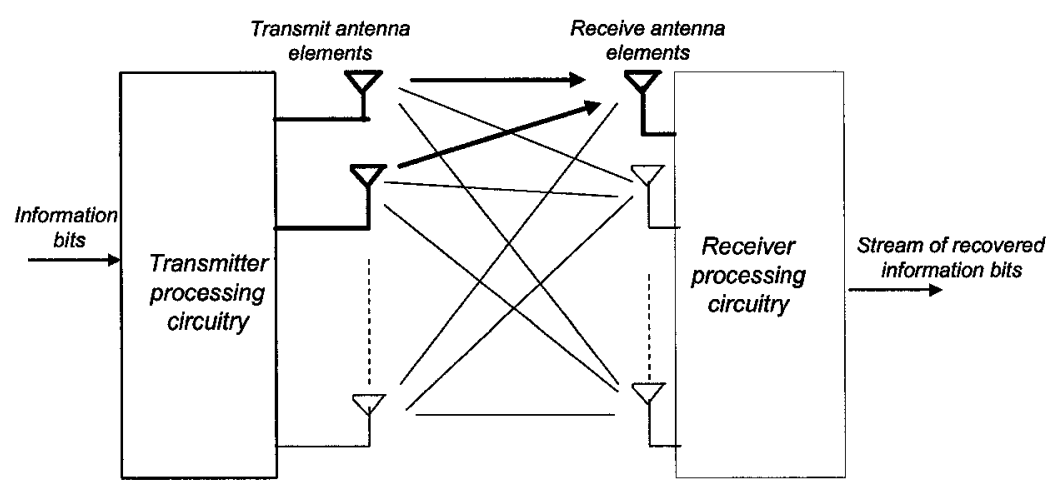

Figure 2.1: MIMO channels.

where $\bar{\gamma}$ is the signal-to-noise ratio (SNR) and $P_{e}$ is the probability of error.

This characterization implies that in a MIMO system, all transmit and receive antennas can be used for a diversity gain so that an error probability that is proportional to $1 / \bar{\gamma}^{a_{t} \times a_{r}}$ is obtained. Moreover, some of these antennas can be used to increase the data rate or multiplexing gain at the expense of the diversity gain.

The multiplexing gain approach uses $a_{t}$ transmit antennas to send $a_{t}$ independent data streams simultaneously to $a_{r}\left(a_{r} \geq a_{t}\right)$ receive antennas. This is done so that it realizes channel capacity as in the onion peeling of Bell Laboratories Layered Space-Time Architecture (BLAST) [9] or the diagonal encoding known as DBLAST.

The diversity gain approach is motivated by the work of [10] where the transmit diversity technique is shown to improve the signal quality at the receiver by simple processing across two transmit antennas. This technique offers the same diversity gains as those of the classical receiver diversity schemes. In terms of implementation, the transmit diversity scheme can easily be incorporated into existing wireless systems [11] such as indoor wireless LANs.

The capacity and diversity gains of MIMO system are further explored, using the generic system shown in Figure 2.1.

The input and output description of the MIMO channel can be expressed as

$$
\mathbf{y}=\mathbf{H x}+\mathbf{n},
$$


where $\mathbf{y}, \mathbf{H}, \mathbf{x}$, and $\mathbf{n}$ represent the received signal, channel samples matrix, transmitted signal vector, and additive noise, respectively. The entries of the channel matrix $\mathbf{H}$ can be modelled as an independently and identically distributed (IID) complex Gaussian random variable with zero mean and unit variance. The model in (2.2) indicates that the channel is constant over one frame (quasi-static channel assumption). The IID noise components $n_{k}$ have variance $\sigma_{k}^{2}$ and, hence, the average SNR of the system can be written as $\operatorname{tr}\left(E\left[\mathbf{x x}^{*}\right]\right) / \sigma^{2}$ which assumes that the channel gains are normalized and $\sigma^{2}=E\left[n_{k} n_{k}^{*}\right]$ for all $k$. The $E[\cdot]$ stands for the expectation operation, * denotes conjugate transpose and $\operatorname{tr}$ implies trace. The capacity of MIMO channel, conditioned on the $\mathbf{H}$ matrix, can be formulated as

$$
C=\ln \operatorname{det}\left(\mathbf{I}_{a_{r}}+\frac{\bar{\gamma}}{a_{t}} \mathbf{H H}^{*}\right) .
$$

For fixed channels, the evaluation of the channel capacity above is straightforward since the $\mathbf{H}$ matrix has fixed entries. This is not the case in wireless environments. For wireless channels, the capacity is associated with a given realization of $\mathbf{H}$ and, hence, it is a random quantity whose value is determined by $\mathbf{H}$. The channel capacity (C) can be obtained by averaging (2.3) over the distribution of $\mathbf{H}$. Considering the special case $a_{t}=a_{r}$, this capacity can be expressed as follows [12]

$$
\begin{aligned}
C & =E\left[\sum_{i=1}^{a_{t}} \ln \left(1+\frac{\bar{\gamma}}{a_{t}} \phi_{i}\right)\right] \\
& =a_{t} E\left[\ln \left(1+\frac{\bar{\gamma}}{a_{t}} \phi\right)\right],
\end{aligned}
$$

where $\left\{\phi_{i}\right\}$ are the random eigenvalues of $\mathbf{H}$. Equation (2.4) is expressed for identical eigenvalue situations $\phi_{i}=\phi, i=1,2, \cdots, a_{t}$. The probability density function of $\phi$ can be obtained as

$$
p(\phi)=\sum_{k=0}^{a_{t}-1} L_{k}^{0}(\phi)^{2} e^{-\phi},
$$

where $L_{k}^{0}$ is the Laguerre polynomial of order $k$. Thus, the capacity is [12]

$$
C=a_{t} \int_{0}^{\infty} \ln \left(1+\frac{\bar{\gamma}}{a_{t}} \phi\right) \sum_{k=0}^{a_{t}-1} L_{k}^{0}(\phi)^{2} e^{-\phi} d \phi \text { nats. }
$$




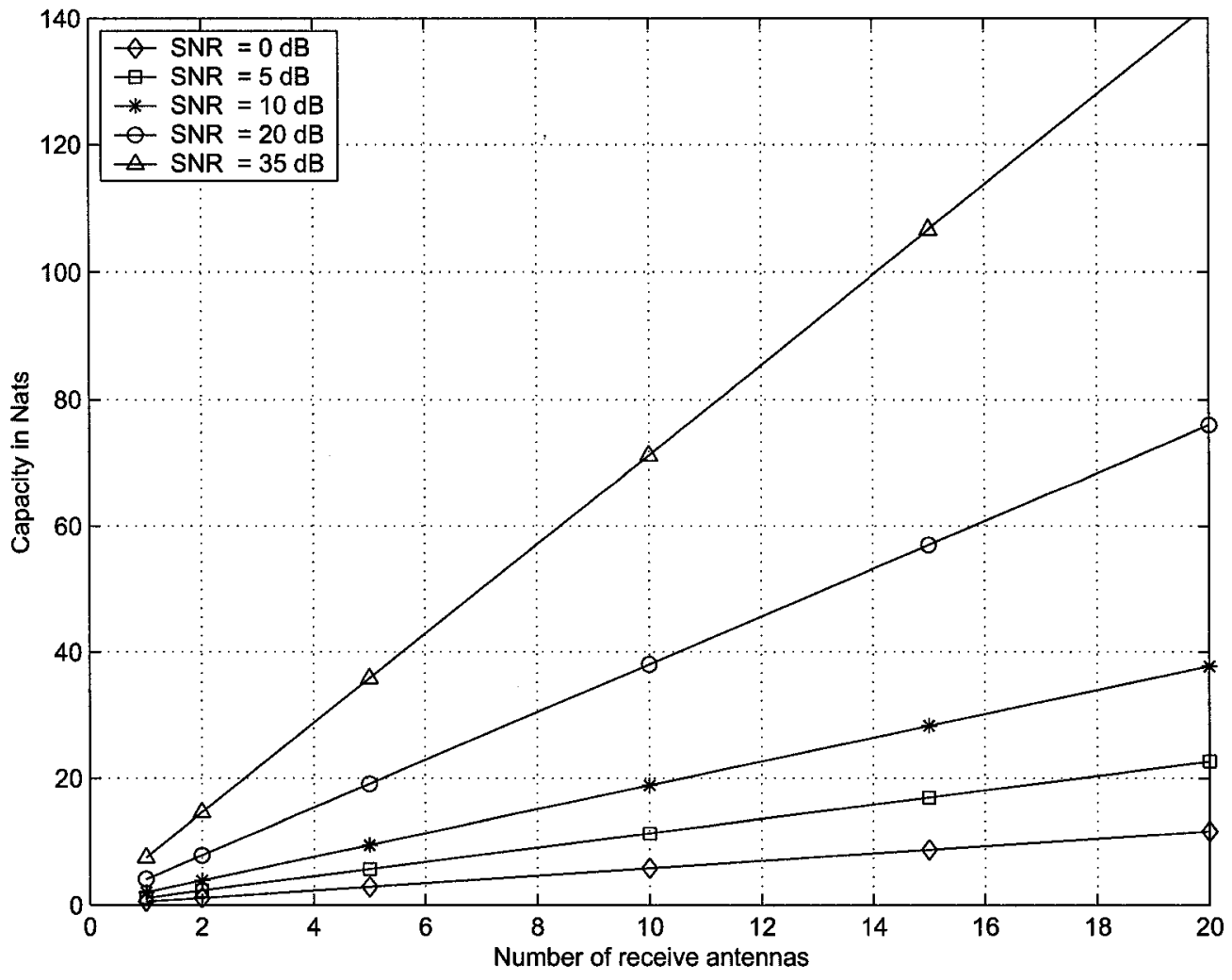

Figure 2.2: Capacity of a channel with $\left(a_{t}, a_{r}\right)$ MIMO for various value of SNR and $a_{t}=a_{r}$.

To gain insights into the capacity of MIMO, (2.6) is evaluated using numerical techniques for constrained power, $\bar{\gamma}=0,5,10,20,35 \mathrm{~dB}$. The capacity is observed to increase linearly with the number of antenna elements (Figure 2.2) as well as that an arbitrary capacity can be obtained with the MIMO systems. Space constraints, however, preclude the use of a large number of antenna elements on mobile terminals. In addition, any multi-antenna systems (MAS) with closely spaced antennas are affected by large scale fading known as shadowing.

\subsubsection{Macrodiversity}

Shadowing in wireless system falls under the category of macroscopic fading. This type of fading cannot be mitigated using collocated antennas since all the antennas are subjected 
to the same detrimental effects of shadowing. Macrodiversity techniques have been used to alleviate the shadowing effects through the reception of multiple signals at widely separated radio ports.

The conventional macrodiversity method involves serving a wireless unit with several base stations simultaneously, and selecting the one with the best signal quality. Selection combining yields the worst performance among the known combining techniques. A number of postdetection techniques, such as the multiply detected macrodiversity (MDM) [13, 14], are used to improve the performance of this conventional scheme. In MDM, all the received signals are detected in parallel at different base stations, and an algorithm is employed at the central unit to maximize the probability of correct decision.

In the recent years, new visions providing novel ways of handling signals received at widely separated antennas, such as the distributed antennas (DA) $[15,16]$, sectorized distributed antennas (SDA) [17], and radio-on-fiber (RoF) architectures [18, 19], have emerged. Such architectures are particularly suitable for microcellular systems where fiber links can conveniently connect these access points to a central unit. This topic will be revisited in a greater detail in Chapter 3.

\subsubsection{Microdiversity}

Spatial diversity is an effective way of mitigating the fading effects of wireless channels. This results in an increase in the spectrum efficiency or a boost in the system capacity. Various studies $[20,21,22]$ show that the capacity of the multipath environments and interferencelimited wireless systems can be increased using antenna techniques. Due to their large SNR gains, these techniques facilitate system design which are based on spectrally-efficient and multi-level constellation schemes. Multiple receive antennas can also be used as space-division multiple access (SDMA) to spatially separate the signals of different users, thus, providing a multiple-access gain. 
The work in [22] uses $L+\mathcal{N}_{u}-1$ antennas with optimum combining to adaptively cancel $\left(\mathcal{N}_{u}-1\right)$ co-channel interference in a wireless network containing $\mathcal{N}_{u}$ simultaneous users. At the same time the system provides $L$ diversity order for each of the $\mathcal{N}_{u}$ users. This means that the user's error probability decreases as the $L^{t h}$ power of the SNR $\bar{\gamma}$ (i.e., $P_{e} \propto 1 / \bar{\gamma}^{L}$ ) in addition to obtaining $\mathcal{N}_{u}$-fold increases in user capacity.

Once the diversity signals are provided, there are many ways to combine and/or utilize them. The choice of combining methods depends essentially on complexity restrictions and the amount of channel state information available to the receiver [23]. The classical techniques are maximal ratio combining (MRC), equal-gain combining (EGC), and selection combining (SC). More recently, generalized selection combining (GSC) has been introduced [24], analyzed [24, $25,26,27]$ and the cutoff rate presented for Rayleigh fading [28]. Of all the combiners, MRC provides optimum performance in non-interference channels and is also the most complex one to implement. Its operation depends on the perfect knowledge of the channel, meaning that the estimates of the channel gains (attenuation and phase shifts) do not contain noise. The probability of error for $L$-diversity scheme using BPSK in Rayleigh fading channels, is derived on the basis of this assumption as [29]

$$
P_{b}(E)=\left(\frac{1-\mu_{c}}{2}\right) \sum_{l=0}^{L-1}\left(\begin{array}{c}
L-1+l \\
l
\end{array}\right)\left(\frac{1+\mu_{c}}{2}\right)^{l}
$$

where $\mu_{c}=\sqrt{\frac{\bar{\gamma}}{1+\bar{\gamma}}}$ and $\bar{\gamma}$ is the average SNR per bit. Asymptotically, this performance can be approximated as

$$
P_{b} \approx\left(\frac{1}{4 \bar{\gamma}}\right)^{L}\left(\begin{array}{c}
2 L-1 \\
L
\end{array}\right)
$$

where the diversity order is seen as $L$ since $P_{b} \propto 1 / \bar{\gamma}^{L}$. Equation (2.7) is plotted in Figure 2.3. A gain of about $29 \mathrm{~dB}$ is obtained with $L=5$ over non-diversity channel $(L=1)$ at an error rate of $10^{-4}$. This represents a factor of about $800\left(10^{2.9}\right)$ reduction in the system power requirement. 


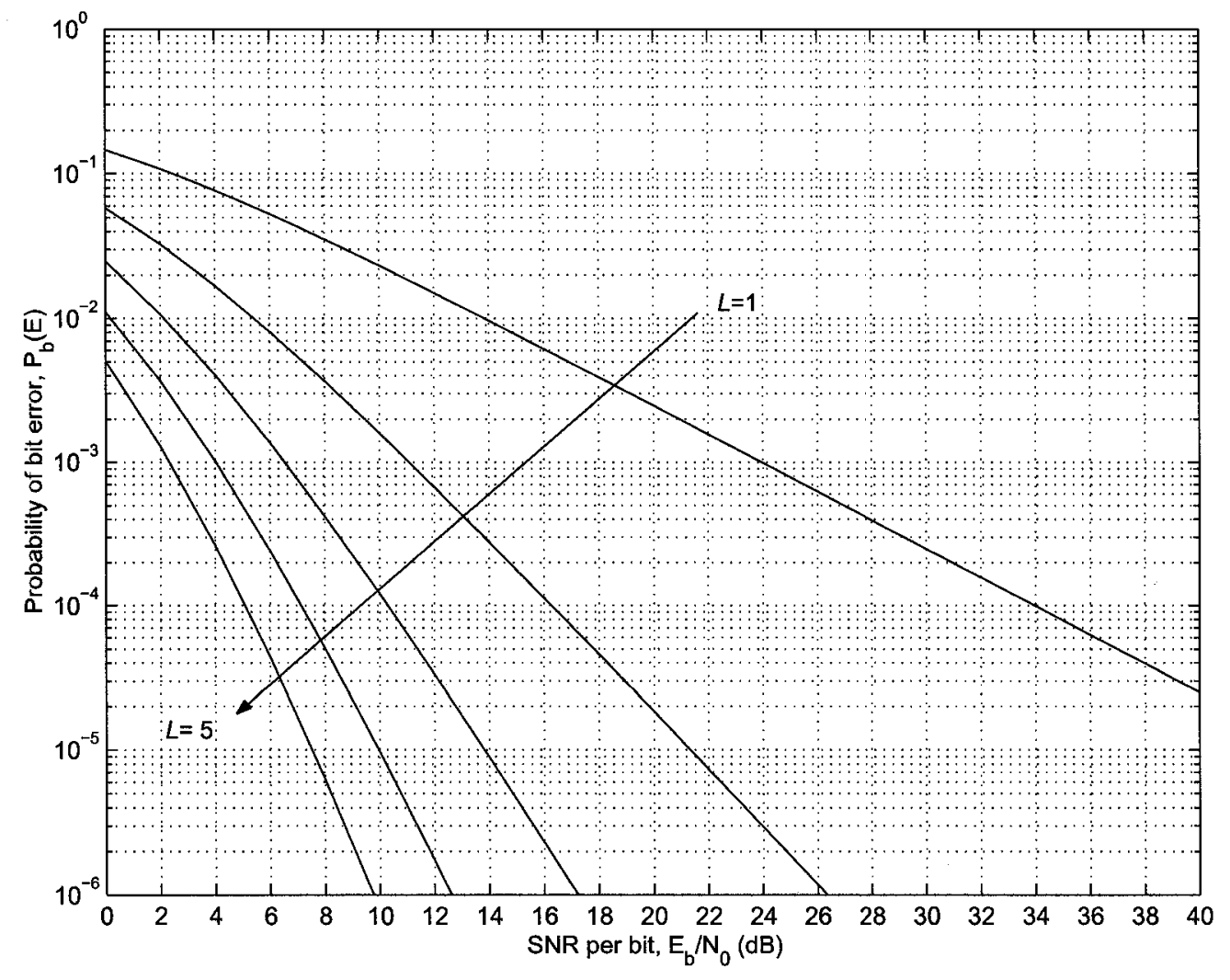

Figure 2.3: Performance of binary signals with MRC in Rayleigh fading channels. 
Diversity signals can easily be created but there are two limitations related to the power requirement and the cost of $\mathrm{RF}$ electronics for each diversity branch required to process the diversity signals. For example, a large order of antenna diversity could be obtained at high frequency bands using spatial separation and/or orthogonal polarization [27]. The use of wideband signal where the signal bandwidth $W$ is made much greater than the channel coherence bandwidth can also be employed to provide diversity. Such signal bandwidth resolves the multipath components with fine time resolution of $(1 / W)$. Thus, the receiver is provided with several independent fading signal paths. Ultra-wide band transmission and CDMA systems use this technique and this makes them multipath resistant.

For example, by using $1 \mathrm{GHz}$ bandwidth in ultra-wide bandwidth transmissions, a large number of paths can be created with differential path delay resolution of less than a nanosecond [30]. The optimum algorithm to extract diversity in all these scenarios is the rake receiver [31]. The rake receiver gathers all the resolved signal paths. However, the question is whether we can always utilize all of these signals or all of the diversity branches created in the spatial antenna case.

Given that the RF chains constitute the bulk of the receiver cost, it may not be cost-efficient to provide separate RF chains to all of the created diversity paths. In view of this, the GSC receivers have become popular as they provide most of the diversity benefits at a reasonable cost. In the GSC reception of signals, a subset of diversity branches is selected and combined as in MRC [24]. In addition, due to the multitude of resolvable signals in UWB systems, the notion of maximum-energy-capture selective rake (SRake) receiver is introduced [30]. The GSC and SRake processors are a compromise to the MRC and all rake receiver complexities, respectively.

The discussion so far indicates that the wireless systems continue to depend on multiantenna techniques. These schemes yield considerable gains both in SNR and system capacity over single antenna systems. However, the consistently decreasing and miniaturized terminals 
pose obstacles to exploiting the benefits of these techniques. In view of this, cooperative relaying techniques are necessary.

\subsection{Relaying and the Notion of Cooperative Diversity}

To overcome the drawbacks of spatial limitations of wireless terminals, distributed network nodes (could be active terminals or fixed relays which are deployed by the service providers) can be engaged in cooperative fashion to emulate antenna arrays. This new technique is fundamentally different from collocated arrays. The (virtual) antenna elements in the cooperative schemes are widely separated and connected through wireless links in contrast to the traditional connection made through physical cablings.

\subsubsection{Relaying and Multihop Techniques}

The traditional cellular radio network is a single hop communication network where wireless terminals communicate directly with a single fixed base station as shown in Figure 2.4 (a). However, most times terminals could be outside the footprint of their base stations or located in unfavorable conditions resulting in coverage dead spots. It can be caused by a number of scenarios such as a user being in a deep valley shadowed by a mountain or a man-made structure, in an underground shopping mall, subway station or tunnel. These problems would increase in future generation wireless networks as these systems would have to support ubiquitous high data rate service. Relaying or multi-hop techniques are promising candidates for deploying these networks in a cost effective way. Multi-hop implies that the signal of the source passes through one or more intermediate nodes (relays) towards its destination.

The following link budget calculation example might help explain the coverage extension and/or terminal transmit power reduction ability of relays. Consider that a capacity (C) of $400 \mathrm{Mb} / \mathrm{s}$ is desired in a $5.3 \mathrm{GHz}$ carrier frequency system with access to $100 \mathrm{MHz}$ bandwidth 


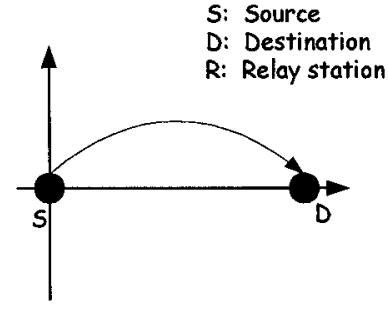

(a). Conventional single-hop network

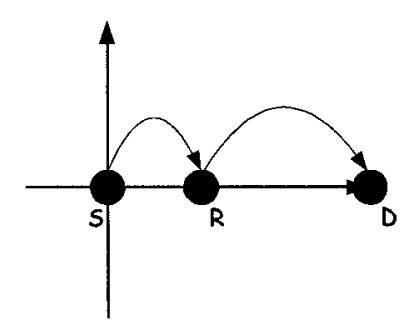

(b). Conventional two-hop relay network

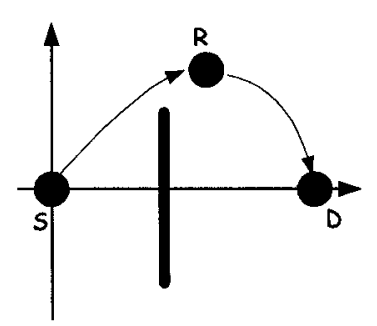

(c). Combatting shadowing

Figure 2.4: Conventional relay networks, (a) conventional single-hop network, (b) conventional two-hop relay network, (c) combatting shadowing with a relay.

$(W)$ and a noise level of $-94 \mathrm{dBm}$. The Shannon formula $C=W \log _{2}(1+S N R)$ dictates that a SNR of $12 \mathrm{~dB}$ is required. A terminal is supportable (not in outage) if the received signal level is at least $-82(=-94+12) \mathrm{dBm}$. The received signal can be expressed as [32]

$$
\mathcal{P}_{r}=\mathcal{P}_{t}+G_{a}-P L-S_{\text {loss }}
$$

where $\mathcal{P}_{t}, \mathcal{P}_{r}, G_{a}$, and $S_{\text {loss }}$, represent transmit power, receive power, antenna gains, and system losses respectively. Consider $\mathcal{P}_{t}=50 \mathrm{~mW}(17 \mathrm{dBm})$ and $S_{\text {loss }}=0, G_{a}=7 \mathrm{~dB}$. Using a simple path loss model ${ }^{1} P L=-20 \log _{10}(c / 4 \pi f r)$ where $c$ is the speed of the light and $r$ the distance between communicating nodes, two terminals $T_{1}$ and $T_{2}$ located at distance of 100 and 450 meters respectively from the base station, experience path loss $P L=87$ and 100 dB respectively.

The received power level $\left(\mathcal{P}_{r}\right)$ of Terminal $T_{1}$ is $-77 \mathrm{dBm}$ while that of $T_{2}$ is $-90 \mathrm{dBm}$. In this illustration, an optimistic channel scenario is assumed. Other channel impairments such as interference, multipath, etc, are neglected. Terminal $T_{2}$ is in outage since the received power is $8 \mathrm{~dB}$ below the required SNR. Traditionally, a bigger cell can be split into smaller ones to help decrease the path loss experienced by terminals in the same condition as terminal $T_{2}$.

\footnotetext{
${ }^{1}$ Commonly called free space path loss model.
} 
However, from (2.9) intermediate relays can be used to break or reduce the overall path loss. In addition, a higher antenna processing gain $G_{a}$ can be used to improve $\mathcal{P}_{r}$. Conventionally, this gain is obtained using large antenna arrays. It happens that this array with large number of antennas can be built in a virtual way through distributed fixed relay stations by using cooperative protocols. Furthermore, for a given $\mathcal{P}_{r}$, the path loss savings and antenna processing gains can be used to reduce the terminal transmit power. Relaying splits longer paths into shorter segments that have the effect of reducing the overall path loss which is a consequence of the non-linearity of the path loss [33].

The above discussion demonstrates that the common use of relays in communication networks reduces path loss (Figure 2.4 (b)) as well as combats shadowing (Figure 2.4 (c)). This thesis investigates a cooperation between relays and the source. The cooperative relaying schemes, therefore, represent an add-on to conventional relaying since in principle these relays are expected to be deployed by service provider for the usual traditional usage (Figure 2.4 (b) and Figure $2.4(\mathrm{c}))$. At the same time, cooperative relaying represents a paradigm shift from point-to-point coding to holistic network coding.

\subsubsection{Relaying Classification}

Based on the retransmission mechanism, the wireless relaying techniques are classified into two main types decode-and-forward (DF) and amplify-and-forward (AF) [34]. In the DF relaying, the relay regenerates the received signal (full detection), reprocesses and retransmits it to the destination. For the AF relays, the relay behaves like analog repeater, it simply amplifies the received signal and forwards it.

Mobile (terminal) and fixed relaying are classified in the literature based on mobility and topology. Terminal relaying uses mobile terminals as relays to pass signals between base stations and other mobile terminals while fixed relaying is based on fixed relay nodes deployed as a part of the network infrastructure. The advantages of terminal relaying include reduced 
infrastructure cost and good potential coverage because of the large number of terminals in the network. However, there are some drawbacks such as increased cost and complexity of user equipment. Each terminal must be modified to obtain relaying capability. Complex routing protocols are required.

Relaying using fixed entities, on the other hand, involves initial deployment cost. This incremental cost is offset by reduced requirements on the mobile terminals since simple and less stringent routing algorithms and protocols are involved. At the same time relay deployment is flexible as relays can be moved to accommodate traffic patterns [35]. Furthermore, these fixed relay stations are a part of the entire cellular network infrastructure which implies that their deployments are performed jointly with the network design and planning.

The integration of multihop capability in the new wireless systems constitutes significant economical advantage compared to the cell splitting, often used to increase the system capacity and spectral efficiency [37]. In cell splitting, each new cell requires the deployment of expensive base stations and new connections to its wired backbone as well as it involves numerous problems associated with fast handoffs. Instead of new base stations, the relaying approach deploys wireless relays that are used to establish multihop networks. These relays do not require any wired connection to the backhaul but wirelessly re-route received signal to either the BS (uplink) or user (downlink) depending on the case.

The elimination of the backplane that serves as an interface between the BS and the wired backhaul network [36] therefore results in substantial savings. The relaying techniques are excellent candidates for providing the quantum leap into the ambitious performance (coverage, capacity, and reliability) expected of the future generations of wireless systems. The interest in this area is growing steadily. This is in conjunction with the new paradigm which is interchangeably called antenna sharing [38, 40], cooperative diversity [39], or virtual antenna arrays [52]. 


\subsubsection{Virtual Antenna Arrays}

The virtual antenna array (VAA) $[52,53]$ technique is a pragmatic approach to communication network design in which communicating entities utilize each other's resources in a symbiotic manner. The principle for deploying VAA in cellular networks is explained for the downlink. It is assumed that a base station uses its antenna arrays to transmit a space-time encoded data stream to a group of mobile terminals forming a virtual array group. Each terminal receives the stream of data meant for this group it extracts its data and relays the data meant for the other members. Each of the terminals receives copies of its signal from its colleagues in their group. After receiving from other partners in the VAA group, each terminal can start to process all the signal copies. Since the terminal receives independent copies of its signal from other terminal, these signals can be carefully processed to yield diversity gains.

Although the VAA concept promises a drastic increase in capacity, it brings new challenges as well. It assumes that the terminal can receive and transmit simultaneously on the same channel (full duplex) ${ }^{2}$. This duplex mode may pose serious constraints on the mobile terminal radio frequency chain as the receive and transmit signals have to be adequately decoupled (using very sensitive filters) or a sufficient frequency separation should be allowed between receiving and transmitting bands of terminal relays. It is not a simple relay problem since each user has to transmit its partner's information along with its own. It needs, therefore, to reliably detect or intelligently amplify the signal of its partner. Hence, this scheme calls for stringent data scheduling and strict synchronization of users.

\subsubsection{Cooperative Networks}

The idea behind cooperative communication dates back to the work of Meulen [54], and Cover and El-Gamal [55] on relay channels. Their focus however, is theoretical expositions rather than practical implementation. Reference [55] derives the maximum achievable rate

\footnotetext{
${ }^{2}$ In practice, this is done on two orthogonal frequency channels.
} 


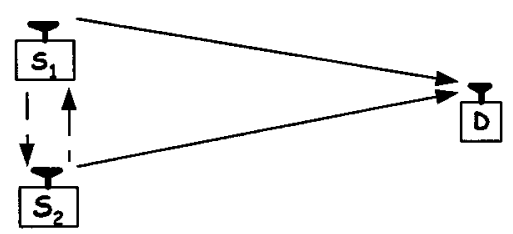

Figure 2.5: User cooperation.

for cases with or without feedback to the source or relay terminal for Gaussian channels in a way which does not appear to have direct application to wireless relay networks. Their treatment, for example, assumes that relays can receive and transmit at the same time in the same band which might be difficult to achieve in inexpensive networks. Nevertheless, their work provides the basis for the cooperative schemes and protocols $[38,39]$ that recently have gained prominence.

Sendonaris, Erkip, and Aazhang [39] present a simple exposition of user cooperation diversity scheme which in its simplest form stipulates that two users form a partnership to transmit each other's signals to the base station. First, each of the partners exchanges cooperative information. Each partner detects this cooperative signal and transmits it along with its (own) signal to the destination. This form of cooperation leads to an increase in capacity for both users as well as provides robust user achievable rate, i.e, rate that is less susceptible to channel variations. The authors show the implementation of their approach to practical systems such as CDMA. The success of this cooperative scheme largely depends on the interuser channels shown in Figure 2.5. The interuser channel is the path from the output of each encoder to the input of its partner.

The work of Laneman and Wornell [38] presents a similar treatment but in a conceptual manner, puts the user cooperation in a mathematical framework. It discusses a cooperative protocol for combating multipath fading of wireless networks by exploiting the spatial diversity 
available among terminals that have agreed to assist each other. The proposed protocol helps to remove some of the practical obstacles of earlier work.

The low complexity cooperative protocols recognize that for practical reasons, terminals cannot receive and transmit at the same time on the same channel. This is referred to as an orthogonality constraint. These complexity challenges, therefore, impose half-duplex as opposed to full duplex communication of the initial relay work [55]. The same constraint can be exploited to facilitate cooperation through resource allocation. For instance, in direct transmission mode all terminals occupy the entire time slot simultaneously causing multiple access interference (MAI), as indicated in Figure 2.6 (a). Figure 2.6 (b) shows how orthogonal time slots can be used to alleviate MAI in non cooperating terminals, while Figure 2.6 (c) shows time slot allocations for cooperating terminals. Though the cooperating terminals transmit and receive at different times, the overall transmit time for each terminal is the same as in Figure 2.6 (b), establishing a basis for comparing cooperative cases. This illustration shows the ease at which this protocol could fit into TDMA-based systems. The cited literature demonstrates that cooperation yields full spatial diversity, allowing significant power savings at a given performance criterion, all things being equal. The referred studies [38, 39] have mainly used outage probability as the figure of merit.

A typical cooperative relaying network is shown in Figure 2.7. For conventional relaying, the destination ignores the direct signal by relying only on the signal received through the relay (Figure 2.4 (b)). In contrast, the cooperative relaying scheme exploits the broadcast nature of wireless systems. This means that, the relay as well as the destination hear the source (Figure 2.7). The DF fixed protocol approach allows the relay (terminal) to always decode and forward to the destination. This relay link could propagate errors, degrading the spatial diversity gains desired from this relay's cooperation. To exploit the full benefit of spatial diversity in cooperative communication, participating relays should not always forward the received signal [45] to prevent error propagation, as this has a negative impact on cooperation 


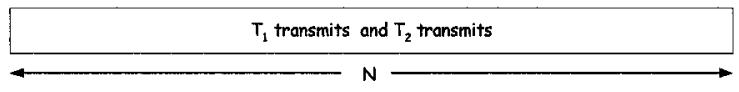

(a)

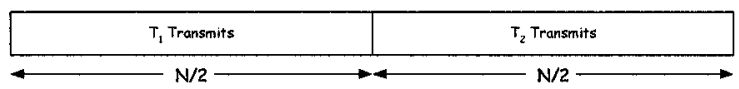

(b)

\begin{tabular}{|l|c|c|c|}
\hline$T_{1}$ Transmits $+T_{2}$ receives & $T_{2}$ Relays & $T_{2}$ Transmits $+T_{1}$ receives & $T_{1}$ Relays \\
\hline
\end{tabular}

(c)

Figure 2.6: User cooperation and resource sharing (a) direct, multiple access transmission (MAI), terminals $T_{1}$ and $T_{2}$ transmit at the same time, (b) orthogonal direct transmission, (c) orthogonal cooperative diversity.

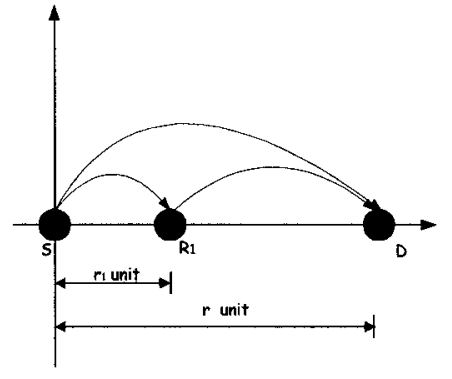

(a)

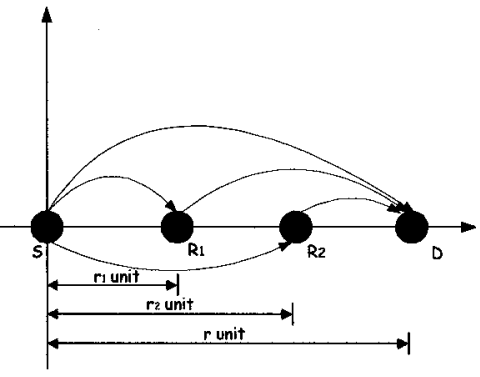

(b)

Figure 2.7: Network configuration for parallel relays: (a) single cooperating terminal, (b) two cooperating terminals.

schemes.

Most of the previous work uses repetition-based protocols for relaying. Some even employ automatic repeat request schemes in order to improve error performance at the expense bandwidth. The inefficient use of bandwidth of repetition-based protocols calls for more prudent schemes. For example, coded cooperative schemes, where signals are not repeated, are proposed [51, 56, 57]. Hunter and Nosratinia [51] proposed a coded cooperation scheme that partitions the $N$-bit long codeword of each user into two frames $N_{1}$ and $N_{2}$. This partitioning 
can be done by puncturing techniques. Each user transmits the $N_{1}$-bit frame to its partner, which decodes and then formulates the punctured (deleted) bits. These bits constitute the second frame $N_{2}$. The users then transmit the $N_{2}$-bit frame of their partner to the base station. The base station combines the two frames $N_{1}$ and $N_{2}$ to form the original $N$-codeword. Since the $N_{1}$ and $N_{2}$ frames for each user are received through independent channels, diversity can be exploited. During the transmission of the partner's parity frame $\left(N_{2}\right)$, the user does not transmit data of its own.

There are many questions of practical importance that arise. The scheme imposes a specific coding scheme on the mobile terminals. It is possible that one or none of the users decode correctly (in very noisy interuser channels). Both users are assumed to transmit to the base station at the same time, which is a recipe for interference. Some of these issues are partly addressed in [57], where the authors propose code design that ensures a reliable interuser channel. They achieve this by optimizing the rate compatible punctured code used in [51]. Extension to space-time coded cooperation is conducted in [57]. The space-time component is required to extract diversity in fast fading environments. This also makes it possible for a cooperating user to transmit its signals along with that of its partner's parity.

The following section presents the channel models that are employed in evaluating the performance of the proposed systems. This is followed by some derivations related to the channel distribution utilized in subsequent chapters.

\subsection{Models for Fading Channels}

Wireless signals are unguided due to the terrain and nature of signal propagation. These signals experience fading where both the envelope and phase of the received signals fluctuate over time. In the discussion in this thesis, it is assumed that coherent modulation and perfect phase recovery is possible. Therefore, the performance analysis over fading channels requires only fading envelope statistics. Since, it is assumed that the fading is sufficiently slow, the 
fading envelope random process can be represented by a random variable over any given symbol period.

\subsubsection{Fading Models}

In fading channels, the received signal is modulated by the fading amplitude $\alpha$, where $\alpha$ is a random variable (RV) with mean square value $\Omega=E\left[\alpha^{2}\right]$. At the receiver the faded signal is perturbed by additive white Gaussian noise (AWGN) which is usually assumed to be statistically independent of the fading amplitude and also spatially independent from antenna branches. The noise is characterized by one-sided power spectral density $N_{0}(\mathrm{~W} / \mathrm{Hz})$. Thus, the instantaneous signal-to-noise power ratio (SNR) per symbol denoted by $\gamma=\alpha^{2} E_{s} / N_{0}$ and the average SNR per symbol by $\bar{\gamma}=E\left[\alpha^{2} E_{s} / N_{0}\right]=\Omega E_{s} / N_{0}$, where $E_{s}$ is the energy per symbol. With the PDF of $\alpha, p(\alpha)$, the PDF of the RV $\gamma$ is obtained using the method of change of variable for a function of a RV [23]

$$
p_{\gamma}(\gamma)=\frac{\left.p_{\alpha}(\alpha)\right|_{\alpha=\sqrt{\Omega \gamma / \bar{\gamma}}}}{2 \sqrt{\frac{\bar{\gamma} \gamma}{\Omega}}}=\frac{p_{\alpha}\left(\sqrt{\frac{\Omega \gamma}{\bar{\gamma}}}\right)}{2 \sqrt{\frac{\bar{\gamma} \gamma}{\Omega}}} .
$$

The Rayleigh distribution is frequently used to describe the statistical fluctuations of signals received from multipath fading. This multipath fading is due to the constructive and destructive combination of randomly delayed, scattered, reflected, and diffracted signal components. A versatile statistical model often used to model a wide range of fading environments which has shown to be quite appropriate in modeling multipath fading in mobile radio channels is the Nakagami- $m$ distribution [75] with the following PDF:

$$
p_{\alpha}(\alpha, m)=\frac{2 m^{2} \alpha^{2 m-1}}{\Gamma[m] \Omega^{m}} \exp \left(-\frac{m \alpha^{2}}{\Omega}\right), \quad \alpha>0,
$$

where $m$ is known as Nakagami parameter that specifies the severity of the fading and $\Gamma[\cdot]$ denotes the gamma function. For instance, the Rayleigh fading distribution may not be realistic in microcellular network where a line-of-sight is probably going to exist. In such 
a situation, a channel model with a line-of-sight component such as the Ricean model is preferred. In Ricean fading, the received signal is composed of two components, a scattered and a direct part with power of $A$ and $\sigma_{r}^{2}$, respectively. These power components define a Ricean $\mathcal{K}$ - factor given as $\mathcal{K}=A^{2} / 2 \sigma_{r}^{2}$. This channel model is related to the Nakagami through the transformation $\mathcal{K}=\frac{\sqrt{m^{2}-m}}{m-\sqrt{m^{2}-m}}[37]$. Note that $m=1$ reduces $(2.11)$ to the Rayleigh distribution. In this thesis, the Nakagami model is used in most of the presented numerical examples.

The PDF of $\gamma$ for the Nakagami distribution can be obtained by applying (2.10) on (2.11) to arrive at

$$
p_{\gamma_{i}}\left(\gamma_{i}, m_{i}\right)=\frac{m_{i}^{m_{i}} \gamma_{i}^{m_{i}-1}}{\bar{\gamma}_{i}^{m_{i}} \Gamma\left[m_{i}\right]} \exp \left(-\frac{m_{i} \gamma_{i}}{\bar{\gamma}_{i}}\right), \quad \gamma_{i} \geq 0, \quad m_{i} \geq 1 / 2
$$

where $\gamma_{i}=\alpha_{i}^{2} E_{s} / N_{0}$ and $\bar{\gamma}_{i}=E\left[\alpha_{i}^{2} E_{s} / N_{0}\right]=\Omega_{i} E_{s} / N_{0}$ is the average SNR per symbol for the $i$-th diversity branch.

If we set $m=1$, the distribution of the SNR per symbol is the exponential distribution, which represents the distribution of the SNR in the Rayleigh faded signal [29]

$$
p_{\gamma}(\gamma)=\frac{1}{\bar{\gamma}} \exp \left(-\frac{\gamma}{\bar{\gamma}}\right)
$$

Finally, a unified fading parameter referred to in [84] as the amount of fading (AmF) is mentioned, which serves as a measure of the severity of fading associated with any fading PDF. AmF is defined as

$$
\mathrm{AmF}=\frac{\operatorname{Var}\left[\alpha^{2}\right]}{\left(E\left[\alpha^{2}\right]\right)^{2}}=\frac{E\left[\gamma^{2}\right]-(E[\gamma])^{2}}{(E[\gamma])^{2}}
$$

where $\operatorname{Var}[\cdot]$ is the variance. The moments $E\left[(\cdot)^{p}\right]$ for the Nakagami PDF given above can therefore be derived as

$$
E\left[\gamma^{p}\right]=\frac{\Gamma(m+p)}{\Gamma(m) m^{p}} \bar{\gamma}^{p},
$$

which follows by using (2.14) that the AmF for the Nakagami distribution is

$$
\mathrm{AmF}_{m}=\frac{1}{m}, \quad m \geq 1 / 2
$$


The Rayleigh fading therefore has an $\mathrm{AmF}$ equal to 1. It can be seen that Nakagami-m distribution covers a wide range of $\mathrm{AmF}$ through the $m$ parameter. For instance, one-sided Gaussian distribution is obtained by setting $m=1 / 2$ and in the limit as $m \rightarrow+\infty$, the Nakagami- $m$ fading channel converges to the AWGN channel demonstrating the versatility of this fading model.

The cumulative distribution function (CDF) of the fading process is an important channel characteristic. This CDF is obtained as $F_{\gamma}(\gamma)=\int_{0}^{\gamma} p_{\gamma}(\xi) d \xi$. Hence, for the Nakagami fading this can be evaluated as

$$
F_{\gamma}(\gamma, m)=\left(\frac{\gamma}{\bar{\gamma}}\right)^{m} \frac{m^{m-1}}{\Gamma(m)}{ }_{1} F_{1}\left([m],[1+m],-\frac{\gamma m}{\bar{\gamma}}\right),
$$

where ${ }_{1} F_{1}(a, b, z)$ is the confluent hypergeometric function [80, pp. 1085 eqn. (9.210.1)] which can be obtained from the series expansion ${ }_{1} F_{1}(a, b, z)=1+\frac{a z}{b}+\frac{a(a+1) z^{2}}{b(b+1) 2 !}+\cdots=\sum_{k=0}^{\infty} \frac{(a)_{k}}{(b)_{k}} \frac{z^{k}}{k !}$, where $(a)_{k}$ is the Pochhammer symbol denoted as Pochhammer $[a, k][81]$, and $(a)_{k}=\frac{\Gamma(a+k)}{\Gamma(a)}$. Using this definition of the ${ }_{1} F_{1}(a, b, z)$, we can express

$$
\begin{aligned}
{ }_{1} F_{1}\left([m],[1+m],-\frac{\gamma m}{\bar{\gamma}}\right) & =1+\frac{m}{m+1}\left(\frac{-\gamma m}{\bar{\gamma}}\right)+\frac{m(m+1)}{2 !(m+1)(m+2)}\left(\frac{-\gamma m}{\bar{\gamma}}\right)^{2} \\
& +\frac{m(m+1)(m+2)}{3 !(m+1)(m+2)(m+3)}\left(\frac{-\gamma m}{\bar{\gamma}}\right)^{3}+\cdots \\
& =\sum_{k=0}^{\infty} \frac{1}{k !} \frac{m}{m+k}(-1)^{k}\left(\frac{\gamma m}{\bar{\gamma}}\right)^{k} \\
& =m\left(\frac{\gamma m}{\bar{\gamma}}\right)^{-m} \sum_{k=0}^{\infty} \frac{1}{k !} \frac{1}{m+k}(-1)^{k}\left(\frac{\gamma m}{\bar{\gamma}}\right)^{k+m}
\end{aligned}
$$

Putting (2.18) in (2.17) and using [80, pp. 950 eqn. (8.354.1)], (2.17) can be expressed as

$$
F_{\gamma}(\gamma, m)=\frac{1}{\Gamma(m)} \sum_{k=0}^{\infty} \frac{(-1)^{k}}{k !(m+k)}\left(\frac{\gamma m}{\bar{\gamma}}\right)^{k+m}=\frac{\Gamma_{\mathrm{L}}\left[m, \frac{m \gamma}{\bar{\gamma}}\right]}{\Gamma(m)}
$$

where $\Gamma_{\mathrm{L}}[a, b]=\int_{0}^{b} e^{-t} t^{a-1} d t$ denotes the incomplete gamma function. For $m=1, F_{\gamma}(\gamma, 1)=$ $\Gamma_{\mathrm{L}}\left[1, \frac{\gamma}{\gamma}\right]$. The definition of the incomplete gamma function can be used to show that ${ }^{3}$,

$$
F_{\gamma}(\gamma, 1)=1-\exp \left(-\frac{\gamma}{\bar{\gamma}}\right)
$$

\footnotetext{
${ }^{3}$ See Appendix C.1.
} 


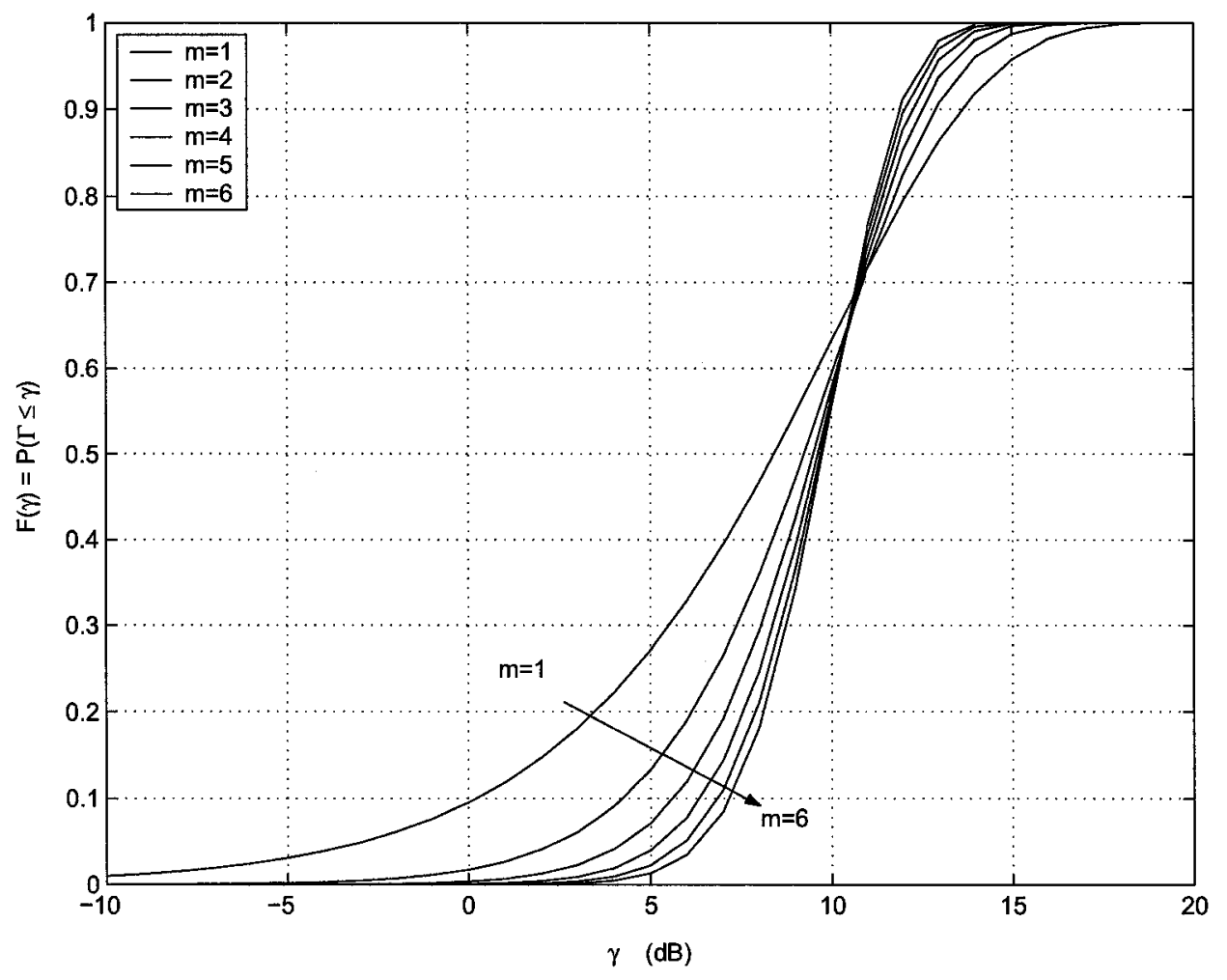

Figure 2.8: The CDF of Nakagami distribution with different values of $m$.

is the CDF of the exponential distribution shown in (2.13). Figure 2.8 shows the CDF of the Nakagami fading for different values of the parameter $m$. In this figure the average assumed SNR is $10 \mathrm{~dB}$ and the channel power is normalized (set to $0 \mathrm{~dB}$ ).

In Chapter 4 a threshold decoding strategy at the relay to reduce the error propagation is introduced. In this strategy the relay decodes and forwards only when the instantaneous received SNR is larger than a predetermined SNR threshold, $\gamma_{t h}$. Note that there are other means by which the error propagation can be reduced. One common technique is the use of cyclic redundancy check (CRC) in frame-based communication. Assuming a single channel (no diversity) link the relay decodes and forwards only when $\gamma \geq \gamma_{t h}$. The probability of 


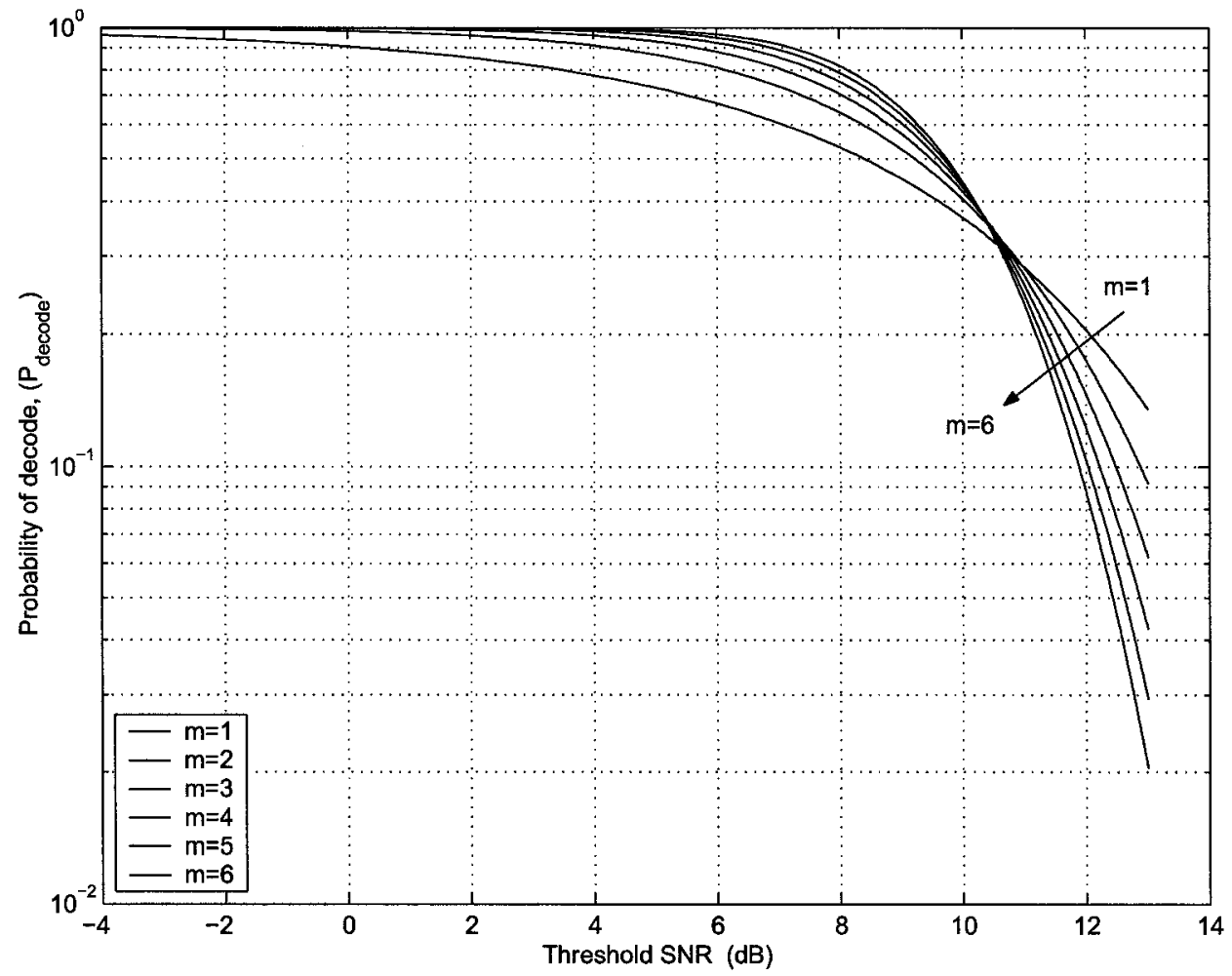

Figure 2.9: Probability of decoding in threshold-based decode-and-forward protocol.

decode is therefore $\operatorname{Pr}\left(\gamma \geq \gamma_{t h}\right)$. This probability is represented as

$$
\begin{aligned}
P_{\text {decode }} & =\int_{\gamma_{t h}}^{\infty} p_{\gamma}(\xi, m) d \xi \\
& =1-\frac{\Gamma_{\mathrm{L}}\left[m, \frac{m \gamma_{t h}}{\bar{\gamma}}\right]}{\Gamma(m)},
\end{aligned}
$$

where $\bar{\gamma}=\Omega_{\mathrm{sr}} E_{s} / N_{0}$ and $\Omega_{\mathrm{sr}}$ is the channel fading average power.

Some illustrations of the effect of $m$ on the probability of decode in threshold-based decode and forward relay is shown in Figure 2.9 for an average received SNR of $10 \mathrm{~dB}$. It is obvious that the decoding threshold should be determined taking into account the amount of fading.

The fading channel model assumes that frequency flat fading is encountered. This model assumes that all the spectral components of the transmitted signal are affected in a similar manner. Otherwise, the frequency selective model can be adopted; typically used in wide- 
band systems where the transmitted bandwidth is larger than the coherence bandwidth of the channel. The above presented model allows for analysis of the performance of various communication systems operating over these channels.

\subsubsection{Decode-and-Forward Probability in Multi-antenna Systems}

The aim in threshold decoding is to ensure that a signal forwarded by the relay is reliable since the number of times reliably detected signal is relayed to the destination has an impact on the cooperation benefit. Therefore, the decode-and-forward probability (DFP) of the relay is an important system performance criterion. This logically leads to examining the DFP of the multi-antenna relay with a threshold DF strategy. The examination begins with SC where the relay first selects the branch with the largest instantaneous SNR, i.e., $\gamma=\max \left[\gamma_{1}, \cdots, \gamma_{L}\right]$ and then compares it to the set decoding threshold $\gamma_{t h}$. The joint probability density function (PDF) of selecting $n$ largest from $L$ independently and identically distributed random variables is given in a general form in [79] as $p_{\gamma_{1}, \cdots, \gamma_{n}}\left(\gamma_{1}, \cdots, \gamma_{n}\right)=n ! C_{n}^{L}[F(\gamma)]^{L-n} \prod_{l=1}^{n} p\left(\gamma_{l}\right)$ where $F(\gamma)$ is the cumulative distribution function (CDF). The selection of the best branch in terms of instantaneous SNR is performed. For this reason, for the underlying Nakagami- $m$ distribution, the required PDF is reduced to $p(\gamma, m)=L[F(\gamma, m)]^{L-1} p_{\gamma}(\gamma, m)$ as

$$
p(\gamma, m)=\frac{L m^{m} \gamma^{m-1} \exp \left(-\frac{m \gamma}{\bar{\gamma}}\right)}{\bar{\gamma}^{m}(\Gamma[m])^{L}}\left(\sum_{k=0}^{\infty} f_{k}\left(\frac{\gamma m}{\bar{\gamma}}\right)^{k+m}\right)^{L-1}
$$

where $f_{k}=\frac{(-1)^{k}}{k !(m+k)}$.

The functional series representation is used to rewrite the series in (2.22) with the help of $[80$, pp. $19,(0.314)]$, as

$$
\left(\sum_{k=0}^{\infty} f_{k}\left(\frac{\gamma m}{\bar{\gamma}}\right)^{k+m}\right)^{L-1}=\left(\frac{\gamma m}{\bar{\gamma}}\right)^{m(L-1)} \sum_{p=0}^{\infty} c_{p}\left(\frac{\gamma m}{\bar{\gamma}}\right)^{p}
$$

leading to the following PDF

$$
p(\gamma, m)=\frac{L e^{-\frac{m \gamma}{\bar{\gamma}}}}{(\Gamma[m])^{L} \gamma} \sum_{p=0}^{\infty} c_{p}\left(\frac{m \gamma}{\bar{\gamma}}\right)^{p+m L}
$$


where $c_{0}=m^{1-L}, c_{p}=\frac{m}{p} \sum_{k=1}^{p}(k L-p) f_{k} c_{p-k}$. Then the DFP of the selection-based DF relay $P_{\mathrm{DFP}, \mathrm{sc}}$ can be accomplished, by using $[80$, pp. $356,(3.351 .2)]$, as

$$
\begin{aligned}
P_{\mathrm{DFP}, \mathrm{SC}}\left(\gamma_{t h}, m\right) & =\int_{\gamma_{t h}}^{\infty} p_{\gamma}(\xi, m) d \xi \\
& =\frac{L \exp \left(-\frac{m \gamma_{t h}}{\bar{\gamma}}\right)}{(\Gamma[m])^{L}} \sum_{p=0}^{\infty} \sum_{k=0}^{p+m L-1} c_{p} \frac{(p+m L-1) !}{k !}\left(\frac{m \gamma_{t h}}{\bar{\gamma}}\right)^{k} .
\end{aligned}
$$

The infinite summation in the PDF in (2.22) can be eliminated provided that that the integer values of $m$ are considered. This makes further simplification possible, leading to an alternative representation of this $\mathrm{PDF}[82]$ :

$$
p(\gamma, m)=\frac{L}{(m-1) !} \sum_{l=0}^{L-1}(-1)^{l}\left(\begin{array}{c}
L-1 \\
l
\end{array}\right) \sum_{k=0}^{l(m-1)} b_{k}^{l}\left(\frac{m}{\bar{\gamma}}\right)^{m+k} \gamma^{m+k-1} \exp \left(-(l+1) \frac{m \gamma}{\bar{\gamma}}\right)
$$

where $b_{0}^{l}=1, b_{1}^{l}=l, b_{l(m-1)}^{l}=\frac{1}{((m-1) !)^{l}}$, and $b_{k}^{l}=\frac{1}{k} \sum_{j=1}^{\min [k, m-1]} \frac{j(l+1)-k}{j !} b_{k-j}^{l}$ are recursively computed with $k=2,3, \cdots, l(m-1)-1$. With this PDF, a more convenient DFP expression for the selection-based DF relay can be obtained as

$$
\begin{aligned}
P_{\mathrm{DFP}, \mathrm{SC}}\left(\gamma_{t h}, m\right) & =\int_{\gamma_{t h}}^{\infty} p_{\gamma}(\xi, m) d \xi \\
& =\frac{L}{(m-1) !} \sum_{l=0}^{L-1}(-1)^{l}\left(\begin{array}{c}
L-1 \\
l
\end{array}\right) \sum_{k=0}^{l(m-1)} b_{k}^{l} \exp \left(-\frac{\gamma_{t h}(l+1) m}{\bar{\gamma}}\right) \\
& \times \sum_{t=0}^{m+k-1} \frac{(m+k-1) !}{t !} \frac{\left(m \gamma_{t h} / \bar{\gamma}\right)^{t}}{(l+1)^{m+k-t}} .
\end{aligned}
$$

Furthermore, the DFP of MRC-based relay detection which employs threshold decoding is investigated. In the case of MRC, since it is assumed that the branch fading amplitudes are statistically independent and distributed with Nakagami distribution, then, $\gamma$ also follows the Nakagami distribution but with a parameter $L m$ [83], leading to the following PDF

$$
p(\gamma, m)=\left(\frac{m}{\bar{\gamma}}\right)^{L m} \frac{\gamma^{L m-1}}{\Gamma[L m]} \exp \left(-\frac{m \gamma}{\bar{\gamma}}\right)
$$


Hence, the DFP of MRC-based relay detection can be obtained as

$$
P_{\mathrm{DFP}, \mathrm{MRC}}\left(\gamma_{t h}, m\right)=\frac{\exp \left(-\frac{m \gamma_{t h}}{\bar{\gamma}}\right)}{\Gamma[L m]} \sum_{k=0}^{L m-1} \frac{(L m-1) !}{k !}\left(\frac{m \gamma_{t h}}{\bar{\gamma}}\right)^{k} .
$$

Figure 2.10 shows the improvement in the probability of decoding at the relay when multiple antennas are deployed but one branch is selected. The plots give the DFP curves for $L=1,2,3,4$ and different Nakagami parameter $m$.

Figure 2.11 shows the impact of MRC detection on the DFP of a relay that has two antennas $(L=2)$. The figure gives curves for different Nakagami parameters $m=1,2,4$, and 6. The single antenna case is also reproduced in this figure as a base-line. Thus, a significant increase in the number of times the relay decodes and forwards is observed for the dual-antenna which implies that there is an increase in the number of times the destination relies on diversity combining using signals received via the relays. This, however, has to be complemented by the improved error performance at the relays (provided in Chapter 4). These relay performance indicators (relay error rate and probability of decode and forward) are shown to characterize the system end-to-end (E2E) performance.

Some final remarks on the derived expressions include the following. These expressions are valid for only integer values of $m$. It should be noted that the term in brackets in (2.22) is the incomplete gamma function in (2.19). Therefore, (2.22) could have been left with the incomplete gamma function and there will be a PDF that is valid for all possible values of $m$. The disadvantage of this approach is that no close-form representation may be pursued afterwards. The derivation presented above is valid for integer values of $m$ but it is not necessary to compute it numerically which would have been the case if there had not been efforts to simplify $(2.22)$. 


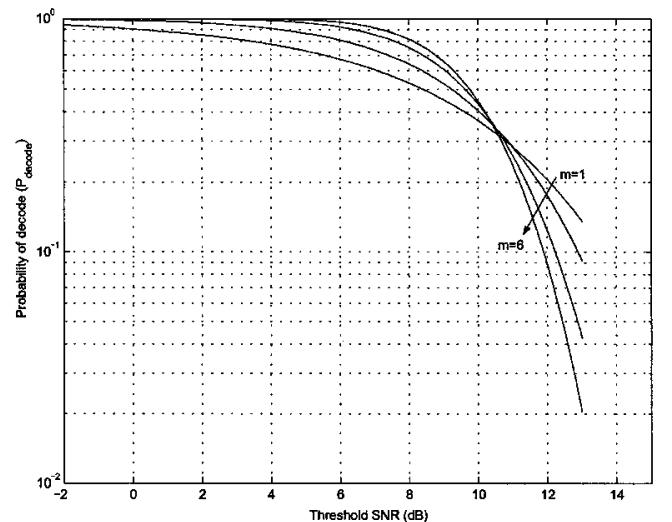

(a) $L=1$

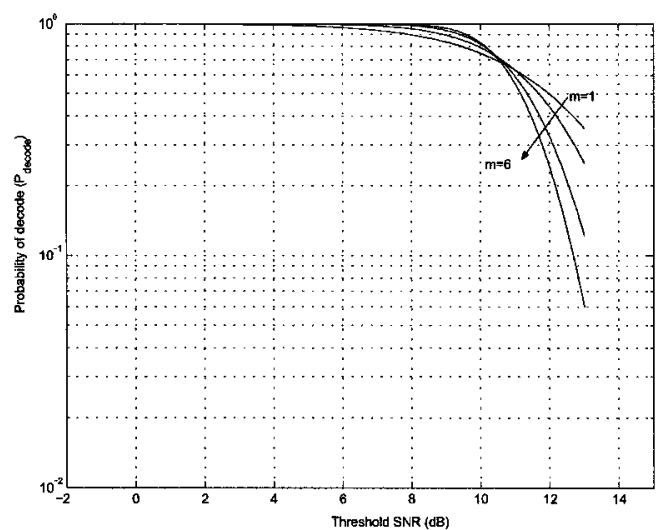

(c) $L=3$

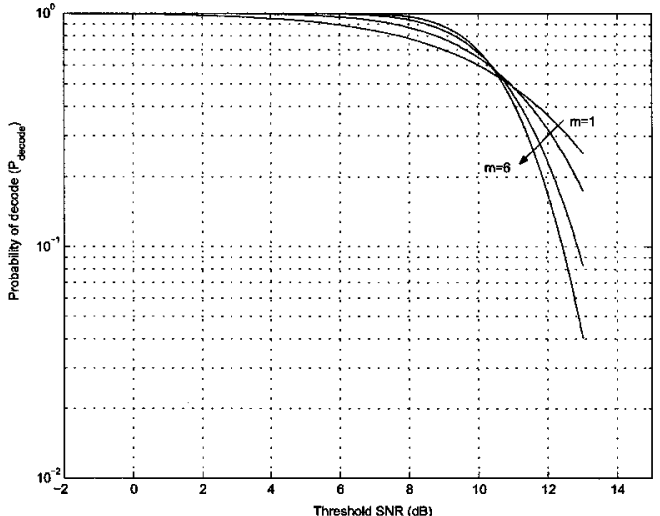

(b) $L=2$

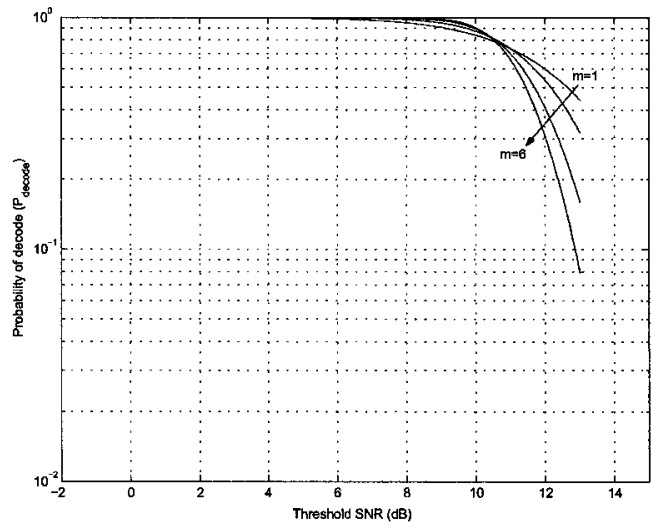

(d) $L=4$

Figure 2.10: The probability of decode of SC-based threshold decode-and-forward relay with different number of antennas $(L)$ for $m=1,2,4,6$, average SNR $=10 \mathrm{~dB}$. 


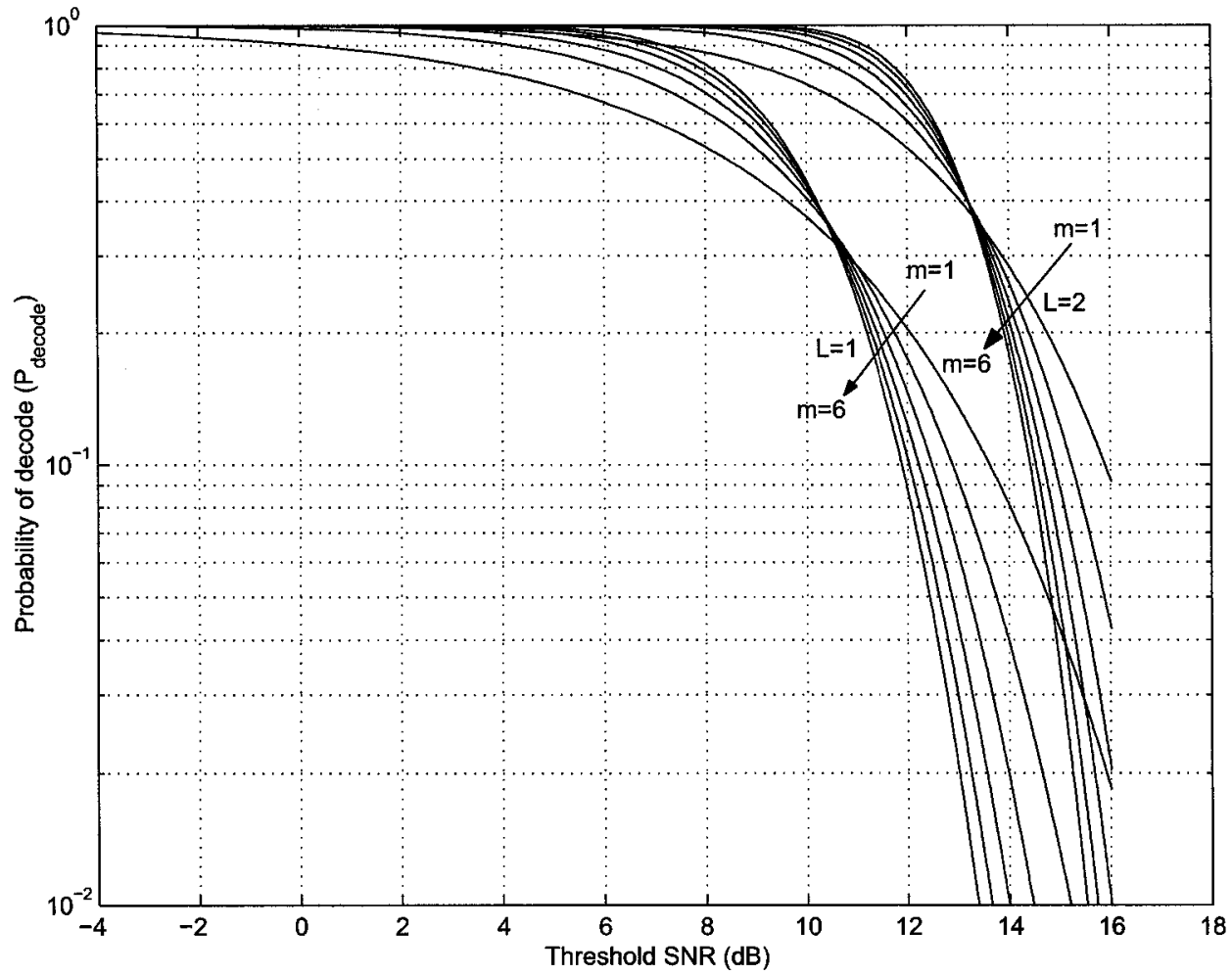

Figure 2.11: Probability of decode in MRC-based threshold-based decode-and-forward protocol, average SNR $=10 \mathrm{~dB}$. 


\subsection{Chapter Conclusion}

In this chapter a brief overview of wireless communications, challenges and techniques are presented. The potentials of multi-antenna and diversity combining, and relaying techniques for improving the performance of wireless systems so that they can support high data rate transmissions are discussed. Such performance enhancement from multi-antenna techniques is achieved without an increase in bandwidth. However, the constantly decreasing and miniaturized terminals pose obstacles to exploiting the benefits of multi-antenna techniques and hence, the use of cooperative relaying schemes discussed in subsequent chapters. In addition, an overview of the Nakagami fading model used in the thesis is presented. The impact of multiple antenna on the decode-and-forward probability of multi-antenna fixed relays is analyzed.

Nakagami model is adopted for the following reasons. In system deployment involving infrastructure-based fixed relays, the system engineer would try to locate the relays such that it has a line of site (LOS) to the base station. Most previous works on relaying have used a Rayleigh fading model that is not suitable in such LOS scenario. Our analysis therefore becomes handy in a wide range of channel scenarios, knowing that with proper choice of the Nakagami $m$ parameter, LOS scenarios can be modelled. Moreover, the Rayleigh fading model is a special case of the analysis. 


\section{Chapter 3}

\section{Hybrid Macro- and Micro-diversity Antenna Networks}

Microdiversity, through collocated antennas, has been used to combat small-scale fading. However, shadowing, which belongs to the large-scale fading class, could overwhelm a system with such closely spaced antennas. Emerging distributed and sectorized distributed antennas (SDA) $[15,16,17]$ and radio-on-fiber (RoF) technologies $[19,60]$ are providing novel ways of handling signals received at widely separated antennas. These technologies are particularly suitable for microcellular systems and they could facilitate linking of distributed antennas or access ports to a central unit where signal-specific processing could then be performed.

The chapter examines a microdiversity-augmented macrodiversity architecture to combat these two fading phenomena (small-scale fading and shadowing) in one scheme. The proposed system works as follows. In the $K$-macro/ $L$-microdiversity antenna structure, the macrodiversity selects the best among $K$ ports, using certain criteria, and of the $L$ signals available at that port, $l_{\circ}$ strongest are selected for diversity combining. Selecting a subset of spatial diversity signals to combine is known as generalized selection combining (GSC) and it was first applied to small scale fading [24]. GSC is employed as the receiver processor for the microdiversity component since it represents a reasonable system complexity and cost compromise 
compared with a full blown MRC [26, 27].

The previous work $[66,69]$ conventional scheme uses the macroscopic component alone as the basis for port selection. This selection criterion has been adopted with the setup described above and the technique is referred to as Scheme I. Analytical performance expressions have been derived for this scheme. Probably, a decade ago, selection based on the slow large scale fading was the most practical strategy to adopt. The advancement in distributed antenna system technologies, $\operatorname{RoF}[19,60]$, and the availability of a fast scanning receiver for the GSC [27] would allow for the fast tracking of the composite fading which hitherto might not have been recognized when the work in $[69,71]$ were being developed. With these technological advances in mind, this thesis attempts to estimate the performance of the system that would adopt these new technologies by proposing and evaluating the performance of Scheme II and Scheme III.

With microcellular network mind-set, we consider a network where SDA and RoF technologies are employed. Therefore, it is possible to avoid cell-splitting often used for improving system performance in conventional cellular systems. Instead, cost-efficient radio access points are deployed and linked to a central unit (CU) using the DA and RoF technologies, thereby, eliminating the need for new base stations and the associated resources. For the proposed architecture, this chapter introduces a novel way of implementing the macro-selection referred to as Scheme II, it also demonstrates its superiority over the conventional macro-selection method (Scheme I). Furthermore, through simulations macroscopic MRC in microcellular systems is explored. This is referred to as Scheme III. Since this scheme processes across the macro-diversity ports it can be viewed as an extension of the CDMA cellular soft handover to non-CDMA systems.

In Section 3.1, the channel and system models are discussed whereas Section 3.2 presents the proposed schemes. In Section 3.3 the derivation of expression for evaluating the error performance of Scheme I and numerical results are discussed. Section 3.4 presents the outage 


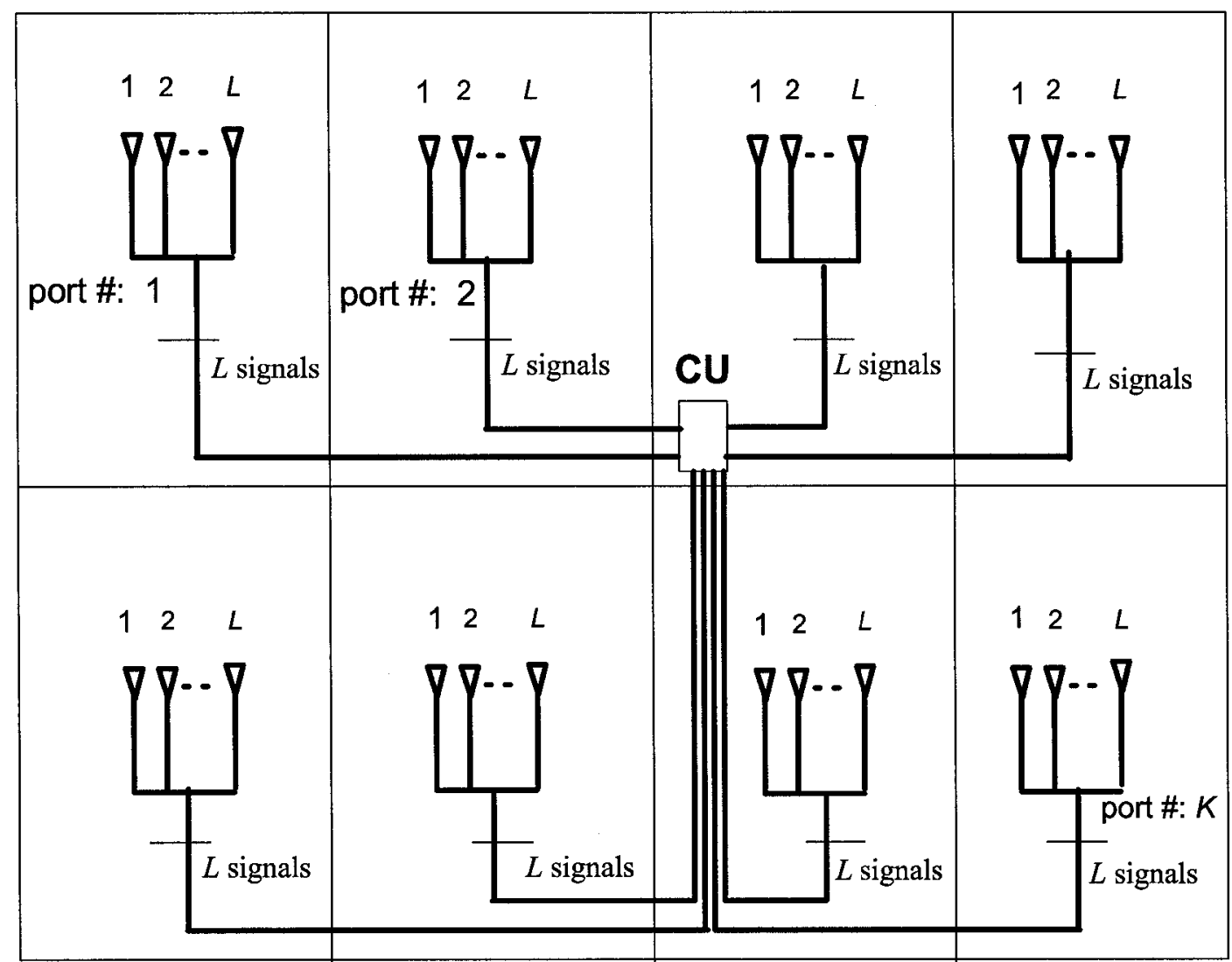

Figure 3.1: Antenna layout for the macro/microdiversity in a wireless network.

calculations and Section 3.5 discusses the impact of correlation while complexity issues are discussed in Section 3.6. Finally, conclusions are drawn in Section 3.7.

\subsection{System and Channel Descriptions}

Figure 3.1 shows the proposed distributed antenna architecture in a wireless network area. In the SDA format a separate feeder exists between each branch (microdiversity antenna) and the CU. Hence, a total of $K \times L$ distinct signals are received at the CU that enables the separation of the received signals. All the signal-specific processing (such as demodulation) is performed at the $\mathrm{CU}$; this results in relatively inexpensive ports. The channel between a port 
and a user is modeled as Rayleigh flat fading plagued with lognormal shadowing. The faded signal is perturbed by the receiver white Gaussian noise (AWGN), which is independent of the fading processes.

Let us consider that the signal $y_{k, l}$ is received at the $l$-th branch of the $k$-th port in the $K$-macro $/ L$-microdiversity architecture. This received signal can be expressed as

$$
y_{k, l}=\sqrt{\Lambda_{k}} \widetilde{\alpha}_{k, l} x+n_{k, l}, \quad 1 \leq l \leq L
$$

where $\mathbf{y}_{k}=\left\{y_{k, 1}, y_{k, 2}, \cdots, y_{k, L}\right\}$ is the set of received signals at port $k, x$ is the transmitted signal, and $\mathbf{n}_{k}=\left\{n_{k, 1}, n_{k, 2}, \cdots, n_{k, L}\right\}$ are the 2-dimensional AWGN. The $\Lambda$ represents the shadowing component. Let the branch gains $\sqrt{\Lambda_{k}} \widetilde{\alpha}_{k, l}$ be denoted as $\alpha_{k, l}$, where $k \epsilon\{1,2, \cdots, K\}$ and $l \epsilon\{1,2, \cdots, L\}$. These gains represent the composite fading with a common local mean square value $E\left[\alpha_{k, l}^{2}\right]=\Omega_{k}$. Therefore, at port $k$ the local mean received signal-to-noise ratio (SNR) per branch is $\frac{E_{s}}{N_{0}} \Omega_{k}$, where $E_{s}$ is the signal energy and $N_{0}$ is the single-sided power spectral density of the AWGN. The instantaneous SNR at each branch $\mathcal{S}_{k, l}=\alpha_{k, l}^{2} \frac{E_{s}}{N_{0}}$. The fact that $\Lambda_{k}$ is a lognormal random variable, implies that $G_{k}=10 \log _{10}\left(\Lambda_{k}\right)$ has a Gaussian distribution with a certain area mean $\mu_{k} \mathrm{~dB}$ and standard deviation $\sigma_{k} \mathrm{~dB}$.

\subsection{Macrodiversity Selection Schemes}

The macrodiversity selections methods are presented next, starting with the conventional method.

\subsubsection{Scheme I}

The local mean SNR is the basis of port selection. This scheme can therefore be referred to as selection combining-based (SC-based) macrodiversity

- Obtain the local means $\Omega_{k}, 1 \leq k \leq K$, noting that shadowing is same for all branches in a port. 
- Identify the port $k^{*}$ with the largest local mean; i.e., $k^{*}=\underbrace{\operatorname{argmax}}_{1 \leq k \leq K}\left\{\Omega_{k}\right\}$.

- Select $l_{\mathrm{o}} \leq L$ branches of this port $k^{*}$ with the largest $\mathcal{S}_{k^{*}, l}$.

Based on microcellular architecture and DAS, the following strategies can be adopted to take advantage of all the distinct $K \times L$ signals at the CU.

\subsubsection{Scheme II}

At each branch of a port, the SNR depends on both the macroscopic and microscopic fading, we can exploit this fact in making an informed port selection decision rather than deciding based only on the local mean SNR.

- Choose the port $k^{*}$ that has the largest overall aggregate SNR; i.e.,

$$
k^{*}=\underbrace{\operatorname{argmax}}_{1 \leq k \leq K}\left\{\sum_{l=1}^{L} \mathcal{S}_{k, l}\right\} .
$$

- Select $l_{\mathrm{o}} \leq L$ branches of this port $k^{*}$ with the largest $\mathcal{S}_{k^{*}, l}$.

In a suboptimal implementation of Scheme II, the port selection can be performed on the highest branch SNR;

- Identify $k^{*}=\underbrace{\operatorname{argmax}}_{1 \leq k \leq K}\{\underbrace{\max }_{1 \leq l \leq L} \mathcal{S}_{k, l}\}$.

- Select $l_{\circ} \leq L$ branches of this port $k^{*}$ with the largest $\mathcal{S}_{k^{*}, l}$.

A slightly more complex implementation of Scheme II which can improve the system performance is;

- Arrange each port $L$ signals in descending order.

- Choose the port $k^{*}$ that has the largest sum of $l_{\mathrm{o}}$ branch SNRs; i.e., $k^{*}=\underbrace{\operatorname{argmax}}_{1 \leq k \leq K}\left\{\sum_{l=1}^{l_{o}} \mathcal{S}_{k, l}\right\}$. 
The third realization of Scheme II will give the best system performance as it also appears as the reasonable receiver option but in practice, implementation consideration may make the first and second algorithms more attractive.

\subsubsection{Scheme III}

This scheme, by the virtue of all $K \times L$ available at the CU, processes across the ports.

- Arrange all the $K \times L$ signals in ascending order of their SNR, $\mathcal{S}_{k, l}$, across all ports.

- Select the largest $l_{\mathrm{o}}$ out of the $K \times L$ branches.

In all cases, MRC technique is used to process the $l_{\text {o }}$ selected branches, where $1 \leq l_{\circ} \leq L$.

\subsection{Performance Analysis}

In this section the analysis of GSC-augmented macrodiversity (Scheme I) is performed. Let $\beta, l_{o}$, and $\Lambda^{*}$ represent the sum of the combined Rayleigh fading branches at the selected port, the number of microdiversity branches selected, and the shadowing component at the selected port, respectively. Note that $\beta=\sum_{i=1}^{l_{o}} \widetilde{\alpha}_{k^{*}, i}^{2}$ and $\Lambda^{*}=10^{g^{*} / 10}$. The symbol error probability (SEP) for MPSK modulation conditioned on the fade samples can be expressed for GSC as

$$
P_{\mathrm{mpsk}}\left(e \mid \beta, g^{*}\right) \approx h \times \operatorname{erfc}\left(\sqrt{\beta \Lambda^{*} \sin ^{2}\left(\frac{\pi}{M}\right) \frac{E_{s}}{N_{0}}}\right),
$$

MPSK has been used as an illustrative example, other coherent modulation schemes having error rate defined by complementary error function erfc (or Q function) in AWGN can also be accommodated in the analysis presented here.

Equation (3.2) implies that the error performance is conditioned on the parameters $\beta$ and $g^{*}$ and therefore, to obtain the error performance, we need to average over two PDFs as 
follows:

$$
\begin{aligned}
P_{\mathrm{mpsk}}(e) & =\int_{0}^{\infty} \int_{-\infty}^{\infty} P_{\mathrm{mpsk}}\left(e \mid \beta, g^{*}\right) p\left(\beta ; l_{\mathrm{o}}\right) p_{G^{*}}\left(g^{*} ; K\right) d g^{*} d \beta \\
& =\int_{-\infty}^{\infty} p_{G^{*}}\left(g^{*} ; K\right) \underbrace{\int_{0}^{\infty} p\left(\beta ; l_{\mathrm{o}}\right) P_{\mathrm{mpsk}}\left(e \mid \beta, g^{*}\right) d \beta}_{{ }_{\mathrm{mpsk}}\left(e \mid g^{*}\right)} d g^{*}
\end{aligned}
$$

The PDF of $\beta$ can be expressed, by the help of the results presented in [26], as

$$
p\left(\beta ; l_{\mathrm{o}}\right)=C_{l_{\mathrm{o}}}^{L}\left[\frac{\beta^{l_{\mathrm{o}}-1} e^{-\beta}}{\left(l_{\mathrm{o}}-1\right) !}+\sum_{l=1}^{L-l_{\mathrm{o}}} C_{l}^{L-l_{\mathrm{o}}}(-1)^{l_{\mathrm{o}}+l-1}\left(\frac{l_{\mathrm{o}}}{l}\right)^{l_{\mathrm{o}}-1} e^{-\beta}\left(e^{-\frac{l \beta}{l_{\mathrm{o}}}}-\sum_{p=0}^{l_{\mathrm{o}}-2} \frac{1}{p !}\left(-\frac{l \beta}{l_{\mathrm{o}}}\right)^{p}\right)\right],
$$

where $C_{r}^{R}=\frac{R !}{(R-r) ! r !}$. Using this PDF in (3.3), and with some manipulations, the expression for the SEP of MPSK conditioned on the lognormal shadowing can be expressed as ${ }^{1}$

$$
P_{\mathrm{mpsk}}\left(e \mid g^{*}\right) \approx h C_{l_{\mathrm{o}}}^{L}\left[\begin{array}{l}
\frac{1}{2^{2 l_{\mathrm{o}}-2}} \sum_{j=1}^{l_{o}} C_{l_{\mathrm{o}}-1}^{2 l_{\mathrm{o}}-j-1}\left(\frac{2}{1+\sqrt{\theta_{1}}}\right)^{j} \frac{1}{\left(1+\gamma^{*} \lambda\right)^{l_{\mathrm{o}}}}+\sum_{l=1}^{L-l_{\mathrm{o}}} \frac{2 \eta\left(l, l_{\mathrm{o}}, L\right)}{\left(l / l_{\mathrm{o}}+1+\gamma^{*} \lambda\right)\left(1+\sqrt{\theta_{2}}\right)} \\
-\sum_{l=1}^{L-l_{\mathrm{o}}} \eta\left(l, l_{\mathrm{o}}, L\right) \sum_{p=0}^{l_{\mathrm{o}}-2} \frac{4\left(-l / l_{\mathrm{o}}\right)^{p}}{\left[4\left(1+\gamma^{*} \lambda\right)\right]^{p+1}} \sum_{j=1}^{p+1} C_{p}^{2(p+1)-j-1}\left(\frac{2}{1+\sqrt{\theta_{1}}}\right)^{j}
\end{array}\right],
$$

where, $\eta\left(l, l_{\mathrm{o}}, L\right)=C_{l}^{L-l_{\mathrm{o}}}(-1)^{l_{\mathrm{o}}+l-1}\left(l_{\mathrm{o}} / l\right)^{l_{\mathrm{o}}-1}, \theta_{1}=\frac{\Lambda^{*} \lambda}{1+\Lambda^{*} \lambda}, \theta_{2}=\frac{\Lambda^{*} \lambda}{1+\Lambda^{*} \lambda+l / l_{\mathrm{o}}}, \Lambda^{*}=10^{g^{*} / 10}$ and $\lambda=q \sin ^{2}\left(\frac{\pi}{M}\right) \frac{E_{s}}{N_{0}}$. For BPSK $q=1$ and $h=1 / 2$ and equality is satisfied in (3.5)

In a situation where Scheme $\mathrm{I}$ is adopted, then $G^{*}=\max \left\{G_{1}, G_{2}, \cdots, G_{K}\right\}$ is the local mean value which corresponds to the port selected for the macroscopic diversity. For identically and independently distribution, the probability density function (PDF) of $G^{*}$ can be written [69] as

$$
p_{G^{*}}\left(g^{*} ; K\right)=\frac{K}{\sqrt{2 \pi \sigma^{2}}} \exp \left(-\frac{\left(g^{*}-\mu\right)^{2}}{2 \sigma^{2}}\right)\left[1-\frac{1}{2} \operatorname{erfc}\left(\frac{g^{*}-\mu}{\sqrt{2 \sigma^{2}}}\right)\right]^{K-1} .
$$

Finally, the error probability can be obtained by evaluating the integral in (3.3), that is,

$$
P_{\mathrm{mpsk}}(e)=\int_{-\infty}^{\infty} P_{\mathrm{mpsk}}\left(e \mid g^{*}\right) p_{G^{*}}\left(g^{*} ; K\right) d g^{*} .
$$

The integral in (3.7) can easily be evaluated using numerical integration techniques available in most standard mathematical software packages. However, evaluating it in a closed form remains an open problem.

\footnotetext{
${ }^{1}$ See Appendix C.3 for the derivation of $P_{\mathrm{mpsk}}\left(e \mid g^{*}\right)$.
} 


\subsubsection{Numerical Results}

Numerical results are presented for the GSC-augmented macroscopic diversity using the expressions derived for Scheme I. However, simulation results are presented for Schemes II and III, since deriving their performance expressions is more demanding. Different values for the standard deviation of the lognormal shadowing, which spans a wide range of wireless applications, are considered. For instance, for macrocellular applications $\sigma$ ranges from 5 to $12 \mathrm{~dB}$ with $\sigma=8 \mathrm{~dB}$ being a typical value whereas for microcells it varies between 4 and $13 \mathrm{~dB}$ [37].

Figure 3.2 shows the bit error probability (BER) versus SNR per branch for BPSK modulation scheme employed with this hybrid scheme for an antenna configuration of $K=3, L=5$, $1 \leq l_{\mathrm{o}} \leq L$, under the assumption of uncorrelated lognormal shadowing. The scenario where no microdiversity is employed $\left(K=3, L=1, l_{\circ}=1\right)$, and that where neither macrodiversity nor microdiversity is used $\left(K=1, L=1, l_{\mathrm{o}}=1\right)$ are also shown for comparison purposes. The variance of the lognormal shadowing $\sigma$ is set to $12 \mathrm{~dB}$. The simulated results are denoted by $\left({ }^{*}\right)$, it is observed that they agree well with the analytical ones. Figure 3.3 shows the performance for BPSK modulation with antenna configuration $K=2, L=5,1 \leq l_{\circ} \leq L$; in this case, $\sigma$ is set to $8 \mathrm{~dB}$. The simulation results are also plotted and denoted by $\left({ }^{*}\right)$, which match well with the derived expression. The case of no microdiversity and the case of no macrodiversity/no microdiversity are also given.

The performance superiority of the hybrid schemes over these two reference scenarios are clearly visible in Figures 3.2 and 3.3, and the significant gain of the hybrid schemes over the channel employing no form of diversity is clearly evident. It is interesting to observe from Figures 3.2 and 3.3 that there seems to be a fixed gain in SNR of about $2 \mathrm{~dB}$ for the system when $l_{o}$ is increased from one to two, for all $K$ and $L$ values considered. This $2 \mathrm{~dB}$ gain does not seem to be affected by the amount of lognormal shadowing. However, there

are diminishing returns as $l_{\mathrm{o}}$ continues to increase. In these figures, a $1 \mathrm{~dB}$ gain is generally observed when $l_{\mathrm{o}}$ is increased from 2 to 3 . The same trend is observed for the other $K$ and $L$ 


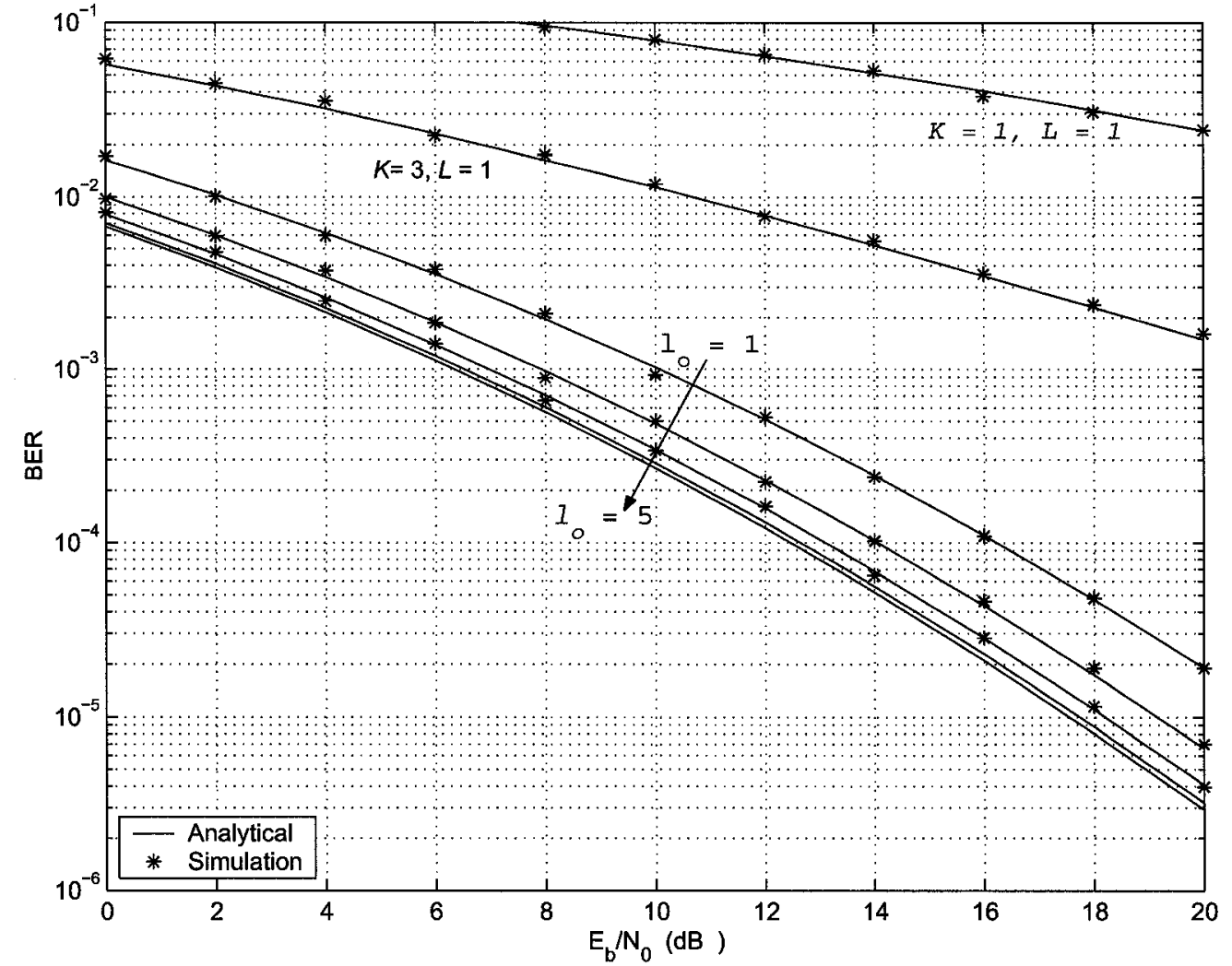

Figure 3.2: Performance of BPSK in conventional macrodiversity and GSC-based microdiversity (Scheme I) with lognormal shadowing $(\sigma=12 \mathrm{~dB})$ and Rayleigh fading; $K=3, L=$ $5,1 \leq l_{\circ} \leq L$ 


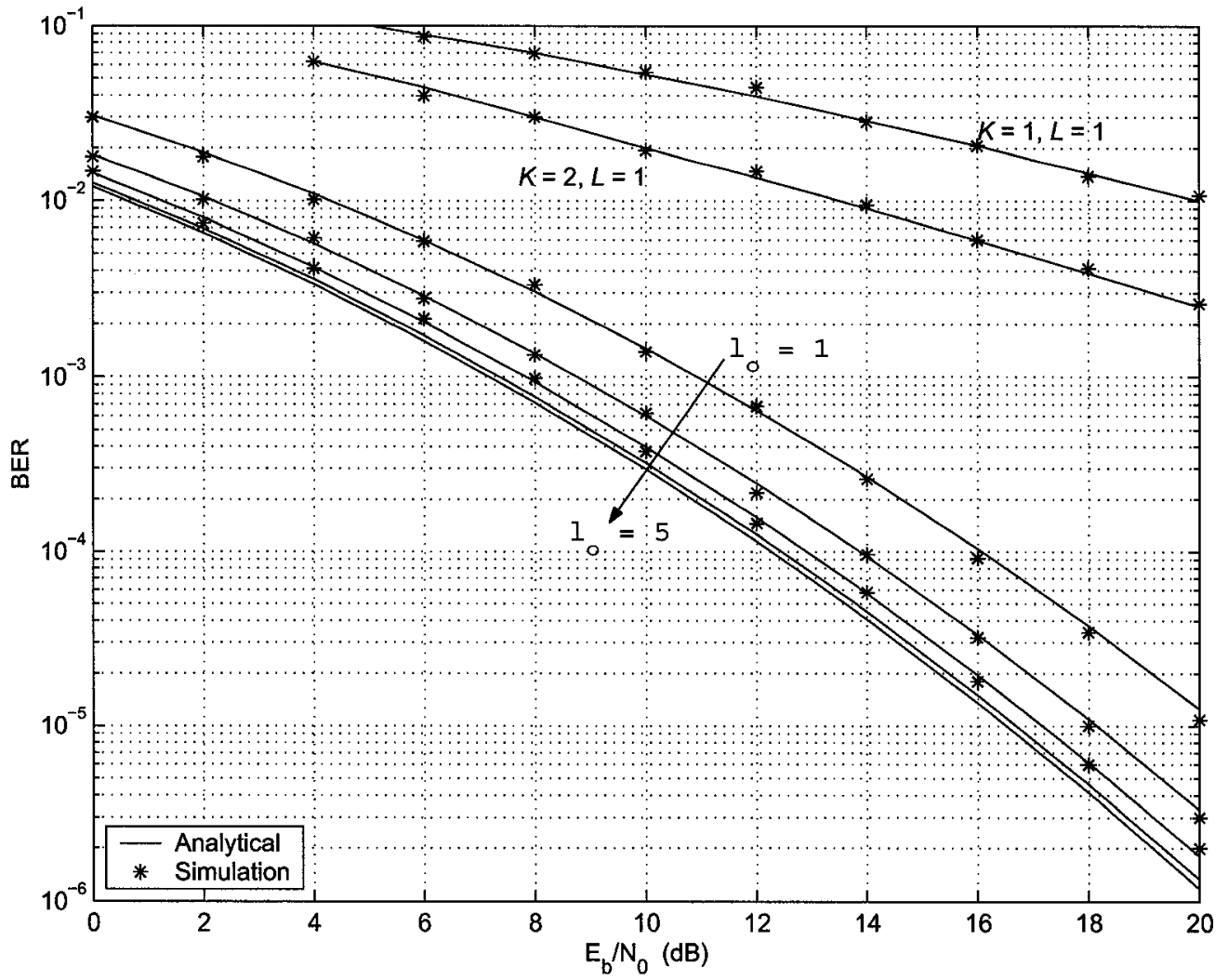

Figure 3.3: Performance of BPSK in conventional macrodiversity and GSC-based microdiversity (Scheme I) with lognormal shadowing $(\sigma=8 \mathrm{~dB})$ and Rayleigh fading; $K=2, L=5,1 \leq$ $l_{\mathrm{o}} \leq L$. 


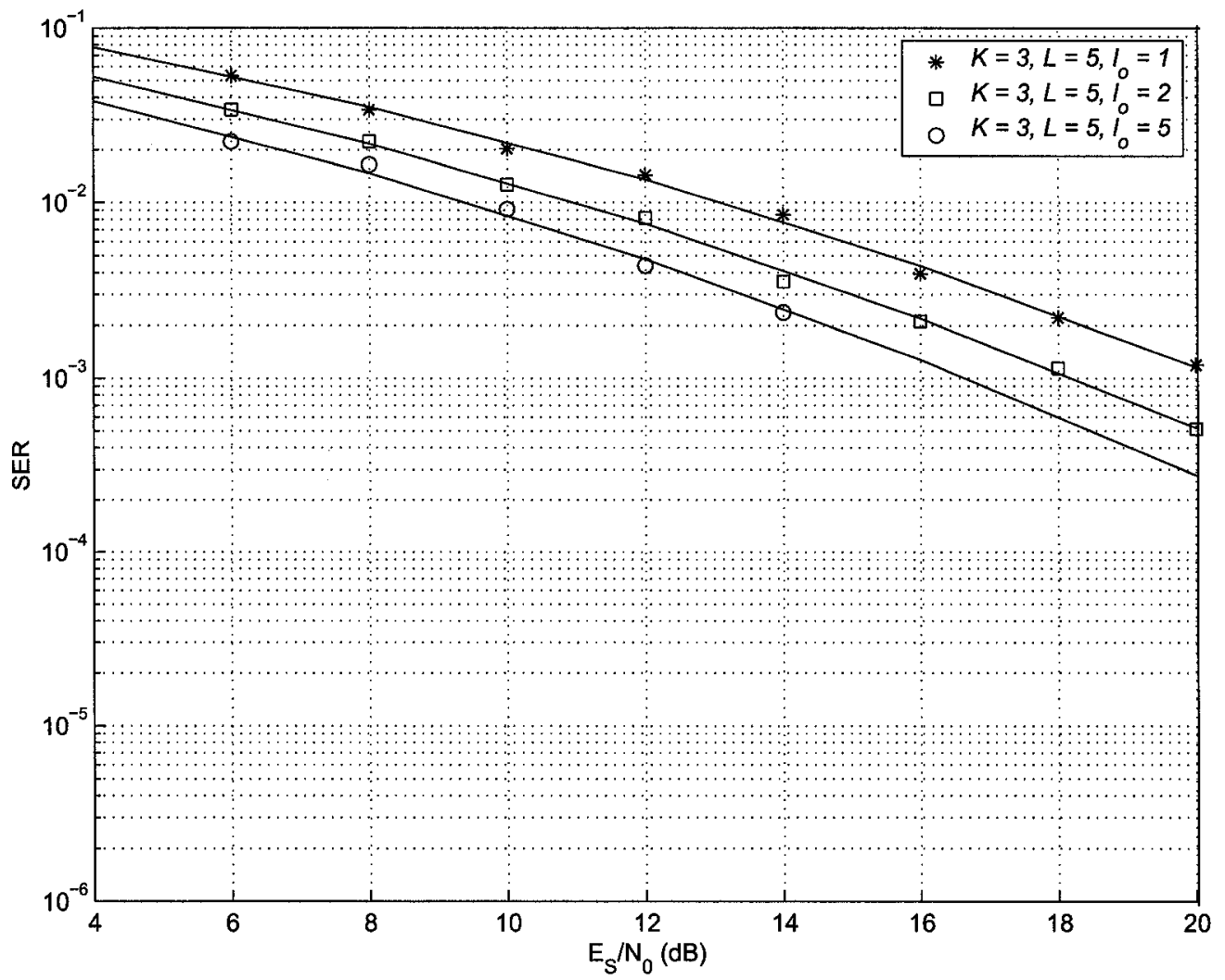

Figure 3.4: Performance of 8-PSK in conventional macrodiversity and GSC-based microdiversity (Scheme I) with lognormal shadowing $(\sigma=12 \mathrm{~dB})$ and Rayleigh fading.

combinations we have examined. In all cases, simulations are also conducted to validate the analytical results. We also observe that when $L=5$, for example, $l_{\mathrm{o}}=4$ has already given almost all the diversity benefit.

Figure 3.4 shows the performance of 8-PSK with shadowing $(\sigma=12 \mathrm{~dB})$ and Rayleigh fading for the antenna combination $K=3, L=5, l_{\mathrm{o}}=1,2,5$. The simulation results (denoted with symbols) confirm the accuracy of our analytical results for the higher constellation MPSK. Results for other higher constellations (e.g., 16-PSK) and different antenna configurations have exhibited the same trend of agreement between the simulations and analysis

The algorithms proposed and described as Scheme II (the first one) and Scheme III have 
been used to evaluate the probability of error for different combinations of $K, L$, and $l_{\mathrm{o}}$ for BPSK modulation. The performance of these algorithms is also compared with its conventional counterpart, i.e., Scheme I. In Figure 3.5, three set of curves are shown for the following values of diversity parameters, $K=2, L=5, l_{\mathrm{o}}=1 ; K=2, L=5, l_{\mathrm{o}}=2$; and $K=3, L=5, l_{\mathrm{o}}=3$. The value of $\sigma$ used is $8 \mathrm{~dB}$. It is observed that for $K=3, L=5, l_{\mathrm{o}}=3$, Scheme III has about $1.5 \mathrm{~dB}$ advantage over the conventional Scheme I at BER $=10^{-4}$. Considering the case $K=2, L=5, l_{\mathrm{o}}=3$, the novel schemes (Schemes II and III) still outperform the conventional Scheme I, but with reduced gain margins. At lower error rates, the proposed schemes enjoy more gains over the conventional scheme. For instance, for Scheme III these gains are close to $3 \mathrm{~dB}$ at $\mathrm{BER}=10^{-5}$ for $K=3, L=5, l_{\mathrm{o}}=3$.

The results obtained can be explained in the following way. Scheme III outperforms Scheme II which, in turns, performs better than Scheme I. Scheme III can be seen as a benchmark (or performance lower bound) of the hybrid schemes. In Scheme III the best $l_{\mathrm{o}}$ branches will always be selected. However, in Scheme II, it can not always be guaranteed that this will be the case, leading to the inferior performance of Scheme II as compared to that of Scheme III.

The performance improvement of Scheme II over Scheme I comes from just the way the received signals are treated without the need for extra ordinary signal processing, demonstrating that with modest extra processing efforts (Section 3.6), an additional gain as high as $3 \mathrm{~dB}$ can be obtained in the proposed hybrid macro/microdiversity scheme.

\subsection{Outage Probability Calculation}

An outage is said to occur when a certain link quality metric (for example, bit error rate of a user) becomes worse than a required threshold value. We present a performance expression for calculating the probability that the instantaneous error rate exceeds a certain threshold for a stationary user in the microdiversity augmented macrodiversity architecture (Scheme I). A quasi-static outage performance is given, in the sense that the user will be assumed to be 


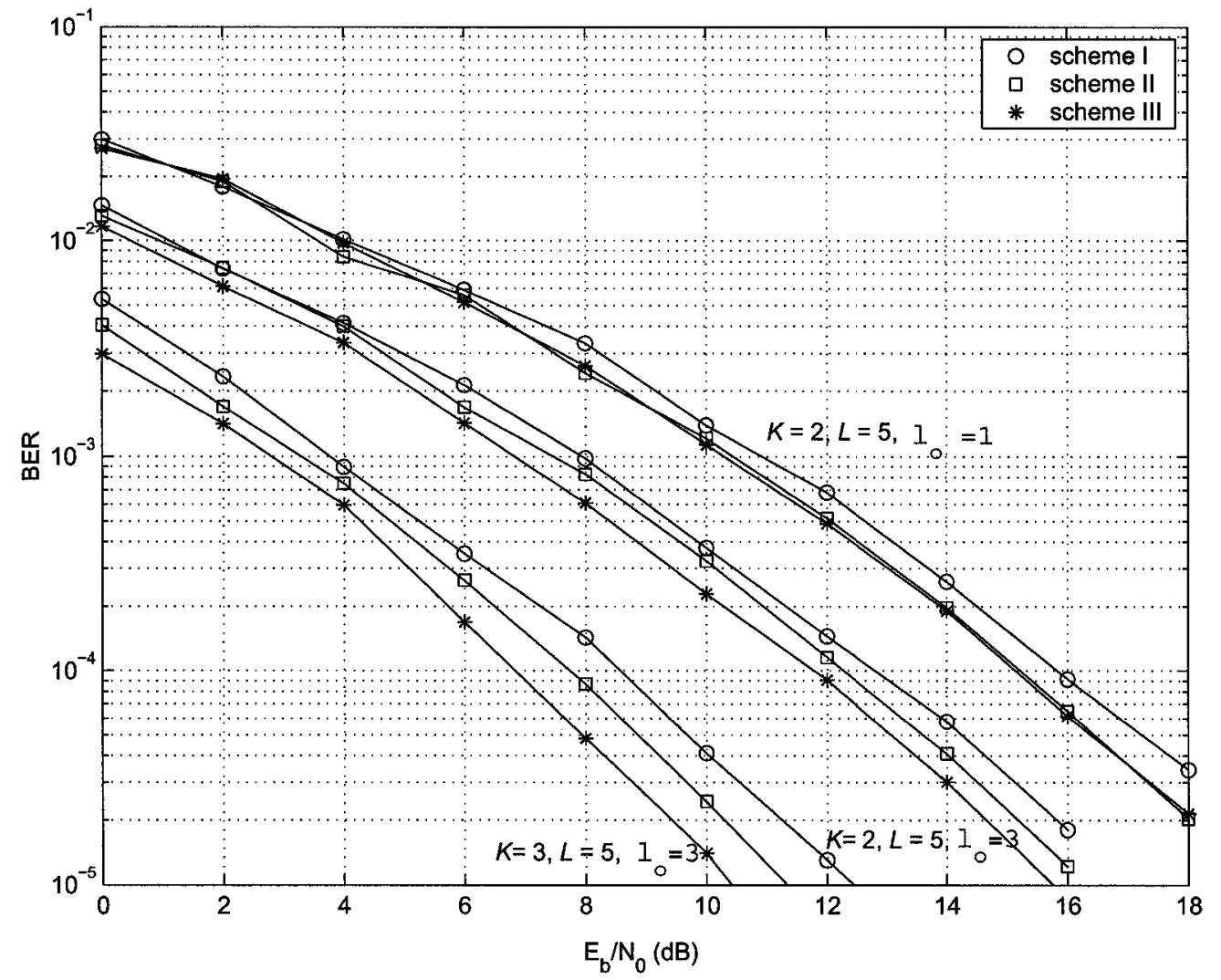

Figure 3.5: Performance comparison of the selection schemes with $\sigma=8 \mathrm{~dB}$ for different network configurations for BPSK. 
stationary at the point the average outage is evaluated, however, this analysis can easily be extended to mobile outage as well.

The instantaneous SNR can be expressed as $\beta \Lambda^{*} \frac{E_{b}}{N_{0}}$ and hence, the corresponding conditional bit error rate for $\mathrm{QPSK}^{2}, P_{\mathrm{qpsk}}\left(e \mid \beta, g^{*}\right)$ is

$$
P_{\mathrm{qpsk}}\left(e \mid \beta, g^{*}\right)=\frac{1}{2} \operatorname{erfc}\left(\sqrt{\beta \Lambda^{*} E_{b} / N_{0}}\right)
$$

Note that the probability of $P_{\mathrm{qpsk}}\left(e \mid \beta, g^{*}\right)$ being greater than a set threshold, say $\zeta$, is equivalent to the probability of having $\beta$ that is less than a threshold $\beta_{t h r}$ for a given $g^{*}$. This threshold value of $\beta$ can be expressed as

$$
\beta_{t h r}=\frac{\left[\operatorname{erfc}^{-1}(2 \zeta)\right]^{2}}{\Lambda^{*} \frac{E_{b}}{N_{0}}} .
$$

With this representation, the quasi-static outage probability can be obtained by averaging over the spatial environment

$$
\begin{aligned}
P_{\text {outage }} & =\int_{-\infty}^{\infty} \int_{0}^{\beta_{t h r}} p\left(\beta ; l_{o}\right) p_{G^{*}}\left(g^{*} ; K\right) d \beta d g^{*}, \\
& =\int_{-\infty}^{\infty} p_{G^{*}}\left(g^{*} ; K\right) \underbrace{\int_{0}^{\beta_{t h r}} p\left(\beta ; l_{o}\right) d \beta}_{I_{1}} d g^{*} .
\end{aligned}
$$

The integral denoted as $I_{1}$ can be expressed in the following form using the PDF given in

$$
\begin{aligned}
I_{1} & =C_{l_{\mathrm{o}}}^{L}\left[1-e^{-\beta_{t h r}} \sum_{k=0}^{l_{\mathrm{o}}-1} \frac{\beta_{t h r}^{k}}{k !}\right] \\
& +C_{l_{\mathrm{o}}}^{L}\left[\sum_{l=1}^{L-l_{\mathrm{o}}} \eta\left(l, l_{\mathrm{o}}, L\right)\left(\frac{1-e^{-\beta_{t h r}\left(\frac{l+l_{\mathrm{o}}}{l_{\mathrm{o}}}\right)}}{\frac{l_{\mathrm{o}}+l}{l_{\mathrm{o}}}}-\sum_{p=0}^{l_{\mathrm{o}}-2}\left(\frac{-l}{l_{\mathrm{o}}}\right)^{p}\left(1-e^{-\beta_{t h r}} \sum_{t=0}^{p} \frac{\beta_{t h r}^{t}}{t !}\right)\right)\right]
\end{aligned}
$$

Expressions (3.10) and (3.11) can be used to evaluate the average outage probability employing numerical methods.

Notice that the outage for other modulation schemes can similarly be obtained. The only effort required is to obtain an expression for the threshold on $\beta$; the direction of work being 


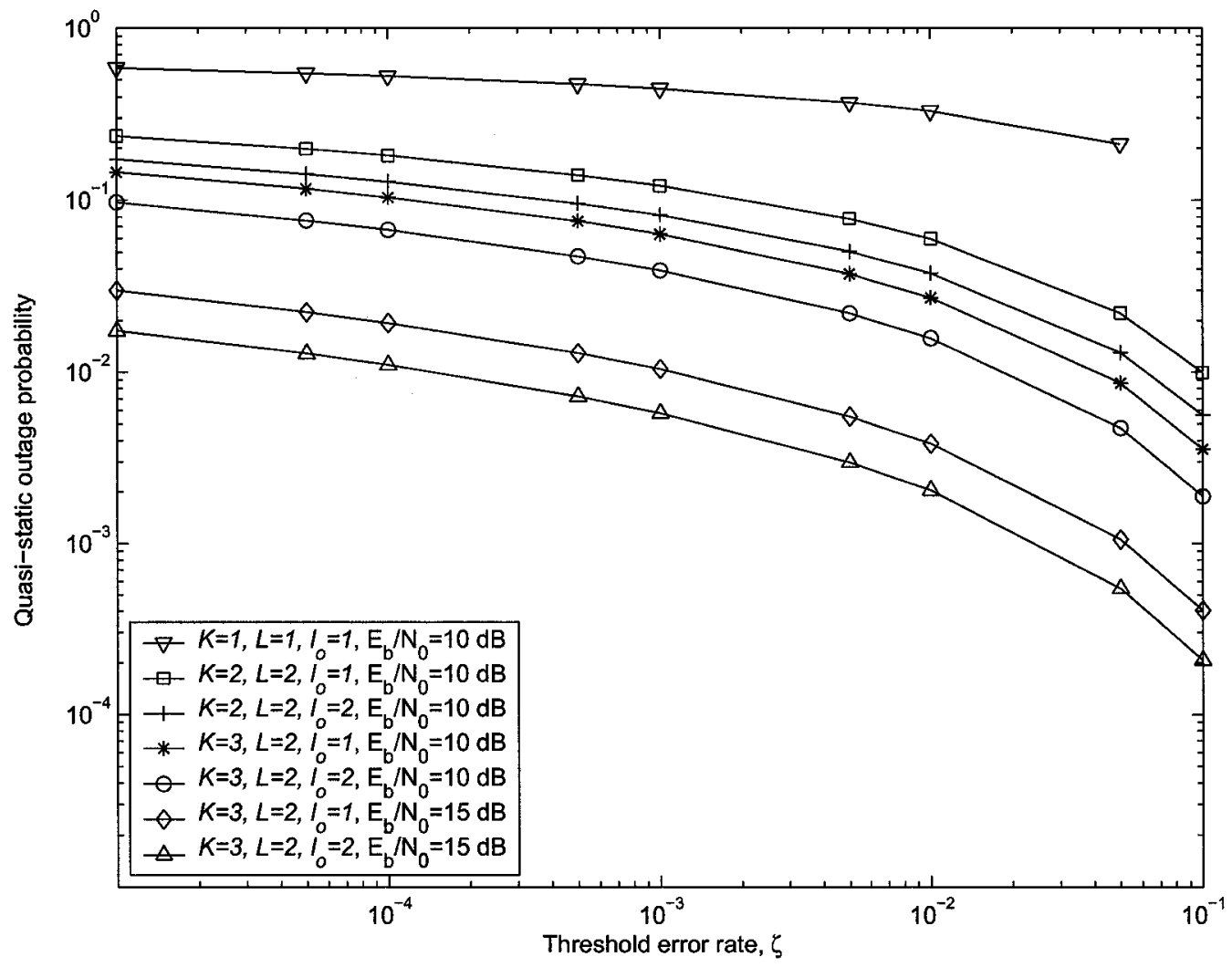

Figure 3.6: Outage probability of QPSK with Scheme I ( $\sigma=6 \mathrm{~dB})$. 
developed in [70] will be helpful in obtaining this threshold level. The curves in Figure 3.6 show the outage probability versus threshold bit error rate of QPSK. Different values of $K$, $L$, and $l_{\mathrm{o}}$, are used with different values of branch $E_{b} / N_{0}$ for $\sigma=6 \mathrm{~dB}$. It can be observed that to attain low error rates we require very large $E_{b} / N_{0}$ in channels subjected to fading and shadowing, and that the combination of microdiversity and macrodiversity reduces this power requirements. For the outage curves, it is observed that for a given error rate threshold, the amount of reduction in outage increases with the number of diversity branches. Furthermore, observe that there is a significant reduction in outage with $K=2, L=2, l_{\circ}=1$ over the case $K=1, L=1, l_{\mathrm{o}}=1$, although, at each processing instant, one detection resource is employed. In the following section, the impact of correlated shadowing at ports is discussed.

\subsection{Effects of Correlation on System Performance}

Studies have shown that the effects of correlation should not be ignored in claiming macrodiversity gains in the performance analysis of small cell systems [71, 72]. Numerical results are, therefore, presented to quantify the detrimental effects of correlation at the ports. The zero correlation case therefore, serves as a lower bound for the system performance.

Consider a 2-port case with correlated shadowing (measured through $\rho$ ). The PDF of the correlated shadowing can be expressed as [72]

$$
p_{G^{*}}\left(g^{*} ; 2\right)=\sqrt{\frac{2}{\pi \sigma^{2}}} \exp \left(-\frac{\left(g^{*}-\mu\right)^{2}}{2 \sigma^{2}}\right)\left[1-\frac{1}{2} \operatorname{erfc}\left(\sqrt{\frac{1-\rho}{1+\rho}}\left(\frac{g^{*}-\mu}{\sqrt{2 \sigma^{2}}}\right)\right)\right] .
$$

This PDF can be used in (3.7) to evaluate the effect of correlated shadowing on error performance.

The results presented in Figures 3.7 and 3.8 show the detrimental effect of correlated ports on Scheme I. For example, for a target error rate of $10^{-3}$ and correlation coefficient of $\rho=0.5$, between $4.5-6 \mathrm{~dB}$ additional SNR is required in comparison to no correlation

\footnotetext{
${ }^{2}$ Note that the BER of QPSK is equal to that of BPSK.
} 


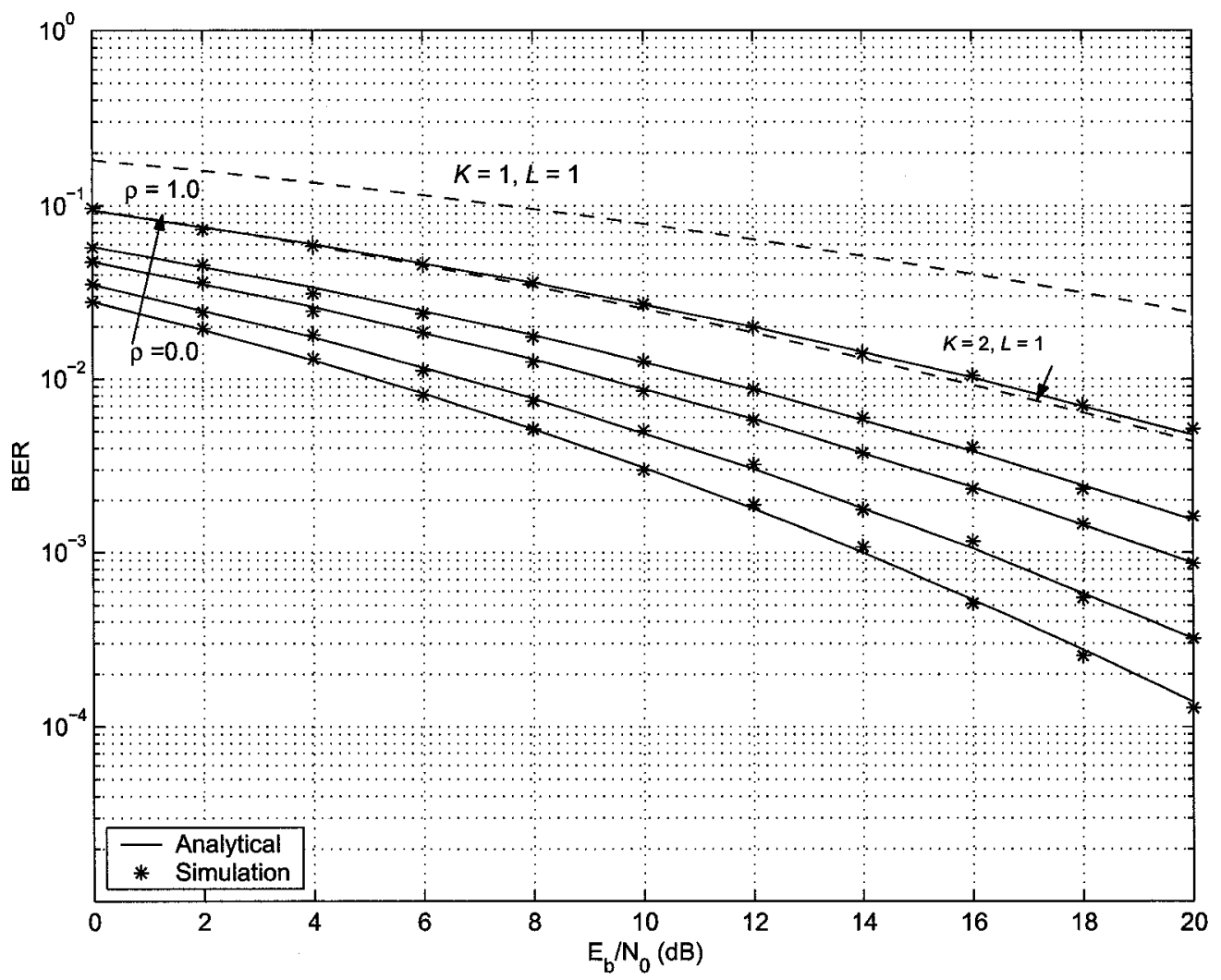

Figure 3.7: Performance of BPSK with Scheme I in correlated macrodiversity with $\sigma=12$ $\mathrm{dB} ; K=2, L=5, l_{\mathrm{o}}=2, \rho=0,0.2,0.5,0.7,1.0$. 


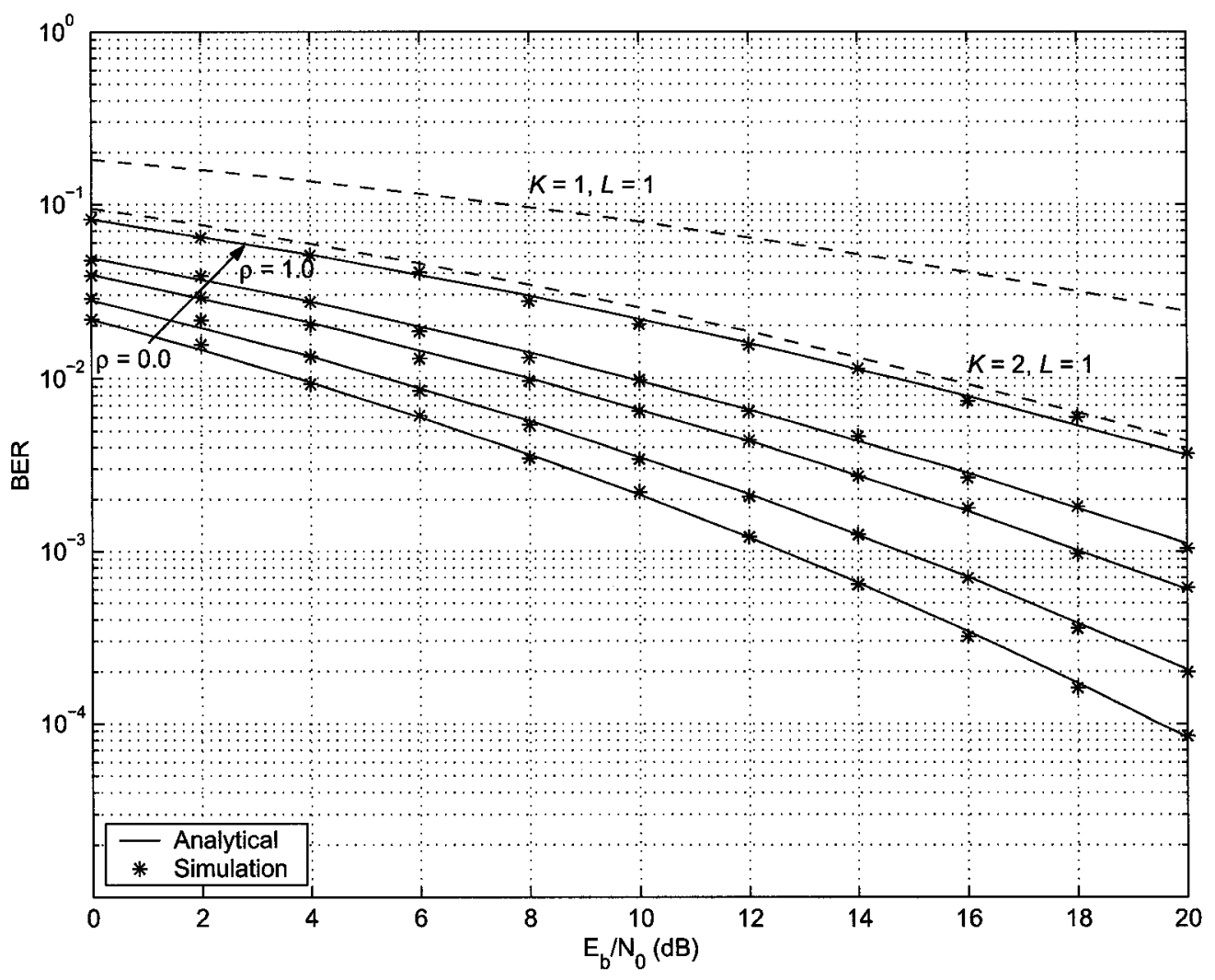

Figure 3.8: Performance of BPSK with Scheme I in correlated macrodiversity with $\sigma=12$ $\mathrm{dB} ; K=2, L=5, l_{\mathrm{o}}=4, \rho=0,0.2,0.5,0.7,1.0$. 


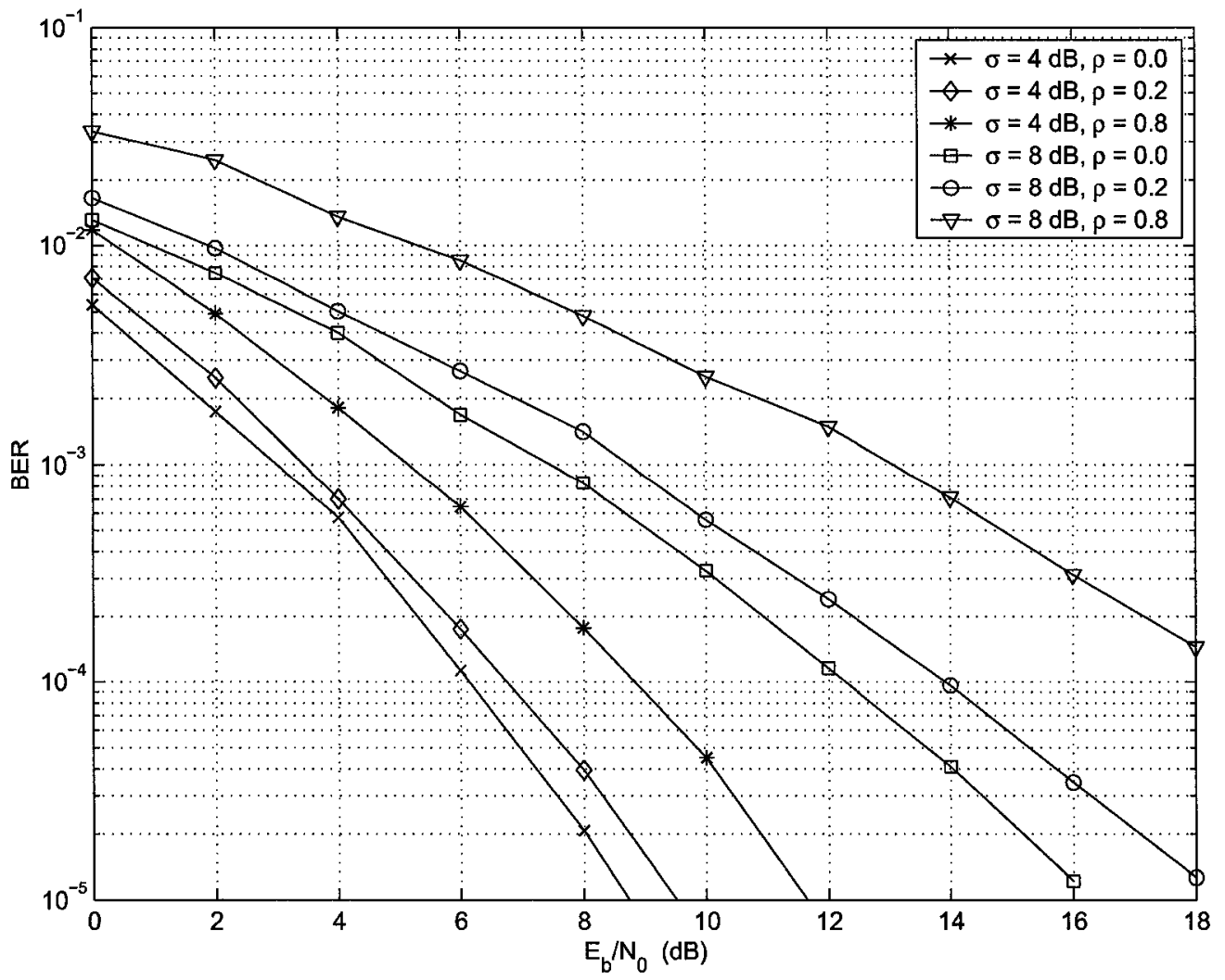

Figure 3.9: Effect of correlated shadowing on the performance of BPSK with Scheme II for two different $\sigma$ values, and $K=2, L=5, l_{\mathrm{o}}=3$.

for all of the $l_{\mathrm{o}}$ order microscopic combining cases examined. The simulation results are in agreement with those of analysis. The performance curves for the reference macrodiversity alone case $\left(K=2, L=1, l_{\mathrm{o}}=1\right)$ and the case with neither macrodiversity nor microdiversity $\left(K=1, L=1, l_{\circ}=1\right)$ are also shown. Comparing macrodiversity alone case with $(K=2, L=$ $5, l_{\mathrm{o}}=2$ and $l_{\mathrm{o}}=4$ ) and $\rho=1.0$ (completely correlated branches) reveals the tremendous loss in SNR due to the correlated shadowing, leading to the conclusion that in this scenario, increasing $L$ is not a reasonable solution. It can be observed in Figure 3.8 that for fully correlated ports, the configuration $K=2, L=5, l_{\mathrm{o}}=4$ is only a fraction of a dB superior to the $K=2, L=1, l_{\circ}=1$ configuration with no correlation. Figures 3.9 and 3.10 show the 


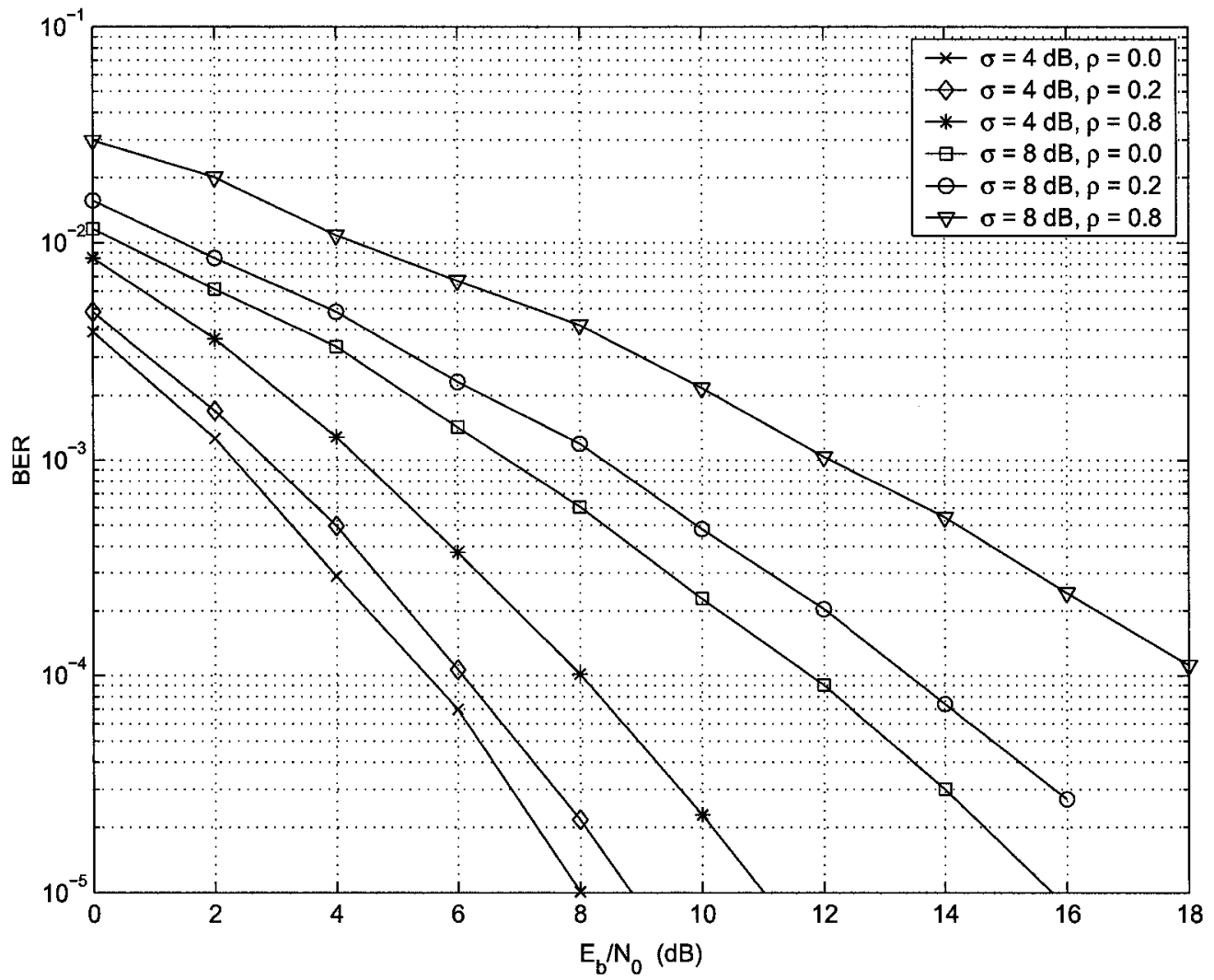

Figure 3.10: Effect of correlated shadowing on the performance of BPSK with Scheme III for two different $\sigma$ values; $K=2, L=5, l_{\mathrm{o}}=3$. 
impact of $\rho=0.2$ and $\rho=0.8$, referred to as moderate and significant correlation, respectively, on the proposed Schemes II and III. Two macrodiversity ports $(K=2)$ are considered with $L=5$ assuming that the available radio resources allow combining three $\left(l_{\mathrm{o}}=3\right)$ branches.

In the following, the performance of Scheme II is analyzed. At a BER of $10^{-4}$ (Figure 3.9), correlation results in about $3 \mathrm{~dB}$ SNR loss for $\sigma=4 \mathrm{~dB}$; this loss is about $7 \mathrm{~dB}$ for $\sigma=8$ $\mathrm{dB}$ and $\rho=0.8$. SNR loss is defined as the additional required SNR to maintain the same BER. Subjecting Scheme III to the same operating conditions, it is observed that for $\rho=0.8$, correlation inflicts about $2.3 \mathrm{~dB}$ loss when $\sigma=4 \mathrm{~dB}$, but this loss increases to about $6 \mathrm{~dB}$ for $\sigma=8 \mathrm{~dB}$. Although, correlation affects the performance of both schemes significantly, Scheme III is more robust than Scheme II to the tune of about $1 \mathrm{~dB}$ for the cases considered. This is due to the fact that Scheme III selects $l_{\mathrm{o}}$ branches across the ports as compared to Scheme II that concentrates on a single port. For the moderate correlations $(\rho=0.2)$, the performance advantage of Scheme III over Scheme II diminishes as depicted in Figures 3.9 and 3.10.

\subsection{Notes on Implementation and System Complexity}

The aim of the presented results is to evoke interest into the potential of the proposed hybrid macro/microdiversity architecture. In this context, a number of assumptions are made. The following section includes a discussion of the issues of practical interest which facilitated some of the assumptions as well as examination of the system complexity.

- Equal Distances and Average SNR: For analytical convenience, it is assumed that a user is equidistant from all the ports. This also ensures equal average port SNR suitable for the optimal operation of MRC. There are many practical scenarios where the equidistant assumption can be reasonably applied. One typical situation is when the macro/microdiversity architecture is used in environments with dense deployment of access ports, such as campuses (industrial, university, and others) and indoors (airports, malls, and others) [16]. In such environments, the number of deployed access ports is 
large; therefore, it is reasonable to assume that a small number of ports can be found to be at more or less the same distances from the user. For this scenario, such ports may form a "macrodiversity group" which provides even local mean SNR distributions thereby deriving the full benefit of MRC.

The whole picture by itself potentially opens other avenues of research, which might be referred to as generalized selection macrodiversity (GSM), where a subset of macrodiversity ports might be selected from the total number of deployed ports. For this reason, results for small values of macrodiversity group $(K=2,3)$ are presented where in certain scenarios the geometry allows equal distance assumption. In extreme cases however, when a user is very close to a port and relatively far from the rest, the need for extra benefit from diversity is remotely small, anyway.

Another scenario is when the access ports are deployed on the opposite sides of streets and highways. In this case, a user's trajectory is the locus of points equidistant from two ports located on the opposite sides of the road. Apart from all these scenarios, empirical studies have shown that as the amount of macrodiversity in the network increases, the system uplink becomes less sensitive to user location [73].

- Complexity: The complexities of the proposed schemes (Schemes II and III) can be estimated by comparing them with those of the conventional method. For example, the feasibility of the assumed scanning receiver has already been discussed for the classical generalized selection microdiversity [27]. Scheme II requires an additional summer circuitry; however, the associated processing cost is just negligible. Therefore, the proposed Scheme II has comparable complexity with the conventional Scheme I.

Although the macrodiversity in scheme III can be viewed as a natural extension of CDMA soft handover technique to the non-CDMA framework, the associated receiver complexity requires a more careful analysis. If the symbol rate is high, and the relative distances of the ports in a "macrodiversity group" to the CU are large, then this 
situation necessitates the use of a certain type of equalization. In a CDMA system, a relatively simple rake-type receiver can be used to combine coherently the signals in such a scenario. In a non-CDMA system, however, a more complex equalizer could be required. Intuitively, the equalization might require new formulations different from those used for single path channels. On the other hand, since the ports are fixed entities and their positions are known, synthetic link delays might be inserted in the cable connecting the ports to the $\mathrm{CU}$. This helps reduce the perceived delay spread at the $\mathrm{CU}$, and, thus, simplifies the required equalizer since the equalizer does not need a large number of taps. This strategy is borne out of the fact that in the proposed architecture, signals are delivered separately to the $\mathrm{CU}$, unlike the natural delay spread where the signals are entangled in the air.

\subsection{Chapter Conclusion}

The reverse-link performance of wireless communication systems employing a hybrid microdiversity and macrodiversity structure is studied. In the considered architecture, $K$ access ports, each carrying $L$ antennas, are deployed in a coverage region of a CU.

The contribution of this chapter can be divided into two main parts. The first part involves the derivation of error rate and outage expressions for the hybrid diversity scheme realized within the conventional macrodiversity framework (Scheme I); extensive simulations are used to validate the derived expressions. In the second part, the feasibility of SDA and RoF techniques are assumed where the signals received at ports can be transported through a broadband backbone to a CU so that the CU has $K \times L$ separable signals for each user. Novel port and branch selection strategies in the considered hybrid macro/microdiversity structure are proposed. They exhibit superior performance over the conventional method at comparable or modestly higher signal processing efforts. The issue of port correlation is also investigated. The proposed schemes can significantly combat the detrimental fading effects of 
wireless channels, however, strong port correlation significantly reduces the achievable gains.

In conclusion, the hybrid macro/microdiversity architecture with the proposed port and branch selection schemes, result in power savings which could help in cost-effective coverage extension or capacity boosting in wireless networks. 


\section{Chapter 4}

\section{Multi-antenna Cooperative Relay Networks}

The multiple antennas techniques are well studied; a number of promises in these schemes are documented [7]-[12]. The application of these techniques to mobile systems often encounter numerous practical implementation problems. For example, an element spacing of half the wavelength is required to ensure uncorrelated signals for optimal performance. The future wireless terminals are expected to be small and light. The small size feature limits the spatial separation needed by multiple antennas systems for their optimal performance. Therefore, efficient power utilization becomes a great priority in wireless terminals; the lightweight feature limits the power capability and signal processing that the terminals can support. These factors make the deployment of a large number of antennas not feasible in practice. Therefore, novel techniques for exploiting network resources through nodes cooperation, known as cooperative diversity or antenna sharing, are considered. The interest in this area is steadily growing, thus spawning a surge of publications on relaying and cooperative relaying networks which have informed most of the references in [38]-[51].

Most of the early work in the literature on relaying deals with coverage extension [61] or network capacity distribution [36, 62]. These multi-hop relaying approaches are usually used 
because of their economic advantages over cell splitting methods which are used in conventional cellular networks to increase system capacity and spectral efficiency. This chapter presents how infrastructure-based fixed relays can be used for providing spatial diversity gains for a wireless terminal which, otherwise, has limitations in the number of antennas it can bear.

As an add-on to network relays, it is demonstrated that the difficulties of using multiantenna on mobile terminals can be alleviated through cooperative use of these relays. Infrastructure - based fixed relays might have the capability to carry multiple antennas. Therefore, this chapter investigates the multi-antenna aspects of cooperative two-hop networks. Single antenna relaying is a special case of the set-up.

Threshold maximal ratio combining and threshold selection combining of these multiple antenna signals are examined and analyzed. Threshold decoding is employed as an additional measure towards combating error propagation. A related multi-antenna relay scheme is discussed in [62]. However, the following differences exist between the treatment here and that in [62]. First, the relays in [62] do not cooperate, the $L$-antenna at each relay do the cooperation instead. Second, the network capacity is used as a performance criterion.

In the next section, system model and relaying protocol are discussed. Following the endto-end (E2E) error performance formulation in Section 4.2, the error probability calculations of relay are presented in Section 4.2.2. Section 4.3 discusses how multi-antenna system could reduce the dependence of the cooperative scheme on threshold decoding. This is followed by numerical examples and discussions of E2E system performance in Section 4.4. Section 4.5 motivates possible modifications at the relay level that could be used to address the bandwidth inefficiency suffered by providing orthogonal resources for multi-relay schemes. The chapter conclusions are included in Section 4.6. 


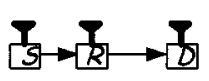

(a)

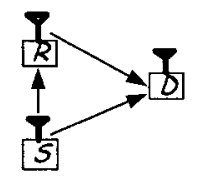

(b)

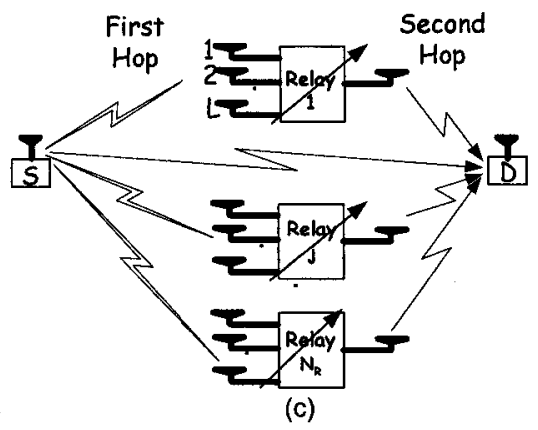

Figure 4.1: Relay networks (a) conventional fixed decode and forward (b) cooperative relaying, (c) multi-antenna cooperative relay terminals.

\subsection{System of Multi-antenna Multi-relay}

The scenario where each relay is equipped with $L$ antennas is investigated. The decode-andforward relaying strategy is adopted; i.e., the relays are of the regenerative (digital) type. Since by design, the relay is expected to be cost-efficient and of low complexity, the relay may use a subset of these $L$ antennas; in the limiting (worst) case scenario a relay uses one antenna in the so-called selection combining. In this case, only one signal detection chain is required at each relay similar to the relays which do not use multiple antennas. This explains why each relay uses only one antenna to forward as depicted in Figure 4.1. Therefore, in terms of cost, this selection diversity-based relay system does not incur any significant penalty (other than the cost of the extra antennas and selection mechanism). If the relays can utilize signals from more than one antenna, then the classical generalized selective combining (GSC) scheme can be employed.

The E2E performance of the multi-antenna multi-relay scheme is formulated in the following section. 


\subsection{Formulation of the E2E System Error Performance}

A general link in the network as a node $i$ communicating with node $j$ is defined. A node could refer to a source, relay or destination. The received signal at node $j$ can then be written as $y_{i j}=y_{i \rightarrow j}=\alpha_{i j} x_{i}+n_{j}$, where $x_{i}$ is the signal emanating from node $i$. For a relay in a multi-antenna relay network, the input-output relation of the first hop, i.e., source (node $S$ ) to relays can be expressed as

$$
\mathbf{y}_{j}=\boldsymbol{\alpha}_{S j} x_{S}+\mathbf{n}_{j}, \quad j=1,2, \cdots, N_{R},
$$

where $N_{R}$ is the total number of relays, $\mathbf{y}_{j}$ is the $L \times 1$ received vector at the $j$ th relay, $\alpha_{S j}=\left[\alpha_{S j}^{(1)}, \cdots, \alpha_{S j}^{(L)}\right]^{T}$ denotes the random channel vector with independent components which are also independent of the components in the $L \times 1$ AWGN vector $\mathbf{n}_{j}$. For a coherent scheme, perfect phase and carrier recovery is possible. Therefore, each entry $\alpha_{i j}^{(l)}$ represents the magnitude of the fade sample which follows the Nakagami distribution. The channel state information of the relay-destination links is available to the destination whereas those of the source-relay (S-R) links are known to the relays. The destination does not require any knowledge of the S-R channels.

In the second hop, the relay $j$ forwards the pre-processed signal $\left(\hat{x}_{j}\right)$ to the destination (node D) provided that the received SNR is greater than a threshold $\gamma_{t h}$. This signal is received as

$$
y_{j D}=\alpha_{j D} \hat{x}_{j}+n_{j D}, \quad j=1, \cdots, N_{R}
$$

where $\alpha_{j D}$ is the fade sample in the link between relay $j$ and destination, and $y_{j D}$ and $n_{j D}$ are the received signals and receiver noise during the reception, respectively. The model (4.2) assumes that received signals from the relays satisfy the orthogonality condition that is usually invoked in the classical literature. It implies that the relay signals do not constitute interference for each other at the destination. With the relay complexity described in Section 4.5, the received signal at the destination can be expressed in the form $y_{D}=\sum_{j=1}^{N_{R}} z_{j} \alpha_{j D} \hat{x}_{j}+n$, 
where $z_{j} \epsilon\{0,1\}$ depending on whether relay $j$ transmits $\left(z_{j}=1\right)$ or not $\left(z_{j}=0\right)$. However, the model in (4.2) is used to expose the diversity benefits of the cooperative networks thereby establishing a lower bound on the performance of the systems motivated in Section 4.5.

\subsubsection{Error Rate Analysis}

The overall end-to-end $(\mathrm{E} 2 \mathrm{E})$ error rate $P_{e, \mathrm{E} 2 \mathrm{E}}^{(\mathrm{TDF})}$ of multi-antenna multiple relays and protocol can be approximated, using an approach similar to that presented in [58] as

$$
P_{e, \mathrm{E} 2 \mathrm{E}}^{(\mathrm{TDF})}=P_{\mathrm{dec}}^{\prime} P_{\mathrm{e}, \mathrm{r}} P_{\mathrm{e}, \mathrm{p}}+P_{\mathrm{dec}}^{\prime}\left(1-P_{\mathrm{e}, \mathrm{r}}\right) P_{e, \mathrm{coop}}+\left(1-P_{\mathrm{dec}}^{\prime}\right) P_{e, \mathrm{dir}}
$$

The first and second terms represent the component due to cooperation while the third demonstrates the no-cooperation case. The first term is needed to account for possible error propagation. $P_{e, r}$ is the error probability at the relay given that the received SNR $\gamma$ is greater than $\gamma_{t h}, P_{e, \text { coop }}$ is the destination error rate when cooperation is in effect (i.e., more than one branches are combined at destination). $P_{\mathrm{dec}}^{\prime}$ is the probability that at least one relay performs decoding and forwarding, ${ }^{1} P_{e, \text { dir }}$ is the destination probability of error when no relay forwards. The impact of the number of relays have been captured by averaging over the possible cooperative scenarios $P_{e, \text { coop }}$ and $P_{\mathrm{dec}}^{\prime}$, and therefore, the error probability due to error propagation $P_{\mathrm{e}, \mathrm{p}}$ can be bounded with the worst case scenario, $P_{\mathrm{e}, \mathrm{p}} \leq 1 / 2[58]$.

The expression in (4.3) is an approximation for the following reasons: The cumulative impact of the error propagation by the relays (although small in the multi-antenna scheme) is only represented with a bound. The probability of having diversity combining at the destination is modelled with the assumption that at least one relay forwards. To get the exact value we need to consider all possible combinations of forwarding relays, which will only bring unwarranted complexity with no significant impact on the results. This is deduced from the fact that E2E system simulations match closely the performance obtained using this formulation.

\footnotetext{
${ }^{1}$ This implies there is diversity combining at destination.
} 
$P_{\text {dec }}^{\prime}$ is obtained as follows. Let $P($ relay $j$ does not forward $)=1-P($ relay $j$ does forward $)=$ $1-P_{\mathrm{DFP}, j}$. Then, $P_{\mathrm{dec}}^{\prime}$ can be expressed as

$$
P_{\mathrm{dec}}^{\prime}=1-\prod_{j=1}^{N_{R}}\left(1-P_{\mathrm{DFP}, j}\right)=\sum_{j=1}^{N_{R}}\left(\begin{array}{c}
N_{R} \\
j
\end{array}\right)(-1)^{j+1}\left(P_{\mathrm{DFP}}\right)^{j}
$$

where $P_{\mathrm{DFP}, j}=P_{\mathrm{DFP}}$, for all $j$.

In symmetric network scenarios ${ }^{2}$, the channels are considered to be balanced (same average SNR). Assuming that all the relays have the same error performance, $P_{e, \text { coop }}$ can be evaluated for equal-amplitude modulation as follows. It will be shown below that the probability of error for an $L$-branch MRC-receiver in a Nakagami- $m$ channel is given as $\frac{h \Gamma[L m+1 / 2]}{\sqrt{\pi} \Gamma[L m]} B_{\mu}[L m, 1 / 2]$. The probability that $i$ relays forward which gives rise to $(i+1)$ diversity branches at the destination is given as ${ }^{N_{R}} C_{i}\left(1-P_{\mathrm{DFP}}\right)^{N_{R^{-}}-i} P_{\mathrm{DFP}}^{i}$ where ${ }^{N_{R}} C_{i}=\frac{N_{R} !}{i !\left(N_{R}-i\right) !}$. Therefore, $P_{e, \text { coop }}$ can be expressed by weighted average over the possible cooperative scenarios, as

$$
P_{e, \text { coop }}=\frac{h}{\sqrt{\pi}} \sum_{i=1}^{N_{R}}{ }^{N_{R}} C_{i}\left(1-P_{\mathrm{DFP}}\right)^{N_{R^{-i}}} P_{\mathrm{DFP}}^{i} \frac{\Gamma[(i+1) m+1 / 2]}{\Gamma[(i+1) m]} B_{\mu}[(i+1) m, 1 / 2],
$$

where $B_{\mu}[\cdot, \cdot]$ and $\Gamma[\cdot]$ are the incomplete beta and gamma functions [80], respectively. The parameter $\lambda=q \sin ^{2}(\pi / M)$ and $M$ is the modulation constellation size. The parameters $q$ and $h$ are defined according to the modulation scheme and the nature of signal detection. Finally, $\mu=m /(m+\lambda \bar{\gamma})$.

By examining (4.3) and (4.5) it can be shown that how well the relay performs has a tremendous impact on the cooperation benefit. For instance, if $P_{\mathrm{e}, \mathrm{r}}$ is low and $P_{\mathrm{dec}}^{\prime}$ is high, one obtains the most desirable benefit from the cooperation:

$$
P_{e, \mathrm{E} 2 \mathrm{E}}^{(\mathrm{TDF})} \approx P_{e, \mathrm{coop}}
$$

The result given in (4.6) provides considerable information. First, relays always have good and reliable signals to transmit. Then, combining these signals at the destination, the full benefit

\footnotetext{
${ }^{2}$ This assumption de-emphasizes the distance-dependent received power variation. This variation factor can be incorporated by generalizing the results to branches with unequal mean received power levels in the diversity combining.
} 
of diversity can be derived because of the distributed nature of the transmitting entities. This implies that the spatial constraint that usually hampers collocated multiple antennas schemes is, thus, removed and diversity order equal to $N_{R}+1$ can be achieved. Furthermore, since the relay perfectly decodes the source information, the source appears (to the destination) as if it were at the position of the relay, hence, the path loss saving advantage is exploited. This is applicable only in the asymmetric channel scenario that incorporates the distance-dependent received power variation.

Power sharing among the transmitting entities in the network was not considered for the following reasons. First, consider the $N_{R}$ relay networks, thus, $\mathcal{P}_{i}=\mathcal{P} /\left(N_{R}+1\right), i=$ $1, \cdots N_{R}, S$ could be conservatively allocated to the relays and the source, which is intended for fairness in system comparison. $\mathcal{P}$ is the network power for the base-line scheme. However, on the average, only $\bar{N}_{R}$ number of relays have actually forwarded to destination. Therefore, the power per transmitting node should have been $\overline{\mathcal{P}}_{i}=\mathcal{P} /\left(\bar{N}_{R}+1\right)>\mathcal{P}_{i}$. In the light of this, the relationship between the average number of forwarding relays and the set decoding threshold has to be examined.

For each decoding threshold, however, the number of relays that contribute to signal detection at the destination falls in the range $\left[0, N_{R}\right]$. Then, conditioned on the $\gamma_{t h}$, the probability that $i$ among $N_{R}$ relays participates in the cooperation ${ }^{3}$ can be expressed as $P_{\mathrm{DFP}}^{i}\left(\gamma_{t h}, m\right)\left(1-P_{\mathrm{DFP}}\left(\gamma_{t h}, m\right)\right)^{N_{R}-i}$ which leads to the representation of the probability that $R_{f}=i$ out of $N_{R}$ relays forward to destination as

$$
\operatorname{Pr}\left\{R_{f}=i\right\}=\left(\begin{array}{c}
N_{R} \\
i
\end{array}\right) P_{\mathrm{DFP}}^{i}\left(\gamma_{t h}, m\right)\left(1-P_{\mathrm{DFP}}\left(\gamma_{t h}, m\right)\right)^{N_{R}-i} .
$$

With this, the average number of relays $\bar{N}_{R}$ is obtained as

$$
\bar{N}_{R}=\sum_{i=0}^{N_{R}} i\left(\begin{array}{c}
N_{R} \\
i
\end{array}\right) P_{\mathrm{DFP}}^{i}\left(\gamma_{t h}, m\right)\left(1-P_{\mathrm{DFP}}\left(\gamma_{t h}, m\right)\right)^{N_{R}-i}=N_{R} P_{\mathrm{DFP}}\left(\gamma_{t h}, m\right) .
$$

\footnotetext{
${ }^{3}$ i.e., the probability that $i$ relays have SNR greater than or equal to the decoding threshold
} 
For example, in Rayleigh fading environments and a single antenna at the relays, this average can be obtained by setting $m=1$ and $L=1$ in (2.27) or (2.29) as

$$
\bar{N}_{R}=N_{R} \exp \left(-\frac{\gamma_{t h}}{\bar{\gamma}}\right)
$$

which shows that $0 \leq \bar{N}_{R} \leq N_{R}$. It, therefore, implies that simply dividing the power among $N_{R}$ does not ensure fairness. Moreover, the power of fixed relays could come from AC supply at the station, which does not strain the users' batteries. These are the reasons why the total power has not been constrained.

\subsubsection{Relay Error Performance Analysis}

In Chapter 2 it has been shown that installing $L$ antennas yields some improvement in the decode and forward probability which in some way implies that the number of times the destination depends on a diversity signal is increased. The main thrust of threshold decoding is that the relay should decode reliably since any cooperation among the relays is dependent on this.

In threshold decoding, error performance at the relay, in the proper context, implies a conditional error. That is, the error performance (residual) given that receiver decodes only when the received SNR is greater than a certain threshold. This conditional dependence will be dropped but should be understood in this context.

The analysis proceeds with the derivation for the probability of a relay making error when a threshold is imposed. For a given received SNR $\gamma$, the probability of error for equal amplitude modulation (MPSK) can be expressed as

$$
P_{\operatorname{mpsk}}(e \mid \gamma) \approx h \operatorname{erfc}(\sqrt{\gamma \lambda})
$$

where $\lambda=q \sin ^{2}\left(\frac{\pi}{M}\right) \frac{E_{s}}{N_{0}}$. For BPSK $M=2, h=1 / 2, q=1$, and equality is satisfied, for higher MPSK constellations $h=1$. For other coherent binary transmission like orthogonal BFSK, $M=2, q=1 / 2, h=1 / 2$ [29], BFSK with minimum correlation $q=\frac{3 \pi+2}{6 \pi}$ and $M=2$. 
The combined receiver SNR can be expressed in the following form

$$
\gamma=\sum_{i=1}^{l_{\mathrm{o}}} \gamma_{i},
$$

where $l_{\mathrm{o}}=L=1$ implies no diversity,

$l_{\mathrm{o}}=1, L>1$ implies the selection combining (SC) diversity,

$l_{\mathrm{o}}=L, L>1$ implies maximal ratio combining (MRC) diversity, and finally

$1<l_{\mathrm{o}} \leq L, L>1$ represents the generalized selection combining (GSC) diversity.

The average BER is computed by averaging the conditional BER (i.e., (4.10)) over the underlying PDF. Following this trend, the error rate for a relay that blindly decodes and forwards (i.e., does not perform threshold detection) is

$$
P_{e}=\int_{0}^{\infty} P_{\operatorname{mpsk}}(e \mid \gamma) p_{\gamma}(\gamma) d \gamma
$$

For the threshold decoding, the receiver refrains from detection if the received SNR falls below the SNR threshold $\gamma_{t h}$. The probability of error at the relay is therefore calculated in the $\gamma \geq \gamma_{t h}$ regime. For the sake of simplicity, this error performance is denoted as $P_{\mathrm{e}, \mathrm{r}}\left(\bar{\gamma}, \gamma_{t h}\right)$, although it also depends on $L$ and $m$. Proceeding with the integration in (4.12)

$$
\begin{aligned}
\int_{0}^{\infty} P_{\mathrm{mpsk}}(e \mid \gamma) p_{\gamma}(\gamma) d \gamma & =\int_{\gamma=0}^{\gamma_{t h}} P_{\mathrm{mpsk}}(e \mid \gamma) p_{\gamma}(\gamma) d \gamma+\int_{\gamma_{t h}}^{\infty} P_{\mathrm{mpsk}}(e \mid \gamma) p_{\gamma}(\gamma) d \gamma \\
\int_{0}^{\infty} P_{\mathrm{mpsk}}(e \mid \gamma) p_{\gamma}(\gamma) d \gamma & =\int_{\gamma=0}^{\gamma_{t h}} P_{\mathrm{mpsk}}(e \mid \gamma) p_{\gamma}(\gamma) d \gamma+v P_{\mathrm{e}, \mathrm{r}}\left(\bar{\gamma}, \gamma_{t h}\right)
\end{aligned}
$$

upon which $P_{\mathrm{e}, \mathrm{r}}\left(\bar{\gamma}, \gamma_{t h}\right)$ can be expressed as

$$
P_{e, r}\left(\bar{\gamma}, \gamma_{t h}\right)=\frac{1}{v}(\underbrace{\int_{0}^{\infty} P_{\mathrm{mpsk}}(e \mid \gamma) p_{\gamma}(\gamma) d \gamma}_{I_{1}}-\underbrace{\int_{\gamma=0}^{\gamma_{t}} P_{\mathrm{mpsk}}(e \mid \gamma) p_{\gamma}(\gamma) d \gamma}_{I_{2}}) .
$$

Next, we evaluate $I_{1}$ and $I_{2}$ for the MRC-based detection at the multi-antenna relay in Section 4.2.3 and for SC-based in Section 4.2.4. The factor $v$ is evaluated in Appendix C.4.

First, the case in which no diversity is employed at the relay is considered. The integral 
denoted as $I_{1}$ is known for BPSK in Rayleigh fading environment, ${ }^{4}$ Nakagami fading. ${ }^{5}$ For BPSK, the integral denoted as $I_{2}$ can be expressed as

$$
I_{2}=\frac{1}{2}+\frac{\operatorname{erf}\left(\sqrt{q \gamma_{t h}}\right)-1}{2 \exp \left(\frac{\gamma_{t h}}{\bar{\gamma}}\right)}-\frac{1}{2} \sqrt{\frac{q \bar{\gamma}}{q \bar{\gamma}+1}} \operatorname{erf}\left(\sqrt{\frac{\gamma_{t h}(q \bar{\gamma}+1)}{\bar{\gamma}}}\right) .
$$

By employing $\operatorname{erfc}(x)=1-\operatorname{erf}(x)[74], I_{2}$ can be written in terms of erfc

$$
I_{2}=\frac{1}{2}\left[1-\operatorname{erfc}\left(\sqrt{q \gamma_{t h}}\right) \exp \left(-\frac{\gamma_{t h}}{\bar{\gamma}}\right)-\sqrt{\frac{q \bar{\gamma}}{q \bar{\gamma}+1}}\left(1-\operatorname{erfc}\left[\sqrt{\frac{\gamma_{t h}(q \bar{\gamma}+1)}{\bar{\gamma}}}\right]\right)\right]
$$

for Rayleigh fading.

The expressions in (4.13), (4.16) and those for $I_{1}$ are used to generate the curves in Figure 4.2 where the BER of one-antenna relay implementing threshold detection as compared to a blind relay that detects and forwards at all time is demonstrated. It is obvious that significant improvement in BER is observed and hence, the error propagation is considerably reduced.

\subsubsection{MRC-based Multi-antenna Relay and Threshold Decode-and- Forward Strategy}

The performance expression for the scenario where the MRC-based TDF relaying technique is provided. In this case, by using the PDF in (2.28), $I_{1}$ in (4.14) is obtained, thus

$$
I_{1}^{(\mathrm{MRC})}=h \int_{0}^{\infty}\left(\frac{m}{\bar{\gamma}}\right)^{L m} \frac{\gamma^{L m-1}}{\Gamma(L m)} \exp \left(-\frac{m \gamma}{\bar{\gamma}}\right) \operatorname{erfc}(\sqrt{\gamma \lambda}) d \gamma
$$

This integral can be expressed in terms of generalized hypergeometric function as

$$
I_{1}^{(\mathrm{MRC})}=\frac{h \Gamma[L m+1 / 2]}{\sqrt{\pi} \Gamma[L m+1]}\left(\frac{m}{\bar{\gamma} \lambda}\right)^{L m}{ }_{2} F_{1}\left[L m, L m+1 / 2 ; L m+1 ;-\frac{m}{\lambda \bar{\gamma}}\right] .
$$

\footnotetext{
${ }^{4} I_{1}=\frac{2+2 q \bar{\gamma}\left(1-\sqrt{\frac{2 q \bar{\gamma}+2}{q \bar{\gamma}}}\right)}{2(2+2 q \bar{\gamma})}$ for Rayleigh fading, for $q=1, I_{1}=\frac{1}{2}\left(1-\sqrt{\frac{\bar{\gamma}}{1+\bar{\gamma}}}\right)[29]$.

${ }^{5} I_{1}=\frac{\Gamma(m+1 / 2)}{2 \sqrt{\pi} \Gamma(m)} B_{x}(m, 1 / 2), x=\frac{m}{q \bar{\gamma}+m}$ as it is shown further in the chapter for Nakagami fading.
} 


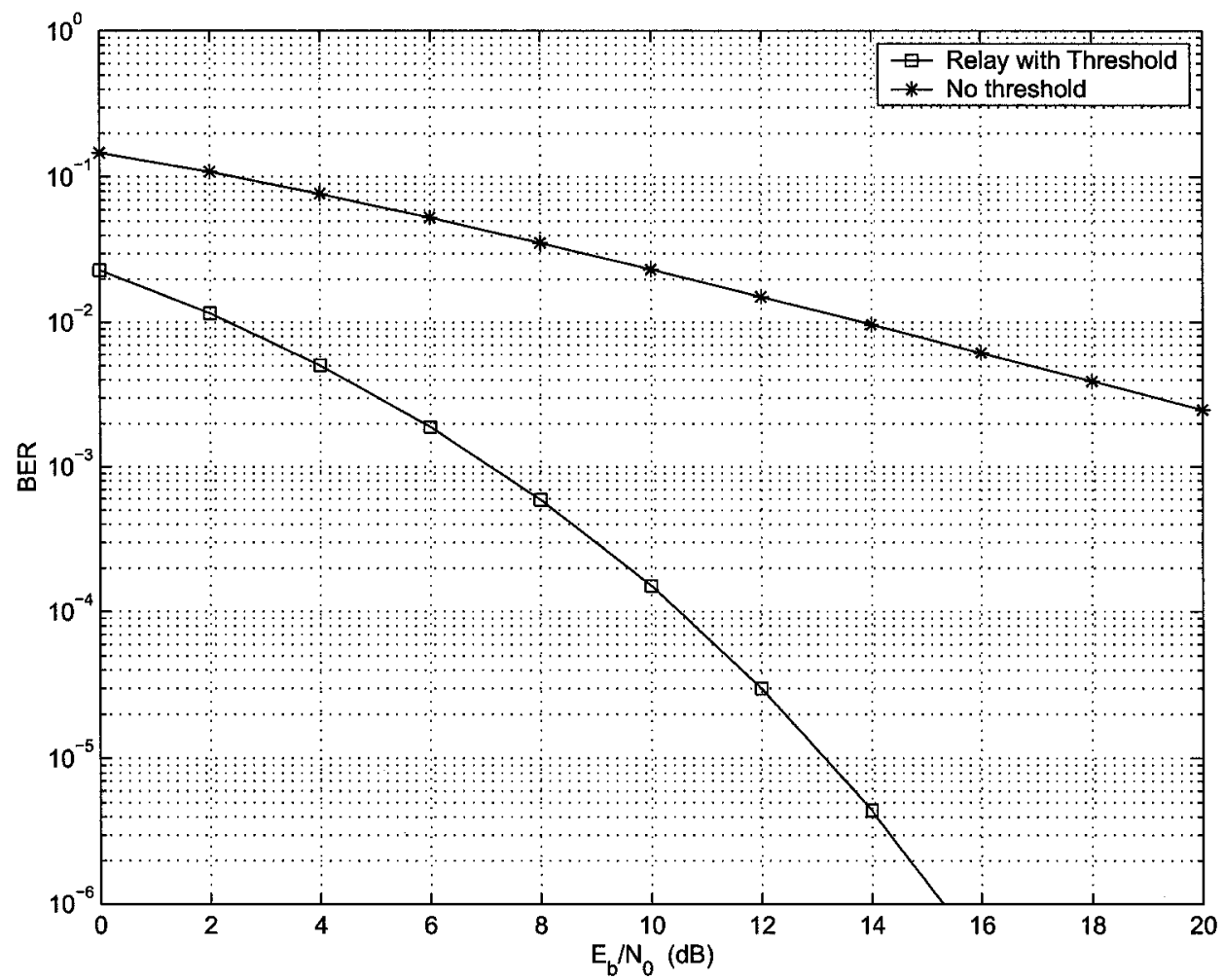

Figure 4.2: BER of relay implementing threshold decoding in single channel fading, $m=1$. 
This expression can be simplified further by using [80, pp. 1069 (9.131.1)] to express ${ }_{2} F_{1}\left[L m, L m+1 / 2 ; L m+1 ; \frac{-m}{\lambda \bar{\gamma}}\right]$ as $\left(\frac{\lambda \bar{\gamma}}{\lambda \bar{\gamma}+m}\right)^{L m}{ }_{2} F_{1}\left[L m, 1 / 2, L m+1, \frac{m}{\lambda \bar{\gamma}+m}\right]$. Finally, using [80, pp. 960 (8.391)] (4.18) can be expressed as

$$
I_{1}^{(\mathrm{MRC})}=\frac{h \Gamma[L m+1 / 2]}{\sqrt{\pi} \Gamma[L m]} B_{\mu}[L m, 1 / 2]
$$

where $\mu=\frac{m}{\lambda \bar{\gamma}+m}$. This expression is more compact than the one in [25].

To evaluate $I_{2}$ the same steps as in the derivation of $I_{1}$ are followed

$$
I_{2}^{(\mathrm{MRC})}=h \int_{0}^{\gamma_{t h}}\left(\frac{m}{\bar{\gamma}}\right)^{L m} \frac{\gamma^{L m-1}}{\Gamma[L m]} \exp \left(-\frac{m \gamma}{\bar{\gamma}}\right) \operatorname{erfc}(\sqrt{\gamma \lambda}) d \gamma
$$

It is difficult to obtain a close form expression for the exact integral of (4.20). There are two things that can be done. The first is to leave it as it is and employ numerical integration techniques available in most mathematical softwares packages; they, however, can be undesirable in most cases. The second option is to invoke some approximations that are known to be very tight. For example, $1 / 2 \operatorname{erfc}(t / \sqrt{2})$ has been shown to be well approximated by $\exp \left(-t^{2} / 2\right) /(\sqrt{2 \pi} t), t>0[86]$. Therefore, $(4.20)$ can be expressed as

$$
I_{2}^{(\mathrm{MRC})} \approx \frac{h}{\sqrt{\pi \lambda \gamma}} \int_{0}^{\gamma_{t h}}\left(\frac{m}{\bar{\gamma}}\right)^{L m} \frac{\gamma^{L m-1}}{\Gamma[L m]} \exp \left(-\left(\frac{m \gamma}{\bar{\gamma}}+\lambda \gamma\right)\right) d \gamma
$$

where a convenient expression can, then, be obtained as

$$
I_{2}^{(\mathrm{MRC})} \approx \frac{h\left(\frac{m}{\bar{\gamma}}\right)^{L m}\left(\Gamma[L m-1 / 2]-\Gamma\left[L m-1 / 2, \lambda \gamma_{t h}+\frac{m \gamma_{t h}}{\bar{\gamma}}\right]\right)}{\sqrt{\pi \lambda} \Gamma[L m]\left(\lambda+\frac{m}{\bar{\gamma}}\right)^{L m-1 / 2}}
$$

where $\Gamma[a, z]=\int_{z}^{\infty} t^{a-1} e^{-t} d t$ represents the upper incomplete gamma function. Though this approximation is tight at high SNR, it is loose at low SNR. In certain scenarios, the low SNR regime may be of interest. Therefore, we pursue the following exact expression for low SNR regime which can be combined with the high SNR regime analysis above for evaluating the system performance. First, it is noted that $\operatorname{erfc}(x)=\Gamma\left[1 / 2, x^{2}\right] / \sqrt{\pi}$. Therefore, 
$\operatorname{erfc}(\sqrt{\gamma \lambda})=\Gamma[1 / 2, \gamma \lambda] / \sqrt{\pi}$ and using $[80]$ we can write $I_{2}^{(\mathrm{MRC})}$ as

$$
I_{2}^{(\mathrm{MRC})}=h \int_{0}^{\gamma_{t h}}\left(\frac{m}{\bar{\gamma}}\right)^{L m} \frac{\gamma^{L m-1}}{\Gamma(L m)} \exp \left(-\frac{m \gamma}{\bar{\gamma}}\right)\left(1-\frac{2}{\sqrt{\pi}} \sum_{n=0}^{\infty} \frac{(-1)^{n}}{n !(2 n+1)} \lambda^{n+1 / 2} \gamma^{n+1 / 2}\right) d \gamma
$$

with further manipulations, it can be shown that

$$
\begin{aligned}
I_{2}^{(\mathrm{MRC})} & =\frac{h \Gamma[L m]-\Gamma\left[L m, \frac{m \gamma_{t h}}{\bar{\gamma}}\right]}{\Gamma[L m]} \\
& -\frac{4 h}{\sqrt{\pi}} \sum_{p=0}^{\infty} \frac{(-1)^{p}}{(p !(2 p+1))} \lambda^{p+1 / 2} \frac{\Gamma[L m+p+1 / 2]-\Gamma\left[L m+p+1 / 2, m \gamma_{t h} / \bar{\gamma}\right]}{\Gamma[L m]\left(\frac{m}{\bar{\gamma}}\right)^{p+1 / 2}} .
\end{aligned}
$$

The expressions for evaluating the performance of SC-based detection at the relay are derived next.

\subsubsection{SC-based Multi-antenna Relay and Threshold Decode-and- Forward Strategy}

In this section, the SC-based diversity at a relay is considered. In this scenario, the PDF in (2.26) is employed in computing $I_{1}^{(\mathrm{SC})}$, thus

$$
\begin{aligned}
I_{1}^{(\mathrm{sc})} & =h \int_{0}^{\infty} \frac{L}{(m-1) !} \sum_{l=0}^{L-1}(-1)^{l}\left(\begin{array}{c}
L-1 \\
l
\end{array}\right) \\
& \times \sum_{k=0}^{l(m-1)} b_{k}^{l}\left(\frac{m}{\bar{\gamma}}\right)^{m+k} \gamma^{m+k-1} \exp \left(-(l+1) \frac{m \gamma}{\bar{\gamma}}\right) \operatorname{erfc}(\sqrt{\gamma \lambda}) d \gamma \\
& =\frac{h L}{(m-1) !} \sum_{l=0}^{L-1}(-1)^{l}\left(\begin{array}{c}
L-1 \\
l
\end{array}\right) \\
& \times \sum_{k=0}^{l(m-1)} b_{k}^{l}\left(\frac{m}{\bar{\gamma}}\right)^{m+k} \int_{0}^{\infty} \gamma^{m+k-1} \exp \left(-(l+1) \frac{m \gamma}{\bar{\gamma}}\right) \operatorname{erfc}(\sqrt{\gamma \lambda}) d \gamma
\end{aligned}
$$


Using the same approach as the one employed for MRC, $I_{1}^{(\mathrm{SC})}$ can be expressed as

$$
\begin{aligned}
I_{1}^{(\mathrm{SC})} & =\frac{h L}{(m-1) !} \sum_{l=0}^{L-1}(-1)^{l}\left(\begin{array}{c}
L-1 \\
l
\end{array}\right) \sum_{k=0}^{l(m-1)} b_{k}^{l}\left(\frac{m}{\bar{\gamma} \lambda}\right)^{m+k} \\
& \times \frac{\Gamma(1 / 2+k+m)}{\sqrt{\pi}(k+m)}{ }_{2} F_{1}\left(k+m, k+m+1 / 2 ; k+m+1 ; \frac{-m(1+l)}{\lambda \bar{\gamma}}\right) .
\end{aligned}
$$

With the help of [80], the hypergeometric function can be expressed as ${ }_{2} F_{1}(k+m, k+m+$ $\left.1 / 2 ; k+m+1 ; \frac{-m(1+l)}{\lambda \bar{\gamma}}\right)=\left(\frac{\lambda \bar{\gamma}+m(1+l)}{\lambda \bar{\gamma}}\right)^{-(k+m)}{ }_{2} F_{1}\left(k+m, 1 / 2, k+m+1, \frac{m(1+l)}{m(1+l)+\lambda \bar{\gamma}}\right)$, thus, employing $[80$, pp. $960,(8.391)]$ to derive a simplified final expression

$$
I_{1}^{(\mathrm{sc})}=\frac{h L}{\sqrt{\pi}(m-1) !} \sum_{l=0}^{L-1}(-1)^{l}\left(\begin{array}{c}
L-1 \\
l
\end{array}\right) \sum_{k=0}^{l(m-1)} b_{k}^{l} \frac{\Gamma(k+m+1 / 2)}{(1+l)^{k+m}} B_{y}[k+m, 1 / 2],
$$

where $y=\frac{m(1+l)}{\lambda \bar{\gamma}+m(1+l)}$.

Finally, the derivation of $I_{2}^{(\mathrm{sc})}$ is considered.

$$
\begin{aligned}
I_{2}^{(\mathrm{SC})} & =h \int_{0}^{\gamma_{t h}} \frac{L}{(m-1) !} \sum_{l=0}^{L-1}(-1)^{l}\left(\begin{array}{c}
L-1 \\
l
\end{array}\right) \\
& \times \sum_{k=0}^{l(m-1)} b_{k}^{l}\left(\frac{m}{\bar{\gamma}}\right)^{m+k} \gamma^{m+k-1} \exp \left(-(l+1) \frac{m \gamma}{\bar{\gamma}}\right) \operatorname{erfc}(\sqrt{\gamma \lambda}) d \gamma \\
& \approx \frac{h L}{(m-1) !} \sum_{l=0}^{L-1}(-1)^{l}\left(\begin{array}{c}
L-1 \\
l
\end{array}\right) \\
& \times \sum_{k=0}^{l(m-1)} b_{k}^{l}\left(\frac{m}{\bar{\gamma}}\right)^{m+k} \frac{\left(\Gamma[m+k-1 / 2]-\Gamma\left[m+k-1 / 2, \lambda \gamma_{t h}+\frac{\left.m(l+1) \gamma_{t h}\right]}{\bar{\gamma}}\right]\right)}{\sqrt{\pi \lambda}\left(\lambda+\frac{m(l+1)}{\bar{\gamma}}\right)^{m+k-1 / 2}} .(1
\end{aligned}
$$

As with (4.22) for MRC, this approximation is only tight at high SNR. Therefore, it is necessary to find an expression for the low SNR regime. The steps to the final solution are almost the same as with the MRC derivation. Without repeating those steps, the final exact 
expression is given as

$$
\begin{aligned}
I_{2}^{(\mathrm{sC})} & =h \int_{0}^{\gamma_{t h}} \frac{L}{(m-1) !} \sum_{l=0}^{L-1}(-1)^{l}\left(\begin{array}{c}
L-1 \\
l
\end{array}\right) \\
& \times \sum_{k=0}^{l(m-1)} b_{k}^{l}\left(\frac{m}{\bar{\gamma}}\right)^{m+k} \gamma^{m+k-1} \exp \left(-(l+1) \frac{m \gamma}{\bar{\gamma}}\right) \operatorname{erfc}(\sqrt{\gamma \lambda}) d \gamma
\end{aligned}
$$

Therefore,

$I_{2}^{(\mathrm{sC})}=$

$$
\begin{gathered}
\frac{h L}{(m-1) !} \sum_{l=0}^{L-1}(-1)^{l}\left(\begin{array}{c}
L-1 \\
l
\end{array}\right) \sum_{k=0}^{l(m-1)} b_{k}^{l}\left(\frac{1}{1+l}\right)^{(k+m)}\left(\Gamma[k+m]-\Gamma\left[k+m, \frac{m(1+l) \gamma_{t h}}{\bar{\gamma}}\right]\right. \\
\left.-\frac{4 h}{\sqrt{\pi}} \sum_{p=0}^{\infty} \frac{(-1)^{p}\left(\frac{m}{\lambda \bar{\gamma}}\right)^{-(p+1 / 2)}}{p !(2 p+1)} \frac{\Gamma[k+m+p+1 / 2]-\Gamma\left[k+m+p+1 / 2, m(1+l) \gamma_{t h} / \bar{\gamma}\right]}{(1+l)^{p+1 / 2}}\right) .
\end{gathered}
$$

Figure 4.3 shows the performance of a relay with $L$ diversity antennas that also puts a threshold on the decodable received SNR. The analytical results are the solid curves and the simulations are denoted by a star, square, and circle. The Nakagami parameter value is 1 ( $m=1$, Rayleigh fading). The cases where there is no threshold decoding imposed are also shown for comparison purposes. It is observed that the threshold improves the error performance, which of course, is expected by intuition. However, what intuition fails to reveal, is how much of improvement is obtained from this deployment and the impact of the various factors involved. With this information, a system designer can be guided on selecting the best possible choice of antenna deployment strategies, the number of antenna to deploy, etc. One important observation from these results is that in classical diversity analysis, the diversity gain moving from one antenna to two is usually significant, it is always the largest incremental gain. It is observed that this gain drastically decreases in the threshold decode case. Further, in Figure 4.4, error performance is also shown for the cases where the Nakagami parameter $m$ is 2,6 .

Figure 4.5 (for $m=1$ ) and Figure 4.6 (for $m=2$ ) show the error performance of MRC- 


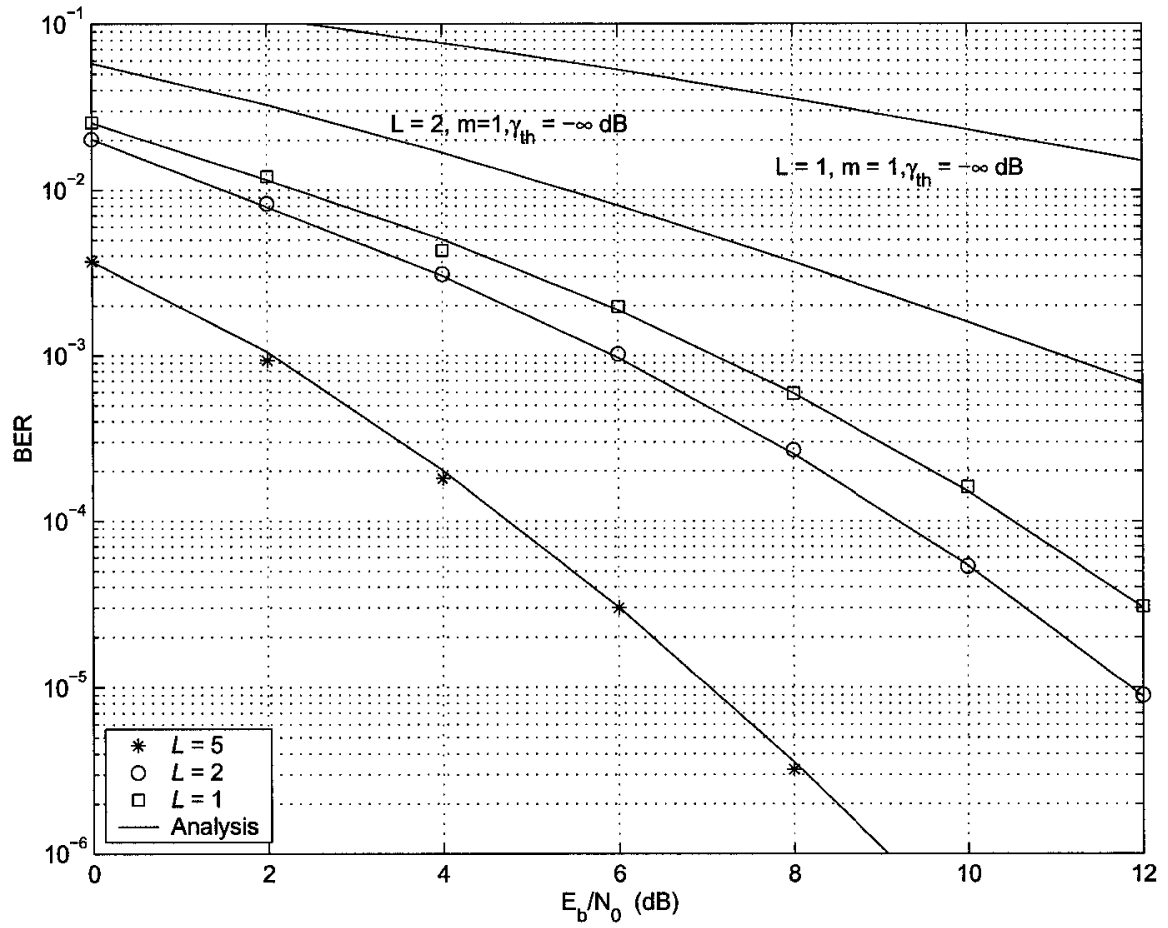

Figure 4.3: BER of MRC-based TDF relay in fading channel, $m=1$ : Simulation and Analysis. 


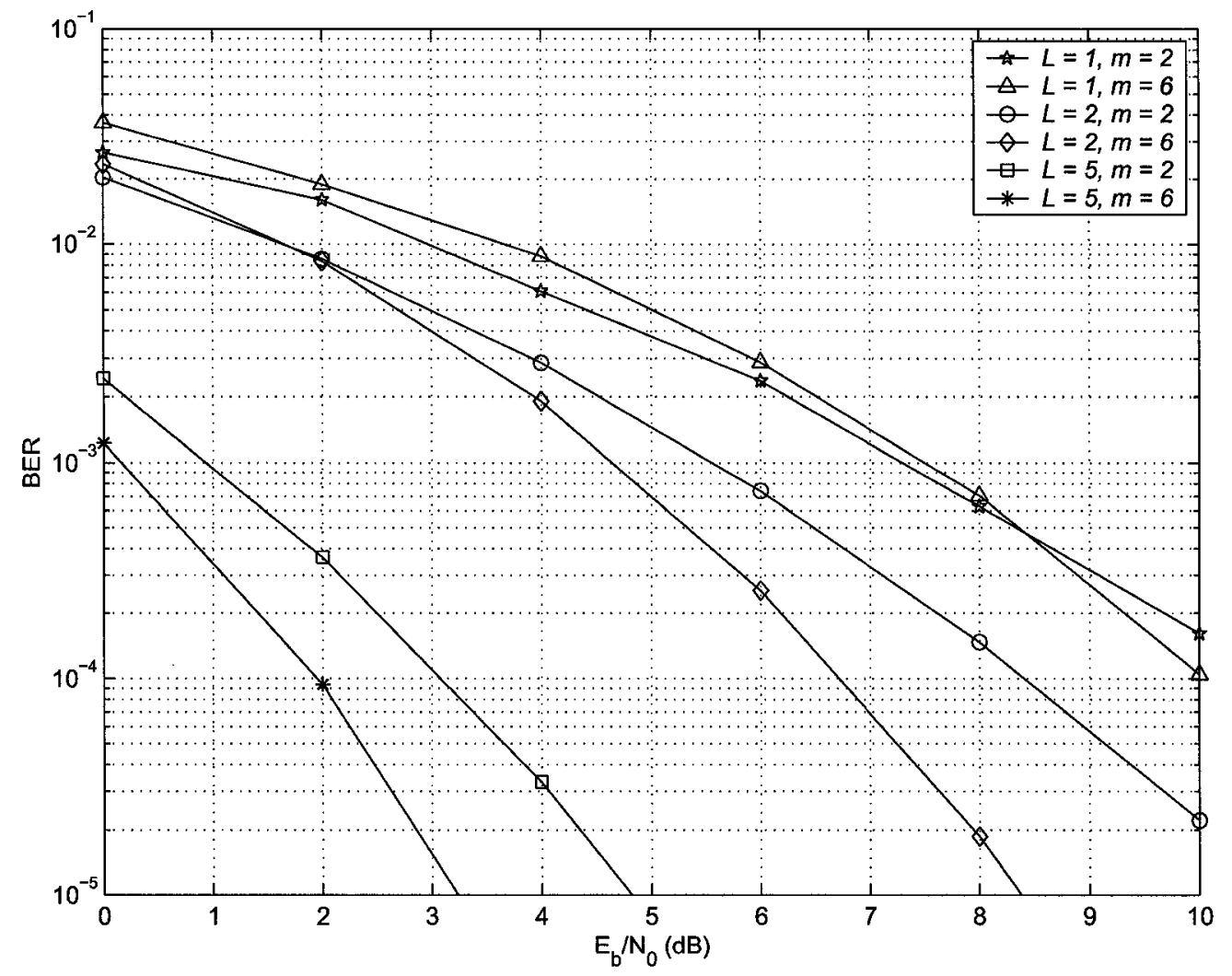

Figure 4.4: BER of MRC-based TDF relay in Nakagami fading, $m=2,6$. 


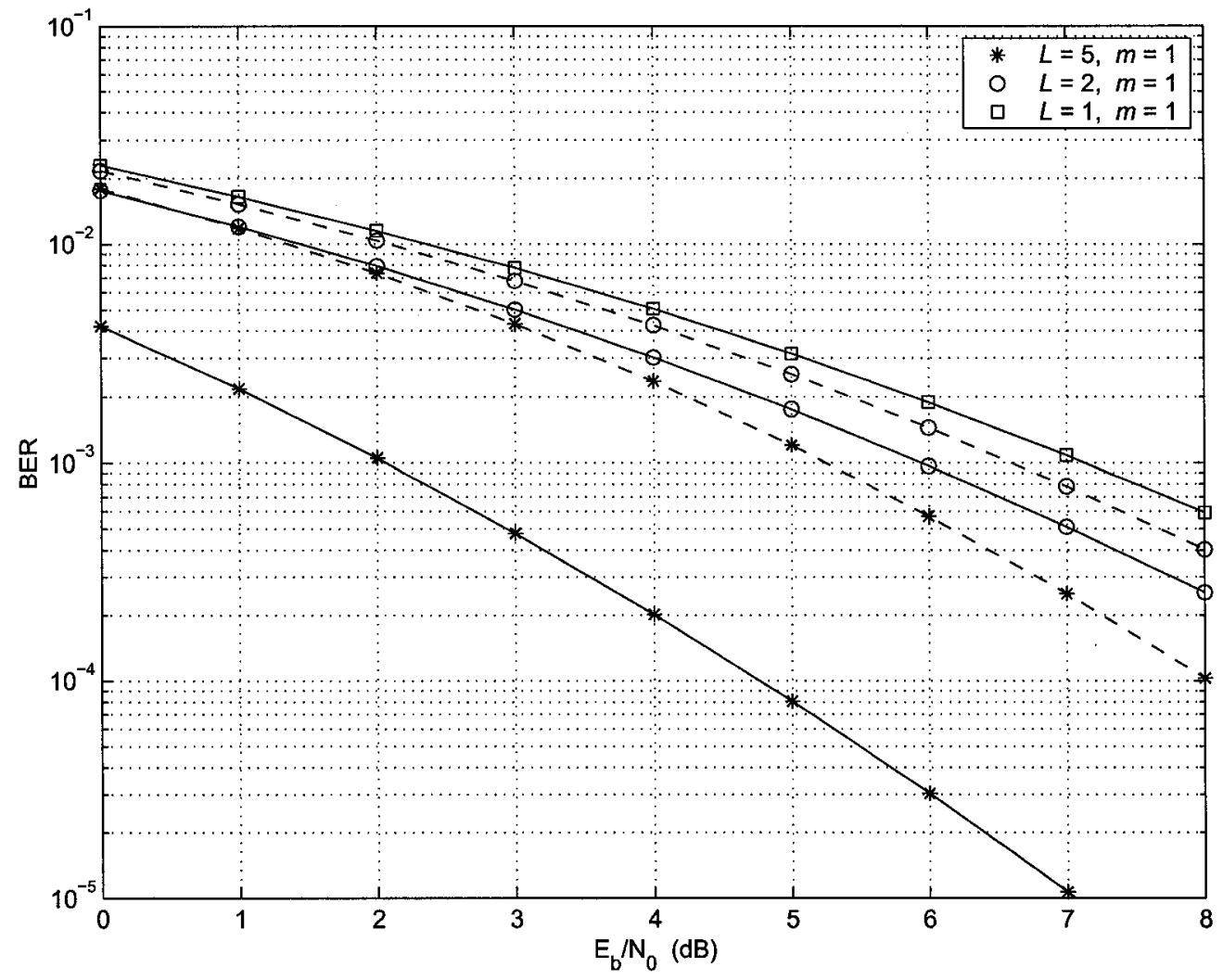

Figure 4.5: Error performance comparison of MRC-based vs SC-based TDF relay in fading channel, $m=1$ : MRC solid curves, SC the broken curves.

based vs SC-based relay, implementing DF protocol for $L=1,2$ and 5 . The expressions (4.14), (4.27), and (4.29) are used for the SC-based and (4.14), (4.19), and (4.24) used for the MRC-based relaying. The validity of the expressions for the error probability of MRCand SC-based relay is confirmed through simulations (Figures 4.3 and 4.7) where a strong agreement is observed.

Before proceeding further, threshold value selection for the TDF protocol is briefly discussed. The impact of the set threshold on the E2E system performance is investigated. This investigation also sheds lights on influence of the multiple antennas on TDF relaying. 


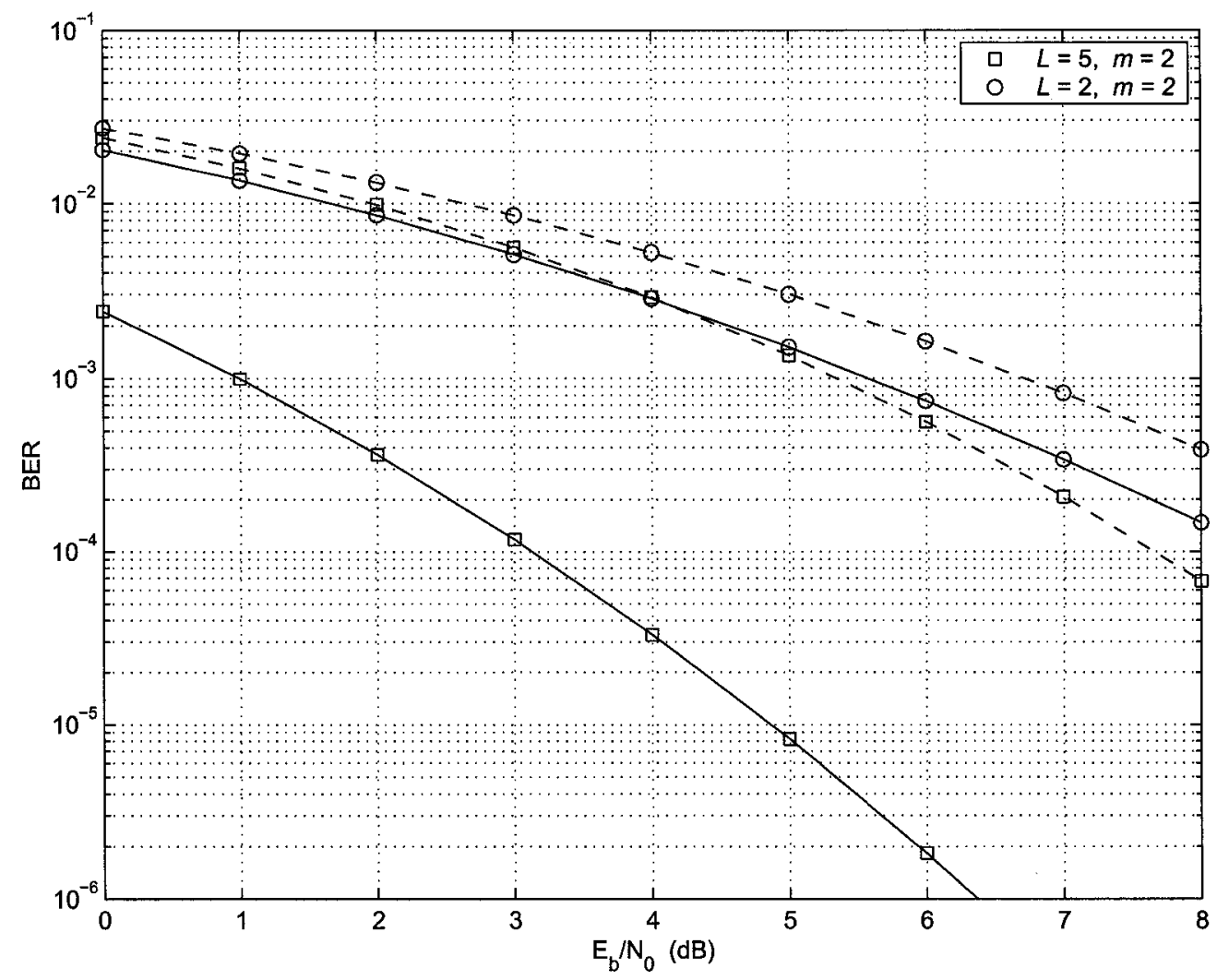

Figure 4.6: Error performance comparison of MRC-based vs SC-based TDF relay in fading channel, $m=2$ : MRC solid curves, SC the broken curves. 


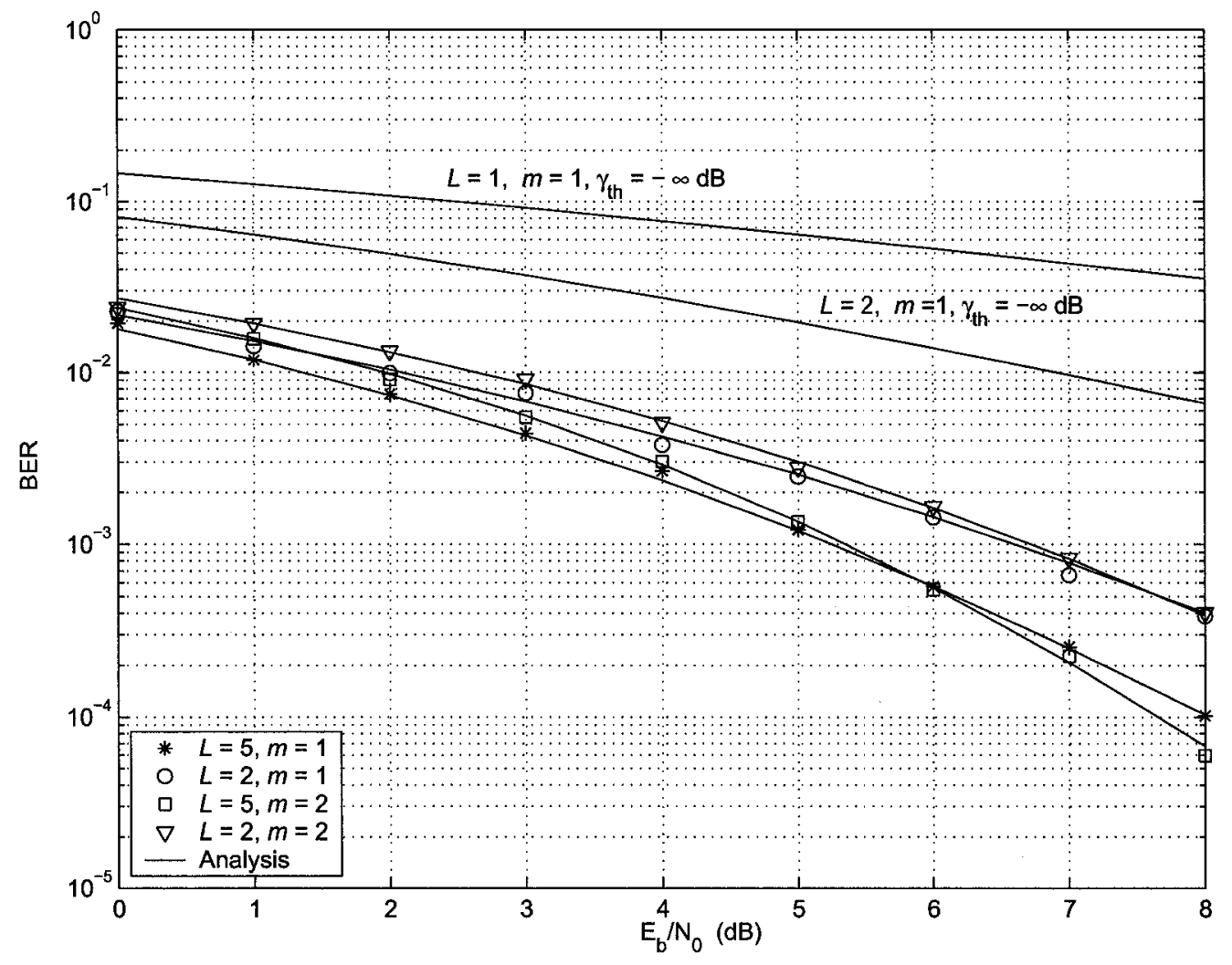

Figure 4.7: Analytical and simulated error performance of SC-based TDF relay in Nakagami fading $(m=1,2)$ and $L=1,2,5$ : solid curves represent the analysis, the symbols relate to the simulations. 


\subsection{Threshold Decode-and-Forward Protocol vs Multi- ple Antennas: Which Way?}

Whether multiple antennas at fixed relay station replace the adaptive DF relay transmission protocol is discussed in this section of the chapter. For the purpose, simple cooperative relaying scheme (one terminal cooperation) with TDF protocol and one without (but the relay is equipped with dual antennas) is investigated. First, it is demonstrated that the threshold in TDF has to be carefully selected, especially in cases where $L \leq 2$. Figures 4.8, 4.9, and 4.10 show the variation of the BER with the threshold for different network configurations and various average SNRs. These figures present some ideas on how to select the 'optimum' threshold. Furthermore, Figure 4.10 demonstrates that multiple antenna at a relay can relieve the cooperation's dependence on the threshold decoding protocol, especially when there are few relays and many antennas at these relays. In this scenario, it is preferred that a threshold is not applied at all (Figure 4.10).

Observe that from these figures, a heuristic choice for the threshold can be made as $\gamma_{t h} \approx$ $\sqrt{2 \bar{\gamma} \mathrm{dB}}$ which corresponds to the setting used in [58] for simple single antenna single relay network. It is important to state that this setting does not generalize for the relay networks examine in this thesis. A comprehensive study is required to determine how the optimum threshold depends on the networks and network parameters.

Figure 4.11 shows the E2E performance for single and dual antennas with TDF. The following observations can be made (Figure 4.11):

- For one cooperating fixed relay, multiple antennas could eliminate the need for threshold decoding, by looking at $\left(N_{R}=1, L=2, \gamma_{t h}=-\infty \mathrm{dB}, \mathrm{MRC}\right)$ with $\left(N_{R}=1, L=1\right.$, TDF, and $\left.N_{R}=2, L=1, \mathrm{TDF}\right)$.

- With single antenna parallel relays, full detection at relays is not to be performed, which is in agreement with reported findings in the literature [45]. However, with dual antennas 
on two parallel relays this restriction is relaxed. This is arrived at by comparing $\left(N_{R}=\right.$ $\left.2, L=2, \gamma_{t h}=-\infty \mathrm{dB}, \mathrm{MRC}\right),\left(N_{R}=2, L=1, \mathrm{TDF}\right)$ and $\left(N_{R}=2, L=1, \gamma_{t h}=-\infty\right.$ $\mathrm{dB})$, where performance superiority follows in this order.

- With two antennas at the relay, the E2E diversity order for the single relay node cooperation is enhanced over that of a single antenna employing, the threshold decoding protocol. Comparing the slope of $\left(N_{R}=1, L=2, \gamma_{t h}=-\infty \mathrm{dB}, \mathrm{MRC}\right)$ and $\left(N_{R}=1, L=1\right.$, $\mathrm{TDF}$ ), where the former indicates superior performance.

- Using SC- promises an excellent trade-off point in terms of performance and complexity, by comparing $\left(N_{R}=2, L=2, \gamma_{t h}=-\infty \mathrm{dB}, \mathrm{MRC}\right),\left(N_{R}=2, L=2, \gamma_{t h}=-\infty \mathrm{dB}\right.$, $\mathrm{SC})$, and $\left(N_{R}=2, L=1, \mathrm{TDF}\right)$, where, in this case, the SC might be even less complex than TDF.

\subsection{Numerical Performance Illustration of Multi-antenna Multi-relay}

The analysis starts with single antenna at the relays, where simulations and analytical results are compared in Figure 4.12. Figure 4.12 (a) shows the curves for $N_{R}=1,2,4, L=1$, and $m=1$, while Figure 4.12 (b) shows some selected cases for $m=2,6$. It is noticed that the simulations results (denoted by a square, circle and asterisk) are in strong agreement with the analysis (the solid curves).

Figure 4.13 shows the performance of the multi-antenna relay in symmetrical networks for different number of antennas $(L)$ at the relay station. Performance curves for the cases $L=$ 1,2,4 and Nakagami parameters $m=1,2,6$ are indicated. The performance of conventional relaying (see network relay deployment, Figure 4.1 (a)) is as well shown. Figure 4.13(a) shows the performance for $(L=1)$; Figure 4.13 (b) for $(L=2)$; and Figure 4.13 (c) for $(L=4)$. The 


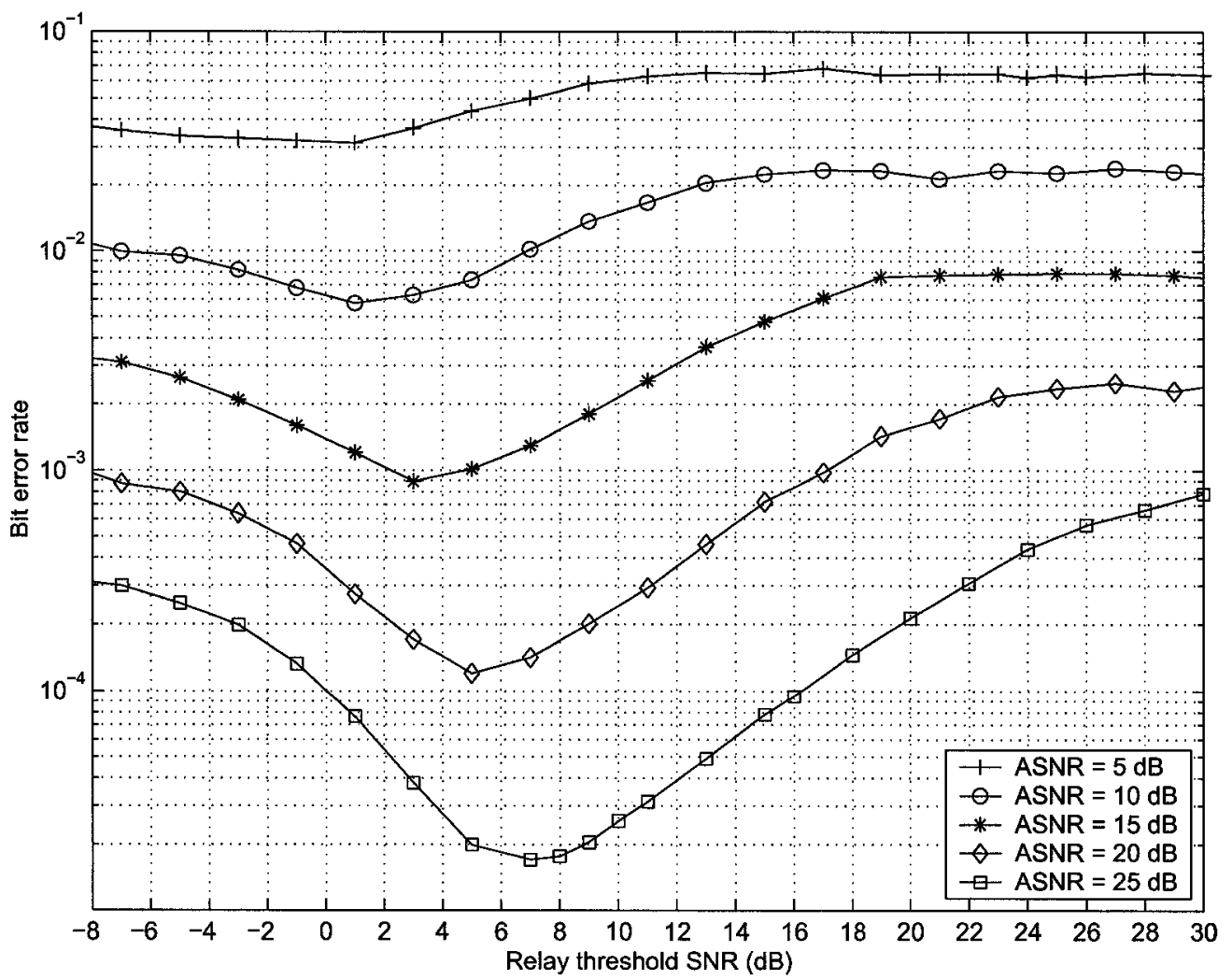

Figure 4.8: E2E BER as a function of a relay decoding threshold $\left(N_{R}=1, L=1\right)$. 


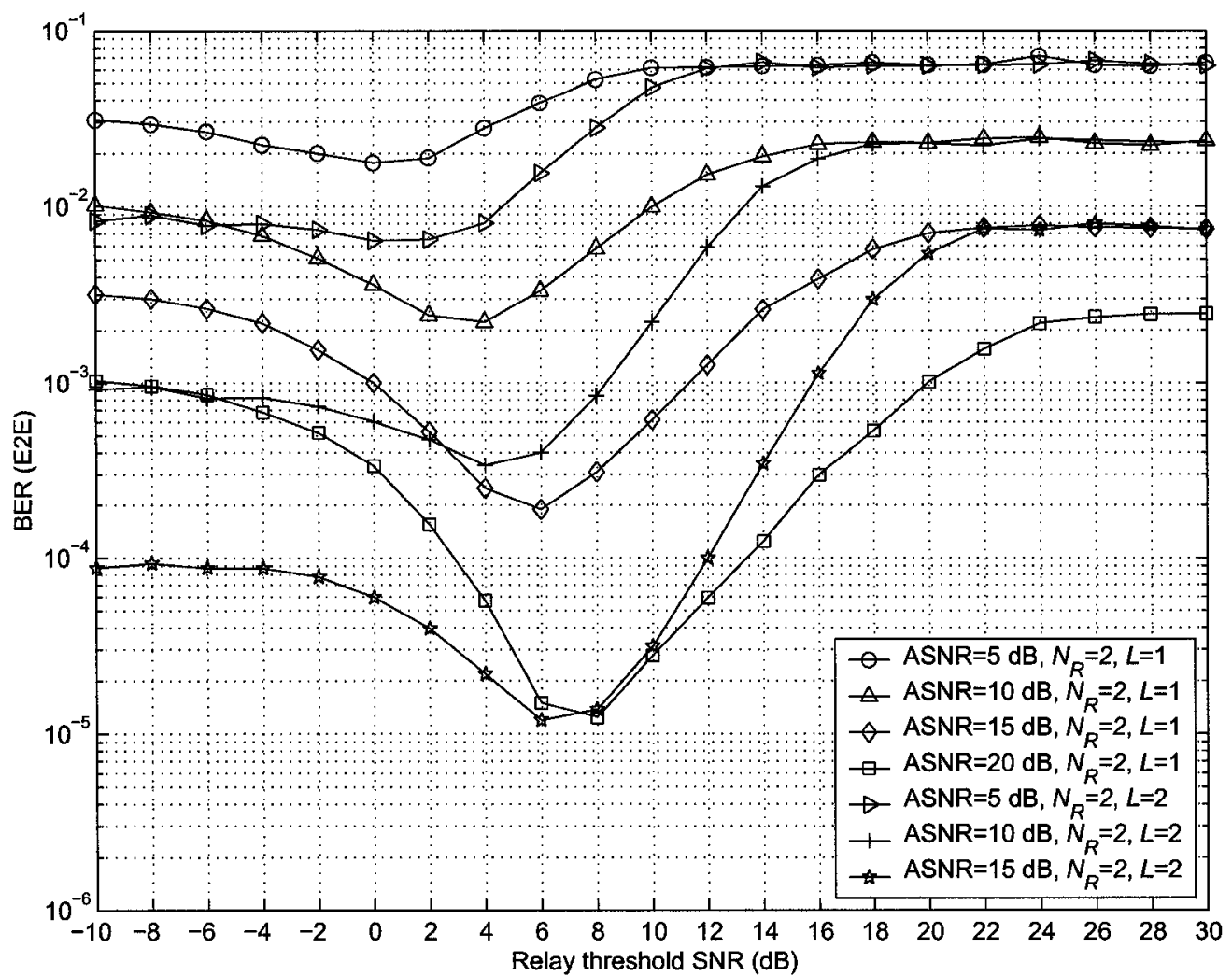

Figure 4.9: E2E BER as a function of relay decoding threshold $\left(N_{R}=2\right.$, and $\left.L=1,2\right)$. 


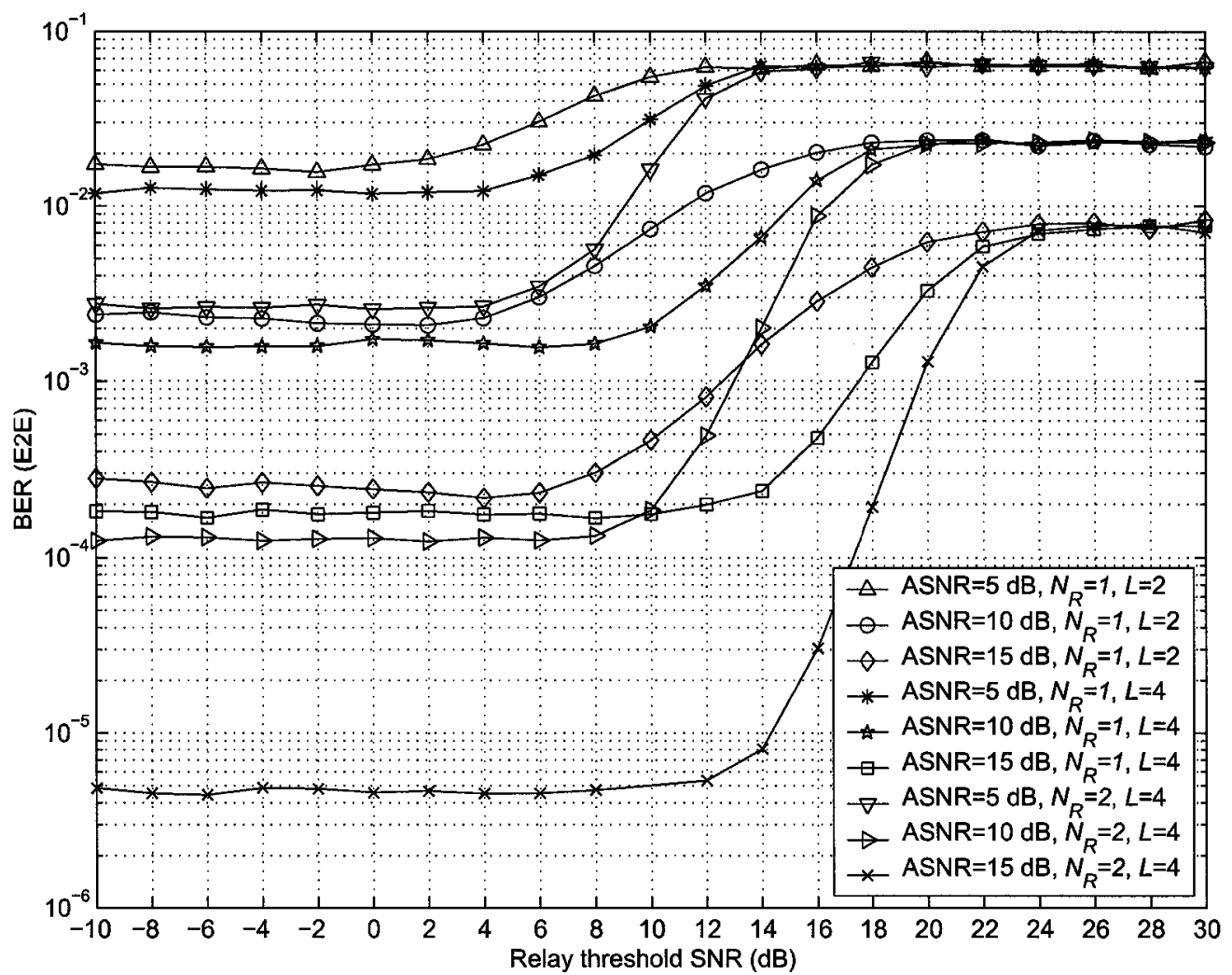

Figure 4.10: E2E BER as a function of a relay decoding threshold $\left(N_{R}=1,2\right.$, and $\left.L=2,4\right)$. 


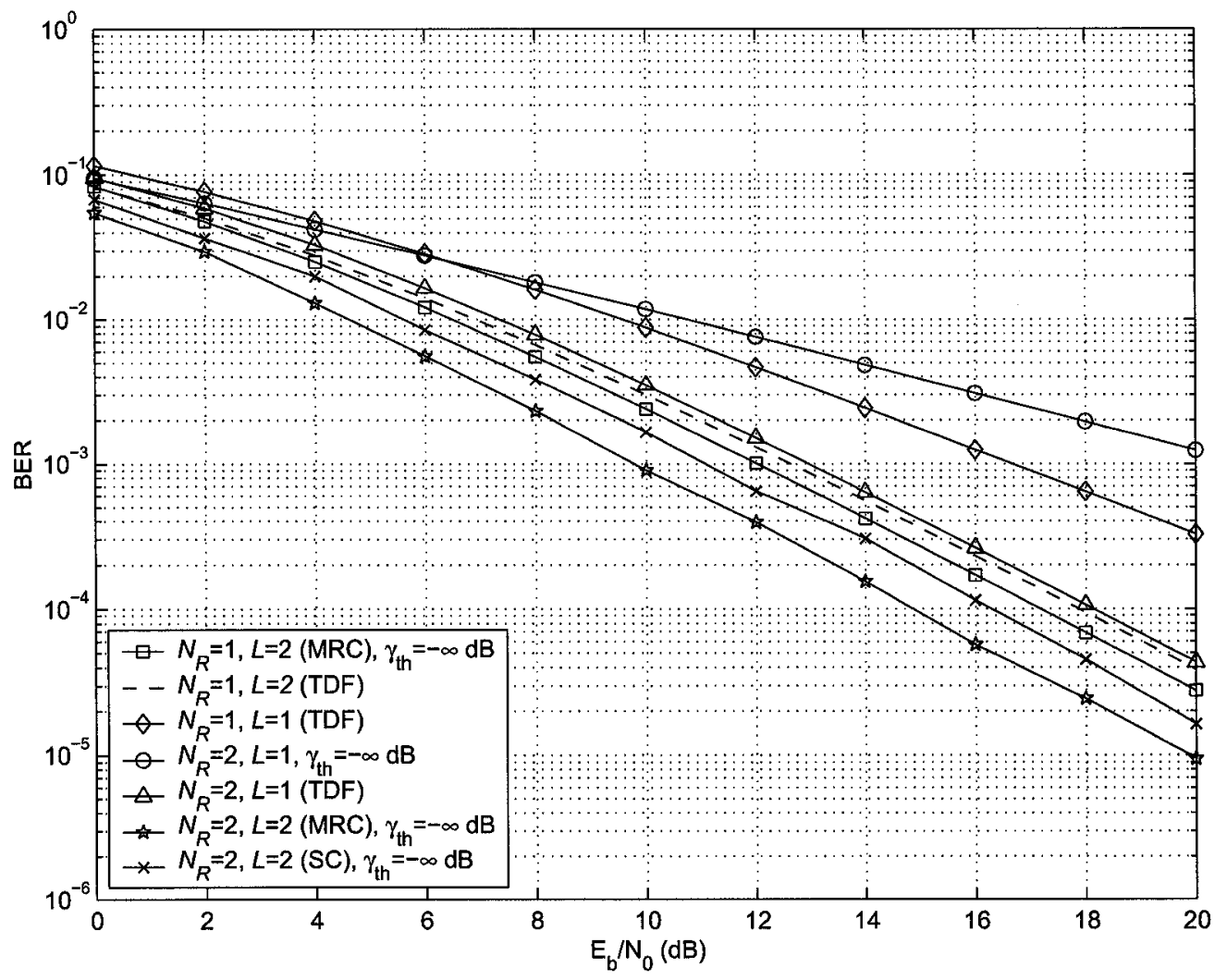

Figure 4.11: E2E BER comparison for one cooperating relay, threshold vs multiple antennas. 


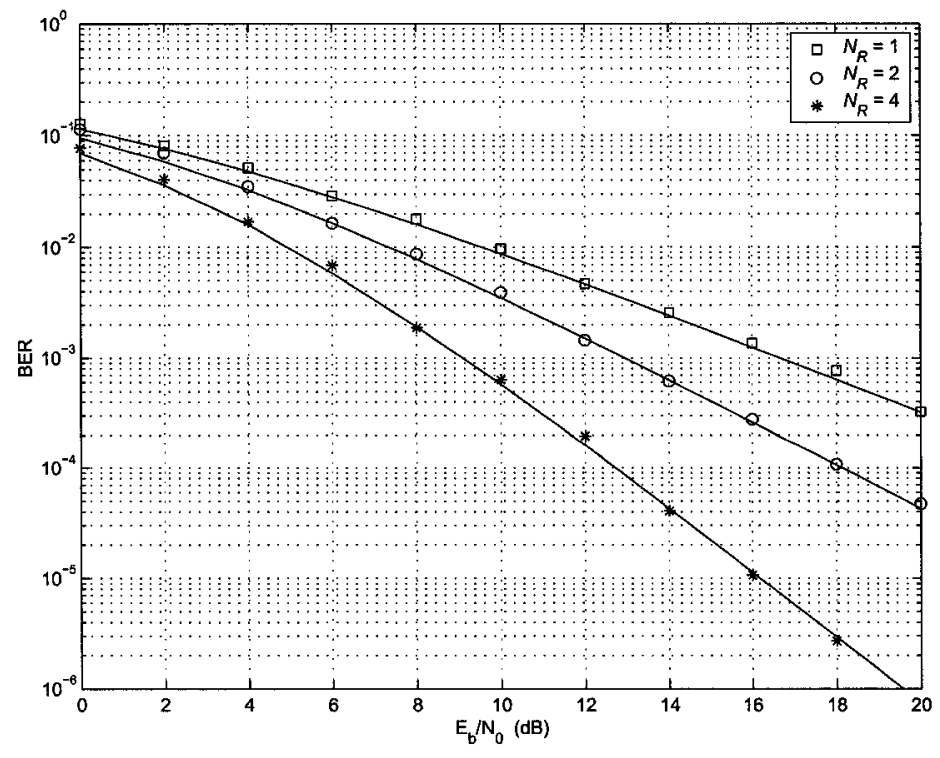

(a) $N_{R}=1,2,4, m=1$

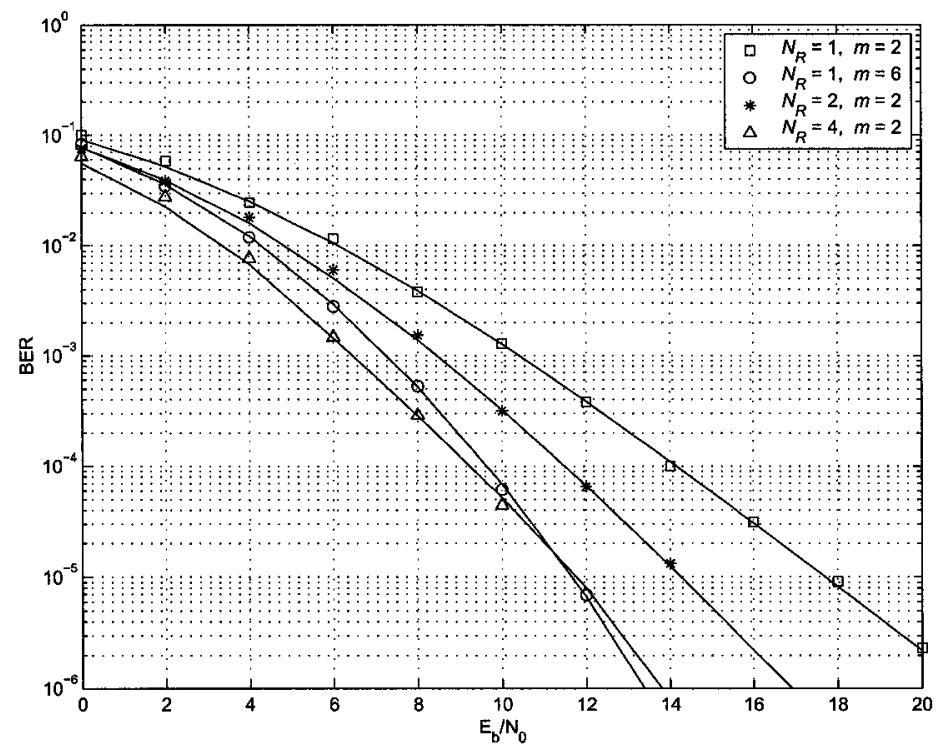

(b) $N_{R}=1,2,4, m=2$, and $N_{R}=1, m=6$.

Figure 4.12: The E2E BER of single antenna $(L=1)$ parallel relays with threshold decoding in Nakagami fading: Analysis (solid curves) and Simulation (symbols). 
relays utilize MRC combining; BPSK modulation is used in all the links. For those curves, it has been assumed that the fading distributions between the tripartite (source to relay, source to destination, and relay to source) are the same.

The evaluation of system performance for non-identical fading distributions scenarios is straightforward. For instance, if a fixed relay is positioned in such a way that it sees LOS to the source and NLOS to the destination. Since, the source would most likely have NLOS to destination, the source-relay channel can be modelled with a suitable $m$, using the Nakagami$m$ to Ricean $\mathcal{K}$-factor transformation. The relay-destination and source-destination channels are both modelled using the Rayleigh distribution $(m=1)$.

Figure 4.13 (a)-(c) shows that the multi-antenna relay systems yield considerable gains over the conventional form of relaying. The SNR requirements at an error rate of $10^{-2}$ are compared. The following gains are obtained over the conventional relaying; $10.5 \mathrm{~dB}(L=1), 13$ $\mathrm{dB}(L=2)$, and $14.5 \mathrm{~dB}(L=4)$ for $m=1$. These gains represent a significant improvement. Further, at $\mathrm{BER}=10^{-3}$, the dual antenna case exhibits about $4 \mathrm{~dB}$ superiority over the single antenna case; when four antennas are deployed, this gain is about $5 \mathrm{~dB}$. For $m=2$. However, the gain of dual antennas over the single antenna is about $1.5 \mathrm{~dB}$ while that of four antennas is $2.5 \mathrm{~dB}$. From these cases, it can be deduced that more diversity gain is obtained for a multi-antenna system in Rayleigh $(m=1)$ than in less scattering channels $(m>1)$ which again confirms the notion of the advantages of rich scattering environments. Furthermore, it seems that in dual antenna cases, the necessary diversity is already acquired, and only marginal gains are observed for an increasing number of antenna elements. In this relation, a dual antenna at the relay might be strongly recommended as the overall diversity order is improved with the dual antenna deployment.

Figure 4.14 shows the performance of 2-relay network $\left(N_{R}=2\right)$. This system is compared for a different number of antennas at a $B E R=10^{-4}$. In Rayleigh fading, it is observed that a gain of $6 \mathrm{~dB}$ is achieved for $N_{R}=2, L=2$ over $N_{R}=2, L=1$. This gain increases only 


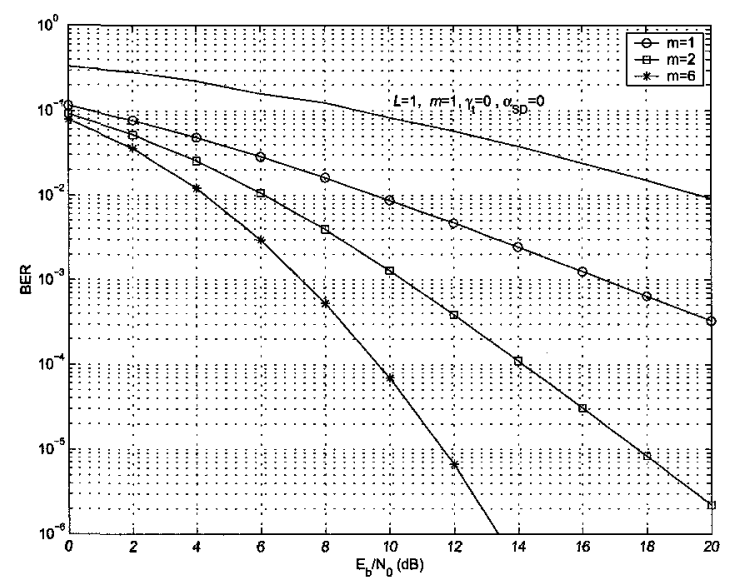

(a) $N_{R}=1, L=1$

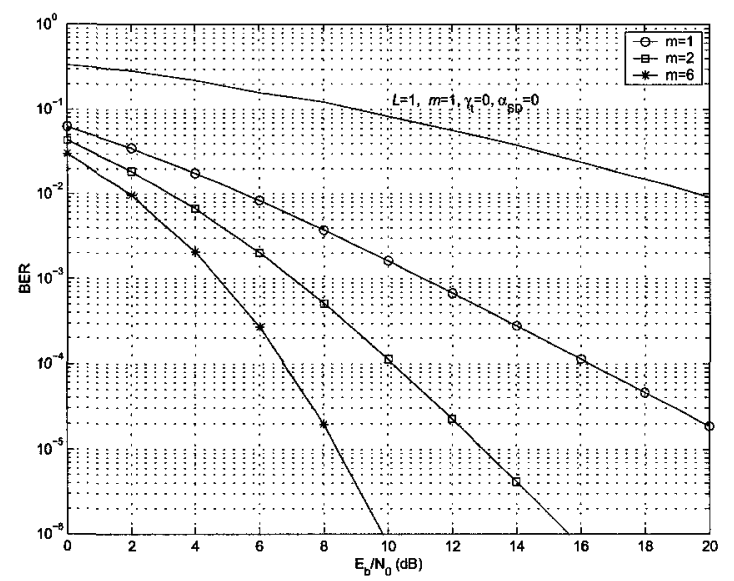

(c) $N_{R}=1, L=4$

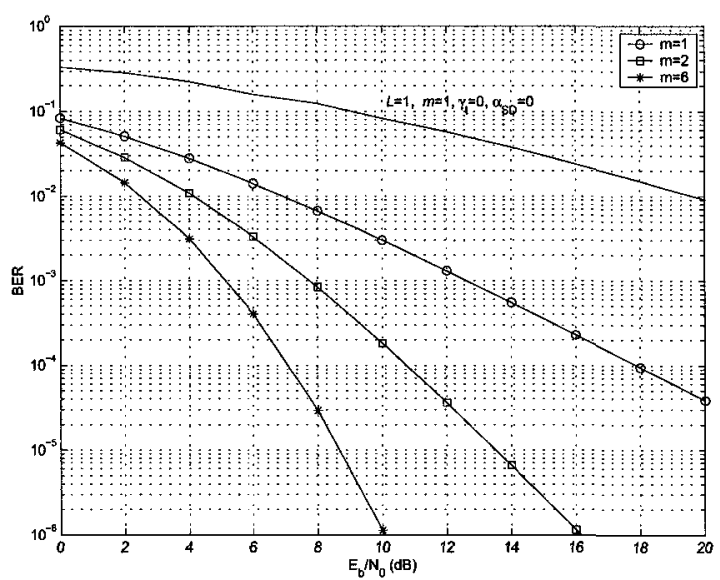

(b) $N_{R}=1, L=2$

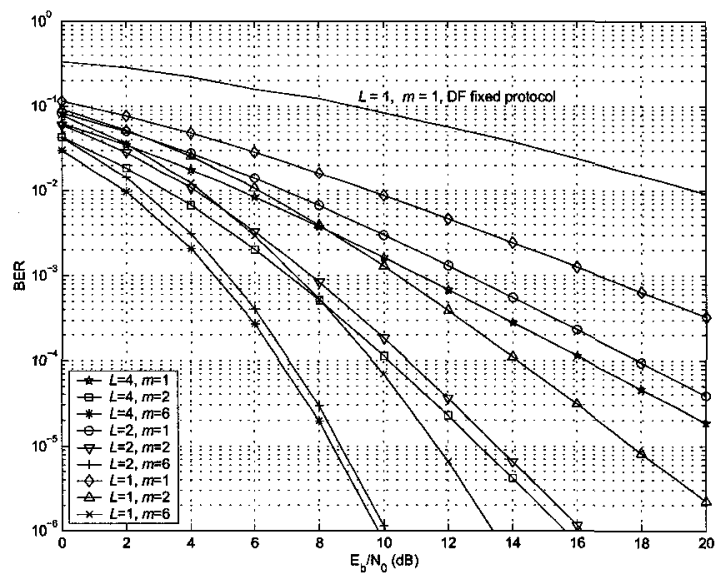

(d) $N_{R}=1, L=1,2,4$

Figure 4.13: BER performance of MRC-based multi-antenna TDF relay in Nakagami fading, $m=1,2,6$ and $N_{R}=1$. 
marginally to $7.5 \mathrm{~dB}$ when the number of antennas is increased to 4 (i.e., $N_{R}=2, L=4$ ) which buttresses the point that with dual antennas, the necessary gain is almost derived. In the less severe fading cases $(m>1)$, reduced gains are observed. For example, for $m=2$, a gain of $3.5 \mathrm{~dB}$ is obtained for $L=2$, and $4.7 \mathrm{~dB}$ for $L=4$. The trends observed in Figures 4.13 and 4.14 are generally seen for the multi-antenna 4-relay network. The performance of this system is shown in Figure 4.15.

At this point it is necessary to compare some of the system architectures (Table 4.1). The cases presented in these tables are extracted from the figures (Figures 4.14 and Figure 4.15) to make it easy for comparison. SNR required for an error rate $10^{-4}$ for different parameters, $L$ and $m$ is considered. It can be observed (Table 4.1) that $N_{R}=2, L=2$ outperforms $N_{R}=4, L=1$, although, the number of detection chains required in both configurations is the same. However, the case of $N_{R}=2, L=2$ has an additional advantage of system deployment cost over $N_{R}=4, L=1$ where it can be argued that it is considerably cheaper to install an additional antenna at the relay than deploying extra relays. When a dual antenna is deployed, with $N_{R}=4$, it is observed that $N_{R}=4, L=2$ outperforms $N_{R}=2, L=4$ by about $1.6 \mathrm{~dB}$ for $m=1$ and about $0.4 \mathrm{~dB}$ for $(m=2)$. The gain of $N_{R}=4, L=2$ over $N_{R}=2, L=4$ has to be carefully viewed. If the cost of these two systems is considered (deploying two relay stations as opposed to installing two antennas on relay stations) this gain as inconsequential can be quickly dismissed, i.e., it is too small in comparison to the concomitant cost and complexity over the $N_{R}=2, L=4$. In fact, as observed, this gain disappears at a less severe fading. In conclusion, the system configuration $N_{R}=2, L=4$ should be preferred to $N_{R}=4, L=2$ for deployment, considering the capital involvement on land acquisition, labor, maintenance, etc. Similar conclusions can be derived in other scenarios.

Figure 4.16 serves to compare MRC and SC with threshold decode-and-forward. First, it is necessary to compare the techniques for the Rayleigh fading channel environment ( $m=$ 


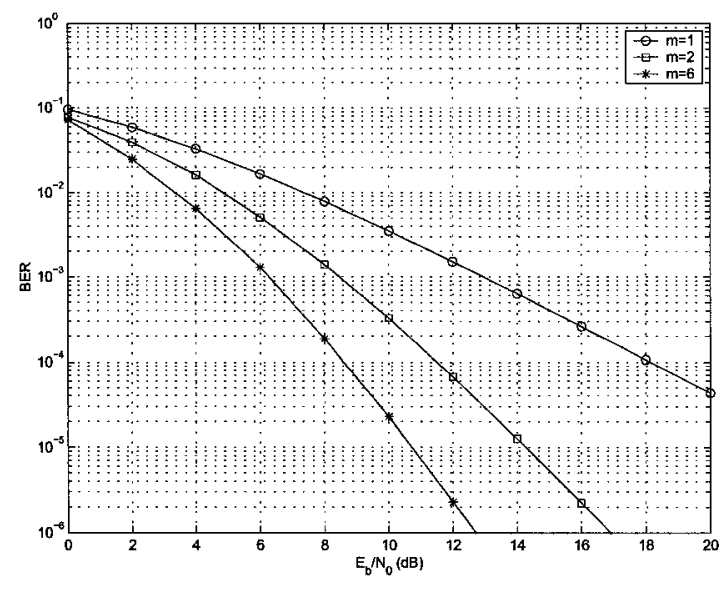

(a) $N_{R}=2, L=1$

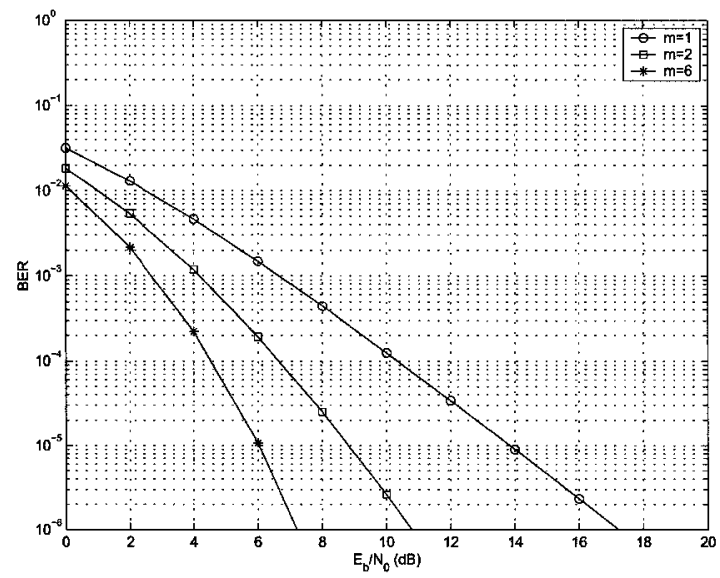

(c) $N_{R}=2, L=4$

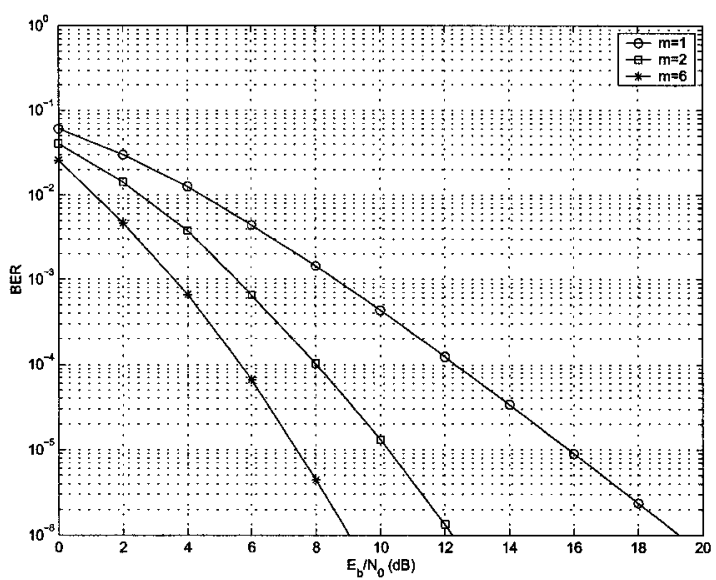

(b) $N_{R}=2, L=2$

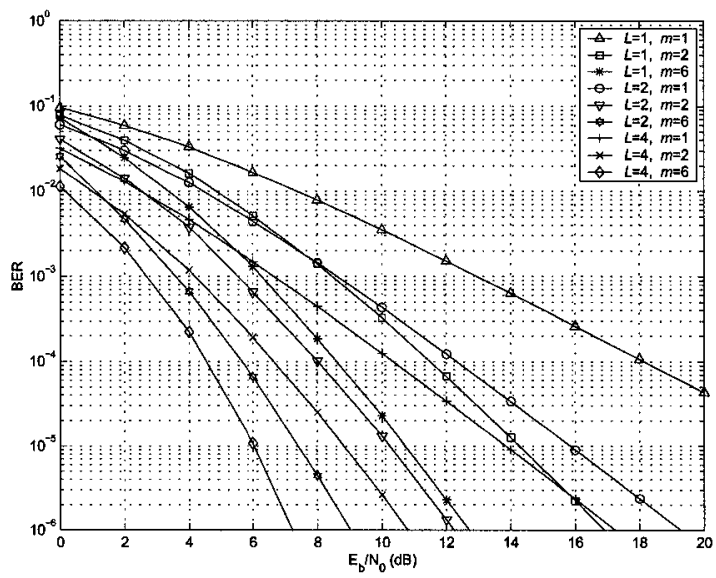

(d) $N_{R}=2, L=1,2,4$

Figure 4.14: BER performance of MRC-based multi-antenna TDF relay in Nakagami fading, $m=1,2,6$ and $N_{R}=2$. 


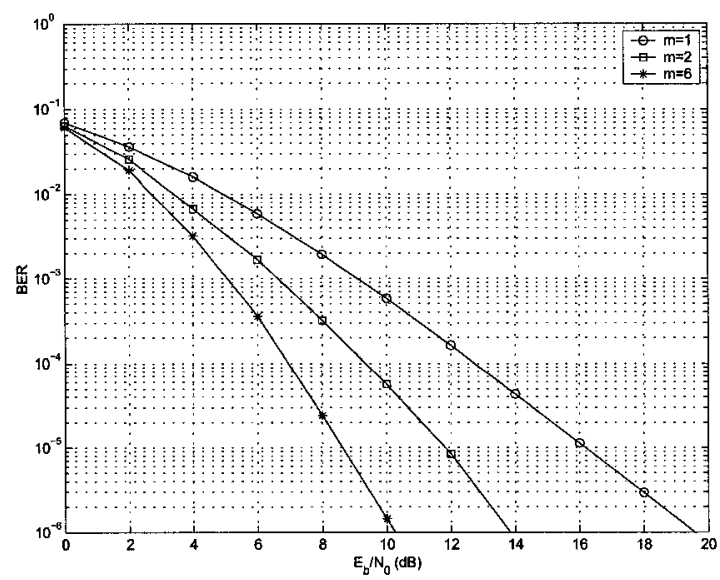

(a) $N_{R}=4, L=1$

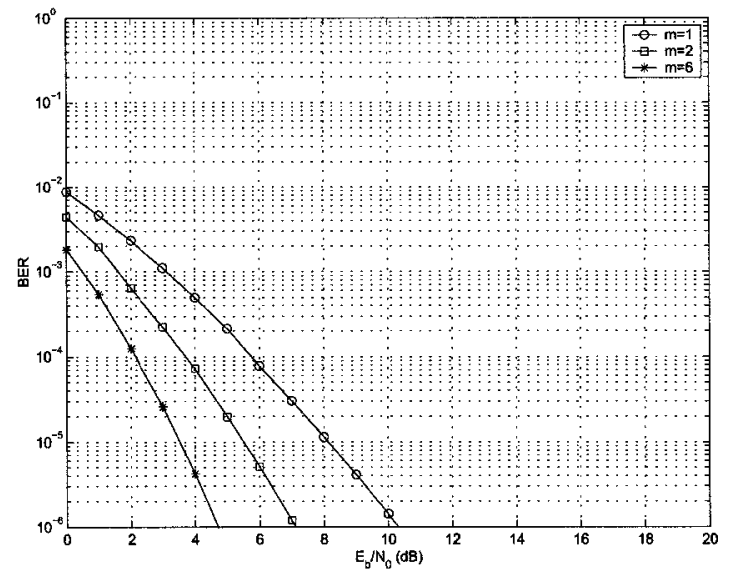

(c) $N_{R}=4, L=4$

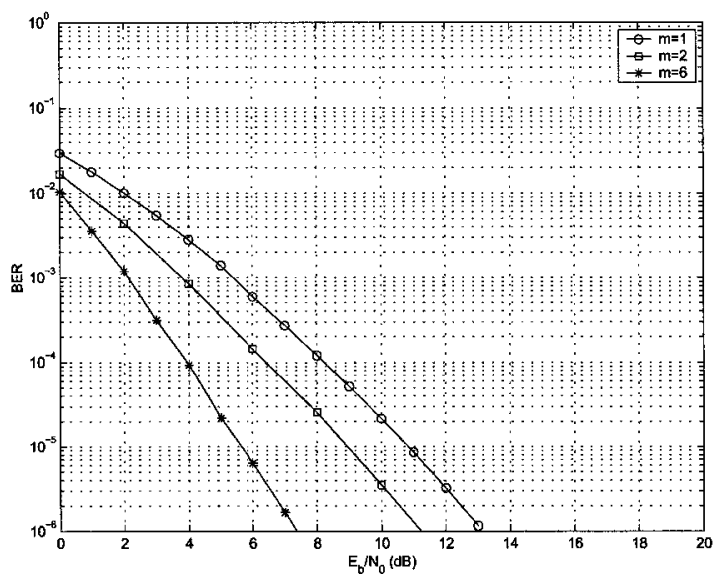

(b) $N_{R}=4, L=2$

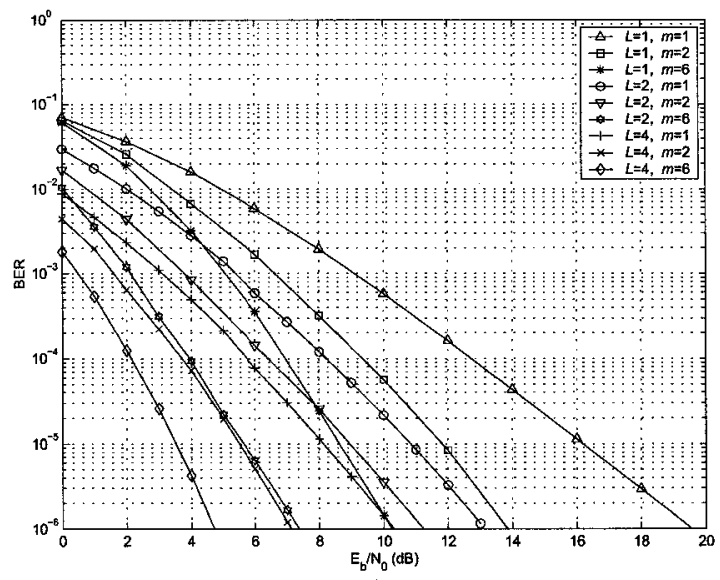

(d) $N_{R}=4, L=1,2,4$

Figure 4.15: BER performance of MRC-based multi-antenna TDF relay in Nakagami fading, $m=1,2,6$ and $N_{R}=4$. 
1). Figure 4.16 (a), (b), (c), and (d) show that MRC-based relay detection could yield no more than $2 \mathrm{~dB}$ gain over the $\mathrm{SC}-$ based. The maximum gain is recorded for a large system configuration, specifically with $N_{R}=4, L=4$. For a smaller number of relays (say $N_{R}=2$ ), the gain considerably shrinks. Furthermore, it is observed that though MRC-based is about $2 \mathrm{~dB}$ superior to the SC-based detection, the former is at the expense of a huge system cost in the $N_{R}=4, L=4$ system architecture. For instance, while the SC-based requires only 4 detection chains (one at each relay station), the MRC requires 16 (four at each relay station). The low SNR gain cannot offset the cost disadvantage incurred by using MRC. Therefore, SC offers a good tradeoff between cost and performance. Finally, for higher values of the Nakagami parameter $(m>1)$ MRC still records gains over the SC-based detection but these gains are too minimal to justify for the cost involved. This, further, shifts the balance in favor of the SC-based detection at relays.

The SC-based detection for different system configurations is compared (Table 4.1). Again, SNR required for an error rate $10^{-4}$ for different parameters, $L$ and $m$ is considered.

The comparison begins with $\left(N_{R}=4, L=1\right),\left(N_{R}=4, L=2\right)$, and $\left(N_{R}=2, L=4\right)$. In the case of $N_{R}=4, L=1$ and $N_{R}=4, L=2$, four detection chains are required but $N_{R}=4, L=2$ requires extra four inexpensive antennas and switching mechanisms. It is observed that in Rayleigh fading environments, a gain as high as $3.8 \mathrm{~dB}$ is obtained with $N_{R}=4, L=2$ over the $N_{R}=4, L=1$ configuration; this gain drops to $1.6 \mathrm{~dB}$ for $m=2$. When the $N_{R}=2, L=4$ system configuration is employed in place of $N_{R}=4, L=2$, it is observed that degradation of 1.6 and $0.2 \mathrm{~dB}$, for $m=1$ and $m=2$, respectively, are incurred. However, two detection chains are required for $\left(N_{R}=2, L=4\right)$ as compared to four that are required for $N_{R}=4, L=2$. Furthermore, Figure 4.16 and Table 4.1 indicate that deploying two relays each with two antennas $N_{R}=2, L=2$ with $\mathrm{SC}$ at the relay yields almost the same performance as deploying four relays each with one antenna $N_{R}=4, L=1$ (see Figure 4.15). It is worth mentioning that two detection resources are required in the 


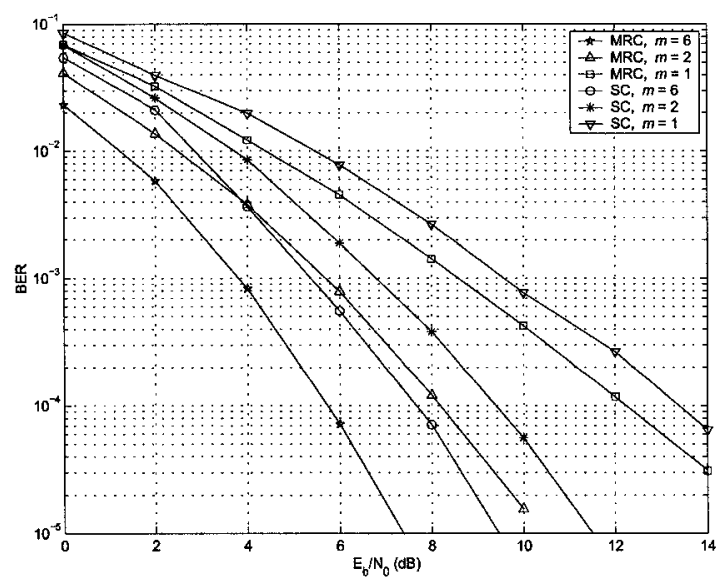

(a) $N_{R}=2, L=2$

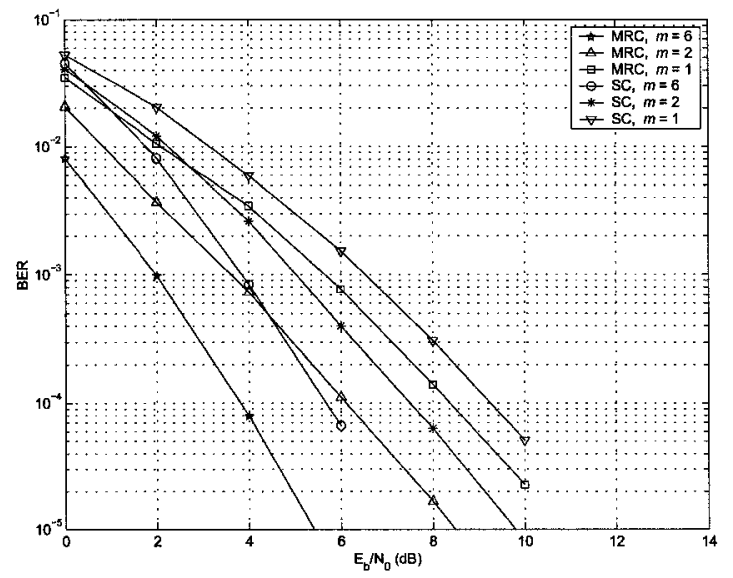

(c) $N_{R}=4, L=2$

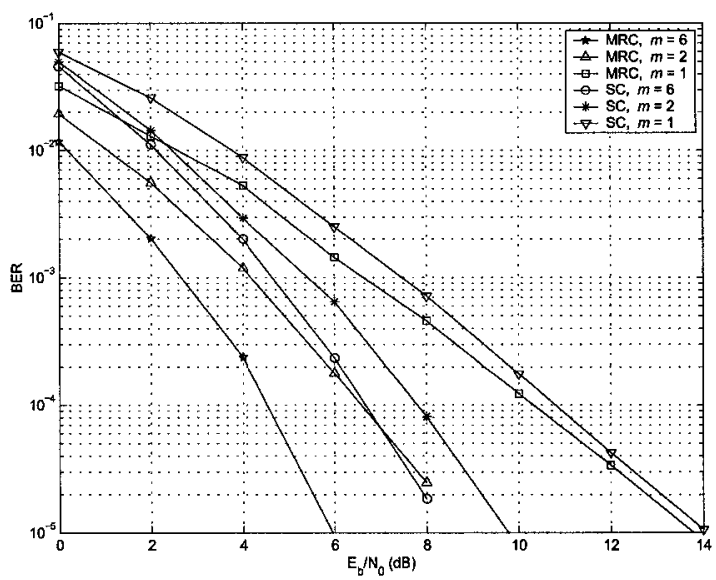

(b) $N_{R}=2, L=4$

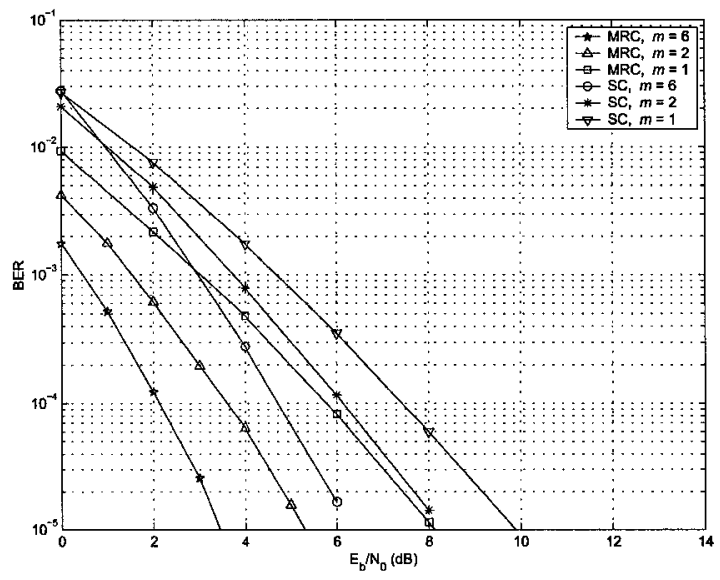

(d) $N_{R}=4, L=4$

Figure 4.16: Performance comparison of MRC-based vs SC-based multi-antenna TDF relay in Nakagami fading, $m=1,2,6$. 
Table 4.1: MRC-based and SC-based Relay Detection: System SNR Comparison at BER = $10^{-4}$

\begin{tabular}{||c|c|c|c|c||}
\hline \hline MRC-based & \multicolumn{2}{|c|}{$N_{R}=2$} & \multicolumn{2}{c||}{$N_{R}=4$} \\
\hline \# of antennas $L$ & $m=1$ & $m=2$ & $m=1$ & $m=2$ \\
\hline 1 & $18.0 \mathrm{~dB}$ & $11.5 \mathrm{~dB}$ & $13.0 \mathrm{~dB}$ & $9.2 \mathrm{~dB}$ \\
\hline 2 & $12.0 \mathrm{~dB}$ & $8.0 \mathrm{~dB}$ & $8.0 \mathrm{~dB}$ & $6.4 \mathrm{~dB}$ \\
\hline 4 & $10.4 \mathrm{~dB}$ & $6.8 \mathrm{~dB}$ & $5.8 \mathrm{~dB}$ & $3.8 \mathrm{~dB}$ \\
\hline SC-based & \multicolumn{2}{|c|}{$N_{R}=2$} & \multicolumn{2}{|c|}{$N_{R}=4$} \\
\hline \# of antennas $L$ & $m=1$ & $m=2$ & $m=1$ & $m=2$ \\
\hline 1 & $18.0 \mathrm{~dB}$ & $11.5 \mathrm{~dB}$ & $13.0 \mathrm{~dB}$ & $9.2 \mathrm{~dB}$ \\
\hline 2 & $13.2 \mathrm{~dB}$ & $9.2 \mathrm{~dB}$ & $9.2 \mathrm{~dB}$ & $7.6 \mathrm{~dB}$ \\
\hline 4 & $10.8 \mathrm{~dB}$ & $7.8 \mathrm{~dB}$ & $7.6 \mathrm{~dB}$ & $6.1 \mathrm{~dB}$ \\
\hline \hline
\end{tabular}

$N_{R}=2, L=2$ case whereas the $N_{R}=4, L=1$ case necessitates four detection resources. Besides, the resource required for deploying extra relays is not comparable to microdiversity antenna elements. Therefore, deploying microdiversity at relays may result in considerable savings in the number of relays deployed in a given area.

\subsection{On the System Complexity and Bandwidth Preser- vation}

A multi-relay scheme employing repetition-based protocol could require enormous utilization of bandwidth since each relay requires its own sub-channel, orthogonal time slots in TDMA or different frequency slots in FDMA applications to transmit the same signal. For a large number of relays, the bandwidth efficiency of such schemes might intolerably be low to the extent that the cost could by far outweigh the advantages of this protocol. To avoid such an 
inefficient use of bandwidth, possible modifications on the conventional relays are discussed. The multiple relay system could employ simulcast techniques, where in the second hop the relay set (those with SNR greater than the threshold) simultaneously transmits their signals after being processed as explained below.

A possible signal processing at the relay could be the use of a finite impulse response (FIR) to filter the input symbols prior to performing a linear-type digital modulation scheme like MPSK, MQAM, and others. The impulse responses of these relay-embedded filters are different from one relay station to another. However, they are designed to meet the necessary conditions to exploit the optimum diversity gain of MRC; a form of orthogonality can be achieved, not in terms of bandwidth hungry time or frequency slots but in terms of filter impulse responses. This approach known as a transmit modulation diversity is shown to be a fraction of $1 \mathrm{~dB}$ inferior to the classical MRC receive diversity [76]. More details on pre-coding filter and equalizer design procedures are provided in $[76,77]$.

Moreover, another possible strategy is the coherent cooperative transmissions from multiple adjacent nodes proposed in [78], where prior to transmissions of the signals, these multiple nodes adjust their transmission characteristics, for example, phases and symbol timings, so that their signals add up coherently at the destination. In fading channels, however, this adjustment is based on the a priori knowledge of the forward channel which can be obtained through feedback from the BS in TDD-based systems.

These modifications (complexities) of the relays could be considered in the deployment of the multi-antenna multi-relay schemes in order to avoid bandwidth expansion involved in the repetition or round-robin transmission protocols. The amount of complexity of relays would depend on how much bandwidth the system designer is prepared to exchange for lower system complexity 


\subsection{Chapter Conclusion}

This chapter investigates the cooperative diversity achieved when multiple relays and source engage into cooperation in two-hop wireless networks. Since space, cost, and signal processing constraints prohibit the use of a large number of antennas at mobile terminals, the future wireless communication systems can exploit the promises associated with multi-antenna techniques by engaging multiple relays in cooperation to mimic a large array of antennas.

Infrastructure-based fixed relays may have the capability to carry multiple antennas, in contrast to mobile terminals. Therefore, the chapter examines multi-antenna aspects of the cooperative two-hop networks. Threshold maximal ratio combining and threshold selection combining techniques to diversity process relay signals are analyzed. These techniques provide enhancements in the error performance of the system over the conventional relaying. From end-to-end performance perspective, it is derived that for a given performance requirement, the multiple antennas at relays can significantly reduce the number of relays required in a network area, with a considerable impact on the system deployment investment. The selection combining-based relaying requires a single RF detection chain similar to the conventional single antenna relays. Therefore, the threshold selection combining emerges as an excellent candidate in terms of a performance-complexity tradeoff.

An important conclusion that can be derived is that two antennas are enough to provide the maximum diversity order, moreover, any additional antenna will only increase the antenna gains. Furthermore, it is also found that for large $L$, a threshold should be avoided. However,

for small $L$, heuristic means to find the optimum choice for Rayleigh fading are used. A rigorous treatment of optimum threshold for decode-and-forward protocol is addressed in [59] for a simple set-up. 


\section{Chapter 5}

\section{Asymmetric Relay Networks}

The commonly assumed symmetry in multiple relay channels is not realistic. Therefore, this chapter investigates asymmetric relay deployment where the links of cooperating nodes to destination could experience unequal signal strength. The cooperative strategy described earlier is used as a foundation.

A network with an arbitrary number of relays with arbitrary positions is considered. Such set-ups are referred to as asymmetric networks in contrast to the symmetric ones which are usually used in the literature [63]. In the symmetric networks, the multiple relays are either treated as being at the same location as the source or that all the relays are at the same location and midway between the source and destination. Although, this assumption is convenient for analysis purposes, is not suitable for many practical systems. Furthermore, the treatment in this chapter assumes that these relays could experience different channel distributions to the destination. Therefore, it is easy to investigate different network topologies which is not the case with other research. Moreover, the end-to-end error performance formulation in the previous chapter and [89] can be applied to this work with a few modifications.

In addition, the analysis represents, in certain scenarios, a tight bound for the E2E error rate of the two-hop network, for example, when a relay adopts threshold decode-and-forward strategy and/or multi-antenna processing to improve the reliability of its detection. 


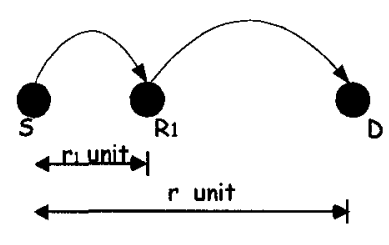

(a)

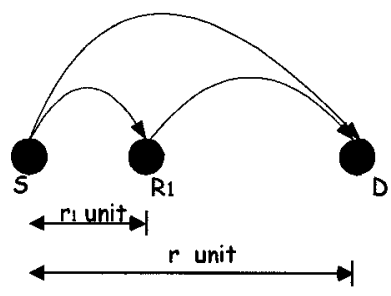

(b)

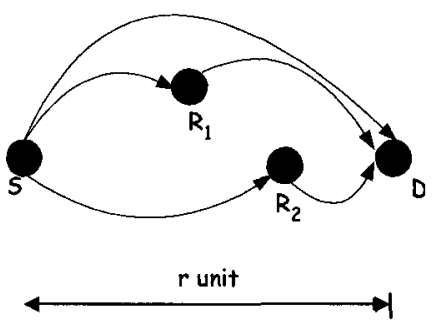

(c)

Figure 5.1: Two-hop network configurations (a) conventional relaying, (b) cooperative network, (c) cooperative network (parallel relays).

In an asymmetric network scenario, all links can have different average SNRs. These average SNRs are represented as $\bar{\gamma}_{i}, i=1, \cdots, N_{R}+1$, where $N_{R}$ denotes the number of relays. Considering the two-hop network which is represented by the simple topology in Figure 5.1, and using the model in [90], the average SNR between a node located at $\left(\mathrm{x}_{r}, \mathrm{y}_{r}\right)$ and the destination located at $(\mathrm{x}, 0)$ can be expressed as $\bar{\gamma}_{r}=\bar{\gamma}\left[\frac{\mathrm{x}}{\sqrt{\left(\mathrm{x}-\mathrm{x}_{r}\right)^{2}+\mathrm{y}_{r}^{2}}}\right]^{\kappa}$, where $\kappa$ is the path loss exponent, and $\bar{\gamma}$ is the average SNR for a single-hop source-destination link. For the purposes of simplicity, all the receiving nodes are assumed to have the same noise power. The total power of the transmitting nodes is the same as the transmit power of the reference single hop transmission.

In the following section of the chapter, the cooperative error performance component for the asymmetric network is discussed. This constitutes the second phase of the two-hop scheme. It is demonstrated that in certain situations (such as, when S-R links are highly reliable), the derived expressions can be used as a quick means of evaluating the E2E network performance. 


\subsection{Analysis}

The analysis begins with the probability density function (PDF) of the output SNR, $\gamma$, of the destination receiver that uses MRC to process the signals received via the $N_{R}$ parallel relays in cooperation with the source. The characteristic function (CF) approach [29] is employed. However, the moment generating function approach can also be used [92]. In this formulation and subsequent treatments, the destination is assumed to receive signals from all relays. This implies that with the TDF protocol $[58,89]$, the received SNR at the relays is always above the decoding threshold or that the decoded block always passes the CRC test.

It should be recalled that $\gamma=\sum_{i=1}^{N_{R}+1} \gamma_{i}$ and the Nakagami PDF of $\gamma_{i}$ is given as

$$
\begin{aligned}
& p_{\gamma_{i}}\left(\gamma_{i}, m_{i}\right)=\frac{m_{i}^{m_{i}} \gamma_{i}^{m_{i}-1}}{\bar{\gamma}_{i}^{m_{i}} \Gamma\left[m_{i}\right]} \exp \left(-\frac{m_{i} \gamma_{i}}{\bar{\gamma}_{i}}\right), \\
& \gamma_{i} \geq 0, m_{i} \geq 1 / 2
\end{aligned}
$$

The CF of a random variable $\gamma_{i}$ is defined as $E\left(e^{j v \gamma_{i}}\right) \equiv \psi(j v)=\int_{-\infty}^{\infty} e^{j v \gamma_{i}} p\left(\gamma_{i}\right) d \gamma_{i}[29$, p. 35, (2-1-71)], and with (5.1), the CF of a single branch SNR, $\gamma_{i}$, with Nakagami fading $m_{i}$ can be expressed as $\psi_{\gamma_{i}}(j v)=\frac{1}{\left(1-j v \frac{\gamma_{i}}{m_{i}}\right)^{m_{i}}}$. Since $\gamma$ is the sum of $N_{R}+1$ statistically independent

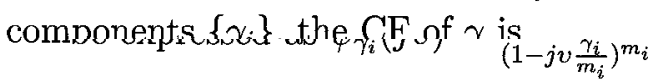

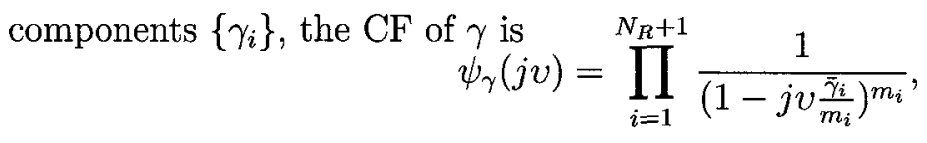

and, therefore, the PDF of $\gamma$ can be obtained by taking the inverse Fourier transform of (5.2). This can be achieved by resolving (5.2) into its partial fractions. Afterwards, by employing [91, pp. 168, (4.43)-(4.44)] for multiple repeated roots (at $j v=\omega=\frac{m_{i}}{\bar{\gamma}_{i}}$ ) of order $m_{i}$, the PDF can be expressed as

$$
p(\gamma)=\sum_{i=1}^{N_{R}+1} \sum_{k=1}^{m_{i}} \varrho_{i, k}\left(\frac{m_{i}}{\bar{\gamma}_{i}}\right)^{k} \frac{\gamma^{k-1}}{\Gamma[k]} \exp \left(-\frac{m_{i} \gamma}{\bar{\gamma}_{i}}\right)
$$


where

$$
\begin{aligned}
\varrho_{i, k} & =\frac{1}{\left(m_{i}-k\right) !\left(-\frac{\bar{\gamma}_{i}}{m_{i}}\right)^{m_{i}-k}} \\
& \times\left\{\frac{\partial^{m_{i}-k}}{\partial \omega^{m_{i}-k}}\left(1-\frac{\bar{\gamma}_{i}}{m_{i}} \omega\right)^{m_{i}} \psi_{\gamma}(\omega)\right\}_{\omega=\frac{m_{i}}{\bar{\gamma}_{i}}} .
\end{aligned}
$$

The error performance of a number of modulation schemes can be evaluated using the PDF given above. For instance, when binary phase shift keying (BPSK) modulation format is adopted on all of the links, the cooperative average bit error rate (BER) can be expressed in the following closed-form.

$$
\begin{aligned}
P_{e} & =\frac{1}{2} \int_{0}^{\infty} \operatorname{erfc}(\sqrt{\gamma}) p(\gamma) d \gamma \\
& =\frac{1}{2} \sum_{i=1}^{N_{R}+1} \sum_{k=1}^{m_{i}} \varrho_{i, k}\left(\frac{m_{i}}{\bar{\gamma}_{i}}\right)^{k} \int_{0}^{\infty} \operatorname{erfc}(\sqrt{\gamma}) \frac{\gamma^{k-1}}{\Gamma[k]} \exp \left(-\frac{m_{i} \gamma}{\bar{\gamma}_{i}}\right) d \gamma
\end{aligned}
$$

After evaluating the integral part in the above expression, $P_{e}$ becomes,

$$
P_{e}=\frac{1}{2} \sum_{i=1}^{N_{R}+1} \sum_{k=1}^{m_{i}} \varrho_{i, k}\left(\frac{m_{i}}{\bar{\gamma}_{i}}\right)^{k} \frac{\Gamma[k+1 / 2]}{\sqrt{\pi} \Gamma[k+1]}{ }_{2} F_{1}\left[k, k+1 / 2 ; k+1 ;-m_{i} / \bar{\gamma}_{i}\right],
$$

where ${ }_{2} F_{1}[\cdot, \cdot ; \cdot ; \cdot]$ is the hypergeometric function $[80]$.

Further simplification can be performed in (5.6) by using [80] to express ${ }_{2} F_{1}[k, k+1 / 2 ; k+$ $\left.1 ; \frac{-m_{i}}{\bar{\gamma}_{i}}\right]$ as $\left(\frac{\bar{\gamma}_{i}}{\bar{\gamma}_{i}+m_{i}}\right)_{2}^{k} F_{1}\left[k, 1 / 2 ; k+1 ; \frac{m_{i}}{\bar{\gamma}_{i}+m_{i}}\right]$. Finally using [80], the following closed-form expression for $P_{e}$ is obtained as

$$
P_{e}=\sum_{i=1}^{N_{R}+1} \sum_{k=1}^{m_{i}} \varrho_{i, k} \frac{\Gamma[k+1 / 2]}{2 \sqrt{\pi} \Gamma[k]} B_{x_{i}}[k, 1 / 2]
$$

where $x_{i}=\frac{m_{i}}{\bar{\gamma}_{i}+m_{i}}$ and $B_{x}[\cdot, \cdot]$ is the incomplete beta function.

The expression given in (5.7) can be employed in a number of scenarios to investigate the performance of two-hop communication networks. Section 5.2 discusses some scenarios where the derived expression could be used for evaluating the system performance. 


\subsection{Scenarios}

The error performance expression (5.7) can be employed in a number of network scenarios:

- In delay-tolerant and low transmit power applications such as mobile infostation network [85]. An example of such an application is the following. A source node wants to deliver certain packets to a destination node. This source could identify two intermediate nodes (e.g., 1 and 2) that pass by and the source relays its packets to them. These relay nodes, however, are not the destination of these packets. When each of the relay nodes ( 1 and 2 ) approaches the destination, they, on behalf of the source node, forward the packet to the destination to complete the second hop of the relaying. Note that due to mobility, the relay-destination channels are not expected to be the same. In this scenario, the expression (5.7) represents a good estimate of the system performance since the first hop is conducted while the source is favorably close to the relay nodes.

- The expression represents a good estimate of the performance of a two-hop cooperative relay network when powerful codes or other error management schemes are employed to ensure reliable source-relay channel, and thereby mitigating relay error propagation. In this case, (5.7) represents a tight bound for the system performance. In the numerical examples section, it is demonstrated that the expression is a tight bound for the system performance of TDF relaying protocol. For instance, the E2E error performance of this protocol $P_{\mathrm{E} 2 \mathrm{E}, \mathrm{TDF}}$ can be lower bounded as $P_{\mathrm{E} 2 \mathrm{E}, \mathrm{TDF}} \geq P_{\boldsymbol{e}}$. The aforementioned examples are scenarios where (5.7) can be used as a stand-alone expression. However, complementing the work in [89], appropriate modifications of their analytical results for symmetric networks can be performed in conjunction with (5.7); the exact E2E error performance of the asymmetric networks can be obtained. Such modifications are straightforward. 


\subsection{Numerical Illustration}

Four cases have been considered to illustrate the performance of the asymmetric relay channels. This section includes the simulation results and a demonstration of the use of (5.7).

Cases I and II have one and two cooperating relays, respectively, and all the links are assumed to experience Rayleigh fading $(m=1)$. Cases III and IV have also one and two cooperating relays, respectively, but the links are of mixed modes described as follows. Case III consists of a cooperative relay, positioned to have a line-of-sight (LOS) link to destination. For this purpose, a Ricean fading model with a $\mathcal{K}$ factor of $3.83 \mathrm{~dB}$ is used, it is equivalent to the Nakagami parameter $m=2$. Case IV considers two cooperative relays both with LOS links to destination with Ricean $\mathcal{K}=3.83 \mathrm{~dB}$.

The source-destination (S-D) link is denoted as channel 1 , with parameters $m_{1}$ and $\bar{\gamma}_{1}$. The $m$ parameter and average SNR for the relay-destination (R-D) links (which constitute the second hop) are $m_{i}$ and $\bar{\gamma}_{i}$, respectively, $i=2,3$. The factors $\varrho_{i, k}$ required for evaluating (5.7) can be computed as follows:

Note that in the following illustrations $\gamma_{i} m_{k} \neq \gamma_{k} m_{i}$.

- Case I. One cooperating relay, with $m_{1}=1$ and $m_{2}=1$. The following factors have to be computed

$$
\varrho_{1,1}=\frac{\bar{\gamma}_{1}}{\bar{\gamma}_{1}-\bar{\gamma}_{2}} \text { and } \varrho_{2,1}=\frac{\bar{\gamma}_{2}}{\bar{\gamma}_{2}-\bar{\gamma}_{1}}
$$

and $\gamma_{i} \neq \gamma_{k}$ for $i \neq k$

- Case II. Two cooperating relays, with $m_{1}=1, m_{2}=1$, and $m_{3}=1$. Then,

$$
\begin{aligned}
& \varrho_{1,1}=\frac{1}{\left(1-\frac{\bar{\gamma}_{2}}{\bar{\gamma}_{1}}\right)\left(1-\frac{\bar{\gamma}_{3}}{\bar{\gamma}_{1}}\right)}, \\
& \varrho_{2,1}=\frac{1}{\left(1-\frac{\bar{\gamma}_{1}}{\bar{\gamma}_{2}}\right)\left(1-\frac{\bar{\gamma}_{3}}{\bar{\gamma}_{2}}\right)}, \\
& \varrho_{3,1}=\frac{1}{\left(1-\frac{\bar{\gamma}_{2}}{\bar{\gamma}_{3}}\right)\left(1-\frac{\bar{\gamma}_{1}}{\bar{\gamma}_{3}}\right)} .
\end{aligned}
$$


- Case III. One cooperative relay, with $m_{1}=1$ and $m_{2}=2$. We need to evaluate $\varrho_{1,1}, \varrho_{2,1}$, and $\varrho_{2,2}$.

$$
\varrho_{1,1}=\frac{1}{\left(1-\frac{\bar{\gamma}_{2}}{2 \bar{\gamma}_{1}}\right)^{2}}, \quad \varrho_{2,1}=\frac{-2 \frac{\bar{\gamma}_{1}}{\bar{\gamma}_{2}}}{\left(1-\frac{2 \bar{\gamma}_{1}}{\bar{\gamma}_{2}}\right)^{2}}, \text { and } \varrho_{2,2}=\frac{1}{\left(1-\frac{2 \bar{\gamma}_{1}}{\bar{\gamma}_{2}}\right)} .
$$

- Case IV. Two cooperative relays, with $m_{1}=1, m_{2}=2, m_{3}=2$. In this case it is necessary to compute $\varrho_{1,1}, \varrho_{2,1}, \varrho_{2,2}, \varrho_{3,1}$, and $\varrho_{3,2}$. These factors are

$$
\begin{aligned}
\varrho_{1,1} & =\frac{1}{\left(1-\frac{\bar{\gamma}_{2}}{2 \bar{\gamma}_{1}}\right)^{2}\left(1-\frac{\bar{\gamma}_{3}}{2 \bar{\gamma}_{1}}\right)^{2}}, \\
\varrho_{2,1} & =\frac{-2}{\bar{\gamma}_{2}}\left(\frac{\bar{\gamma}_{3}}{\left(1-2 \bar{\gamma}_{1} / \bar{\gamma}_{2}\right)\left(1-\bar{\gamma}_{3} / \bar{\gamma}_{2}\right)^{3}}+\frac{\bar{\gamma}_{1}}{\left(1-2 \bar{\gamma}_{1} / \bar{\gamma}_{2}\right)^{2}\left(1-\bar{\gamma}_{3} / \bar{\gamma}_{2}\right)^{2}}\right), \\
\varrho_{2,2} & =\frac{1}{\left(1-2 \bar{\gamma}_{1} / \bar{\gamma}_{2}\right)\left(1-\bar{\gamma}_{3} / \bar{\gamma}_{2}\right)^{2}}, \\
\varrho_{3,1} & =\frac{-2}{\bar{\gamma}_{3}}\left(\frac{\bar{\gamma}_{2}}{\left(1-2 \bar{\gamma}_{1} / \bar{\gamma}_{3}\right)\left(1-\bar{\gamma}_{2} / \bar{\gamma}_{3}\right)^{3}}+\frac{1}{\left(1-2 \bar{\gamma}_{1} / \bar{\gamma}_{3}\right)^{2}\left(1-\bar{\gamma}_{2} / \bar{\gamma}_{3}\right)^{2}}\right), \\
\text { and } \varrho_{3,2} & =\frac{1}{\left(1-2 \bar{\gamma}_{1} / \bar{\gamma}_{3}\right)\left(1-\bar{\gamma}_{2} / \bar{\gamma}_{3}\right)^{2}} .
\end{aligned}
$$

Figure 5.2 shows the cooperative error performance at the destination using expression (5.7). Different network configurations are considered and BPSK modulation format is employed. A path loss exponent of $\kappa=3$ is used. Since the main interest in this case is the diversity benefit due to cooperative multiple relays, the optimum placements for the relays are not investigated. Provided that the relays are positioned well enough to ensure reliable decoding, the effect of the positions of the relay will only move the curves that are shown, without changing the slope, i.e., the diversity order. Given that, for a single cooperating relay, the relay is located midway between the source and destination in this study. The solid curves represent the case with Rayleigh fading in all links; the dotted ones represent those in which the relays experience LOS links. The fact that all cases have the same total transmit power implies that as the number of relays increases, the power of each transmitting entity decreases. Reducing the transmit power requirement of transmitting nodes could be an efficient system deployment strategy, it has the following advantages: extending battery life-span 


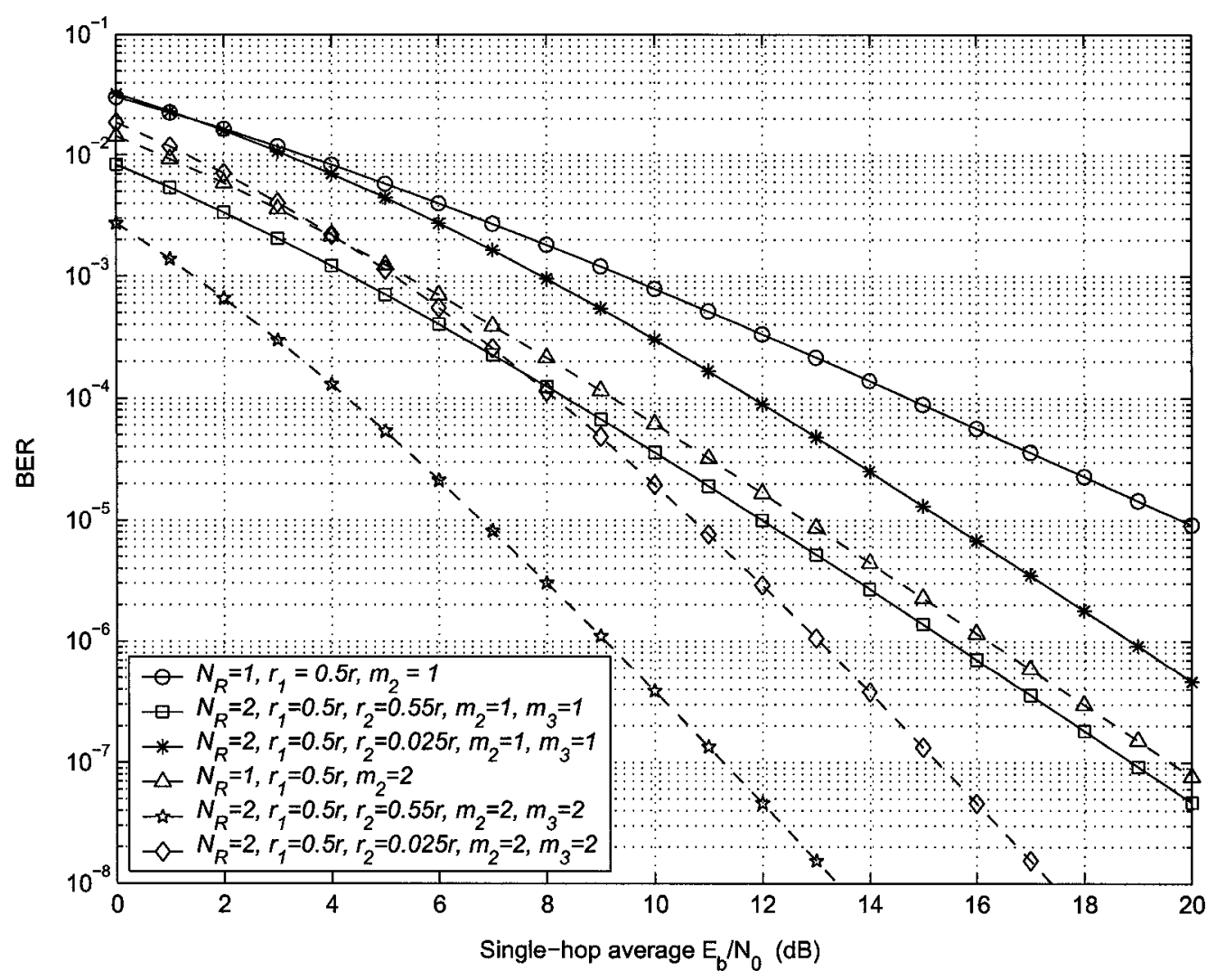

Figure 5.2: Cooperative error performance at the destination of two-hop relay networks. 
for terminals, reducing interference, ensuring lower cost of power amplifier, ultimately leading to inexpensive terminals.

Figure 5.2 indicates that the performance of Case II is better than that of Case I. In addition, the performance of Case IV is superior to that of Case III. This performance superiority is observed from the diversity order measured through the slopes of the performance curves; the curves for Case II and Case IV are steeper than those of Case I and Case III, respectively. The aforementioned gain can be obtained if the detection of the relays is highly reliable, and the relay forwards at all times. In view of this, these performance curves represent the lower bounds of the system performance. For this reason, E2E system performance simulations are conducted to investigate the usefulness of this expression for approximating the system performance. The protocol used in Chapter 4 is employed.

Figure 5.3 shows the bounds for all-Rayleigh links for relay positions $r_{1}=0.5 r$ for $N_{R}=1$, and $r_{1}=0.5 r \& r_{2}=0.55 r$ for $N_{R}=2$. Multiple antennas $(L)$ can be used at the relays to improve the S-R link. The significant impact of the reliability of S-R link is observed when $L=5$ is compared to $L=1$. The bound is tight for a reliable S-R link. The bound is within $2 \mathrm{~dB}$ from the simulated results for $N_{R}=1$ with $L=5$. The difference between the bound and simulated results becomes larger for higher numbers of relays. This is partly due to the fact that the bound assumes very reliable S-R links, whereas simulations do not make such an assumption. It can be, however, inferred from the results that as $N_{R}$ increases, the reliability of S-R links becomes more crucial because of bandwagon effects of decoding errors at the relays. A comparison of the bound with other channel scenarios demonstrates the same performance patterns.

Another scenario, where the bound could be an accurate representation of the system E2E performance, is when the relays are very close to the source. The results for this scenario are shown in Figure 5.4. The relays are placed at locations $r_{1}=0.2 r$ for $N_{R}=1$, and $r_{1}=0.1 r$ $\& r_{2}=0.12 r$ for $N_{R}=2$. The parameter $m=1$ has been used in this example. It is observed 


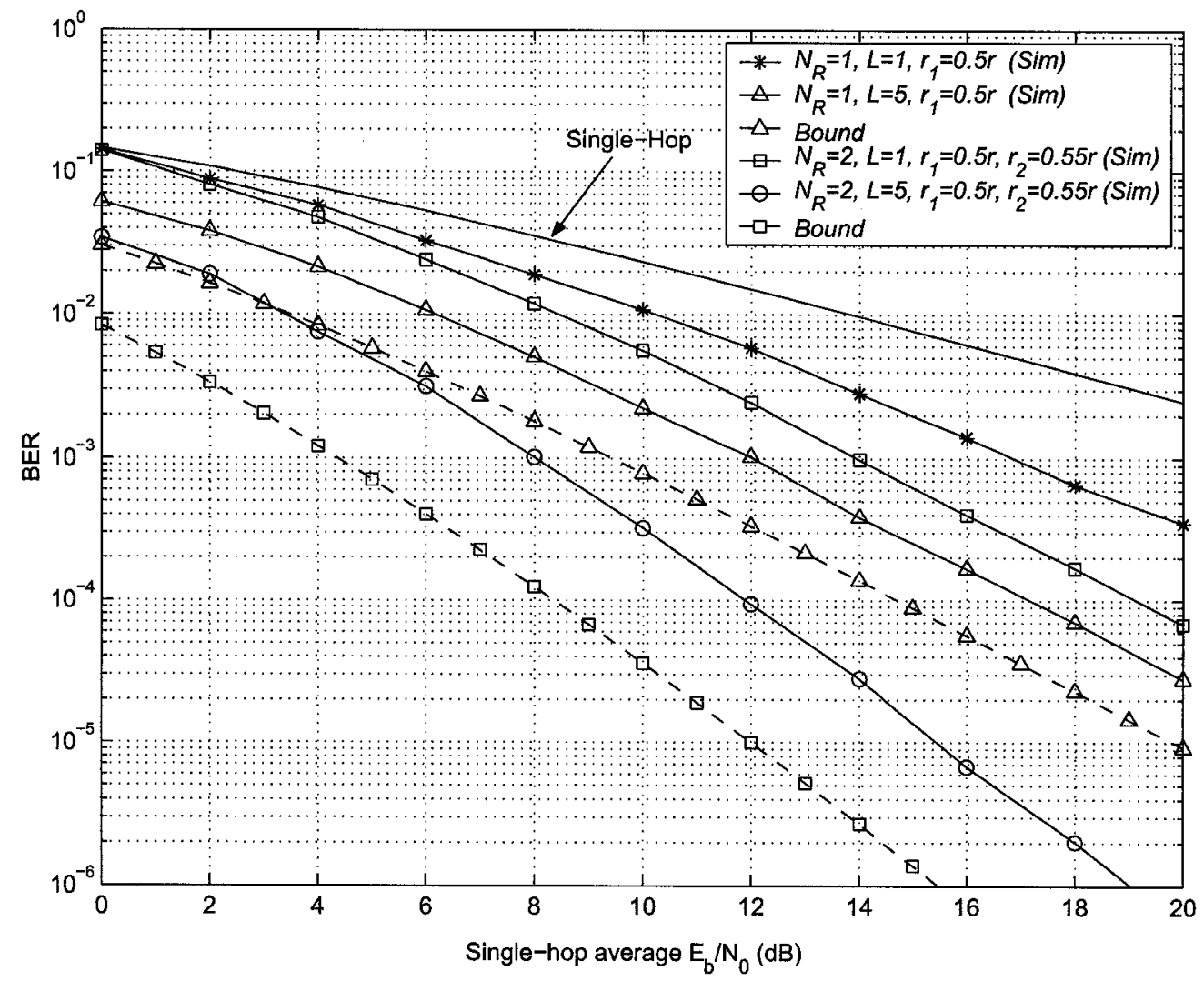

Figure 5.3: Comparison of the bound and simulated end-to-end error performance of relay networks in Rayleigh fading. 


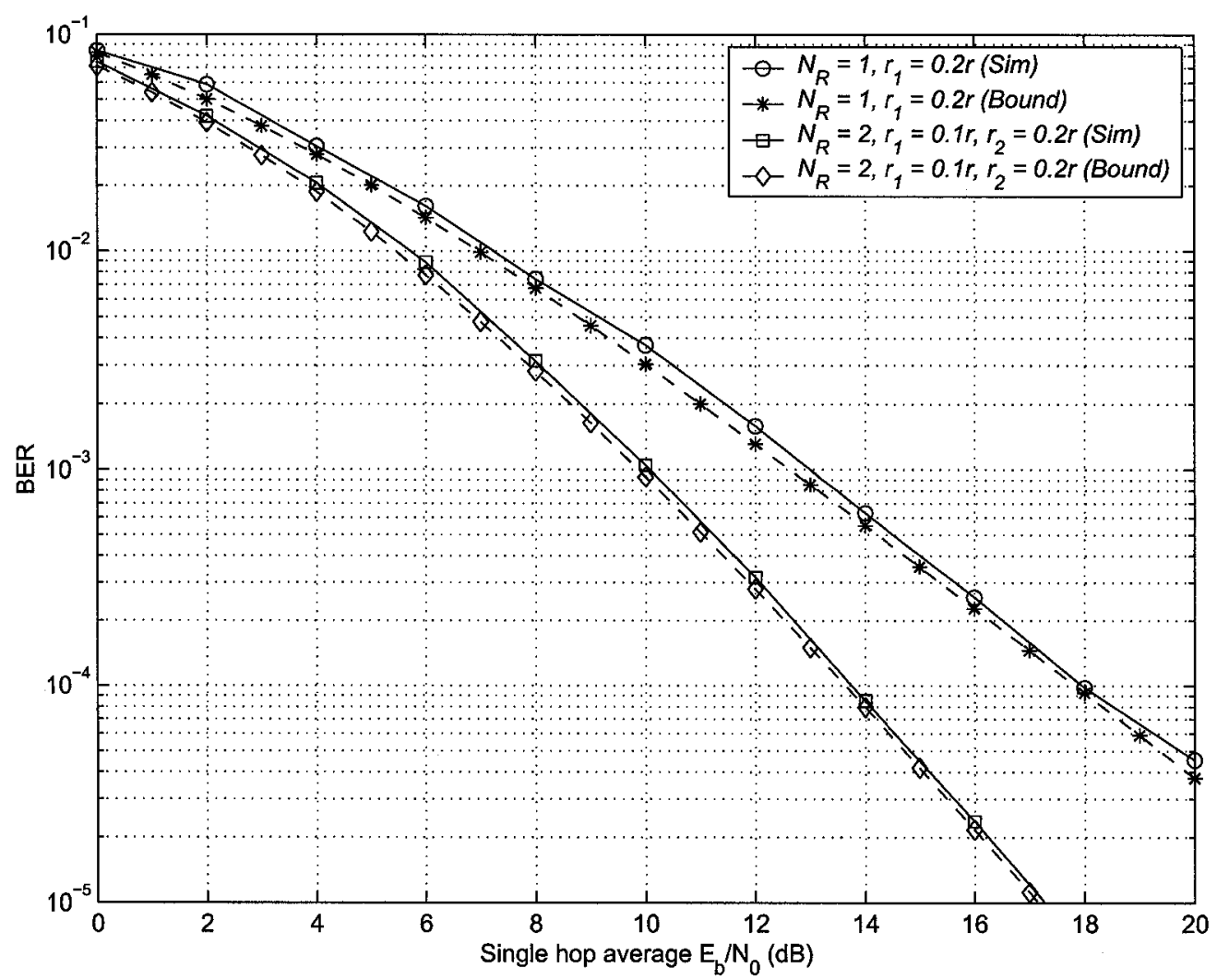

Figure 5.4: Comparison of the bound and simulated end-to-end error performance of relay networks in Rayleigh fading.

that the bound is very tight in this scenario where the relays reliably decode because of their proximity to the source.

Figure 5.5 shows the impact of large relay networks and strategic relay locations in the cooperative scheme. The relay networks that have been considered are $\left\{N_{R}=1, r_{1}=0.5 r\right\}$, $\left\{N_{R}=2, r_{1}=0.5 r, r_{2}=0.55 r\right\}$, and $\left\{N_{R}=3, r_{1}=0.5 r, r_{2}=0.55 r, r_{3}=0.45 r\right\}$. The Nakagami parameter adopted in the link between relay $i$ and the destination is designated as $m_{i+1}(i=1,2,3)$ and the S-D link is designated as $m_{1}$. The Nakagami parameters for the S-R and S-D links are set to one. In general, $m_{i+1}>1(i=1,2,3)$ implies that the relays are placed at locations such that they establish a LOS link to destination whereas in the case of 


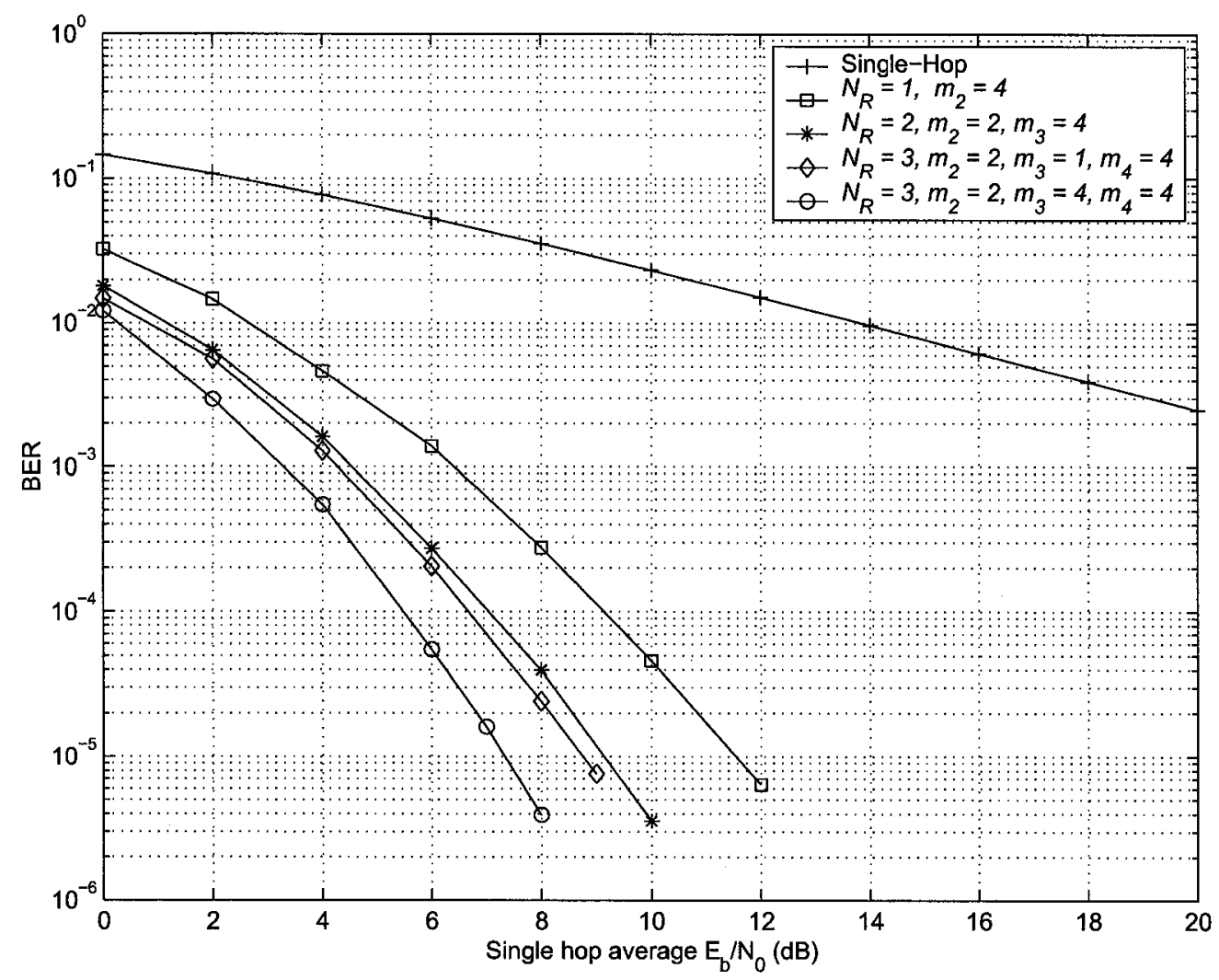

Figure 5.5: End-to-end BER performance of relay networks with different relay locations and $m$-parameter.

S-R links such LOS cannot be guaranteed, this is the justification for the $m=1$ (Rayleigh) in these links. Note that $m=4$ corresponds to a Ricean factor $\mathcal{K}=8.11 \mathrm{~dB}$.

The scheme with one cooperating relay $\left(N_{R}=1\right)$ with a LOS $K=8.11 \mathrm{~dB}$ link to destination $(m=4)$ greatly outperforms the single-hop Rayleigh channel. The curves denoted with asterisk and diamond indicate that in order for additional nodes to be effective they should have reasonably good links to destination. It can be observed that the network configuration with $N_{R}=3, m_{2}=2, m_{3}=1$, and $m_{4}=4$ does not yield any significant performance improvement over that with $N_{R}=2, m_{2}=2$, and $m_{3}=4$. 


\subsection{Chapter Conclusion}

In this chapter the cooperative diversity achieved when multiple relays and source engaged into cooperation in two-hop wireless asymmetric networks is investigated. Normalization of the transmit power is implemented in the multi-relay among the different relay architectures. It, therefore, follows that as the number of relays increases, the power of each transmitting entity decreases. This reduction in the transmit power requirement of nodes and user terminals could be an efficient system deployment strategy; its benefits include: extending battery life-span for terminals, reducing interference, ensuring lower cost of power amplifier, and ultimately leading to inexpensive terminals.

Analytical and simulation results are provided for the two-hop networks for different network configurations of relays and in a wide range of channel distributions using the versatile Nakagami fading channel model. 


\section{Chapter 6}

\section{Fixed Relay-enabled User Cooperation}

\subsection{Motivation}

The explicit user cooperation diversity schemes discussed in $[38,39]$ require that at least two users are in the network and they are willing to cooperate. To sustain such a cooperative scheme, coercion or incentives for the cooperating partners might be needed, which could present implementation challenges. In the conceptual presentation of user cooperation diversity, it is noted that the two cooperating partners achieve mutual instant gain when both users face statistically similar channels to destination. However, in dissimilar channels, the gain for the user that has a better channel is not that instant. In this situation, a "greedy" partner might question the need for cooperation when it faces a good channel to destination. Furthermore, explicit cooperation requires that each cooperating partner detects the data of the other user. Hence, the data of each user would need to be protected from "a malicious" partner it adds another dimension to the security challenges of wireless networks.

From economic point of view, explicit cooperation requires that terminals are modified in order to be able to perform their new tasks, which leads to an increase in terminal complexities. Given that hundreds to thousands of wireless terminals are often served in a network, this involves significant capital investment, thereby imposing capital burden on both service 
providers and end users.

To circumvent the aforementioned problems of user-dependent cooperative diversity, this chapter discusses fixed relay-enabled user cooperation. The scheme operates as follows: two users, ignorant of their cooperation, are engaged in it through two fixed relays. The user terminals do not need to detect each other's data. The benefits derived from this type of cooperation are obvious. First, the privacy of users is not compromised and secondly, sanctioning or rewarding the users for participating in the cooperation is not required as the cooperation is transparent to the users. In addition to these benefits, the fixed relay-enabled user cooperation ensures that user terminals are simple, service is guaranteed, routing is simple, and system deployment is flexible. These are the features that any service provider would like to maintain an unfettered control on.

A typical example of how the flexibility of the proposed scheme can be exploited is the ease with which it can support heterogeneous mode of relaying. It is important to note that most of the previous cooperative relaying faces stiff challenges to operate on two air interfaces since the first and second hops are inherently coupled. This situation is different in the proposed scheme, where the two hops are decoupled. Therefore, the cooperative technique can easily be adapted to support heterogeneous relaying (i.e., two different air interfaces for the relay's receptions and transmissions can be used, in contrast to single air interface in homogeneous relaying). This, for example, implies that the receptions of both the relay and BS could be on two different frequency bands which fits well with the Nortel's mesh network solution. One of these bands could be an unlicensed frequency band. Furthermore, the relays are expected to be synchronized at a symbol level. Since fixed relays are employed, the BS could beacon instruction on the synchronization.

In developing a concept, it is pedagogically sound to work with minimal number of parameters that elicit the salient features of the concept. For this purpose we will mostly use a basic wireless network with two users. 
In the following section, the descriptions of the proposed system is given and some realization examples are discussed. Then, an analytical framework for evaluating the system performance is examined. This is followed by the analysis of the results obtained through simulations and analytical computation.

\subsection{System Descriptions}

In the proposed scheme the signal processing at the relays can be classified into two - spatial division multiple access (SDMA)-based and multiuser detection (MUD)-based processing. In the multi-antenna relay architecture, the relays use the principle of SDMA for detection; for a single user (Figure 6.1) or two partners (Figure 6.2). However, in a single antenna relay architecture, the multiuser detection (MUD) capability is required as shown in a generic form (Figure 6.3); where it is assumed that the users employ some spreading (orthogonal) codes.

In general, after the relays detect the signal in the first hop, they could engage into cooperation using, for example, distributed space-time coding (DSTC)[10]. The scheme using SDMA operates in the following manners which we refer to as realizations I and II.

In realization I, each relay detects the signal of one user by directing a null towards the other (Figure $6.1(\mathrm{a})$ ). To engage the data of the two users in the distributed space-time coding, the signal of the nulled user is obtained by the other relay node by exploiting the broadcast nature of wireless channels. This exchange of user signals is accomplished in the second time slot when each relay node transmits to destination (Figure 6.1 (b)). This imposes full duplex mode of operation on the relay node which has stringent practical implications. The three-slot protocol combines the two-hop relay network with a (virtual) two transmit antenna scheme. For comparison purposes, the following action can be undertaken. First, the sum of the times allocated to the three slots can be made equal to that of the reference scheme. Second, a higher modulation constellation could be employed in the links to allow for the same bandwidth utilization with the reference scheme. 


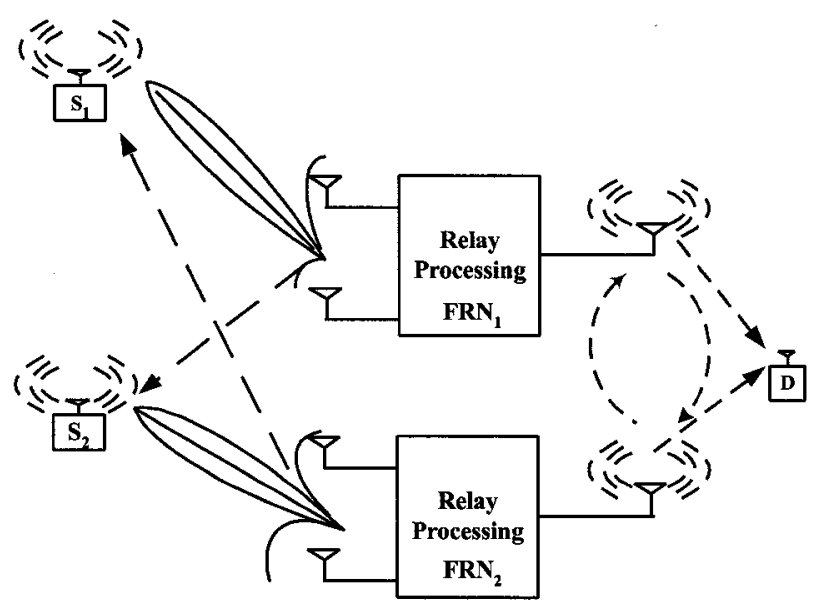

(a)

\begin{tabular}{|c|c|c|}
\hline $\begin{array}{l}S_{1}\left(d_{1}\right) \rightarrow F R N_{1} \\
S_{2}\left(d_{2}\right) \rightarrow F R N_{2}\end{array}$ & $\begin{array}{l}F_{R N}\left(d_{1}\right) \rightarrow D \text { and } F R N_{2} \\
F R N_{2}\left(d_{2}\right) \rightarrow D \text { and } F R N_{1}\end{array}$ & $\begin{array}{l}F_{R N_{1}}\left(d_{2}\right) \rightarrow D \\
F_{2} N_{2}\left(d_{1}\right) \rightarrow D\end{array}$ \\
\hline
\end{tabular}

(b)

Figure 6.1: Fixed relay-enabled user cooperation: Realization I.

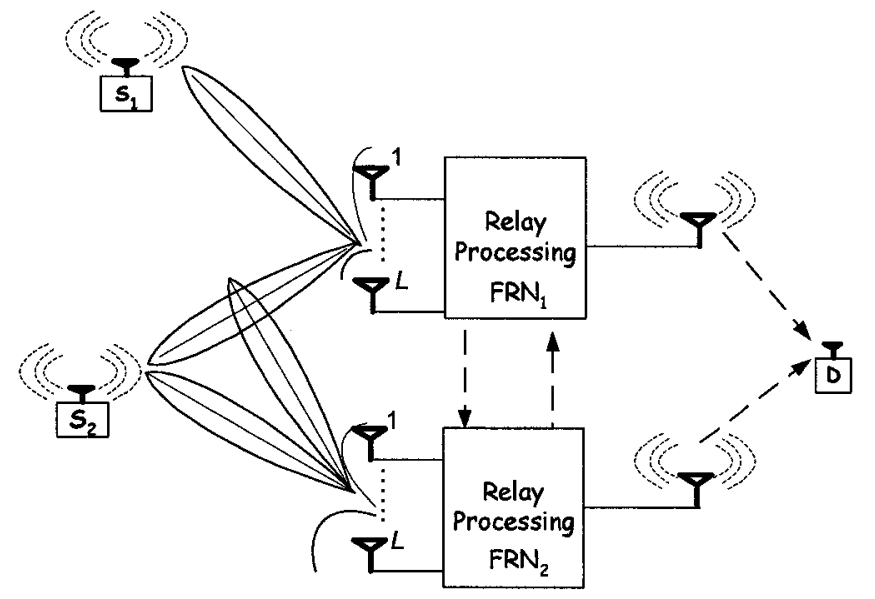

(a)

\begin{tabular}{|c|c|c|}
\hline $\begin{array}{l}S_{1}\left(d_{1}\right) \rightarrow F R N_{1}, F R N_{2} \\
S_{2}\left(d_{2}\right) \rightarrow-\rightarrow F R N_{1}, F R N_{2}\end{array}$ & $\begin{array}{l}\operatorname{FRN}_{1}\left(d_{1}\right) \rightarrow D \\
F R N_{2}\left(d_{2}\right) \cdots D\end{array}$ & $\begin{array}{l}F R N_{1}\left(d_{2}\right) \rightarrow D \\
F^{2} N_{2}\left(d_{1}\right) \rightarrow D\end{array}$ \\
\hline
\end{tabular}

(b)

Figure 6.2: Fixed relay-enabled user cooperation: Realization II. 


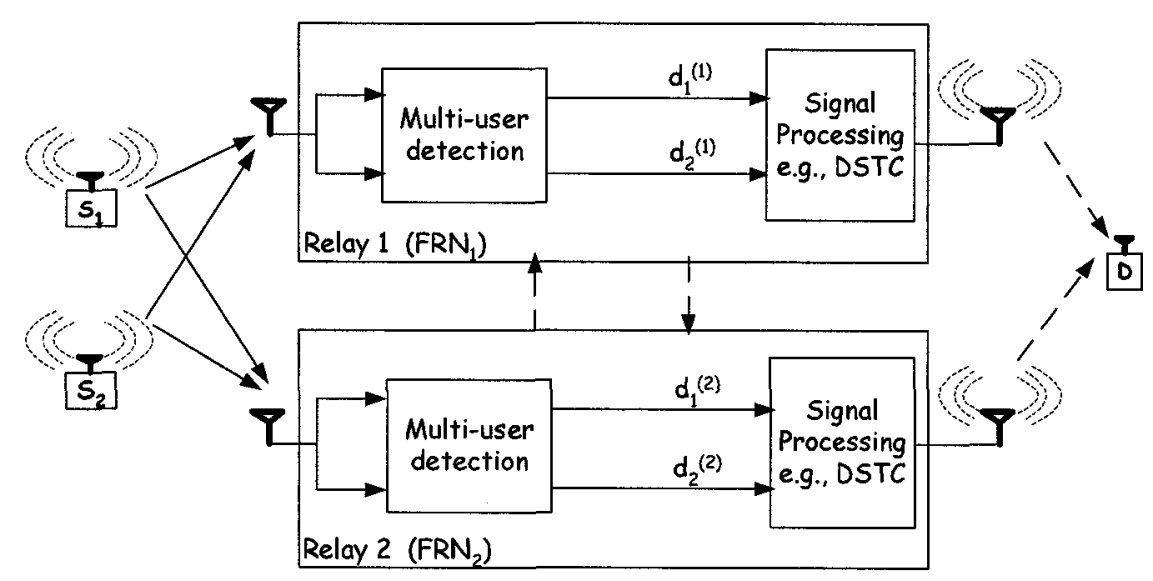

(a)

\begin{tabular}{|c|c|c|}
\hline $\begin{array}{l}S_{1}\left(d_{1}\right) \rightarrow F R N_{1}, F R N_{2} \\
S_{2}\left(d_{2}\right) \rightarrow F R N_{1}, F R N_{2}\end{array}$ & $\begin{array}{l}F^{F N_{1}}\left(d_{1}{ }^{(1)}\right) \rightarrow D D \\
F_{2} N_{2}\left(d_{2}{ }^{(2)}\right) \cdots D\end{array}$ & $\begin{array}{l}F N_{1}\left(d_{2}(1)\right) \rightarrow D \\
F_{2} N_{2}\left(d_{1}{ }^{(2)}\right) \rightarrow D\end{array}$ \\
\hline
\end{tabular}

(b)

Figure 6.3: Fixed relay-enabled user cooperation: Realization III.

The realization II removes the stringent FD requirement at the relay node. However, the relay would need processing power to be able to direct two beams to the desired partners that are to be engaged in cooperation. The third scheme referred to as realization III (Figure 6.3) operates, in principle, in the same way as realization II except in the mode of reception. In this case, the relay-enabled cooperative scheme uses MUD at each relay to make detections of the signals of the two partners. The MUD and spreading are necessary for the single antenna relay architecture. For realization II and III, the system could operate in a mode that can be referred to as a "pre-determined" space-time coding; the relays, in this case, do not need to exchange any instantaneous information as the cooperation is agreed upon a priori. One advantage of this is the removal of the full-duplex requirement on the relays. In this mode, each relay assumes that the signals of the users it detects, have the same reliability as those 
Table 6.1: The encoding and transmission sequence for the distributed space time code

\begin{tabular}{||c|c|c||}
\hline \hline Relay station & FRN $_{1}$ transmits & FRN $_{2}$ transmits \\
\hline \hline time $t+T$ & $\hat{d}_{1}^{(1)}$ & $\hat{d}_{2}^{(2)}$ \\
\hline time $t+2 T$ & $-\hat{d}_{2}^{*(1)}$ & $\hat{d}_{1}^{*(2)}$ \\
\hline \hline
\end{tabular}

detected by the cooperating relay. They simply employ these signals to realize the distributed space time coding. In the implementation of this scheme, however, automatic repeat request in conjunction with cyclic redundancy check might be required to alleviate the problem of performing distributed space-time coding detected data/signal that is in error. Table 6.1 describes the distributed transmissions accomplished by coding across time and space. The datum of user $k$ detected at relay station $i$ is denoted as $d_{k}^{(i)}$.

The price for deploying these cooperation schemes is the increase in the complexity of relays; although this may translate into a cost higher than that of the conventional relays, we argue that the benefits of the proposed scheme outweigh significantly the cost increment. For instance, it is more economical to increase the cost of few relay stations rather than the cost of changing thousands of terminals. This is in addition to a service that is guaranteed through incentive-free, coercion-free, and secured networks.

In the following section, analytical framework for evaluating the performance of realization I is presented. The results obtained from the analysis are discussed and compared with those obtained through simulations.

\subsection{Analysis of Relay-enabled Cooperative Communi- cation Schemes}

We consider the source-relay links with each user carrying a single antenna and the fixed relay equipped with $L$ antennas. It is assumed that the fading rate is slower than the symbol rate. 


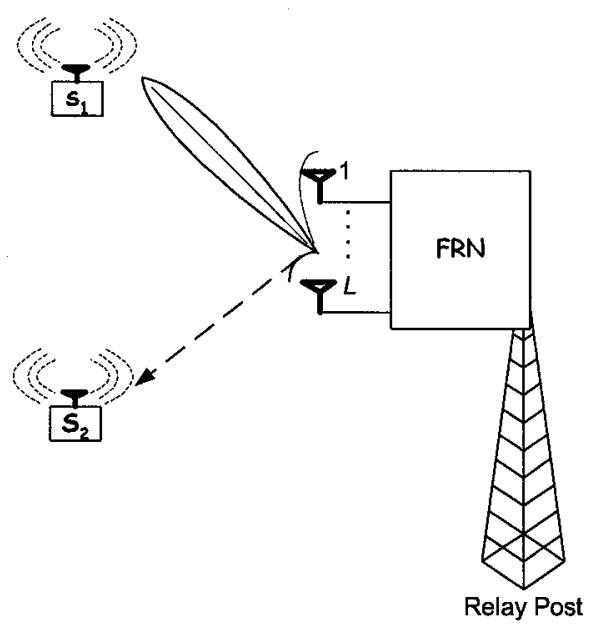

Figure 6.4: Fixed relay node equipped with multi-antenna. The relay uses spatial division multiple access technique to detect a desired user while directing a null to an interfering user (realization I).

After matched filtering, sampling at symbol rate, the base band model for the received signal vector can be expressed as

$$
\mathbf{y}=\sum_{k=0}^{\mathcal{N}_{u}} \boldsymbol{\alpha}_{k} x_{k}+\mathbf{n}_{k}
$$

where $\boldsymbol{\alpha}_{k}$ is the propagation vector for the user $k$, and $\mathbf{n}_{k}$ is the corresponding IID noise. The vectors $\mathbf{y}, \boldsymbol{\alpha}_{k}, \mathbf{n}_{k}$ have a dimension $L$.

The task is to find a set of weight (beamformer) that maximizes the SINR for the desired partner. We will denote this weight vector as $\boldsymbol{\omega}_{\mathrm{opt}}$. However, for general $\boldsymbol{\omega}$, the power at the output of such a combiner can be expressed as ${ }^{1}$

$$
P_{o}=P_{d}+P_{\mathrm{I}+\mathrm{N}}=E_{d}\left|\boldsymbol{\omega}^{*} \boldsymbol{\alpha}_{0}\right|^{2}+\omega^{*} \mathbf{R}_{n n} \boldsymbol{\omega}
$$

where, $P_{d}$ is the desired user power, $P_{\mathrm{I}+\mathrm{N}}$ is the noise plus interferer power, and $\left(^{*}\right)$ is the conjugation transposition operation known as Hermitian operator.

\footnotetext{
${ }^{1}$ Note that $P_{I+N}=E\left[\left|\omega^{*}\left(\sum \boldsymbol{\alpha}_{j} x+\mathbf{n}_{j}\right)\right|^{2}\right]=E\left[\left(\boldsymbol{\omega}^{*}\left(\sum \boldsymbol{\alpha}_{j} x+\mathbf{n}_{j}\right)\right)\left(\left(\sum \boldsymbol{\alpha}_{j}^{*} x^{*}+\mathbf{n}_{j}^{*}\right) \boldsymbol{\omega}\right)\right]=\boldsymbol{\omega}^{*} \mathbf{R}_{n n} \boldsymbol{\omega}$.
} 
The output SINR can represented as

$$
S I N R=\frac{P_{d}}{P_{I+\mathrm{N}}}=\frac{E_{d}\left|\boldsymbol{\omega}^{*} \boldsymbol{\alpha}_{0}\right|^{2}}{\boldsymbol{\omega}^{*} \mathbf{R}_{n n} \boldsymbol{\omega}}
$$

The optimum weight vector (i.e., the beamformer) that yields the maximum SINR can be obtained by minimizing the denominator of (6.3). The minimizing is subject to the constraint $\boldsymbol{\omega}^{*} \boldsymbol{\alpha}_{0}=a$. In general, the $\left|\boldsymbol{\omega}^{*} \boldsymbol{\alpha}_{0}\right|^{2}$ is designed to be unity, usually referred to as distortion-less constraints [87]. The Lagrange approach can be used to perform the minimization. We start by defining a cost function

$$
\begin{aligned}
F(\boldsymbol{\omega}, \boldsymbol{\alpha}) & =\boldsymbol{\omega}^{*} \mathbf{R}_{n n} \boldsymbol{\omega}+2 \operatorname{Re}\left[\chi\left(\boldsymbol{\omega}^{*} \boldsymbol{\alpha}_{0}-a\right)\right] \\
& =\boldsymbol{\omega}^{*} \mathbf{R}_{n n} \boldsymbol{\omega}+\chi\left(\boldsymbol{\omega}^{*} \boldsymbol{\alpha}_{0}-a\right)+\chi^{*}\left(\boldsymbol{\omega}^{*} \boldsymbol{\alpha}_{0}-a^{*}\right)
\end{aligned}
$$

where $\chi$ is Lagrange multiplier.

Taking the gradient with respect to $\boldsymbol{\omega}^{*}$ and setting the result to zero yields

$$
\frac{\partial F(\boldsymbol{\omega}, \boldsymbol{\alpha})}{\partial \boldsymbol{\omega}^{*}}=\mathbf{R}_{n n} \boldsymbol{\omega}_{\text {opt }}^{*}+\chi \boldsymbol{\alpha}_{0}=\mathbf{0}
$$

Therefore,

$$
\boldsymbol{\omega}_{o p t}^{*}=-\chi \mathbf{R}_{n n}^{-1} \boldsymbol{\alpha}_{0}
$$

But $\boldsymbol{\alpha}_{0}^{*} \omega_{\text {opt }}=-\chi \boldsymbol{\alpha}_{0}^{*} \mathbf{R}_{n n}^{-1} \boldsymbol{\alpha}_{0}=a^{*}$. This implies that $\chi=-\left(\boldsymbol{\alpha}_{0}^{*} \mathbf{R}_{n n}^{-1} \boldsymbol{\alpha}_{0}\right)^{-1} a^{*}$. Hence, the optimum weights can be obtained as

$$
\omega_{o p t}=\frac{a^{*} \mathbf{R}_{n n}^{-1} \boldsymbol{\alpha}_{0}}{\boldsymbol{\alpha}_{0}^{*} \mathbf{R}_{n n}^{-1} \boldsymbol{\alpha}_{0}}
$$

Finally, $\boldsymbol{\omega}_{\text {opt }}=\chi \mathbf{R}_{n n}^{-1} \boldsymbol{\alpha}_{0}$. The multiplying constant $(\chi)$ can be dropped without affecting the output SINR. Therefore, the optimum weight can be expressed as

$$
\boldsymbol{\omega}_{\text {opt }}=\mathbf{R}_{n n}^{-1} \boldsymbol{\alpha}_{0}
$$

We can now express the minimum value of $\boldsymbol{\omega}_{o p t}^{*} \mathbf{R}_{n n} \boldsymbol{\omega}_{\text {opt }}=\boldsymbol{\alpha}_{0}^{*} \mathbf{R}_{n n}^{-1} \mathbf{R}_{n n} \mathbf{R}_{n n}^{-1} \boldsymbol{\alpha}_{0}=\boldsymbol{\alpha}_{0}^{*} \mathbf{R}_{n n}^{-1} \boldsymbol{\alpha}_{0}$. Finally, the maximum output SINR, simply denoted as $\gamma$ can be written as

$$
\gamma=E_{d} \frac{\left|\boldsymbol{\omega}_{\mathrm{opt}}^{*} \boldsymbol{\alpha}\right|^{2}}{\boldsymbol{\alpha}_{0}^{*} \mathbf{R}_{n n}^{-1} \boldsymbol{\alpha}_{0}}=E_{d} \frac{\left(\boldsymbol{\alpha}_{0}^{*} \mathbf{R}_{n n}^{-1} \boldsymbol{\alpha}_{0}\right)^{2}}{\boldsymbol{\alpha}_{0}^{*} \mathbf{R}_{n n}^{-1} \boldsymbol{\alpha}_{0}}=E_{d} \boldsymbol{\alpha}_{0}^{*} \mathbf{R}_{n n}^{-1} \boldsymbol{\alpha}_{0}
$$


Since the optimum combiner requires knowledge of the covariance matrix of interference plus noise this parameter is examined. The covariance matrix of the interference plus noise is given by

$$
\begin{aligned}
\mathbf{R}_{n n} & =E\left[\left(\mathbf{y}-\boldsymbol{\alpha}_{0} x_{0}\right)\left(\mathbf{y}-\boldsymbol{\alpha}_{0} x_{0}\right)^{*}\right] \\
& =E\left[\left(\sum_{k=1}^{\mathcal{N}_{u}} \boldsymbol{\alpha}_{k} x_{k}+\mathbf{n}_{k}\right)\left(\sum_{j=1}^{\mathcal{N}_{u}} \boldsymbol{\alpha}_{j} x_{j}+\mathbf{n}_{j}\right)^{*}\right] \\
\mathbf{R}_{n n} & =\sum E\left[\boldsymbol{\alpha}_{k} x_{k} x_{k}^{*} \boldsymbol{\alpha}_{k}^{*}\right]+\sigma^{2} \mathbf{I} .
\end{aligned}
$$

This covariance matrix represents an average performed over a period shorter that the reciprocal of the fading rate. For equal energy signal, $\mathbf{R}_{n n}$ can be expressed as

$$
\mathbf{R}_{n n}=E_{I} \sum_{k=1}^{\mathcal{N}_{u}} E\left[\boldsymbol{\alpha}_{k} \boldsymbol{\alpha}_{k}^{*}\right]+\sigma^{2} \mathbf{I}_{L}=\mathbf{R}_{I}+\mathbf{R}_{n} .
$$

The cooperative scheme identifies two users that are to be engaged in cooperation by the relays. Some simplifying approaches are worth considering. One approach is to consider that at each relay, one strong user (among the $\mathcal{N}_{u}$ users) is interfering and others can be lumped with the system noise. The other approach is that the network consists of two users. In a situation where a partnership cannot be established, it is important that the system has some fall-back mechanisms. This fall-back mechanism could be the multi-antenna multi-relay cooperative discussed in Chapter 4.

For the multi-antenna relays that are investigated here, $L$ is greater than the number of interfering (or dominant) users. In this scenario, the distribution of the interference covariance matrix $\mathbf{R}_{I}$ is pseudo-Wishart's [67]. For the cases just motivated, it is implied that $\mathbf{R}_{I}$ has a single (dominant) non-zero eigen-value $\phi_{1}$ which is real and positive. The remaining $\phi_{i}, i=2, \cdots, L$ are zeros. Thus, the PDF of $\phi_{1}$ of $\mathbf{R}_{I}$ can be expressed using the joint PDF of pseudo-Wishart distribution, which can be simplified to

$$
p\left(\phi_{1}\right)=\frac{\phi_{1}^{L-1}}{(L-1) !} \exp \left(-\phi_{1}\right), \phi_{1}>0 .
$$


Corresponding to each $\phi_{i}$ is an eigen-vector $\boldsymbol{v}_{i}$. That is $\mathbf{R}_{I}=\sum_{i=1}^{L} \phi_{i} \boldsymbol{v} \boldsymbol{v}^{*}$, where $\phi_{i}=0 \mid i>1$. Note that the $\boldsymbol{v}$ 's are orthonormal eigenvectors of the interference correlation matrix. Hence, $\gamma$ in $(6.9)$ can be expressed as $[67,87]$

$$
\gamma=\frac{E_{d}\left|\boldsymbol{\alpha}_{0}^{*} \boldsymbol{v}_{\mathbf{1}}\right|^{2}}{E_{I} \phi_{1}+N_{0}}+\sum_{i=2}^{L} \frac{E_{d}\left|\boldsymbol{\alpha}_{0}^{*} \boldsymbol{v}_{i}\right|^{2}}{N_{0}},
$$

which implies that the conditional characteristic function can written as

$$
\begin{aligned}
\varphi_{\gamma \mid \phi_{1}}(j v) & =\frac{1}{\left(1-j v E_{d} / N_{0}\right)^{L-1}} \times \frac{1}{\left(1-j v \frac{E_{d}}{E_{I} \phi_{1}+N_{0}}\right)} \\
& =\frac{1}{\left(1-j v \bar{\gamma}_{1}\right)^{L-1}} \times \frac{1}{\left(1-j v \bar{\gamma}_{2}\right)}
\end{aligned}
$$

where $\bar{\gamma}_{1}=\frac{E_{d}}{N_{0}}$ and $\bar{\gamma}_{2}=\frac{E_{d}}{E_{I} \phi_{1}+N_{0}}$.

The $\mathrm{CF}$ in (6.14) presents some interesting scenarios. From this equation, the ineffectiveness of one antenna to combat interference is observed. When $L=1$, overall, there is a Rayleigh fading channel with average power modified by the interferer power. On the other hand, when $L>1$, a high power interferer (i.e., $E_{I} \rightarrow \infty$ ) can be nulled out completely and the relay is left with an $L-1$ order of diversity.

The PDF of $\gamma$ conditioned on $\phi_{1}$ can be obtained by taking the inverse Fourier transform of (6.14). This can be achieved by resolving this equation into its partial fractions, thus, this PDF is simplified to

$$
p\left(\gamma \mid \phi_{1}\right)=\sum_{i=1}^{2} \sum_{t=1}^{\mathcal{L}_{i}} \zeta_{i, t} \frac{\gamma^{t-1}}{\bar{\gamma}_{i}^{t} \Gamma[t]} \exp \left(-\frac{\gamma}{\bar{\gamma}_{i}}\right),
$$

where $\mathcal{L}_{1}=L-1, \mathcal{L}_{2}=1$ and

$$
\zeta_{i, t}=\left.\frac{1}{\left(\mathcal{L}_{i}-t\right) !\left(-\bar{\gamma}_{i}\right)^{\mathcal{L}_{i}-t}} \frac{\partial^{\mathcal{L}_{i}-t}}{\partial \omega^{\mathcal{L}_{i}-t}}\left(1-\bar{\gamma}_{i} \omega\right)^{\mathcal{L}_{i}} \varphi_{\gamma \mid \phi}(\omega)\right|_{\omega=\frac{1}{\bar{\gamma}_{i}}}
$$

Error probability analysis can be undertaken with the PDF of the output SINR conditioned on the $\phi_{1}$. For an illustration, coherent reception for equal-energy signals is considered, the SER conditioned on the output SINR is given by

$$
P_{\mathrm{mpsk}}\left(e \mid \gamma, \phi_{1}\right) \approx h \operatorname{erfc}(\sqrt{\gamma \lambda})
$$


The unconditional error performance can be obtained by averaging over the PDFs of $\gamma$ and $\phi_{1}$, where $\gamma$ is conditioned on $\phi_{1}$. Finally, this unconditional SER can be written as

$$
\begin{aligned}
P_{e} & =\int_{0}^{\infty} \int_{0}^{\infty} P_{\mathrm{mpsk}}\left(e \mid \gamma, \phi_{1}\right) p\left(\gamma \mid \phi_{1}\right) p\left(\phi_{1}\right) d \gamma d \phi_{1} \\
& =\int_{0}^{\infty} p\left(\phi_{1}\right) d \phi_{1} \int_{0}^{\infty} P_{\mathrm{mpsk}}\left(e \mid \gamma, \phi_{1}\right) p\left(\gamma \mid \phi_{1}\right) d \gamma
\end{aligned}
$$

where the order in which the integration is performed is interchanged in (6.17). With the PDF in (6.15), the integral $\int_{0}^{\infty} P_{\mathrm{mpsk}}\left(e \mid \gamma, \phi_{1}\right) p\left(\gamma \mid \phi_{1}\right) d \gamma$, which is the error performance conditioned on the eigen-value $\phi_{1}$, can be evaluated. This integral is denoted as $P\left(e \mid \phi_{1}\right)$. Thus, $P\left(e \mid \phi_{1}\right)$ can be expressed as

$$
P\left(e \mid \phi_{1}\right)=\sum_{i=1}^{2} \sum_{t=1}^{\mathcal{L}_{i}} \zeta_{i, t} \frac{h \Gamma[t+1 / 2]}{\sqrt{\pi} \Gamma[t]} B_{x_{i}}[t, 1 / 2],
$$

where $x_{i}=\frac{1}{\gamma_{i}+1}$ and $B_{x}[\cdot, \cdot]$ is the incomplete beta function.

The SER at the relay performing optimum combining for a desired user is given as

$$
P_{e} \simeq \int_{0}^{\infty} P\left(e \mid \phi_{1}\right) p\left(\phi_{1}\right) d \phi_{1}
$$

It is important to note that the integral in (6.19) can easily be evaluated using numerical means.

The next step is to formulate the E2E error performance. It will be assumed that each relay experiences independent error rates and that any error committed by the cooperating relay results in an error being committed at the destination. Therefore, the actual system performance could be better than the results that are estimated using these assumptions. The reason is that it is possible for an error to occur at the relays and these erroneous bits result in correct bit decisions at the destinations. The E2E error rate can be expressed as

$$
P_{e, \mathrm{E} 2 \mathrm{E}}=1-\prod_{i=1}^{2}\left(1-P_{e, i}\right)
$$

where in the two-hop scenario $P_{e, 1}$ is the error rate at the first hop (relay) and $P_{e, 2}$ is the error rate at the destination (the second hop). 


\subsection{Error Rate for Transmit Diversity}

The two transmit diversity described [10] offers an error rate that is exactly the same with receive diversity. This implies that with proper power adjustment, the performance of the transmit diversity schemes $(2 \times 1$ and $2 \times 2)$ can be evaluated using those of the corresponding receive diversity schemes which we have already derived. However, for the Alamouti $2 \times 1$ transmit diversity scheme, for binary PSK the error rate is given as in (2.7) and for M-ary PSK, the SER expression in (4.19) given as

$$
P_{e, 2}=\frac{h \Gamma[L m+1 / 2]}{\sqrt{\pi} \Gamma[L m]} B_{\mu}[L m, 1 / 2],
$$

can be used, where $\mu=\frac{m}{\lambda \bar{\gamma}+m}$ and $\bar{\gamma}$ is the adjusted SNR. Note that $L=2$ for $(2 \times 1)$ and $L=4$ for $(2 \times 2)$ transmit schemes and $m=1$ for Rayleigh fading.

\subsubsection{Numerical Examples: Performance of Relay with SDMA (Op- timum Combining)}

Figure 6.5 shows the BER curves for the desired user at the relay that nulls out a single interfering user. The BPSK modulation is used. This user comes with different average power. We have considered $L=1$ and $L=2$ at the relay. A number of important observations can be derived from these curves. With a single antenna, the system can do little to reduce the impact of the interfering user. It is interesting to observe that with equal power scenario, the single antenna relay suffers from an error floor. For a moderate interfering user power $\left(\mathrm{SNR}_{1}=0\right.$ and $\left.\mathrm{SNR}_{2}=10 \mathrm{~dB}\right)$, the performance obtained for $L=1$ is worse than that of a single user Rayleigh fading network (denoted with asterisk).

The situation is different for $L=2$ where it is observed that for moderate interfering power, a diversity order greater than one can be obtained. However, when $\mathrm{SNR}_{2} \rightarrow \infty$, (40 $\mathrm{dB}$ has been used to illustrate this). It appears that one degree of freedom is being sacrificed for sending a null to the interferer. The relay is then left with one degree of freedom to provide 


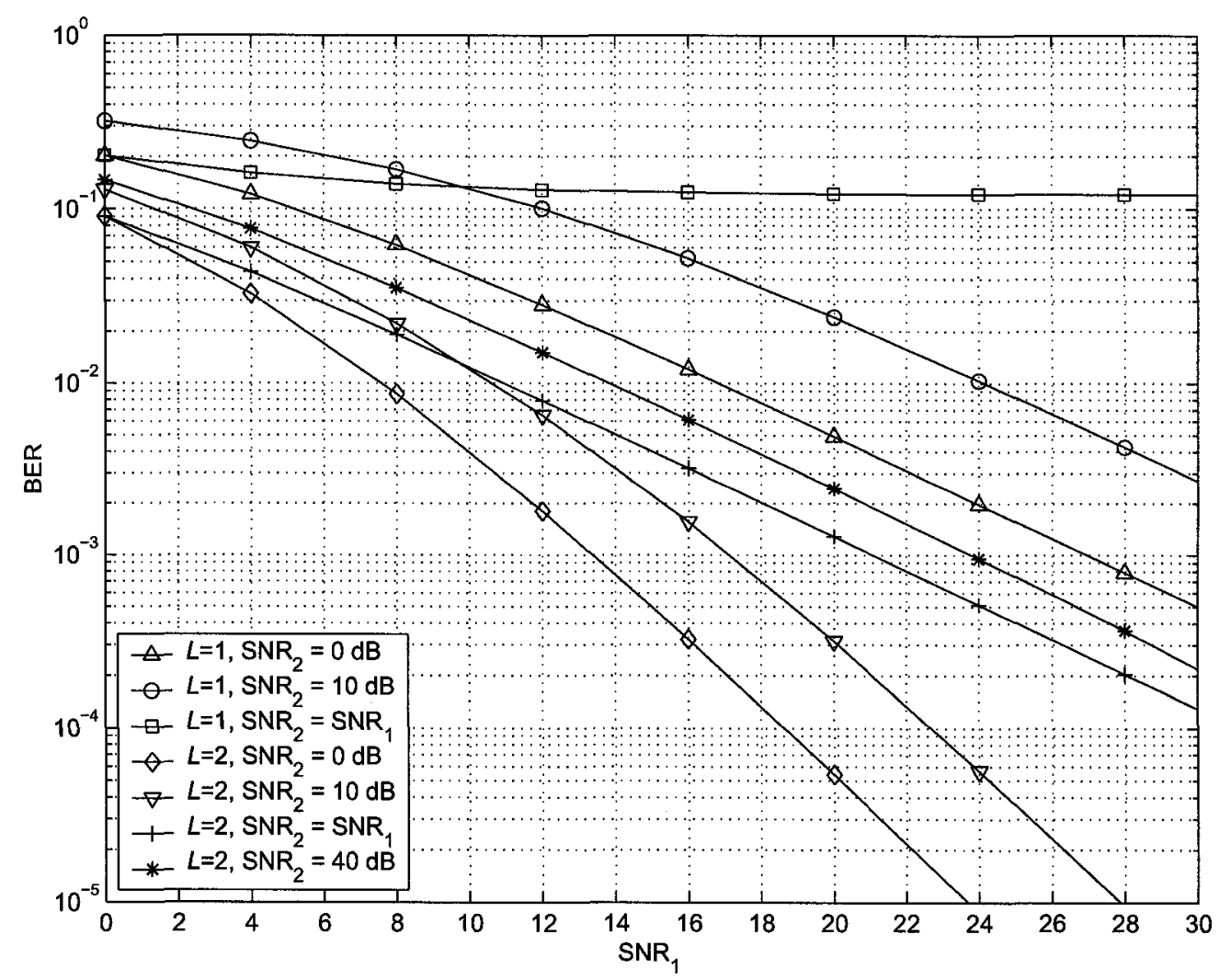

Figure 6.5: Performance of a relay performing optimum combining in Rayleigh fading channels. The BER of the desired user is shown for different levels of power of partner $(L=1, L=2)$. diversity gains. The slope of the curve (asterisks in Figure 6.5) indicates that diversity order of one is obtained for $L=2$ and when $\mathrm{SNR}_{2}=40 \mathrm{~dB}$. Moreover, this curve matches closely the performance of BPSK in Rayleigh fading channel where diversity is not employed.

Figure 6.6 shows BER for the desired user for $L=5$. It can be observed that as the number of antenna increases, the performance of the relay implementing optimum combining is not sensitive to the power of the partner. The SNR penalty incurred is within $1 \mathrm{~dB}$ when the partner's power increases from $0 \mathrm{~dB}$ to $40 \mathrm{~dB}$ for an error rate of $10^{-5}$. Moreover, for infinite power interferer the relay performance is bounded by the performance of $L-1$ diversity system with no interferer. As it can be derived from the slope of the curves given (Figure 6.6), 


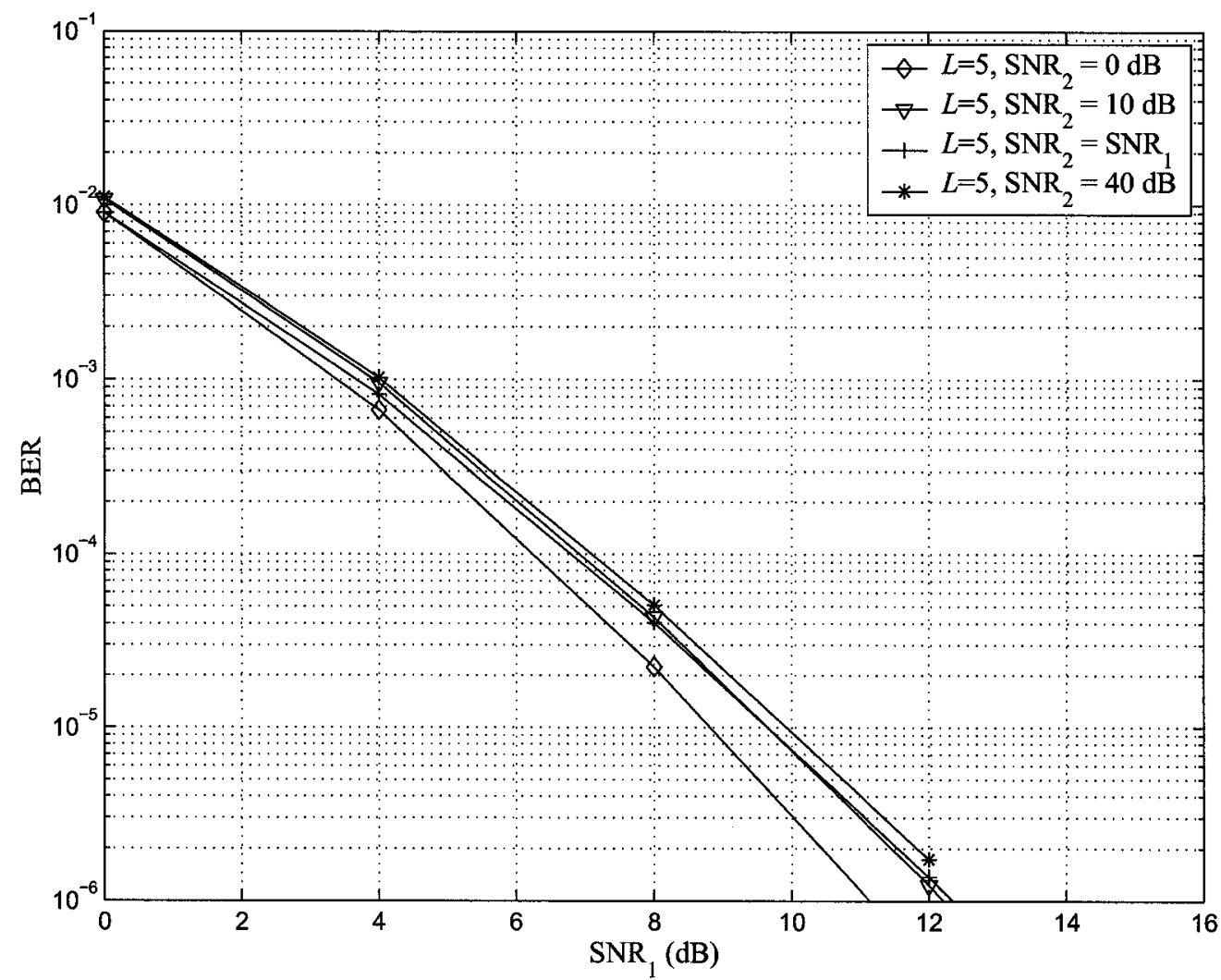

Figure 6.6: Performance of a relay performing optimum combining in Rayleigh fading channels. The BER of the desired user is shown for different levels of power of partner $(L=5)$.

a diversity of order four is obtained.

Figure 6.7 shows the symbol error rate performance at the relay for a system where 8-PSK modulation format is adopted for $L=2$ whereas Figure 6.8 shows that for the $L=3$ and $L=5$. The same trends as discussed previously are also observed. For the $L=3$ and $L=5$ we see that a diversity order of approximately 2.0 (for $L=3$ ) and 3.9 (for $L=5$ ) can be computed from the curves representing cases of $\mathrm{SNR}_{2}=40 \mathrm{~dB}$.

Having discussed the error performance at the relay, some examples of the E2E system performance are considered. First, simulation results are presented and discussed for an infinite power interferer. The analytical results take into account the scenarios where cooper- 


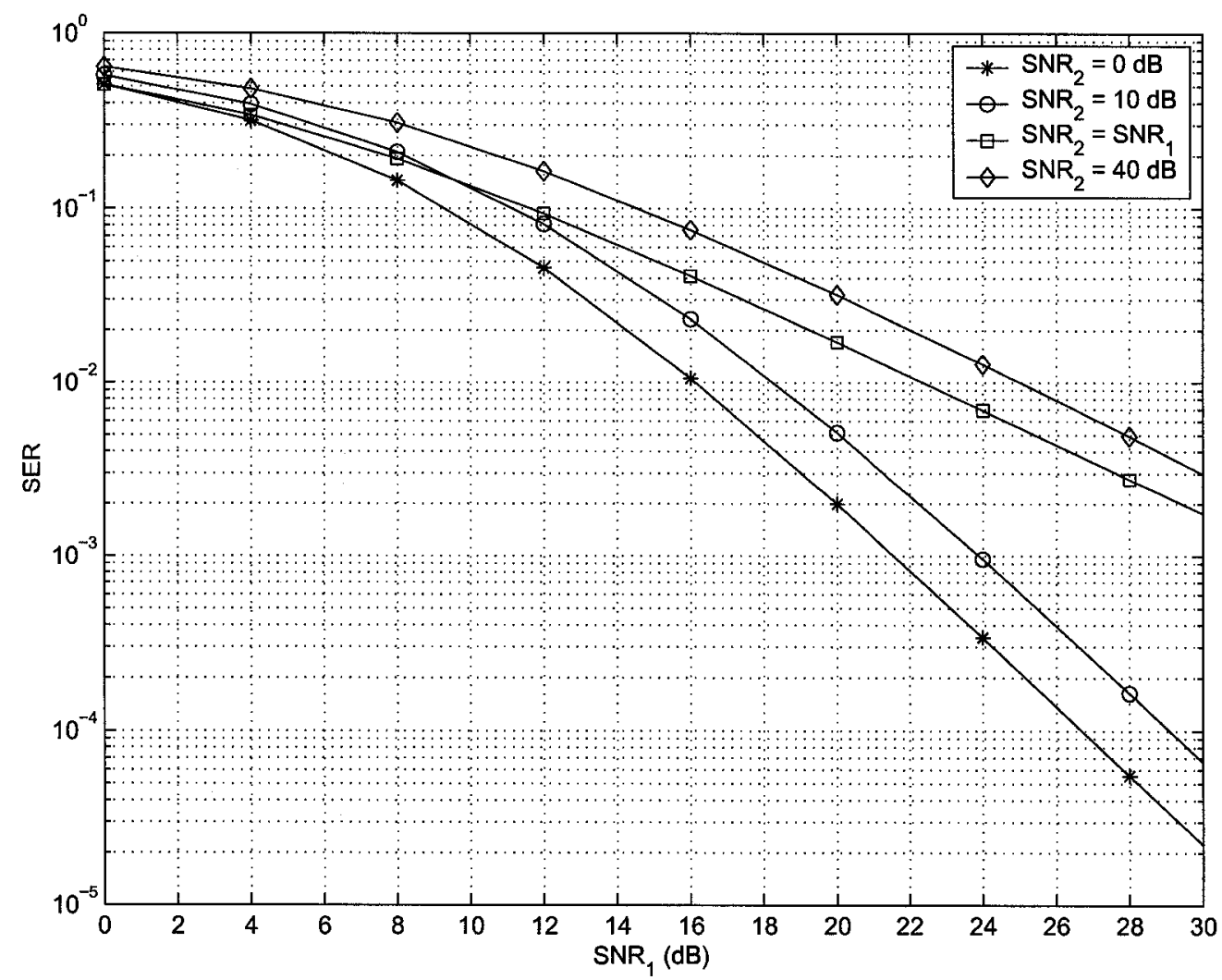

Figure 6.7: Performance of a relay performing optimum combining in Rayleigh fading channels for the desired user for different power levels of partner $L=2,8$-PSK.

ating partner has different values of power. Then, the simulated results with the cases where $\mathrm{SNR}_{2}=40 \mathrm{~dB}$ are compared.

\subsubsection{E2E Network Performance: Simulation Examples with Infi- nite Power Interferer}

Figure 6.9 shows some results of the relay-enabled user cooperation for the realization I. Each user has single antenna; the destination has one antenna but it can implement Alamouti-type receiver.

For $L=2$ the new scheme provides each of the two users in the network with an error rate 


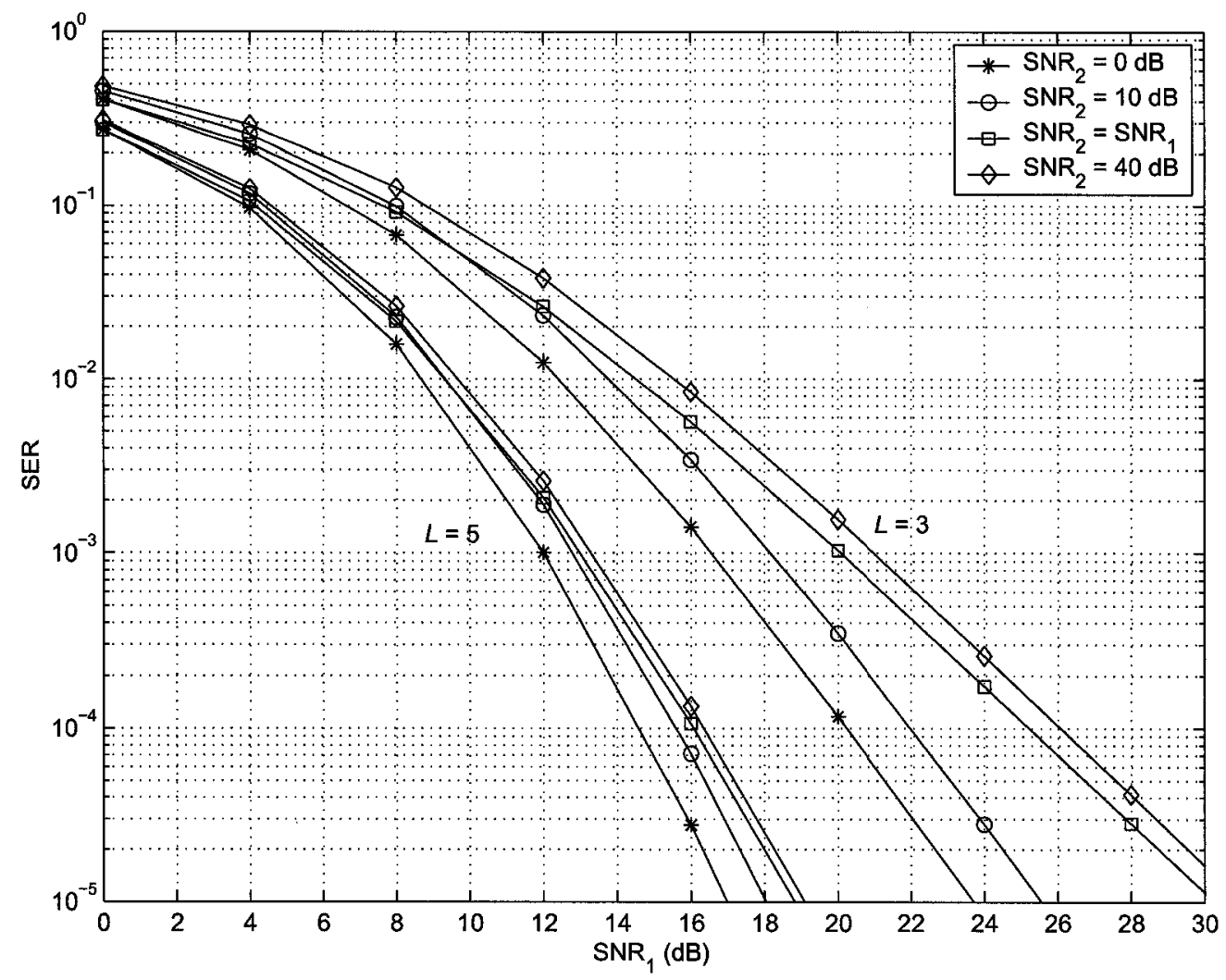

Figure 6.8: Performance of a relay performing optimum combining in Rayleigh fading channels for the desired user for different power levels of partner ( $L=3$ and $L=5$ ), 8-PSK. 


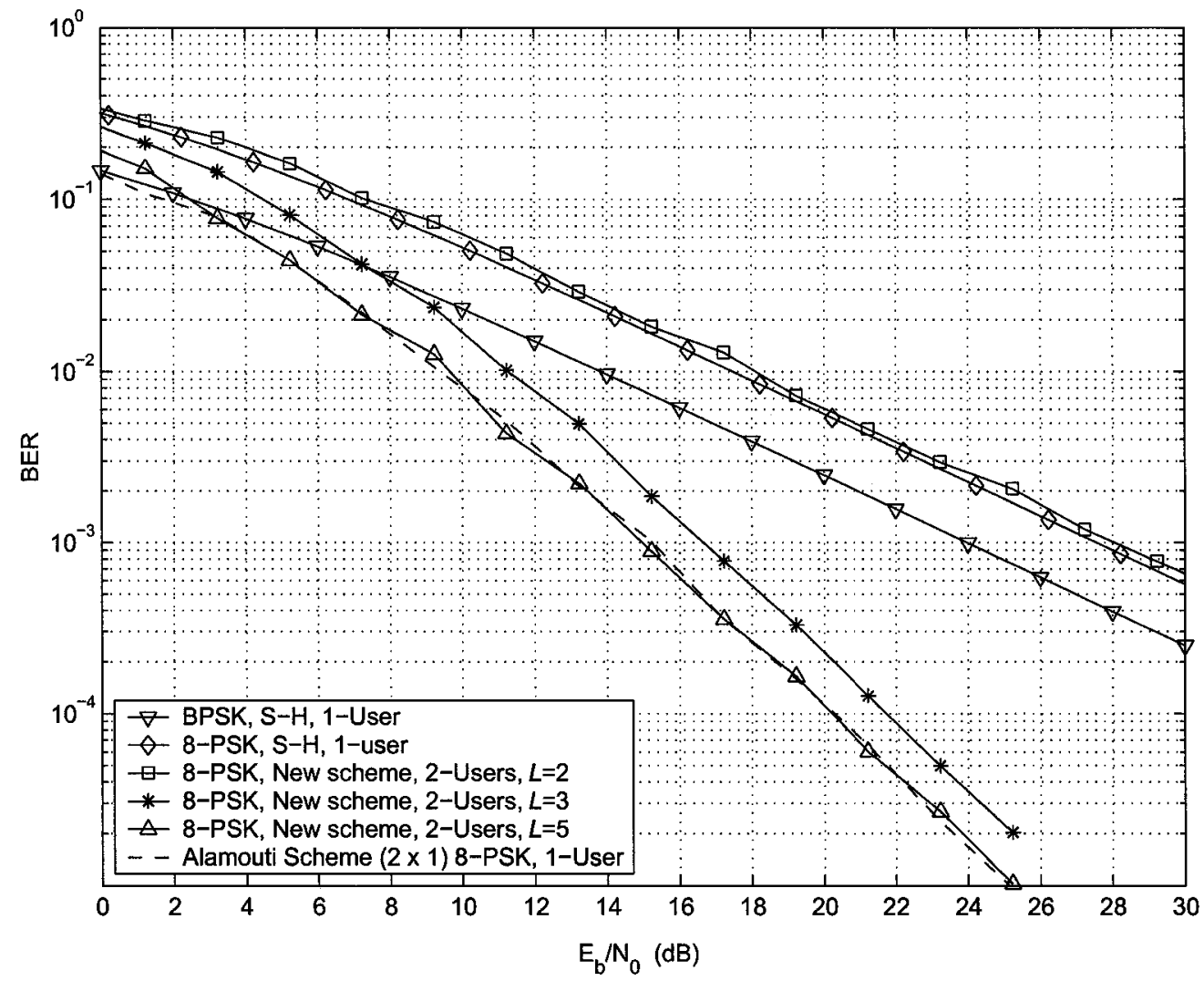

Figure 6.9: E2E performance of relay-enabled user cooperation in Rayleigh fading channels with one receive antenna at the destination (virtual $2 \times 1$ antennas in the second hop). 


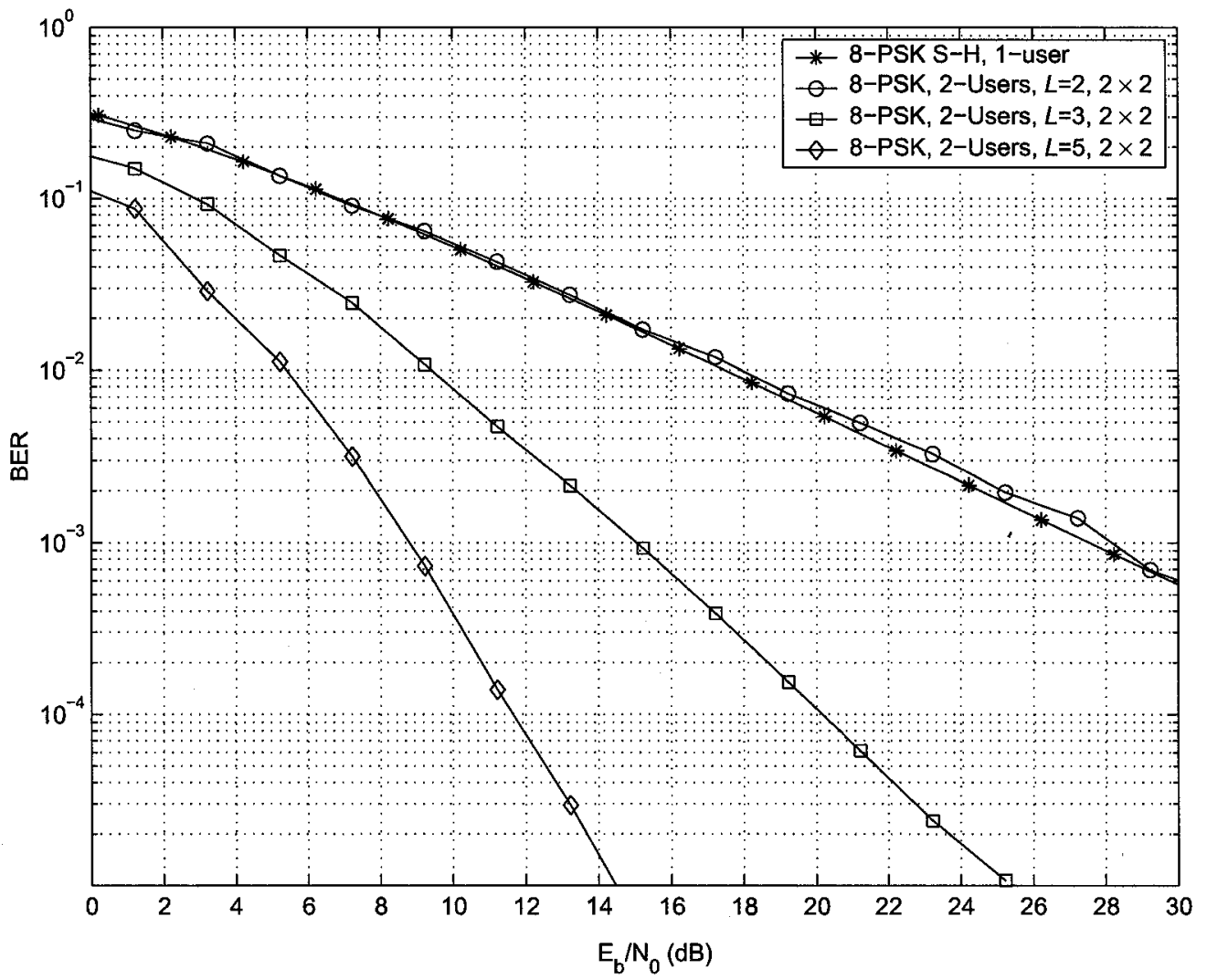

Figure 6.10: E2E performance of relay-enabled user cooperation in Rayleigh fading channels with two receive antennas at the destination (virtual $2 \times 2$ antennas in the second hop). 
that is not inferior than a single user network. In both networks, 8-PSK modulation format is adopted. This error performance advantage comes at the expense of $33 \%$ loss in spectral efficiency. The situation is different for large $L$. For instance, when $L=3$, the performance of each user in the new scheme is superior to that of the single user network using BPSK. This represents both diversity and spectral (multiplexing) gains. Considering the overall network, an improvement in spectral efficiency of $100 \%$ is achieved with the new scheme in addition to the large SNR gain at a low error performance. The new scheme achieves an SNR gain as large as $7.5 \mathrm{~dB}$ (for $L=3$ ) and $9 \mathrm{~dB}(L=5)$ over the BPSK at a BER of $10^{-3}$ (Figure 6.9). The margin of the gain is larger in low BER regime. Furthermore, the relay-enabled user cooperation scheme can provide each cooperating user with a performance that approaches that of a single user network implementing $2 \times 1$ Alamouti scheme. The scheme using SDMA requires only five antennas at the fixed relays to achieve this performance; however, it should be noted that each user terminal has one antenna. In the uplink, the base station is the destination. Hence, the number of antennas could certainly be more than one.

Figure 6.10 shows system performance when the destination uses two antennas. In this case, each antenna of the two (distributed) relays and the two antennas of the destination form a virtual $2 \times 2$ antenna scheme that could emulate conventional $2 \times 2$ Alamouti scheme (or MIMO channels). Comparing Figure 6.9 and Figure 6.10, a significant performance improvement due to the additional antenna at the destination is observed. For instance, at BER of $10^{-4}$, the virtual array $2 \times 2$ of the second hop is superior to its $2 \times 1$ counterparts by about $8 \mathrm{~dB}$.

\subsubsection{E2E Network Performance: Analytical Examples for Differ- ent Power Levels of Partner}

The E2E performance of the system is shown in Figure 6.11 for $L=2$ at the relay. Each of the two users have one antenna. The destination is equipped with a single antenna. The power 


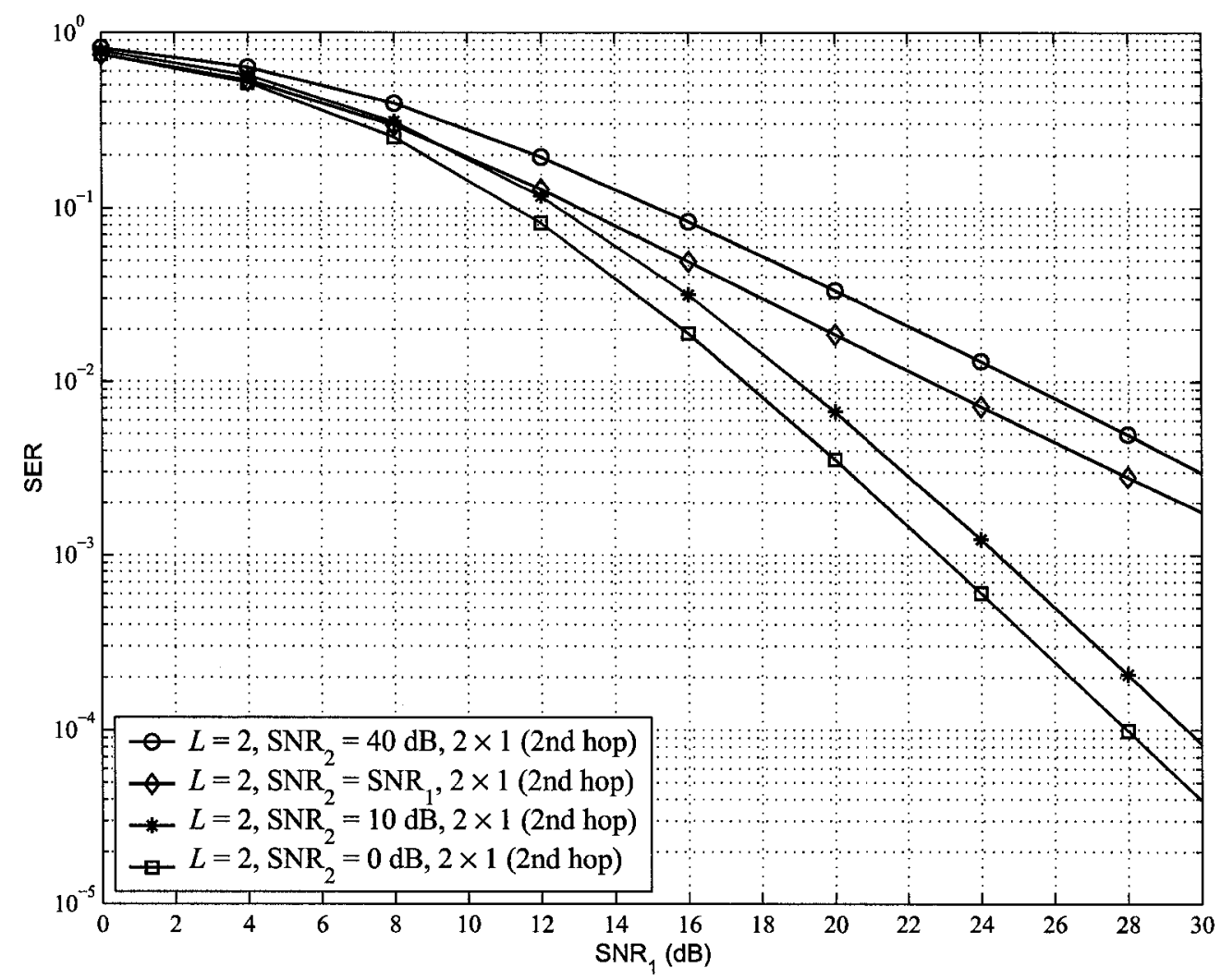

Figure 6.11: The E2E SER of the desired user for different power levels of partner $(L=2$, 8-PSK).

of the cooperating partner is set to $0,10,40 \mathrm{~dB}$ and $\mathrm{SNR}_{2}=\mathrm{SNR}_{1}$. It is observed that for $0 \mathrm{~dB}$, the E2E diversity order obtained for each partner is about 2 . However, for $\mathrm{SNR}_{2}=10$ $\mathrm{dB}$ this diversity is slightly reduced to 1.95 . When $\mathrm{SNR}_{2}$ is increased to $40 \mathrm{~dB}$, the overall diversity over rapidly dropped to 1 . It has been observed earlier that a minimum diversity of order two is required at the multi-antenna multi-relay cooperative scheme to provide an E2E diversity equal to the number of cooperating nodes. In the case of $\mathrm{SNR}_{2}=40 \mathrm{~dB}$, this criterion is not met at the relays resulting in drop in the $\mathrm{E} 2 \mathrm{E}$ gains.

Figure 6.12 shows the performance of the system for $L=3$. The power of the cooperating partner is set to $0,10,40 \mathrm{~dB}$, and $\mathrm{SNR}_{2}=\mathrm{SNR}_{1}$. A remarkable behaviour is observed for the 
curves which is different from that in Figure 6.11. Asymptotical comparison shows that all the curves yield the same diversity order. This diversity order is to the order of the number of cooperating relays, which is calculated (slopes of the curves) to be two. This is the same diversity order obtained for $L=2$ for moderate interference power. However, the antenna gain advantage is observed for $L=3$. It is important to note that the number of antenna at the relay has been used to improve the S-R channels only. This explains why the diversity order is restricted to the number of relay nodes. Had these antenna been engaged in transmit beamforming, it would have been possible to increase the diversity order. Another important point that can be deduced from these results is that in the multi-antenna multi-relay scheme, the minimum number of antenna required to achieve a diversity to the other of the multi-relay is two. This is the reason why for $L=3$, the diversity order obtained is the same as for all interfering power as well as for $L=2$ for moderate value of power of interferer.

A performance comparison for the simulated and analytical results is presented in Figure 6.13. The analytical results assume that the partner comes with a $40 \mathrm{~dB}$ power while the simulation results are for infinite power interferer. The curves demonstrate that analytical results, though it is approximate analysis, provide a reasonably close system performance.

\subsection{Chapter Conclusion}

There is growing interest in the infrastructure-based wireless relay/mesh networks in the academia, industry, and standardization bodies. Various proprietary mesh/relay networks solutions in unlicensed bands are being developed by industrial players. The emergence of the relay-enabled standards in the IEEE 802 family suggests that the interest and activity in relay-based communications system deployment will continue to grow. In other words, fixed relays will be an integral part of the future wireless systems.

As an add-on to network infrastructure-based fixed relays, this chapter investigates the cooperation of these relays to improve the end-to-end diversity gains and network multiplexing 


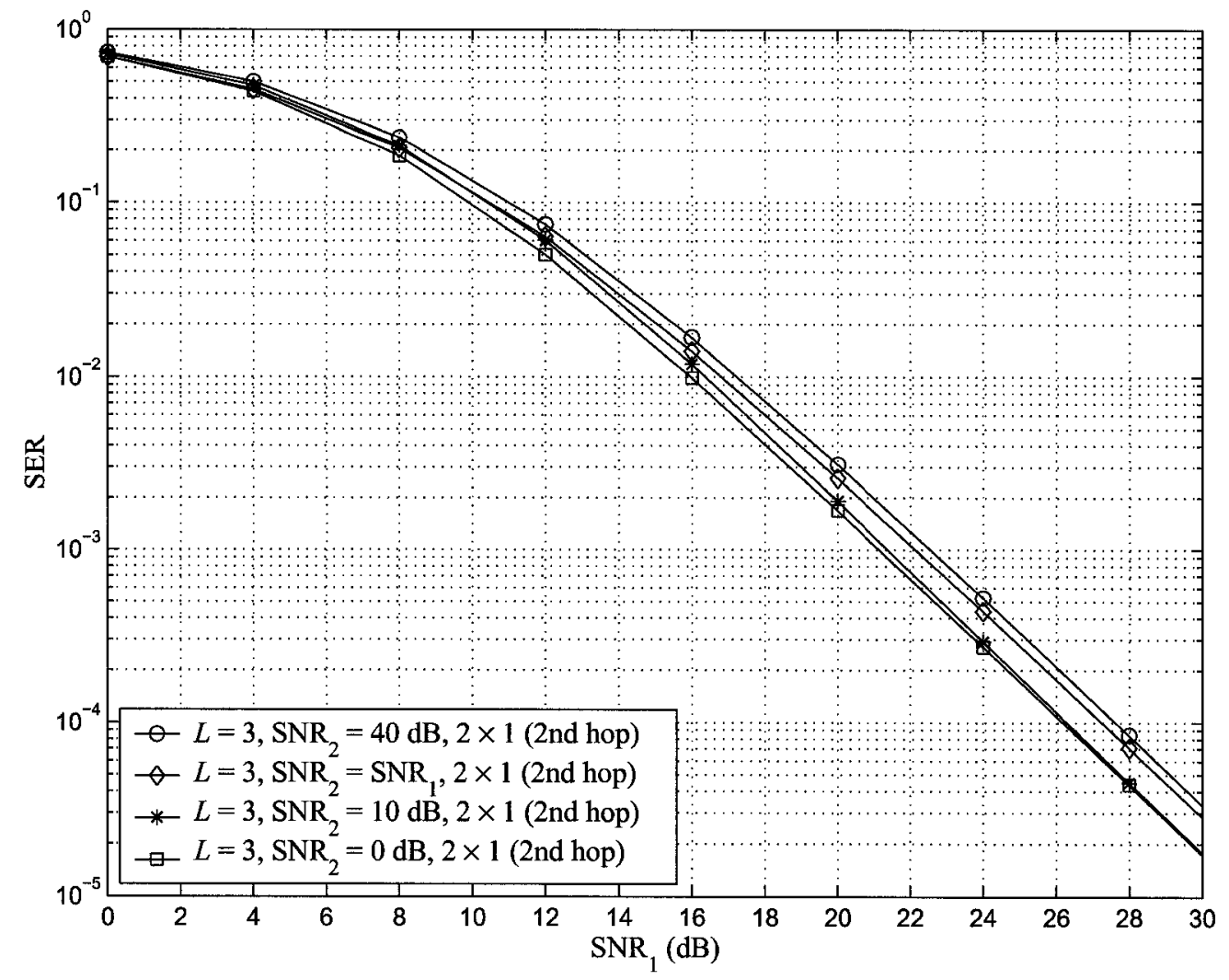

Figure 6.12: The E2E SER of the desired user for different power levels of partner $(L=3$, 8-PSK). 


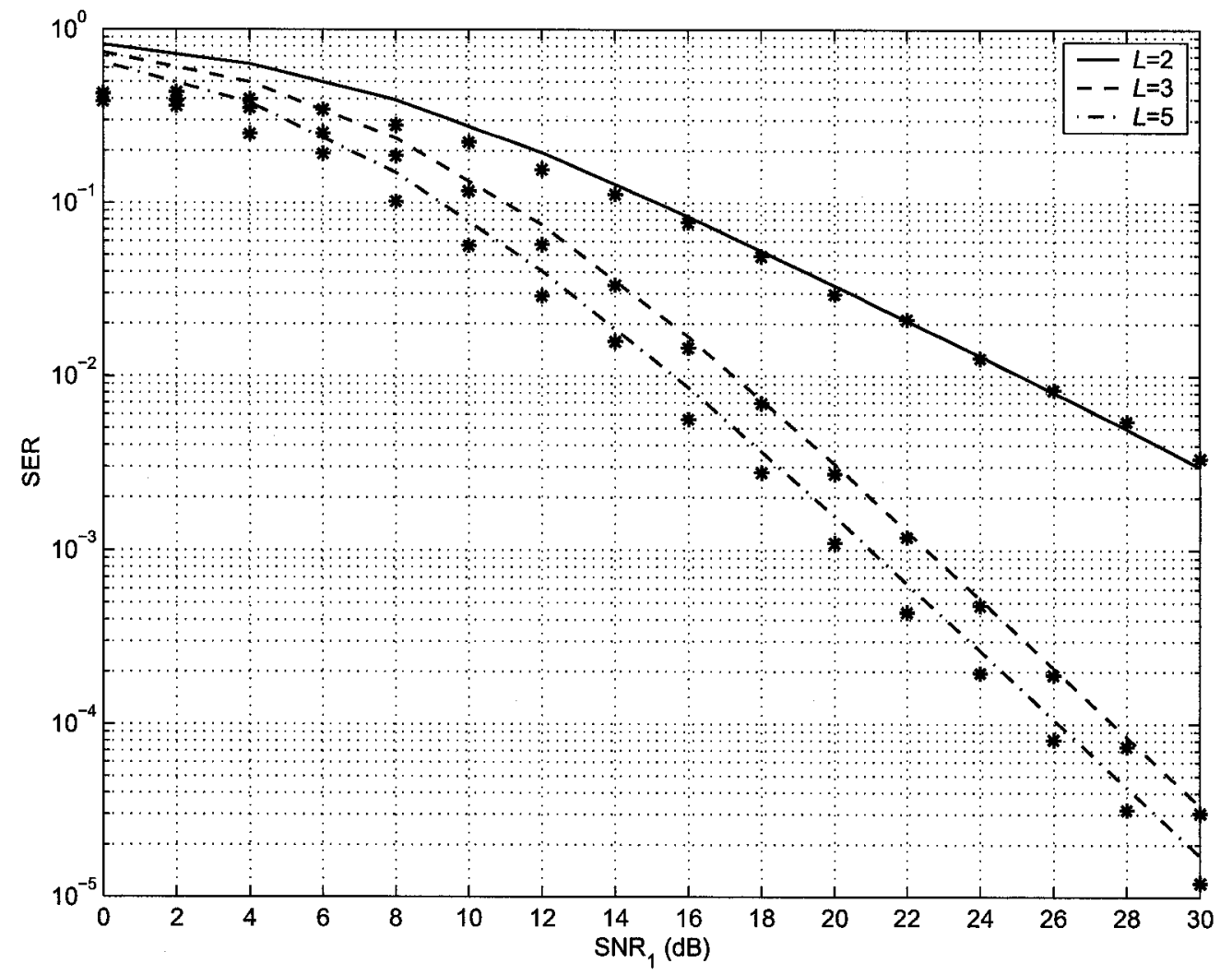

Figure 6.13: The E2E simulated SER of the desired user for infinite power of partner $\left(\mathrm{SNR}_{2}=\right.$ $\infty \mathrm{dB})$ and the analytical SER for $\mathrm{SNR}_{2}=40 \mathrm{~dB}$ (analysis) $(L=2,3,5,8$-PSK). 
gains. The proposed cooperation schemes are transparent to the users (i.e. users are ignorant of the cooperation), therefore, they present practical realizations for user cooperation as they naturally solve the issues of offering incentives for terminals to assist others as well as minimize the security and privacy concerns. In addition, obtained error performance and spectral efficiencies are superior to those of the referenced single-hop transmissions. Finally, the proposed schemes can be used in any network since current terminals do not require any modifications. Instead, the terminal complexity/cost are transferred to the relay level; it will be more economical to increase the cost of few relay stations rather than the cost of modifying thousands of terminals.

In this chapter, the analysis of the relay-enabled cooperative schemes is given in great detail for realization I. Realizations II and III, are discussed for the sake of implementation to avoid the problems associated with full duplex operation of relays. The analysis of these realizations is similar to that of realization I with few modifications. 


\section{Chapter 7}

\section{Conclusions and Recommendation}

The main focus of this thesis has been the development and application of advanced antenna and relaying techniques for wireless communication systems. The study addresses techniques that have practical implementation complexities and achieve high levels of performance. The motivation for this study comes from the current thrust in wireless communication technology. To deliver ubiquitous, reliable, high data rate wireless services requires significant radio resources such as scarce radio spectrum and link budget. It is therefore imperative to look for new, and cost-effective approaches and technologies that will improve among others, spectral efficiency and power utilization.

Multi-antenna techniques are well-known for improving the performance of wireless systems usually without an increase in bandwidth and excellent power savings. Space, cost, and signal processing constraints, among others, prevent the use of a large number of antennas at wireless terminals. Hence, the advances in point-to-point transmissions (antenna technologies and signal processing) alone appear handicapped in meeting the challenges of future wireless systems. Therefore, a fundamental change in the way systems are designed and deployed is required. The following approaches have been investigated in this thesis:

- The thesis investigates the use of infrastructure-based fixed relays for providing spatial diversity gains for a wireless terminal which, otherwise, has limitations in the number 
of antennas it can bear. In the contemporary context, relays are used for coverage extension or for distributing network capacity. As an add-on to network relays, the fixed relay nodes are engaged through two-hop cooperation with the source to provide E2E spatial diversity and network multiplexing benefits to these terminals. Thus, the burden of multiple antennas on wireless terminals is removed.

- Infrastructure-based fixed relays have the capability to carry multiple antennas. Therefore, the thesis investigates the multi-antenna aspects of cooperative two-hop networks. It also analyzes threshold maximal ratio combining and threshold selection combining of multi-relay multi-antenna schemes. A minimum of two antennas are required at the relay to yield an end-to-end diversity order of $N_{R}+1$ by the multi-antenna multi-relay cooperative scheme. $N_{R}$ is the number of relays. Any increase in the number of antennas only increases the antenna gains. Threshold decoding is employed as an additional measure towards combating error propagation. Furthermore, it was found that with a large number of antennas $(L)$ at the cooperative relays, threshold decoding is not necessary. However, for low $L$, there is an optimum choice for the threshold.

- The cooperative multi-antenna fixed relay scheme is extended to asymmetric channels. In symmetric networks, the multiple relays are either treated as being at the same location as the source or that all the relays are at the same location and midway between the source and the destination. This is the assumption in most of the previous works on the subject. Realistic scenarios are the ones where these relays face different channel strength to the destination. The thesis provides analytical framework for evaluating the performance of asymmetric relay networks. Therefore, it is easy to investigate different network topologies.

- Furthermore, the thesis also proposes relay-enabled user cooperation. This cooperation diversity scheme is proposed to avoid the problems related to the explicit user coop- 
eration diversity schemes. In this scheme, two users are engaged in the cooperation through two fixed relays without their knowledge. There are many benefits of this approach. First, the privacy of the users is not compromised since partners do not detect each other's data as it is the case with explicit user cooperation. Second, sanctions or rewards to facilitate user cooperation are not required. Third, network quality and services are not user-dependent. Fourth, this cooperation does not require any modifications on current terminals. The E2E system performance has been investigated through simulations and analytical efforts. The analysis of the relay-enabled cooperative schemes is given for the classification called realization I. Two other system implementation strategies are motivated to circumvent the problems of full duplex operation of relays. The analysis of these realizations is simple extension of that of realization I. Finally, the proposed scheme provides a bit error rate and network spectral efficiency that are superior to the referenced conventional transmissions.

- The thesis proposes a microdiversity-augmented macrodiversity architecture to combat two fading phenomena (small-scale fading and shadowing) in one shot. The proposed system works as follows. In the $K$-macro/ $L$-microdiversity antenna structure, macrodiversity selects the best among $K$ ports, using certain criteria, and of the $L$ signals available at that port $l_{\mathrm{o}}$ strongest are selected for diversity combining. The GSC is employed as a receiver processor for the microdiversity component since it represents a reasonable system complexity and cost compromise compared with a full blown MRC.

- With microcellular networks mind-set, a different approach to the conventional cellular concept by eliminating the need for expensive base station resources is proposed. Instead, a wireless network with cost-efficient radio access ports which are linked to a central unit (CU) is considered. The thesis:

- introduces novel means of implementing macro-selection (Scheme II) and demon- 
strates this scheme's performance superiority over the conventional method.

- explores macrodiversity MRC in microcellular systems referred to as Scheme III, which can be viewed as a natural extension of the CDMA cellular soft handover.

- provides the outage performance and error rate analyses for the architectures that are proposed.

- Finally, after illustrating the potential advantages of the proposed architectures and algorithms as compared to the referenced conventional schemes, the thesis then draws attention to some practical, complexity and implementation issues needed to be considered towards realizing these outlined benefits.

\subsection{Recommendation for Future Research}

- Quantifying the logical performance limits of the proposed hybrid macro/micro antenna architecture is another promising research avenue to be explored. For example, in the literature on classical diversity schemes, system performance criteria like diversity order and asymptotic behavior (a logical limit) are adequately quantified; hybrid micro/macrodiversity systems in the presence of composite fading on the other hand, do not easily lend themselves to these limits.

- The investigation of the performance of the proposed cooperative relaying schemes are based on the analysis of single cell model. Future research could extend it to multi-cell scenarios considering issues like intercell and/or co-channel interference.

- In the multi-antenna multi-relay cooperative schemes the E2E diversity gains does not require the decoding threshold for large number of antennas $(L)$ at the relays. However, for small $L$, the optimum threshold is chosen heuristically for the Rayleigh fading. In single antenna cooperative relaying this threshold is crucial. Thus, the threshold issue is 
an important one that needs to be treated in a comprehensive manner. Future research can focus on the generalization of the optimum threshold considering all the system parameters. As a matter of fact, a paper addressing the selection of the optimum threshold recently appeared in [59]. However, their set-up is much simpler than the systems discussed in this thesis. The authors consider single relay, single antenna at the relay and Rayleigh channel model.

- Synchronization issues related to the proposed fixed relay networks need to be investigated. Though synchronization in fixed relay networks is believed to be much less challenging and stringent than for terminal relaying, such studies may provide valuable insights on the issues discussed in Sec. 4.5 as well as provide quantitative information on control and signaling overhead required to initiate and sustain the cooperation.

- Network latency is an important part of network performance for many applications. Therefore, the latency imposed on the network by the cooperative schemes needs to be investigated. By doing this the impact on a wide range of applications, especially those with stringent delay requirements, will be understood. Such studies would provide valuable information on efficient integration of the cooperative schemes into wireless systems. 


\section{Appendix A}

\section{Published and Submitted Work}

- Journal, Published and in review

- Abdulkareem Adinoyi and Halim Yanikomeroglu, "Practical capacity calculation for time-hopping ultra-wide band multiple-access communications", IEEE Communications Letters, vol. 9, no. 7, pp. 601-603, July 2005 [Appendix B].

- Abdulkareem Adinoyi and Halim Yanikomeroglu, "Hybrid macro/microdiversity techniques in the reverse-link wireless communication networks", submitted to IEEE Transaction on Wireless Communications, November 2004, revised November 2005 [Extracted from Chapter 3].

- Abdulkareem Adinoyi and Halim Yanikomeroglu, "Cooperative relaying in multiantenna relay networks", accepted to IEEE Transactions on Wireless Communication, May 2006 [Extracted from Chapter 4].

- Journal, in preparation

- Abdulkareem Adinoyi and Halim Yanikomeroglu, "Relay-enabled user cooperative diversity schemes: system descriptions and performance analysis," in preparation. 
- Abdulkareem Adinoyi and Halim Yanikomeroglu, "Error probability analysis of cooperative fixed wireless relays with arbitrary relay locations," in preparation.

- Abdulkareem Adinoyi, Keivan Navaie, Furuzan Atay, Mahmoud Rahman and Halim Yanikomeroglu, "Multi-hop wireless cellular networks: possibilities, technologies, and challenges," in preparation for IEEE Wireless Communications.

- D. Schultz, R. Pabst, B. Walke, K. Navaie, A. Adinoyi, H. Yanikomeroglu, N. Johansson, K. Sunell, and W. Zirwas, "Multi-hop protocols for relay based deployment concepts," in preparation for IEEE Network Magazine Special Issue on Evolution toward $4 G$ Wireless Networking.

\section{- Conferences}

- Abdulkareem Adinoyi and Halim Yanikomeroglu, "On the performance of cooperative wireless fixed relays in asymmetric channels," submitted to IEEE GLOBECOM, November 2006.

- Abdulkareem Adinoyi and Halim Yanikomeroglu, "Spectral efficiency and diversity gains through cooperative wireless fixed relays," submitted to IEEE Vehicular Technology Conference, September 2006.

- Abdulkareem Adinoyi and Halim Yanikomeroglu, "Multi-antenna aspects of parallel fixed wireless relays," IEEE Wireless Communications and Networking Conference, April 2006.

- Yijia Fan, Abdulkareem Adinoyi, John Thompson, and Halim Yanikomeroglu, "Space diversity for multi-antenna multi-relay channels," 12th European Wireless Conference, April 2006.

- Abdulkareem Adinoyi and Halim Yanikomeroglu, "Practical communication through cooperative fixed relays," Wireless World Research Forum (WWRF\#15), December 2005 . 
- Abdulkareem Adinoyi and Halim Yanikomeroglu, "On the performance macro/microdiversity in the reverse-link wireless networks," IEEE Wireless Communications and Networking Conference, vol. 2, pp. 973-977, March 2005.

- Abdulkareem Adinoyi, Halim Yanikomeroglu, and Sergey Loyka, "Hybrid macroand generalized selection combining microdiversity in lognormal shadowed Rayleigh fading channels," IEEE International Conference on Communications, vol. 1, pp. 244-248, June 2004. 


\title{
Appendix B
}

\section{Practical Capacity Calculation for}

\author{
Time-Hopping Ultra-wide Band
}

Multiple-Access Communications

In this letter the practical capacity, known as the cutoff rate, of time-hopping (TH) ultra-wide band $(U W B)$ communication system is evaluated for multiple-access channels. The cutoff rate can be used for determining various system trade-offs. For instance, it is shown in this letter that if synchronization problems would preclude high spreading factors, a suitable number of hops can be used instead to achieve the same performance. Moreover, it is demonstrated that the cutoff rate evaluated here can be a fast way of gaining insights into the multiuser capacity of TH-PPM UWB systems.

\section{B.1 Introduction}

Considerable interest has developed for time-hopping (TH) ultra-wide band (UWB) multipleaccess communication systems [93]. This is due to UWB's appealing features; for instance, it does not require a sinusoidal carrier, it can highly resolve multipath, and it enjoys low 
probability of detection and interception. These features make UWB technology a promising option for high data rate communications.

The error exponent and cutoff rate are practical and important information-theoretic measures used extensively in the literature for comparing coding scheme performance [94] and constellation design [95]. These parameters set the bound on the performance and determine both the achievable rate and magnitude of the random-coding error exponent with practical modulation/coding schemes. This letter evaluates the cutoff rate for TH-PPM UWB multiple-access channels.

\section{B.2 Random Coding Error Exponent}

The ensemble average probability of block decoding error using a maximum likelihood decoding is bounded by

$$
P_{e} \leq \exp \left(-N\left[E_{0}(P(x), \rho)-\rho \mathcal{R}\right]\right)
$$

where $N$ is the code block length, and $\mathcal{R}$ is the information rate per channel symbol forming the ensemble of $(N, \mathcal{R})$ block codes in which each alphabet is selected with probability $P(x)$. The argument $E(\mathcal{R}, \rho)=\left[E_{0}(P(x), \rho)-\rho \mathcal{R}\right]$ is known as the channel random coding error exponent [94] where $\rho$ and $P(x)$ are chosen such that maximum exponent value is obtained, because (B.1) indicates that for some given codes with the same complexity (measured through $N$ ) and same rate $(\mathcal{R})$, the channel having the largest $E(\mathcal{R}, \rho)$ value will result in the lowest error probability.

In this paper $\rho=1$ is of interest and is referred to as the cutoff rate, $\mathcal{R}_{0}\left(=E_{0}(P(x), 1)\right)$, which can be expressed as [95]

$$
\mathcal{R}_{0}=\max _{P_{j}}\left\{-\ln \left[\sum_{j} \sum_{k} P_{j} P_{k} \int_{\mathbf{y}} \sqrt{p(\mathbf{y} \mid j) p(\mathbf{y} \mid k)} d \mathbf{y}\right]\right\},
$$

where $P_{j}$ is the a priori input probability, $p(\mathbf{y} \mid j)$ is probability density function of the output vector given that $j$-th signal was transmitted. If $\log _{2}$ is used instead of $\ln$ in (B.2), 
$\mathcal{R}_{0}$ is in bits/transmitted waveform. Cutoff rate has been considered the practical capacity beyond which communication would be very expensive. Even though the recent experience with the near-capacity performance of turbo codes (TC) appears to threaten this belief, a substantial amount of price in terms of complexity and delay is still paid through long interleaver and iterative decoding of TC. In sequential decoding applications, cutoff rate remains a valuable parameter which provides insight complementary to that acquired by the study of capacity [96].

Let us consider a time hopping $\mathcal{N}_{u}$-user UWB system employing M-ary PPM. A typical $k^{\text {th }}$ user's received signal with perfect power control takes the form [93, 97]

$$
s^{(k)}(t)=\sqrt{\frac{E_{s}}{N_{s}}} \sum_{j=0}^{N_{s}-1} p_{r x}\left(t-j \mathcal{T}_{f}-c_{j}^{(k)} \mathcal{T}_{c}-\mathcal{D}_{j}^{(k)}\right),
$$

then the total multiple-access received signal can be represented as

$$
y(t)=s^{(1)}\left(t-\tau^{(1)}\right)+\sum_{k=2}^{\mathcal{N}_{u}} s^{(k)}\left(t-\tau^{(k)}\right)+n(t),
$$

where user 1 is the user of interest. In (B.3) and (B.4), $p_{r x}(t)$ is the basic pulse with a duration of $\mathcal{T}_{p}, \mathcal{T}_{f}$ is the frame time, $\mathcal{T}_{s}=N_{s} \mathcal{T}_{f}$ is the symbol duration, $c_{j}^{(k)}$ is the hopping code, $\mathcal{D}_{j}^{(k)} \epsilon\left\{\delta_{1}, \cdots, \delta_{M}\right\}$ represents the PPM time shift corresponding to the modulating data sequence of user $k$ at hop $j, \tau^{(k)}$ represents time asynchronism, $E_{s}$ is pulse (symbol) energy, and $n(t)$ is the additive white Gaussian noise (AWGN) with two-sided power spectral density $N_{0} / 2$.

We assume that the receiver is in perfect synchronism with the user of interest so that the correlation receiver can be implemented. With this assumption, the receiver for the M-ary PPM scheme is composed of $M$ filters matched to the template functions $\psi_{i}^{(1)}$, defined as

$$
\psi_{i}^{(1)}(t)=p_{r x}\left(t-\delta_{i}-\tau^{(1)}\right), \quad i=1,2, \cdots, M .
$$

Each matched filter computes as output the following decision statistics

$$
y_{i}=\sqrt{\frac{N_{s}}{E_{s}}} \sum_{j=0}^{N_{s}-1} \int_{j \mathcal{T}_{f}}^{(j+1) \mathcal{T}_{f}} y(t) \psi_{i}^{(1)}\left(t-j \mathcal{T}_{f}-c_{j}^{(1)} \mathcal{T}_{c}-\tau^{(1)}\right) d t .
$$


We can write $y_{i}=D_{i}+I_{i}+N_{i}$ where $D_{i}$ contains the signal to be detected, $I_{i}$ is the multipleaccess interference (MAI) and $N_{i}$ is the noise. ${ }^{1}$ The variance of $N_{i}, \sigma_{N_{i}}^{2}$, is obtained as

$$
\sigma_{N_{i}}^{2}=\frac{N_{s}}{E_{s}} \sum_{j=0}^{N_{s}-1} \sum_{k=0}^{N_{s}-1} \delta_{k j} \sigma_{n}^{2} \mathrm{R}(0)=N_{s}^{2} \mathrm{R}(0) \sigma_{n}^{2} / E_{s},
$$

where $\sigma_{n}^{2}=N_{0} / 2$ and $\mathrm{R}(\Delta)$ is the correlation of the basic pulse for a lag $\Delta$. Similarly, $D_{i}=N_{s} \mathrm{R}(0)$, and the MAI part is expressed as

$$
I_{i}=\sum_{k=2}^{\mathcal{N}_{u}} \sum_{j=0}^{N_{s}-1} \mathrm{R}\left(\Delta_{j}^{(k)}\right)
$$

where $\Delta_{j}^{(k)}=\left(c_{j}^{(1)}-c_{j}^{(k)}\right) \mathcal{T}_{c}+\left(\mathcal{D}_{j}^{(k)}-\mathcal{D}_{j}^{(1)}\right)+\left(\tau^{(k)}-\tau^{(1)}\right)$ is a random time lag between users 1 and $k$ in the $j$-th hop frame. We assume that all the time hopping $\left(c_{j}\right.$ 's) are random, and hence, the monocycle time shift $c_{j}^{(k)} \mathcal{T}_{c}$, and the time delays are i.i.d with uniform distribution over a frame interval. Since the UWB pulse duration $\mathcal{T}_{p}<<\mathcal{T}_{f}$, each interfering pulse contributes to only a single correlation operation (i.e., MAI pulses fall within the same UWB frame). Therefore, $\Delta_{j}^{(k)}$ is uniformly distributed in the interval $\left[-\mathcal{T}_{f}, \mathcal{T}_{f}\right]$ [98]. For large values of $\mathcal{N}_{u} \times N_{s}$, the probability density function (PDF) of $I_{i}$ converges to a Gaussian distribution. Without loss of generality, we consider rectangular monocycle pulse, although, Gaussian and Rayleigh pulses fit easily in the analysis as well. Defining a spreading factor $\beta=\mathcal{T}_{f} / \mathcal{T}_{p}$, the variance of $I_{i}$ can be expressed, with the help of [98], as

$$
\sigma_{I_{i}}^{2}=\sum_{k=2}^{\mathcal{N}_{u}} \sum_{j=0}^{N_{s}-1} \operatorname{var}\left[\mathrm{R}\left(\Delta_{j}^{(k)}\right)\right]=\left(\mathcal{N}_{u}-1\right) N_{s}\left(\frac{1}{3 \beta}-\frac{1}{4 \beta^{2}}\right) .
$$

It can be shown, by exploiting the orthogonality in the PPM M-ary signal set, that the

\footnotetext{
${ }^{1}$ The decision statistic for user $1, y_{i}^{(1)}$, is written as $r_{i}$ for notational convenience. The same simplified notation is used in the rest of the letter for other parameters related to user 1 as well, such as $D_{i}, N_{i}$ and $I_{i}$.
} 
distribution of the received signal $\mathbf{y}$ is given as

$$
\begin{aligned}
p\left(\mathbf{y} \mid \mathbf{x}_{j}\right) & =\left(\frac{1}{2 \pi \sigma_{\text {tot }}^{2}}\right)^{M / 2} \exp \left[-\frac{\left(y_{j}-\varpi_{p}\right)^{2}}{2 \sigma_{\text {tot }}^{2}}\right] \\
& \times \prod_{\substack{k=0 \\
k \neq j}}^{M-1} \exp \left[-\frac{\left(y_{k}-\varpi_{a}\right)^{2}}{2 \sigma_{\text {tot }}^{2}}\right] \\
& =p(y \mid j) \prod_{\substack{k=0 \\
k \neq j}}^{M-1} p(y \mid k)
\end{aligned}
$$

where, $\mathbf{x}_{j}=\left[x_{0}, \cdots, x_{j}, \cdots, x_{M-1}\right]$, is the transmitted signal and $\mathbf{y}=\left[y_{0}, \cdots, y_{j}, \cdots, y_{M-1}\right]$. In the above, $p(y \mid j)$ and $p(y \mid k)$ indicate the PDFs of the matched filter output when the desired signal is present and absent, respectively. The second PDF represents noise-only output. The parameter $\sigma_{t o t}^{2}$ denotes the total noise variance, $\varpi_{p}$ and $\varpi_{a}$ represent slot signal strengths when signal is present and absent, respectively. Using (B.10) with (B.2) and $P_{j}=1 / M$, $0 \leq j \leq M-1$, the uniform input distribution that maximizes $\mathcal{R}_{0}$ [99], the cut-off rate $\mathcal{R}_{0}$ can be expressed as

$$
\begin{aligned}
& \mathcal{R}_{0}=\max _{P_{j}}\left\{\begin{array}{l}
-\log _{2}\left[\sum_{l=0}^{M-1} P_{l}^{2}\left(\int_{-\infty}^{\infty} \sqrt{p(y \mid l) p(y \mid l)} d y\right)^{2}\right. \\
\left.+\sum_{k=0}^{M-1} \sum_{\substack{j=0 \\
j \neq k}}^{M-1} P_{j} P_{k}\left(\int_{-\infty}^{\infty} \sqrt{p(y \mid j) p(y \mid k)} d y\right)^{2}\right]
\end{array}\right\} \\
& =\log _{2}(M)-\log _{2}\left[1+(M-1) \exp \left(-\frac{\left[\varpi_{p}-\varpi_{a}\right]^{2}}{4 \sigma_{\text {tot }}^{2}}\right)\right],
\end{aligned}
$$

where $\varpi_{p}=E\left[y_{i} \mid\right.$ given that the desired signal is present $]=N_{s} R(0)$,

$\varpi_{a}=E\left[y_{i} \mid\right.$ given that the desired signal is absent $]=E\left[I_{i}+N_{i}\right]=0$, and $\sigma_{\text {tot }}^{2}=\sigma_{N_{i}}^{2}+\sigma_{I_{i}}^{2} . E[\cdot]$ denotes the mean operator.

Finally, we observe that when the network becomes heavily loaded, the cutoff rate asymptotically approaches zero, but the aggregate rate $\mathcal{R}_{0, a g g}\left(=\mathcal{N}_{u} \mathcal{R}_{0}\right)$ converges to a nonzero 


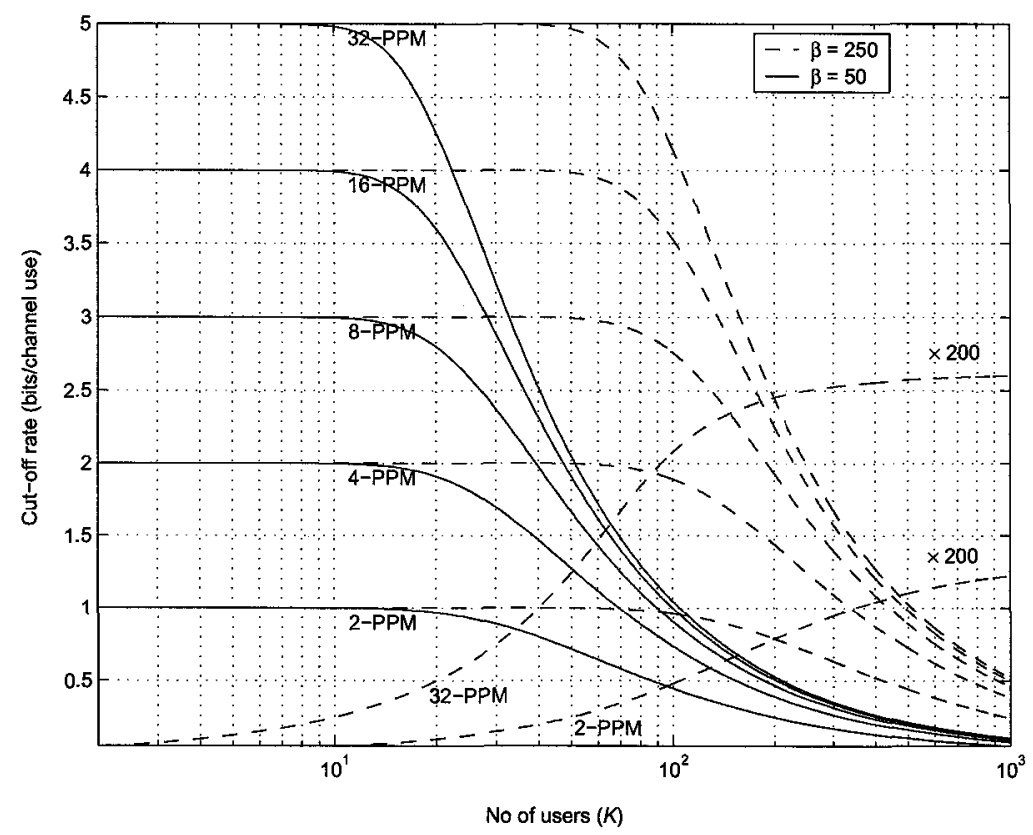

Figure B.1: Cutoff rates of M-ary PPM multiuser UWB for two different spreading factors at $\mathrm{SNR}=20 \mathrm{~dB}$ in the absence of time hopping $\left(N_{s}=1\right)$.

constant; this is confirmed by the asymptotic aggregate cutoff rate expression derived as

$$
\begin{aligned}
\mathcal{R}_{0, a g g} & =\lim _{\mathcal{N}_{u} \rightarrow \infty} \mathcal{N}_{u} \log _{2}\left[\frac{M}{1+(M-1) \exp \left(-\frac{\left[\varpi_{p}-\varpi_{a}\right]^{2}}{4 \sigma_{\text {tot }}^{2}}\right)}\right] \\
& \approx \frac{3 \beta(M-1)\left(\varpi_{p}-\varpi_{a}\right)^{2}}{2 N_{s} M \log _{e}(2)} .
\end{aligned}
$$

\section{B.3 Numerical Results}

Figure B.1 shows the cutoff rate of the UWB system versus number of users and also shown is the asymptotic behaviour of the aggregate cutoff rate for 2- and 32-PPM. We observe that a certain maximum number of users can be accommodated in order to achieve the maximum $\mathcal{R}_{0}$ $\left(=\log _{2}(M)\right)$. It is further observed that using a higher spreading factor delays the fast drop in the cutoff rate with respect to the number of users. Figure B.2 shows the cutoff rate as a 


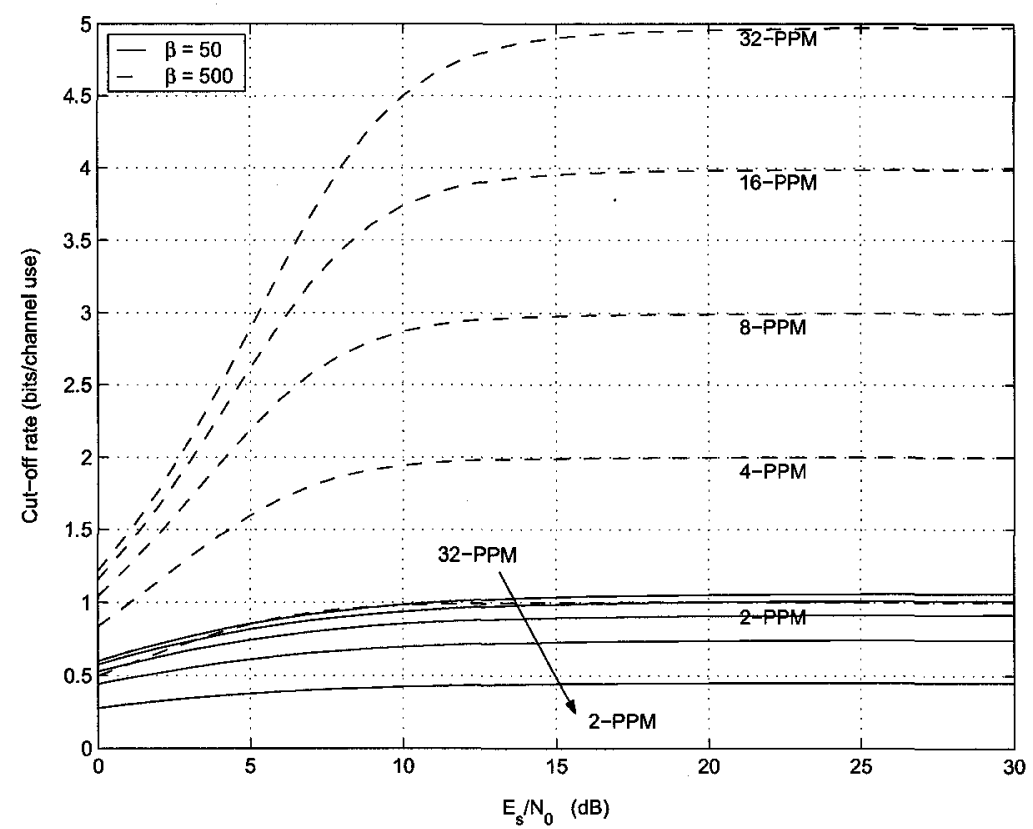

Figure B.2: Cutoff rates of M-ary PPM multiuser UWB for two different spreading factors for $\mathcal{N}_{u}=100$ in the absence of time hopping $\left(N_{s}=1\right)$. 
Table B.1: Code lengths for PPM signaling at rate $2.0 \mathrm{bits} / \mathrm{symbol}$

\begin{tabular}{||c|c|c|c|c|c|c||}
\hline \hline & \multicolumn{2}{|c|}{$8-\mathrm{PPM}$} & \multicolumn{2}{c|}{$16-\mathrm{PPM}$} & \multicolumn{2}{c|}{$32-\mathrm{PPM}$} \\
\hline Number of users & $P_{e} \leq 10^{-3}$ & $P_{e} \leq 10^{-6}$ & $P_{e} \leq 10^{-3}$ & $P_{e} \leq 10^{-6}$ & $P_{e} \leq 10^{-3}$ & $P_{e} \leq 10^{-6}$ \\
\hline 5 & 11 & 21 & 6 & 11 & 4 & 7 \\
\hline 15 & 14 & 28 & 7 & 14 & 5 & 10 \\
\hline 20 & 20 & 39 & 10 & 19 & 7 & 14 \\
\hline 30 & 107 & 214 & 21 & 42 & 14 & 28 \\
\hline \hline
\end{tabular}

function of SNR for a fixed number of users of $\mathcal{N}_{u}=100$. We observe that a heavily loaded channel results in an extremely low $\mathcal{R}_{0}$ when the spreading factor is relatively low $(\beta=50)$.

For example, 32-PPM could only operate at $1 \mathrm{bit} /$ symbol even at large SNR when $\beta=50$. Increasing the spreading factor yields a better performance (higher $\mathcal{R}_{0}$ ), but this is not always desirable because of the potential synchronization problems. The impact of time hopping $\left(N_{s}=4, N_{s}=8\right.$, and $N_{s}=10$ ) on $\mathcal{R}_{0}$ is shown in Figure B.3 for 2-PPM. It is observed that the capacity of $\mathrm{TH}$ system $\left(N_{s}=4\right)$ is approximately twice that of a non-hopping system $\left(N_{s}=1\right)$ for $\beta=50$.

\section{B.3.1 Coding Complexity Measure}

Consider that a waveform with a rate of 2 bits/symbol is desired. A natural choice would have been 4-PPM, but Figure B.2 indicates that 4-PPM and 8-PPM require about $12 \mathrm{~dB}$ and $4 \mathrm{~dB}$, respectively, for $\beta=500$. Therefore, 8-PPM has a power saving advantage if a suitable coding scheme with certain complexity, in Shannon sense, can be found for a given error rate. Table B.1 shows the computed block lengths (complexity) required to satisfy a specific probability of error for schemes operating at $2 \mathrm{bits} / \mathrm{symbol}$ and $10 \mathrm{~dB}$ SNR per user. The results for $\beta=50$ and $N_{s}=1$ are given. We found that significant increase in code lengths is required in the presence of high amounts of MAI for 8-PPM as compared to 16-PPM and 32-PPM. Also, we observed that only a two-fold increase in the block length results in an 


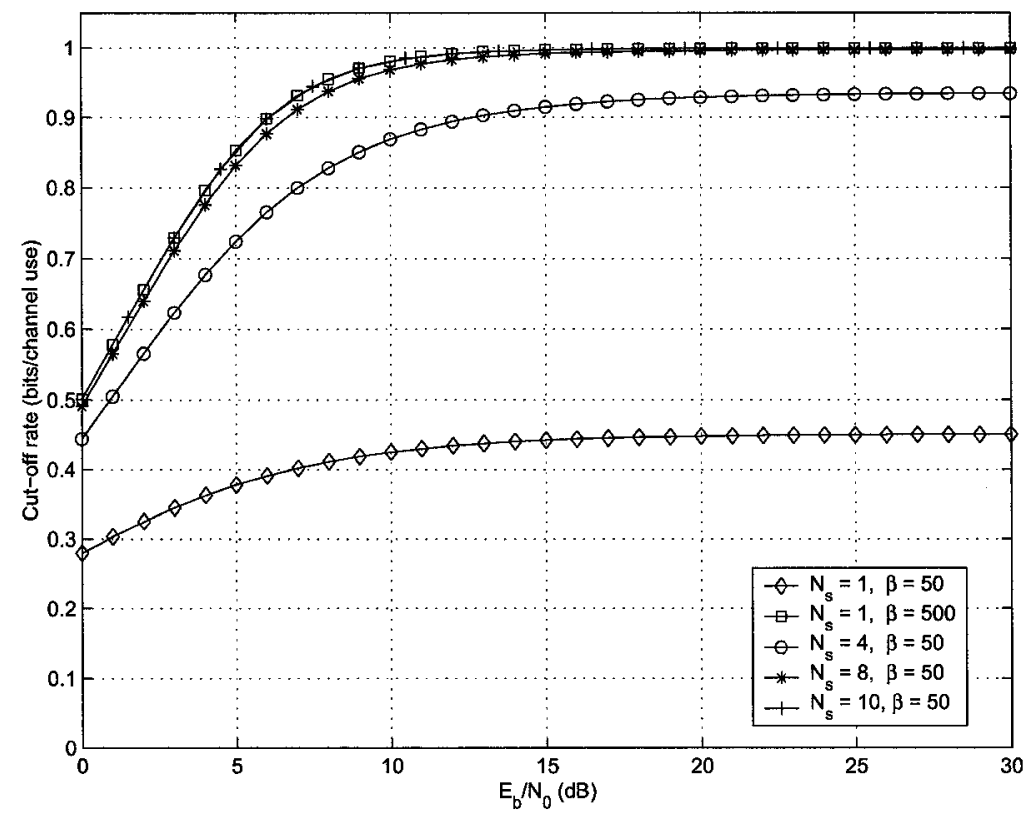

Figure B.3: Cutoff rates of TH 2-PPM multiuser UWB for different spreading and hopping factors for $\mathcal{N}_{u}=100$.

error rate reduction from $10^{-3}$ to $10^{-6}$ for all the M-ary schemes. A further investigation of the actual coding scheme for the system described in this letter can be undertaken. The work on capacity limit achievable by Reed-Solomon (R-S) M-ary PPM in AWGN presented in [99] can complement this effort.

\section{B.4 Conclusion}

This work evaluates the practical capacity, known as the cut-off rate, for TH-PPM adopted for UWB communication over multiple-access channels, without a need for numerical integration or Monte Carlo simulation. We have shown how the cutoff rate can be used in M-ary PPM UWB multiple-access communication systems for determining the system trade-offs. Moreover, cutoff rate evaluated in this letter can be a fast way of gaining insights into the multiuser capacity of TH-PPM UWB systems. 


\section{Appendix C}

\section{Derivations}

\section{C.1}

The incomplete gamma function is defined as [80]

$$
\Gamma_{\mathrm{L}}[a, b]=\int_{0}^{b} e^{-t} t^{a-1} d t
$$

Therefore,

$$
F_{\gamma}(\gamma, 1)=\Gamma_{\mathrm{L}}\left[1, \frac{\gamma}{\bar{\gamma}}\right]=\int_{0}^{\frac{\gamma}{\bar{\gamma}}} e^{-t} t^{1-1} d t=1-e^{-\frac{\gamma}{\bar{\gamma}}}
$$

\section{C.2}

The PDF of $\gamma$ can be derived given that $\gamma=\alpha^{2} E_{b} / N_{0}$, and PDF of $\alpha$ is known. We use the method of function of a RV.

$$
\alpha=\sqrt{\gamma \frac{N_{0}}{E_{b}}}, \Rightarrow \frac{d \gamma}{d \alpha}=2 \sqrt{\gamma E_{b} / N_{0}}
$$

Note that $\bar{\gamma}=\Omega E_{b} / N_{0}$, which implies $N_{0} / E_{b}=\Omega / \bar{\gamma}$. Then, $\alpha=\sqrt{\frac{\Omega \gamma}{\bar{\gamma}}}$ and $\frac{d \gamma}{d \alpha}=2 \sqrt{\frac{\gamma \bar{\gamma}}{\Omega}}$ is the Jacobian of the transformation. Hence,

$$
p_{\gamma}(\gamma)=(d \gamma / d \alpha)^{-1} p_{\alpha}(\sqrt{\Omega \gamma / \bar{\gamma}})=\frac{p_{\alpha}(\sqrt{\Omega \gamma / \bar{\gamma}})}{2 \sqrt{\frac{\gamma \bar{\gamma}}{\Omega}}}
$$


This concludes the derivation of (2.10)

\section{C.3}

We demonstrate how the integral in $P_{\text {mpsk }}\left(e \mid g^{*}\right)$ is evaluated to arrive at (3.5). The symbol error probability (SEP) for MPSK modulation conditioned on the fade samples can be expressed for GSC as

$$
P_{\mathrm{mpsk}}\left(e \mid \beta, g^{*}\right) \approx h \times \operatorname{erfc}\left(\sqrt{\beta \Lambda^{*} \sin ^{2}\left(\frac{\pi}{M}\right) \frac{E_{s}}{N_{0}}}\right) .
$$

We shall treat $(3.4)$ as $p\left(\beta, l_{\mathrm{o}}\right)=p_{1}\left(\beta, l_{\mathrm{o}}\right)+p_{2}\left(\beta, l_{\mathrm{o}}\right)+p_{3}\left(\beta, l_{\mathrm{o}}\right)$ where,

$$
p_{1}\left(\beta, l_{\mathrm{o}}\right)=\left(\begin{array}{c}
L \\
l_{\mathrm{o}}
\end{array}\right) \frac{\beta^{l_{\mathrm{o}}-1} e^{-\beta}}{\left(l_{\mathrm{o}}-1\right) !}, \quad p_{2}\left(\beta, l_{\mathrm{o}}\right)=\left(\begin{array}{c}
L \\
l_{\mathrm{o}}
\end{array}\right) \sum_{l=1}^{L-l_{\mathrm{o}}} \eta(l, n, N) e^{-\beta\left(1+l / l_{\mathrm{o}}\right)}
$$

and

$$
p_{3}\left(\beta, l_{\mathrm{o}}\right)=\left(\begin{array}{c}
L \\
l_{\mathrm{o}}
\end{array}\right) \sum_{l=1}^{L-l_{\mathrm{o}}} \eta\left(l, l_{\mathrm{o}}, L\right) \sum_{p=0}^{l_{\mathrm{o}}-2} \frac{1}{p !}\left(\frac{-l \beta}{l_{\mathrm{o}}}\right)^{p} e^{-\beta} .
$$

We express $P_{\mathrm{mpsk}}\left(e \mid g^{*}\right)=P_{1}+P_{2}+P_{3}$ and demonstrate how the integral in $P_{\mathrm{mpsk}}\left(e \mid g^{*}\right)=$ $\int_{0}^{\infty} P_{\mathrm{mpsk}}\left(e \mid \beta, g^{*}\right) p\left(\beta, l_{\mathrm{o}}\right) d \beta$ is evaluated to arrive at (3.5). First, let us define

$$
P_{1}=h\left(\begin{array}{c}
L \\
l_{\circ}
\end{array}\right) \int_{0}^{\infty} \frac{\beta^{l_{o}-1} e^{-\beta}}{\left(l_{\mathrm{o}}-1\right) !} \operatorname{erfc}\left(\sqrt{\beta \Lambda^{*} \lambda}\right) d \beta
$$

Let $\theta_{1}=\frac{\Lambda^{*} \lambda}{1+\Lambda^{*} \lambda}$ and $y=\beta\left(1+\Lambda^{*} \lambda\right)$, then we can write

$$
y \theta_{1}=\beta \Lambda^{*} \lambda
$$

With this representation we can express (C.7) as

$$
P_{1}=h\left(\begin{array}{c}
L \\
l_{\mathrm{o}}
\end{array}\right) \int_{0}^{\infty} \frac{y^{l_{\mathrm{o}}-1} e^{-y \theta_{1} /\left(\Lambda^{*} \lambda\right)}}{\left(l_{\mathrm{o}}-1\right) !\left(1+\Lambda^{*} \lambda\right)^{l_{\circ}}} \operatorname{erfc}\left(\sqrt{y \theta_{1}}\right) d y
$$


Next, we can show that $\frac{\theta_{1}}{\Lambda^{*} \lambda}=\left(1-\theta_{1}\right)$ and, thereafter, see that (C.9) is in the following form [74]:

$$
\frac{1}{2(W-1) !} \int_{0}^{\infty} \operatorname{erfc}(\sqrt{x y}) e^{-y(1-x)} y^{W-1} d y=\frac{1}{2^{2 W}} \sum_{j=1}^{W} C_{W-1}^{2 W-j-1}\left(\frac{2}{1+\sqrt{x}}\right)^{j} .
$$

From here $P_{1}$ is obtained in a straight forward manner which is the first part of (3.5).

Next, we have

$$
P_{2}=\int_{0}^{\infty} \operatorname{erfc}\left(\sqrt{\beta \Lambda^{*} \lambda}\right)\left(\begin{array}{c}
L \\
l_{\circ}
\end{array}\right) \sum_{l=1}^{L-l_{\mathrm{o}}} \eta\left(l, l_{\mathrm{o}}, L\right) e^{-\beta\left(1+l / l_{\mathrm{o}}\right)} d \beta .
$$

Let $\theta_{2}=\frac{\Lambda^{*} \lambda}{\left(l / l_{\mathrm{o}}+1+\Lambda^{*} \lambda\right)}$ and $\Gamma=\beta\left(l / l_{\mathrm{o}}+1+\Lambda^{*} \lambda\right)$ then (C.11) can be written in the following form

$$
P_{2}=\left(\begin{array}{c}
L \\
l_{\circ}
\end{array}\right) \sum_{l=1}^{L-l_{\mathrm{o}}} \eta\left(l, l_{\mathrm{o}}, L\right) \frac{1}{\left(l / l_{\mathrm{o}}+1+\Lambda^{*} \lambda\right)} \int_{0}^{\infty} \operatorname{erfc}\left(\sqrt{\theta_{2} \Gamma}\right) e^{-\Gamma \frac{\left(1+l / l_{\mathrm{o}}\right)}{1+l / l_{\mathrm{o}}+\Lambda^{*} \lambda}} d \Gamma .
$$

It can be shown that $\frac{1+l / l_{\circ}}{1+l / l_{\mathrm{o}}+\Lambda^{*} \lambda}=1-\theta_{2}$, therefore,

$$
P_{2}=\left(\begin{array}{c}
L \\
l_{\mathrm{o}}
\end{array}\right) \sum_{l=1}^{L-l_{\mathrm{o}}} \eta\left(l, l_{\circ}, L\right) \frac{1}{\left(l / l_{\circ}+1+\Lambda^{*} \lambda\right)} \int_{0}^{\infty} \operatorname{erfc}\left(\sqrt{\theta_{2} \Gamma}\right) e^{-\Gamma\left(1-\theta_{2}\right)} d \Gamma
$$

and hence,

$$
P_{2}=\left(\begin{array}{c}
L \\
l_{\mathrm{o}}
\end{array}\right) \sum_{l=1}^{L-l_{\mathrm{o}}} \eta\left(l, l_{\mathrm{o}}, L\right) \frac{1}{\left(l / l_{\mathrm{o}}+1+\Lambda^{*} \lambda\right)} \frac{1}{\left(1+\sqrt{\theta_{2}}\right)}
$$

and finally, by doing similar manipulations as performed above, $P_{3}$ can be expressed as

$$
P_{3}=\int_{0}^{\infty} \operatorname{erfc}\left(\sqrt{\theta_{1} \omega}\right)\left(\begin{array}{c}
L \\
l_{\circ}
\end{array}\right) \sum_{l=1}^{L-l_{\mathrm{o}}} \eta\left(l, l_{\mathrm{o}}, L\right) \sum_{p=0}^{l_{\mathrm{o}}-2}\left(-l / l_{\mathrm{o}}\right)^{p} \frac{1}{\left(1+\Lambda^{*} \lambda\right)^{p+1}} \frac{\omega^{p}}{p !} e^{-\omega\left(1-\theta_{1}\right)} d \omega .
$$

By interchanging the integral and the summation, the integration can be evaluated and the resulting expression simplifies to,

$$
P_{3}=\left(\begin{array}{c}
L \\
l_{\mathrm{o}}
\end{array}\right) \sum_{l=1}^{L-l_{\mathrm{o}}} \eta\left(l, l_{\mathrm{o}}, L\right) \sum_{p=0}^{l_{\mathrm{o}}-2}\left(-l / l_{\mathrm{o}}\right)^{p} \frac{1}{\left(1+\Lambda^{*} \lambda\right)^{p+1}} \frac{1}{2^{2 p+1}} \sum_{j=1}^{p+1}\left(\begin{array}{c}
2(p+1)-j-1 \\
p
\end{array}\right)\left(\frac{2}{1+\sqrt{\theta_{1}}}\right)^{j} .
$$


Combining $P_{1}, P_{2}$, and $P_{3}$ we obtain the desired expression for $P_{\mathrm{mpsk}}\left(e \mid g^{*}\right)$.

\section{4}

For MRC, the normalizing factor is

$$
v_{\mathrm{MRC}}=\int_{\gamma_{t h}}^{\infty}\left(\frac{m}{\bar{\gamma}}\right)^{L m} \frac{\gamma^{L m-1}}{\Gamma[L m]} \exp \left(-\frac{m \gamma}{\bar{\gamma}}\right) d \gamma
$$

With the definition of the incomplete gamma function the expression in (C.17) can be expressed as

$$
v_{\mathrm{MRC}}=\frac{\Gamma\left[L m, \frac{m \gamma_{t h}}{\bar{\gamma}}\right]}{\Gamma[L m]} .
$$

For Rayleigh fading $(m=1)$ and no diversity antennas at relay (i.e., $L=1$ ),

$$
v=\Gamma\left[L m, \frac{m \gamma_{t h}}{\bar{\gamma}}\right]=\Gamma\left[1, \gamma_{t h} / \bar{\gamma}\right]=\exp \left(-\gamma_{t h} / \bar{\gamma}\right)
$$

For Nakagami SC, the normalizing factor is

$$
\begin{aligned}
v_{\mathrm{SC}} & =\int_{\gamma_{t h}}^{\infty} \frac{L}{(m-1) !} \sum_{l=0}^{L-1}(-1)^{l}\left(\begin{array}{c}
L-1 \\
l
\end{array}\right) \sum_{k=0}^{l(m-1)} b_{k}^{l}\left(\frac{m}{\bar{\gamma}}\right)^{m+k} \gamma^{m+k-1} \exp \left(-(l+1) \frac{m \gamma}{\bar{\gamma}}\right) d \gamma \\
& =\frac{L}{(m-1) !} \sum_{l=0}^{L-1}(-1)^{l}\left(\begin{array}{c}
L-1 \\
l
\end{array}\right) \sum_{k=0}^{l(m-1)} b_{k}^{l}\left(\frac{1}{1+l}\right)^{k+m} \Gamma\left[k+m,(1+l) \frac{m \gamma_{t h}}{\bar{\gamma}}\right] . \quad \text { (C.20) }
\end{aligned}
$$

\section{C.5}

Computing $\pi_{i, j}$ for the selected scenarios.

One cooperative relay: with $m_{1}=1$ and $m_{2}=2$.

$$
\begin{gathered}
\pi_{i, j}=\frac{1}{\left(m_{i}-j\right) !\left(-\frac{\bar{\gamma}_{i}}{m_{i}}\right)^{m_{i}-j}}\left\{\frac{\partial^{m_{i}-j}}{\partial \omega^{m_{i}-j}}\left(1-\frac{\bar{\gamma}_{i}}{m_{i}} w\right)^{m_{i}} \psi_{\gamma}(\omega)\right\}_{\omega=\frac{m_{i}}{\bar{\gamma}_{i}}} . \\
\pi_{1,1}=\left.\frac{1}{(1-1) !\left(\frac{-\bar{\gamma}_{1}}{m_{1}}\right)^{1-1}}\left(1-\omega \frac{\bar{\gamma}_{1}}{1}\right)^{1} \frac{1}{\left(1-\omega \frac{\bar{\gamma}_{1}}{1}\right)\left(1-\omega \frac{\bar{\gamma}_{2}}{2}\right)^{2}}\right|_{\omega=\frac{1}{\bar{\gamma}_{1}}}=\left(1-\frac{\bar{\gamma}_{2}}{2 \bar{\gamma}_{1}}\right)^{2},
\end{gathered}
$$

next $\pi_{2,1}$ 


$$
\begin{aligned}
\pi_{2,1} & =\left.\frac{1}{(2-1) !\left(\frac{-\bar{\gamma}_{2}}{2}\right)^{2-1}} \frac{\partial}{\partial \omega}\left(1-\omega \frac{\bar{\gamma}_{2}}{2}\right)^{2} \frac{1}{\left(1-\omega \frac{\bar{\gamma}_{1}}{1}\right)\left(1-\omega \frac{\bar{\gamma}_{2}}{2}\right)^{2}}\right|_{\omega=\frac{2}{\bar{\gamma}_{2}}} \\
& =\left.\frac{1}{(2-1) !\left(\frac{-\bar{\gamma}_{2}}{2}\right)^{2-1}} \frac{\partial}{\partial \omega} \frac{1}{\left(1-\omega \bar{\gamma}_{1}\right)}\right|_{\omega=\frac{2}{\bar{\gamma}_{2}}}=\frac{-2 \bar{\gamma}_{1}}{\bar{\gamma}_{2}} \frac{1}{\left(1-\frac{2 \bar{\gamma}_{1}}{\bar{\gamma}_{2}}\right)^{2}}
\end{aligned}
$$

then $\pi_{2,2}$

$$
\begin{aligned}
\pi_{2,2} & \left.=\frac{1}{(2-2) !\left(\frac{\overline{\bar{\gamma}_{2}}}{2}\right)^{2-2}} \frac{\partial^{2-2}}{\partial \omega^{2-2}}\left(1-\omega \frac{\bar{\gamma}_{2}}{2}\right)^{2} \frac{1}{\left(1-\omega \frac{\bar{\gamma}_{1}}{1}\right)\left(1-\omega \overline{\bar{\gamma}_{2}}\right.}\right)\left.^{2}\right|_{\omega=\frac{2}{\bar{\gamma}_{2}}} \\
& =\left.\frac{1}{\left(1-\omega \frac{\bar{\gamma}_{1}}{1}\right)}\right|_{\omega=\frac{2}{\bar{\gamma}_{2}}}=\frac{1}{\left(1-\frac{2 \bar{\gamma}_{1}}{\bar{\gamma}_{2}}\right)} .
\end{aligned}
$$

Two cooperative relays: with $m_{1}=1, m_{2}=2$, and $m_{3}=2$, the CF for this scenario is

$$
\psi(\omega)=\frac{1}{\left(1-\omega \bar{\gamma}_{1} / 1\right)\left(1-\omega \bar{\gamma}_{2} / 2\right)^{2}\left(1-\omega \bar{\gamma}_{3} / 2\right)^{2}}
$$

Therefore

$$
\pi_{1,1}=\left.\frac{1}{(1-1) !}\left(-\bar{\gamma}_{1} / 1\right)^{1-1} \frac{1}{\left(1-\omega \bar{\gamma}_{2} / 2\right)^{2}\left(1-\omega \bar{\gamma}_{3} / 2\right)^{2}}\right|_{\omega=\frac{1}{\bar{\gamma}_{1}}}=\frac{1}{\left(1-\frac{\bar{\gamma}_{2}}{2 \bar{\gamma}_{1}}\right)^{2}\left(1-\frac{\bar{\gamma}_{3}}{2 \bar{\gamma}_{1}}\right)^{2}}
$$

and,

$$
\begin{aligned}
\pi_{2,1} & =\left.\frac{1}{(2-1) !\left(\frac{-\overline{\gamma_{2}}}{2}\right)^{2-1}} \frac{\partial}{\partial \omega}\left(1-\omega \frac{\bar{\gamma}_{2}}{2}\right)^{2} \frac{1}{\left(1-\omega \frac{\bar{\gamma}_{1}}{1}\right)\left(1-\omega \frac{\bar{\gamma}_{2}}{2}\right)^{2}\left(1-\omega \frac{\bar{\gamma}_{3}}{2}\right)^{2}}\right|_{\omega=\frac{2}{\bar{\gamma}_{2}}} \\
& =\left.\left(\frac{-2}{\bar{\gamma}_{2}}\right) \frac{\partial}{\partial \omega} \frac{1}{\left(1-\omega \frac{\bar{\gamma}_{1}}{1}\right)\left(1-\omega \frac{\bar{\gamma}_{3}}{2}\right)^{2}}\right|_{\omega=\frac{2}{\bar{\gamma}_{2}}}
\end{aligned}
$$

employing the product rule for differentiation, $\frac{d}{d x} u v=u \frac{d v}{d x}+v \frac{d u}{d x}$, we have,

$$
=\frac{-2}{\bar{\gamma}_{2}}\left\{\frac{\bar{\gamma}_{3}}{\left(1-\omega \bar{\gamma}_{1}\right)\left(1-\omega \bar{\gamma}_{3} / 2\right)^{3}}+\frac{\bar{\gamma}_{1}}{\left(1-\omega \bar{\gamma}_{1}\right)^{2}\left(1-\omega \bar{\gamma}_{3} / 2\right)^{2}}\right\}_{\omega=\frac{2}{\bar{\gamma}_{2}}} .
$$

Upon doing the substitution $\pi_{2,1}$ of (5.12) appears. In similar manner, $\pi_{2,2}, \pi_{3,1}$, and $\pi_{3,2}$ can be found. 


\section{Bibliography}

[1] H. Yanikomeroglu, "Tutorial: Infrastructure-based wireless multihop/relay/mesh networks," IEEE Globecom 2004, Dec. 2004.

[2] IEEE Std 802.16-2004, "IEEE standard for local and metropolitan area networks Part 16: Air interface for fixed broadband wireless acesss systems," 2004.

[3] IST WINNER D3.2, "Description of deployment concepts for future radio scenarios integrating different relaying technologies in a cellular infrastructure including definition, assessment and performance comparison of RAN protocols for relay based systems," https://www.ist-winner.org/DeliverableDocuments/D3-2.pdf (available on-line June 2005), Feb. 2005.

[4] C. Eklund, R. Marks, K. Stanwood, and S. Wang, "IEEE standard 802.12: A technical overview of the WirelessMan ${ }^{\mathrm{TM}}$ air interface for broadband wireless acesss," IEEE Communications Magazine, pp. 98-107, June 2002.

[5] A. Maitra, Wireless spectrum management: Policies, Practices, and Conditioning Factors, New York: McGraw-Hill, 2004.

[6] H. Poor and G. Wornell, Wireless Communications, Prentice-Hall, Inc, 1998.

[7] G. J. Foschini and M. J. Gans, "On limits of wireless communications in fading environment when using multiple antennas," Wireless Personal Communication, vol. 6, pp. 311-335, Mar. 1998. 
[8] L. Zheng and D. N. C. Tse, "Diversity and multiplexing: A fundamental tradeoff in multiple antenna channels," IEEE Transactions Information Theory, 49(5), pp. 10731096, May 2003.

[9] G. Bauch and J. Hagenauer, "Smart versus dumb antennas-capacities and FEC performance," IEEE Communication Letters, vol. 6, no. 2, pp. 55-57, Feb. 2002.

[10] S. Alamouti, "A simple transmit diversity technique for wireless communication," IEEE Journal on Selected Areas in Communications, 16(8), pp. 1451-1458, Oct. 1998.

[11] P. Viswanth, D. Tse and R. Laroia, "Opportunistic beamforming using dumb antennas," IEEE Transactions on Information Theory, pp. 1277-1294, June 2002.

[12] E. Telatar, "Capacity of multi-antenna Gaussian channels," European Transactions on Telecommunication, vol. 6, pp. 585-595, Nov. 1999.

[13] Z. J. Haas and C.-P. Li, "The multiply-detected macrodiversity scheme for wireless cellular systems," IEEE Transactions on Vehicular Technology, 47(2), pp. 506-530, May 1998.

[14] J.-G. Chen, N. Ansari, and Z. Siveski, "Performance analysis and realization of decision fusion for macroscopic diversity in cellular wireless systems," IEEE GLOBECOM, pp. 424-428, Nov. 1997.

[15] A. Salmasi and K. Gilhousen, "On the system design aspects of code division multiple access (CDMA) applied to digital cellular and personal communication networks," IEEE Vehicular Technology Conference, pp. 57-62, 1991.

[16] H. Yanikomeroglu and E. S. Sousa, "CDMA distributed antenna system for indoor wireless communications," International Conference on Universal Personal Communications, pp. 990-994, Oct. 1993. 
[17] H. Yanikomeroglu and E. S. Sousa, "CDMA sectorized distributed antenna system," IEEE International Symposium on Spread Spectrum Techniques and Applications, pp. 792-797, Sep. 1998.

[18] L. Dai, S.-D. Zhou, and Y. Yao, "Capacity with MRC-based macrodiversity in CDMA distributed antenna systems," IEEE GLOBECOM, vol. 1, pp. 987-991, Nov. 2002.

[19] J.-S. Wu, J. Wu, and H.-W. Tsao, "A radio-over-fiber network for microcellular system application," IEEE Transactions on Vehicular Technology, (47)1, pp. 84-94, Feb. 1998.

[20] J. Winters, "Optimum combining in digital mobile radio with cochannel interference," IEEE Journal on Selected Areas in Communications, no. 4, pp. 528-539, July 1984.

[21] J. Winters, "The diversity gain of transmit diversity in wireless systems with Rayleigh fading," IEEE International Conference on Communications, vol. 2, pp. 1121-1125, May 1994.

[22] J. Winters, J. Salz, and R. Gitlin, "The impact of antenna diversity on the capacity of wireless communication systems," IEEE Transactions on Communications, vol. 42, pp. 1740-1751, Mar. 1994.

[23] M. Simon and M. Alouini, Digital Communication over Fading Channels, John Wiley, 2005.

[24] N. Kong and L. B. Milstein, "Combined average SNR of a generalized diversity selection combining scheme," IEEE International Conference on Communications, pp. 1556-1560, June 1998.

[25] M. Alouini and M. Simon, "Performance of coherent recievers with hybrid SC/MRC over Nakagami-m fading channels," IEEE Transactions on Vehicular Technology, 48(4), pp. 1155-1164, July 1999. 
[26] M-S. Alouini and M. K. Simon, "An MGF-based performance analysis of generalized selection combining over Rayleigh fading channels," IEEE Transactions on Communications, 48(3), pp. 401-415, Mar. 2000.

[27] M. Z. Win and J. H. Winters, "Virtual branch analysis of symbol error probability for hybrid selection/maximal-ratio combining in Rayleigh fading," IEEE Transactions on Communications, 11(49), pp. 1926-1934, Nov. 2001.

[28] A. Adinoyi and S. Al-Semari, "Analysis of coherent MPSK schemes with generalized selection diversity in Rayleigh fading," European Transactions on Telecommunications, pp. 101-105, April 2005.

[29] J. G. Proakis, Digital Communications, McGraw Hill, 1995.

[30] M. Win and R. Scholtz, "Characterization of ultra-wide badnwidth wireless indoor channels: A communication-theoretic view," IEEE Journal on Selected Areas in Communications, pp. 1613-1624, Dec. 2002.

[31] R. Price and P. Green, "A communication technique for multipath channels," Proceeding of IRE, vol. 46, pp. 550-570, Mar. 1958.

[32] Theodore S. Rappaport, Wireless Communications, Prentice Hall, 1996.

[33] P. Herhold, "Cooperative Relaying - Protocols and Performance," Shaker Verlag, Aachen 2005.

[34] H. Yanikomeroglu, "Fixed and mobile relaying technologies for cellular networks", Second Workshop on Applications and Services in Wireless Networks, pp. 75-81, July 2002.

[35] A. Florea, On the Efficiency of Using Multiple Hops in Fixed Relay Based Wireless Networks, Master's Thesis at Carleton University (Supervised by H. Yanikomeroglu), 2004. 
[36] R. Pabst, B. Walke, D. Schultz, P. Herhold, H. Yanikomeroglu, S. Mukherjee, H. Viswanathan, M. Lott, W. Zirwas, M. Dohler, H. Aghvami, D. Falconer, and G. Fettweis, "Relay-based deployment concepts for wireless and mobile broadband radio," IEEE Communication Magazine, 42(9), pp. 80-89, Sep. 2004.

[37] G. L. Stuber, Principles of Mobile Communications, Boston: Kluwer Academic, 1996.

[38] J. Laneman, D. Tse, and G. Wornell, "Cooperative diversity in wireless networks: efficient protocols and outage behavior," IEEE Transactions on Information Theory, 50(11), pp. 3062-3080, Dec. 2004.

[39] A. Sendonaris, E. Erkip, and B. Aazhang "User cooperation diversity - Part I: system description," IEEE Transactions on Communications, 51(11), pp. 1927-1938, Nov. 2003.

[40] J. Laneman and G. Wornell, "Distributed space-time-coded protocols for exploiting cooperative diversity in wireless networks," IEEE Transactions on Information Theory, 49(10), pp. 2415-2425, Oct. 2003.

[41] J. Boyer, H. Yanikomeroglu, and D. Falconer, "Multihop diversity in wireless relaying channels," IEEE Transactions on Communications, 52(10), pp. 1820-1830, Oct. 2004.

[42] M. Hasna and M. Alouini, "End-to-end performance of transmission systems with relays over Rayleigh-fading channels," IEEE Transactions on Wireless Communications, 2(6), pp. 1126-1131, Nov. 2003.

[43] B. Rankov and A. Wittneben, "On the capacity of relay-assisted Wireless MIMO channels," Fifth IEEE Workshop on Signal Processing Advances in Wireless Communications, July 2004 .

[44] A. Wittneben and B. Rankov, "Impact of cooperative relays on capacity of rank deficient MIMO channels," IST Summit on Mobile and Wireless Communications, pp. 421-425, June 2003. 
[45] Y. Hua, Y. Mei, and Y. Chang, "Parallel wireless mobile relays with space-time modulations," IEEE Workshop on Statistical Signal Processing, pp. 375-378, Oct. 2003.

[46] N. Khajehnour and A. Sayed, "Optimal multi-relay strategy for imperfect channel information in sensors networks," European Signal Processing Conference, Sep. 2005.

[47] P. Larsson and H. Rong, "Large-scale cooperative relaying network with optimal coherent combining under aggregate relay power constraints," World Wireless Research Forum (WWRF) Meeting no. 8, Sep. 2005.

[48] P. Anghel, G. Leus, and M. Kaveh, "Multi-user space-time coding in cooperative networks," IEEE International Conference on Acoustics, Speech, and Signal Processing, pp. IV-73 - IV-76, Apr. 2003.

[49] D. Chen and J. Laneman "Noncoherent demodulationn for cooperative diversity in wireless systems," IEEE GLOBECOM, vol. 1, pp. 31-35, Dec. 2004.

[50] M. Yuksel and E. Erkip, "Diversity in relaying protocols with amplify and forward," IEEE GLOBECOM, pp. 2025-2029, 2003.

[51] T. Hunter and A. Nosratinia, "Cooperation diversity through coding," IEEE International Symposium on Information Theory, pp. 220, 2002.

[52] M. Dohler and H. Aghvami, "Distributed PHY-layer mesh network," IEEE Personal, Indoor and Mobile Communication Conference, vol. 3, pp. 2543-2547, Sep. 2003.

[53] S. Barbarossa and G. Scutari, "Cooperative diversity through virtual arrays in multihop network," IEEE International Conference on Acoustics, Speech, and Signal Processing 2003, vol. 4, pp. 209-2012, Apr. 2003.

[54] E. Meulen "Three-terminal communication channels," Advanced Applied Probability, vol. 3, pp. $120-154,1971$. 
[55] T. Cover and A. El-Gamal, "Capacity theorems for the relay channel," IEEE Transactions on Information Theory, 25(5), pp. 572-584, Sep. 1979.

[56] M. Janani, A. Hedayat, T. Hunter, and A. Nosratinia, "Coded cooperation in wireless communications: Space-time transmission and iterative decoding," submitted to IEEE Transactions on Signal Processing, 2003.

[57] A. Stefanov and E. Erkip "Cooperative coding for wireless networks," IEEE Transactions on Communications, 52(9), pp. 1470-1476, Sep. 2004.

[58] P. Herhold, E. Zimmermann, and G. Fettweis, "A simple cooperation extension to wireless relaying," International Zurich Seminar on Communications, Feb. 2004.

[59] W. Siriwongpairat, T. Himsoon, W. Su, and K. Liu, "Optimum threshold-selection relaying for decode-and-forward cooperation protocol," IEEE Wireless Communications and Networking Conference, Apr. 2006 (Conference CDROM).

[60] R. Ohmoto, H. Ohtsuka, and H. Ichikawa, "Fiber-optic microcell radio system with a spectrum delivery scheme," IEEE Journal on Selected Areas on Communications, pp. 1108-1117, Sep. 1993.

[61] H. Hu, H. Yanikomeroglu, D. Falconer, and S. Periyalwar, "Range extension without capacity penalty in cellular networks with digital fixed relays," IEEE GLOBECOM, 2004 .

[62] V. Morgenshtern and H. Bölcskei "On the value of cooperation in interference relay networks," Allerton Conference, Dec. 2005.

[63] O. Munoz, A. Agustin, and J. Vidal, "Cellular capacity gains of cooperative MIMO transmission in the downlink," IEEE International Zurich Seminar on Communications, Feb. 2004. 
[64] A. Adinoyi, H. Yanikomeroglu, and S. Loyka, "Hybrid macro and generalized selection combining microdiversity in lognormal shadowed Rayleigh fading channels," IEEE International Conference on Communications, June 2004.

[65] A. Adinoyi and H. Yanikomeroglu, "On the performance macro/microdiversity in the reverse-link wireless networks," IEEE Wireless Communications and Networking Conference, vol. 2, pp. 973-977, Mar. 2005.

[66] W.-P. Yung, "Probability of bit error for MPSK modulation with diversity reception in Rayleigh fading and log-normal shadowing channel," IEEE Transactions on Communications, 38(7), pp. 933-937, July 1990.

[67] R. Mallik, M. Win, M. Chiani, and A. Zanella, "Bit-error probability for optimum combining of binary signals in the presence of interference and noise," IEEE Transactions on Wireless Communications, 3(2), pp. 395-407 Mar. 2004.

[68] W. Roh and A. Paulraj, "Outage performance of the distributed antenna systems in a composite fading channel," IEEE Vehicular Technology Conference, pp. 1520-1524, Fall 2002.

[69] A. A. Abu-Dayya and N. C. Beaulieu, "Micro- and macrodiversity MDPSK on shadowed frequency-selective channels," IEEE Transactions on Communications, 43(8), pp. 23342343, Aug. 1995.

[70] A. Conti, M. Z. Win, M. Chiani, and J. Winters, "Bit error outage for diversity reception in shadowing environment," IEEE Communication Letters, 7(1), pp. 15-17, 2003.

[71] L. Wang, G. Stuber, and C. Lea, "Effect of Rician fading and branch correlation on a local-mean-based macrodiversity cellular system", IEEE Transactions on Vehicular Technology, 48(2), pp. 429-436, Mar. 1999. 
[72] J. Zhang and V. Aalo, "Effect of Macrodiversity on average-error probabilities in a Rician fading channel with correlated lognormal shadowing", IEEE Transactions on Communications, 49(1), pp. 472-482, Jan. 2001

[73] S. Hanly, "Capacity and power control in spread spectrum macrodiversity radio networks," IEEE Transactions on Communications, 44(2), pp. 247-256, Feb. 1996.

[74] S. H. Jamali and T. Le-Ngoc, Coded-Modulation Techniques for Fading Channels, Kluwer Academic Publishing, 1994.

[75] R. Braun and U. Dersch, "A physical mobile radio channel model," IEEE Vehicular Transactions, 40(2), pp. 472-482, May 1991.

[76] A. Wittneben, "Basestation modulation diversity for digital SIMULCAST," IEEE Vehicular Technology Conference, pp. 848-853, 1991.

[77] A. Wittneben, "A new bandwidth efficient transmit antenna modulation diversity scheme for linear digital modulation," IEEE International Conference on Communications, pp. 1630-1634, 1993.

[78] Y. Tu and G. Pottie, "Coherent cooperative transmission from multiple adjacent antennas to a distant stationary antenna through AWGN channels," IEEE Vehicular Technology Conference, pp. 130-134, 2002.

[79] A. Papoulis, Probability, Random Variables, and Stochastic Processes, New York: McGraw-Hill, 1991.

[80] I. Gradshteyn and I. Ryzhik, Table of Integrals, Series, and Products, San Diego, CA: Academic Press, 1994.

[81] S. Wolfram, Mathematica: A System for Doing Mathematics by Computer, Addison Wesley Publishing, Inc, 1988. 
[82] G. Fedele, "Error probability for diversity detection of binary signals over Nakagami fading channels," IEEE International Conference on Personal Indoor Radio and Mobile Communications, pp. 609-611, 1994.

[83] E. Al-Hussaini and A. Al-Bassiouni, "Performance of MRC diversity systems for the detection of signals in Nakagami fading," IEEE Transactions on Communications, vol. 33, pp. 1315-1319, Feb. 1985.

[84] U. Charash, "Reception through Nakagmi fading multipath channels with random delays," IEEE Transactions on Communication, 27, pp. 657-670, Apr. 1979.

[85] W. Yuen, R. Yates, and C. Sung, "Performance evaluation of highway mobile infostation networks," IEEE GLOBECOM, pp. 934-939, Dec. 2003.

[86] S. Verdu, Multiuser Detection, Cambridge University Press, 1998.

[87] H. Van Trees, Optimum Array Processing, Wiley, 2002.

[88] J. Laneman, D. Tse, and G. Wornell, "Cooperative diversity in wireless networks: efficient protocols and outage behavior," IEEE Transactions on Information Theory, 50(11), pp. 3062-3080, Dec. 2004.

[89] A. Adinoyi and H. Yanikomeroglu, "Cooperative relaying in multi-antenna fixed relay networks," accepted to IEEE Transactions on Wireless Communications, May 2006.

[90] B. Wang, J. Zhang, and A. Madsen, "On the capacity of MIMO relay channels," IEEE Transactions on Information Theory, 51(1), pp. 29-43, Jan. 2005.

[91] A. V. Oppenheim and R. W Schafer, Discrete-Time Signal Processing, Prentice Hall, 1989.

[92] M. Dohler and H. Aghvami, "Information outage probability of distributed STBCs over Nakagami fading channels," IEEE Communication Letters, 8(7), pp. 437-439, July 2004. 
[93] M. Z. Win and R. A. Scholtz, "Ultra-wide bandwidth time-hopping spread-spectrum impulse radio for wireless multiple-access communications," IEEE Transactions on Communications, vol. 48, pp. 679-691, Apr. 2002.

[94] W. K. Ahmed and P. J. McLane, "Random coding error exponents for flat fading channels with realistic channel estimation," IEEE Journal on Selected Areas in Communications, JSAC-18, pp. 369-379, Mar. 2000.

[95] J. Davidson and I. Kalet, "Bhattacharyya bound, cutoff rate, and constellation design for the companding channel," IEEE Transactions on Communications, vol. 45, no. 7, pp. 770-787, July 1997.

[96] E. Biglieri, J. Proakis, and S. Shamai, "Fading channels: Information-theoretic and communications aspects," IEEE Transactions on Information Theory, vol. 44, pp. 26192692, Oct. 1998.

[97] B. Hu and N. Beaulieu, "Exact bit error rate analysis of TH-PPM UWB systems in the presence of multiple-access interference," IEEE Communication Letters, vol. 7, no. 12, pp. 572-574, Dec. 2003.

[98] M. Abdel-Hafez, F. Alagoz, and M. Hamalainen, "Performance of M-ary PPM UWB radio in fading channels," Journal of Communication Networks, pp. 365-373, Dec. 2003.

[99] S. Dolinar, D. Divsalar, J. Hamkins, and F. Pollara, "Capacity of pulse-position modulation (PPM) on Gaussian and Webb channels," JPL/NASA TMO progress report 42-142 (JPL), Aug. 2000

(available online at http://tmo.jpl.nasa.gov/tmo/progress_report/tmo.html). 